\title{
SouthâMPIITS OF
}

\section{University of Southampton Research Repository}

Copyright $(\subset$ and Moral Rights for this thesis and, where applicable, any accompanying data are retained by the author and/or other copyright owners. A copy can be downloaded for personal non-commercial research or study, without prior permission or charge. This thesis and the accompanying data cannot be reproduced or quoted extensively from without first obtaining permission in writing from the copyright holder/s. The content of the thesis and accompanying research data (where applicable) must not be changed in any way or sold commercially in any format or medium without the formal permission of the copyright holder/s.

When referring to this thesis and any accompanying data, full bibliographic details must be given, e.g.

Thesis: Author (Year of Submission) "Full thesis title", University of Southampton, name of the University Faculty or School or Department, PhD Thesis, pagination.

Data: Author (Year) Title. URI [dataset] 
THE STRUCTURE OF TURBULENT SHEAR FLOW

by

Philip John Morris, B.Sc。(Hons。)Eng。, M.Sc.

A Thesis submitted for the degree of Doctor of Philosophy in the Faculty of Engineering。

Department of Aeronautics and Astronautics University of Southampton

August, 1971. 
o. 17 line 1 ' $(1.30)^{\prime}$ becomes ' $(1.20)$ '

p. 17 line 3 ' $^{\prime}(1.31)^{\prime}$ becones ' (1.21)'

p. 28 line 18 insert $(2.21)^{\prime}$ after equation

p. 82 line $10{ }^{\prime}(v)_{\delta_{0}}$ becomes ${ }^{\prime}(v)_{S_{0}}$ '

p. 82 line 11 ' $\mathrm{A}_{\mathrm{z}}^{\prime}$ becomes 'A'

p. 85 Iine 16 insert 'number' after Reynolds

p. 87 line 19 delete 'this manner'. Insert 'Schubauer and Skramstads manner

p. 90 Line $8 \quad ' R_{\theta}=\frac{U_{\alpha} \theta}{v}$ becomes ${ }^{\prime} R_{\theta}=\frac{U_{d}^{\theta}}{v} '$

p.105 line $13 \quad{ }^{\prime} \ell=\left(\frac{U_{x}}{U_{d}}\right)^{\frac{1}{2}}$ becones ${ }^{\prime} \quad \ell=\left(\frac{u x}{U_{d}}\right)^{\frac{1}{2}}$

p.130 line $7,+\frac{1}{k_{1}} \int_{-\infty}^{\infty} \int_{-\infty}^{\infty} k_{1} \hat{\psi}\left(k_{1}, w\right) \ldots$ becomes $+\frac{1}{k_{1}} \int_{-\infty}^{\infty} \int_{-\infty}^{\infty} k_{1} \hat{\psi}\left(k_{1}, w\right) \ldots$,

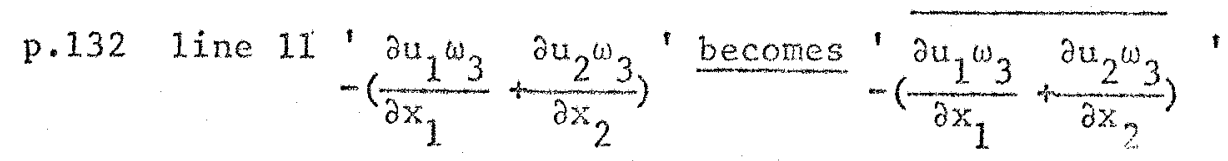

p. 150. line 1 delete sentence begiming 'The experimental results..."

insert The theoretical results are ploted such that

their maximum value coincides with the maximum experimental value'.

p.169 line 6 'equation $(7.30)^{\prime}$ becomes 'equation (7.31)' 


\section{BACULTY OF ENGTNESRTNG}

AERONAUTICS AND ASTRONAUTICS

\section{Doctor of Philosophy}

THE GTRUCTURE OF TURBULENT SHEAR FLOW

by Philip John Morris.

A theoretical investigation is made of the mixing layer between two streans. The work is divided into four sections. The first involves the solution of the mean problem of laminar and turbulent mixing. The equations of motion are written in terms of a similarity variable. An eddy viscosity hypothesis is made to describe the shear stresses. The similarity equations for both Iaminar and turbulent problens are solved numerically by an iterative schene.

The second section examines the stability of the mixing layer. The ortw-Somexteld equation of hydrodynamic stability is solved numerically, Both cases of spatial and temporal amplification are examined. Whe shear layer is shown to be unstable to both spatially and temporally growing disturbances at all Reynolds numbers. A correction is made for the aivergence of the mean fow and leads to a talue of critical Reynolds number of 12.3 . The third section presents a model for the turbulent mixing Iayer. A set of partial differential equations describing the Plow are obtained. A Fourier transform technique is employed 
to reduce this set to an ordinary differential equation for the fluctuating flow fold the homogeneous form or this equation is solved muerically. The resulting predictions of fluctuating velocity, pressure and their correlations are compared with measured values. The agreement is good in certain cases and this serves as a guide to components of the flow governed by non-linear processes.

The final section examines the non-linear growth of the mixing layex through transition from laminar to turbulent flow. A set of integral momentum and energy equations are woitten in terms of a number of shape parameters of mean and fuctuating fiow. The amplitude of disturbances is show to grow rapidly and reach a Iimiting value. A sudden growth of the mixing layer thickness through transition is also predicted. Comparison is made with experinental results. 
The work was performed at the University of Southanpton in the Department of Aeronautics and Astronautics and the Institute of Sound and Vibration Research.

The axthor wishes to thank the Science Research Council and the Ministry of Technology for their financial support in this project.

The author gratefully acknowledges the help and encouragement of Professor G.MLilley in his supervision of this work.

The author also wishes to thank members of the Computing Service at Southampton University and the Atlas Computing Laboratory, Chilton for their help. Thanks are also due to the many members of staff and friends at Southampton for their helpful aiscussions and ideas. The author is also grateful to Mrs. Wo Tudor Pole for typing this thesis so quickly.

Pinaly the author whes to thank his wife for her patience during many hours as a computer widow and her extra financial assistance. 
1. Introduction

2. Mathematical Derivation. 19

2.1 Governing Differentiol Equations.

2.2 Derivation of the squations for the Stability of

the Viscous Shear Layer。

2.3 The Integral Equations for the Flow

3. The Solution of the Mear Ruations for the Mixing of Tho Streams.

3.1. Introduction。

3.2 The Laminar Mixing of Two Parallel Streams.

3.3 The Thxoblent Mixing of Two Paxallel Streams.

3. 4 Solution of the Similarity Equations for Laminar and Turbulent Mixing Layers.

3.5 Computation Results。

4. Reviev of Methods of Solution of the OrrSommereld Equation.

4.1 Analytical Methodso

4.2 Numerical Mechods. 74

4.3 Spatial and Temporal Amplification. 84

5. The Stability of the Mixing Layer Between Two Streams. 89

5.1. Wumerical Method of Solution.

5.2 Numerical Calculation Procedure.

5.3 Review of Studies of the Stability of Free Shear Layers.

5.4 The Stability of Non-Paraliel Flows.

5.5 Results and Discussion.

5.6 Conclusions.

6. A Model of the Turobulent Mixing Layex Between

Two Streams:

6.I Governing Differential Equations.

6.2 Numerical Calculation Procedure for the Solution of the Homogeneous Equation for Turbulent Shear Flow。 
6.3 Discussion of Results.

6.4 Summery of Results of Iinear Aralysis.

7 . Finite Amplitude Lrects on the Development of the Mixing Layer.

7.I Introduction.

7.2 Coveming Differential and Integral Equations.

7.3 Shape Assumptions for the Mean Flow.

7.4 Shape As sumptions for the Fructuations.

7.5 Calculation of the Integral Functions.

7.6 Results and Discussion.

7.7 Conclusions.

8. Conclusions.

Appendix I

Discussion of the mirre Boundary Condition for the Mixing of Two Parallel Streams.

Appendix II. The Use of Orthogonalisation Procedure in Numerical Solution of Boundary-Value problems.

References.

pebles I and II

pigures. 


\section{CHAPTER 1}

\section{Introduction}

The notion of a fluid may be conveniently classified under three separate headings: steady laminar flow, unsteady laminar flow and turbulent flow. These classes of motjon are best described by reference to a series of identical experiments. If a property of the fluid, such as velocity or pressure, is measured in all the experiments then, in the case of steady Ieminar flow, it will not vary with time or from experiment to experiment. For the second type of motion, unsteady laminar flow, we would find that, although the measured property is a function of time, it will not vary from experiment to experiment. In both these cases the properties of the fluid are determinate. In turbulent flow however the property would be fluctuating in time and botwen experiments and would appear random in nature. So we can say that turbulent flow does not appear determinate in any simple sense. However, turbulent flows and their effects are commonly encountered in most cases of fluid motion. It is true to say that one is more likely to meet turbulent flow than laminar flow in most engineering situations. So it becomes important to know how turbulent flow behaves. At the same time it is necessary tn know why they behave the way they do in order to formulate rules for practical use and, with a basic understanding of the phenomenon, to propose methods for its application and control. 
Turbulence is three-dimensional, non-linear and diffusive. Diffusion in turbulence accounts for the differing characteristics of, for instance, mean velocity distributions and heat transfer between laminar and turbulent flow. In the kinetic theory of gases the diffusion of molecules is explained in terms of the random motion of the molecules, where a molecule, by a process of random walk, moves farther and farther from its point of departure. Turbulent diffusion may also be likened to a random walk but now the translations and collisions occur between separate bodies of fluid.

The non-linear character of turbulence presents the hardest problem in any attempts at its understanding. The ivavier Stokes equations, which, it is generally accepted, adequately describe the fluia motion in terms of mean and fluctuating components, are non-linear. Although a complete solution of these equations is possible on a high speed digital computer, the process would take a long time and it is questionable whether the information gathered would be worth the effort. What part does non-linearity play in the phenomenon of turow bulence? One clear fact that emerges from experiments is that the magnitude of the turbulent fluctuations is an order of magnitude smaller than that of the mean flow. So it seems reasonable to argue that there exists some process in the How that limits the amplitude of the fluctuations. However, in order to maintain the turbulence, there must exist a process for the generation and growth of the fluctuations. It 
is the author"s view that both these mechanisms are best assigned to non-linearity. However, there exists no widely accepted view on the role of the non-linear actions. If non-linear effects are entirely daxping effects, what mechanism is there to prevent the turbulence being in a continual state of decay and, if that is the case, how can disturbances grow? So one might reasonably argue that small disturbances are amplified, by a linear or non-linear process, to some amplitude where non-linearity prevents their further growth.

The very nature of turbulence, its three-dimensionality and its randomess, has made experiments in this field highly complicated. At the same time the techniques developed to make these measurements have been ingenious and sophisticated. Turbulence occurs in very many flow situations but the number of flow configurations considered in experiments is relatively few. Because of this there has been a great deal of duplication or measurements. In many instances the apparently differing results from seemingly identical experiments has often initially led more to confusion than to enlightenment. However, the accuracy of experiments is continually increasing as the techniques become more sophisticated, and the results of these experiments are invaluable in any attempts to understand the mechanisms in turbulence.

The description of turbulent motion as a function of space and time is made nearly impossible by its complicated nature. However, it is possible to regard turbulerce as a phenomenon in which the fluctuating quantities are to have 
random variations with space and time. In this way it becomes posisible to describe the turbulence in terms of a number of statistical propertios. Tho statistical theory of turbulenco was initiated by GoI. Taylor (1935). Subsequent stujies of the structure of turbulent shear flow have been greatly influenced by the statistical theory as it presented the possibility of describing the turbulent motion analytically from the equation of motion without arbitrary assumptions. Much experimental work has thus been directed at measuring statistical properties of the turbulence such as velocity-velocity correlations and root mean squares of the flow properties. The measurement of these properties enables the researcher to gain a clearer understanding of the physical nature of the flow. Advanced masurement techiques have recently enabled workers to measure space and time correlations and power spectra of turbulent velocity components in many different shear flows。 Notakle contributions have been made by Favre, Gaviglio and Dumas (1958) for the turbulent boundary layer, Grant (1958) for boundary layers, wakes and grid turbulence, and Bradshaw, Ferriss and Johnson (1964), for a circular jeto The measurement of fluctuating pressures in turbulence presents more cxperimental problems than the measurement of velocity fluctuations; however, experiments have been carried out by Fuchs (1968) for a jet and in the boundary layer correlations between wall pressure fluctuations and velocity components have been made by Hodgson (1962) and Thu and Willmorth (1966)。 
Thus it can be seen that, with the introduction of more sophisticated techniques such as conditional sampling for the measurement of intermittancy, see Wygnanski and Fiedler (1970), experimental investigations have reached a very advanced state。 Theoretical investigations have not advanced as quickly as experimental work in describing the nature of turbulent motion. Much of the work has been applied to isotropic and homogeneous turbulence, most of which work is described by Batchelor (1953)。The extension of this work to inhomogeneous turbulence has led to great difficulties and one is often forced to empirical hypotheses. Fundamental to the description of turbulence is the description of the mechanism by which the Reynolds shear stress is maintained. Many of the early theories of turbulent flow were of a phenomenological nature and described the shear stress in terms of a mixing length or an eddy viscosity. Assuming a similarity condition the equations of motion could then be solved for distribution of mean velocity。 Only Reichardt pointed out that the comparison of the results of the various theories with experimental data, which usually contained a lot of scatter, would not allow a decision to be made in farour of any particular theory. He proposed an inductive theory, described by Abramovitch (1963), based on the results of experimental investigations of directly measurable quantities such as the mean square of the axial velocity fluctuations. The assumption of a constant eddy viscosity has led to a number of useful predictions, however it is clear that the shear stress is not a local property 
of the flow but a function of the whole flow fiela.

The next major development, subsequent to the phenomenological theoxies and statistical theories, was made by Townsend (1956) with the publication of his monograph, "The Structure of Turbulent Shear Flow". He presented an inductive account of the processes involved in turbulent shear flows. He proposed that the turbulence could be separated into various scales of eddy motion. The medium scale eddies would contain almost all of the turbulent energy. The large eddies would be responsible for the distortion of the turbulence front and would control the entrainment of irrotational fluid. They would also be responsible for the intermittent nature of the turbulence. In the same year another new development was proposed by Malikus (1956). Malkus studied the turbulent shear flow in a channel without any empirical assumptions. In his theory he assumed that the non-linear momentum transport, had only a stabilising effect on the mean flow. His hypcthesis may be stated that firstly, the mean flow will be stable if perturbations of the mean flow are marginally stable and satisfy the Oxr-Sommerfeld equation, and secondly, that the scale of these marginally stable modes will be the smallest scale present in the momentum transport spectrum. These hypotheses mean that the mean profile will be marginally stable in an Orr-Sommerfeld sense and that the Reynolds shear stress is composed of scales larger then the smallest scale in the spectrum of momentum transport. The equation 
of mean motion for channel flow may be written

$$
\frac{\partial}{\partial y} \overline{u v}=\frac{\tau_{0}}{a}+v \frac{\partial^{2} U}{\partial y^{2}}
$$

where $\tau_{0}=-\frac{a}{\rho} \frac{\partial P}{\partial x}$, where $P$ is the wall pressure and $d$ is the separation of the walls.

We may write $\overline{\text { uV }}$ as a series of oxthogonal functions where the last term in the series corresponds to the smallest scale of the fluctuations. Thus we write,

$$
\overline{u v}=\sum_{n=1}^{n_{0}} A_{n} \sin \frac{2 n \pi y}{d}
$$

Substituting the series (1.2) into equation (1.1) leads to,

$$
\frac{d^{2} U}{d y^{2}}=\sum_{n=1}^{n_{0}} B_{n} \cos \frac{2 n \pi y}{d}-\frac{\tau_{0}}{v d}
$$

Assuming that $\frac{d^{2} U}{d y^{2}}$ is positive definite we may guard against the possibility of inflectional instability. Malkus then proposed to find the mean profile which would make the rate of dissipation of potential energy into heat a maximum. This condition would then $f^{\prime} i x$ the value of $n_{0}$. The rate of dissipation of energy by viscosity will equal the rate of mechanical work cone by shearing stresses. The total rate of dissipation per unit mass is given by

$$
D=\tau_{0} U_{m}
$$

where $U_{m}$ is the $y$-averaged mean velocity which is the reference velocity. We need to find the conditions for maximum $D$, which 
will clearly occur for maximum $\tau_{0}, U_{m}$ being fixed. Thus we consider the maximum value of the non-dimensional quantity $\frac{R t_{0}}{U_{m}^{2}}$, where $R=\frac{U_{m}}{\nu}$ is known. Alternatively, we nay $k \in e p$ this quantity constant and find the minimum Reynolds number associated with the marginally stable solution of the OrrSommerfeld equation. This then fixes the value of $\mathrm{n}_{0}$ which gives the last term in the series for $\overline{u v}$. The subsequent determination of the mean velocity profile through optimisation of the dissipation rate involves much complicated analysis. However, the solution obtained by Malkus was in close agreement with the measurements of Laufer (1951) and is the only profile obtained on purely theoretical analysis.

The success of Malkus theory has led a number of researchers to analyse his hypothesis. Townsend (1962) reviewed Malkus work and discussed its physical implications. We may note that the total energy dissipation is determined by the velocity gradient at the walls. As the Reynolds number of the flow tends to infinity the velocity profile at the channel centre becomes flatter and approaches a constant value, thus maximum dissipation will occur for infinite Reynolds number. Townsend also noted that the modes considered by Malkus obtained their energy directly from the mean motion but the energy was lost to a background of isotropic edaies by a non-linear transfer process supporting the idea of the stabilising effects of non-linear phenomena. 
More recently Reynolds and Tiedeman (1967) contradict the Malkus theory. As a result of extensive numerical calculations they conclude that the experimental profiles were stable in linear perturbation theory and the marginally stable ones do not correspond with experiment. They could not find any neutral stability curve in this case. However Eckhaus (1965) points out that in the classical calculation of critical curves it is considered that if other eigenvalues than the ones considered exist, then they are all stabilising. He also notes that unless there exists an infinite denumerable sequence of eigenvalues in the case of Poiseville flow such that the $(n+1)$ th eigenvalue is more stable than the $n-t h$, then no critical curve may exist. The number of eigenvalues of the Orr-Sormerfeld equation was considered by Corcos and Sellars (1959) for the case of Poisecille flow in a circular pipe. They concluded that, as for every fixed value of Reynolds number and wavenumber the number of eigenvalues was finite, the number of eigenvalues is finite. Eckhaus extends this argument to state that as the Reynolds number tends to infinity the number of eigenvalues is finite for each fixed value of $R$ but increases without limit as $R \rightarrow \infty$. We will now consider other lines of recent research on the problem of turbulent shear flow.

We have previously noted that the description of the Reynolds shear streis is fundamental to study of turbulent shear flows. Phillips (1967) presented a hypothesis for the gradient of Reynolas shear stress as a function of the 
convected integral time scale. The generation of Feynolds shear stress is generated by the interaction of the fluctuations and mean velocity field. The interaction between uniform shear and a single Fourier component of the turbulent flow has been considered by, amongst others, Moffatt (1965)。 He concluded. that, in an analysis which neglected the interaction between separate fluctuations, there existed no unique relationship between the local mean velocity gradient and the Reynolds stress. This supports the idea that the Reynolds stress is not a "local" property of the turbulent field but a property of the whole field. We may simply show that the Boussinesq hypothesis of constant eddy viscosity is not valid. He hypothesised that the shear stresses would be proportional to the local velocity gradient. He wrote

$$
-\overline{u_{i} u_{j}}=v_{T}\left(\frac{\partial U_{i}}{\partial x_{j}}+\frac{\partial U_{j}}{\partial x_{i}}\right),
$$

where $v_{T}$ is the eddy viscosity and $U_{i}$ and $u_{i}$ are the mean and fluctuating components of velocity in the $x_{i}$-direction. If we consider the case of $i=j$ in $(1.4)$ we see that for an incompressible fluid,

$$
-\bar{u}_{i} u_{i}=2 v_{T} \frac{\partial U_{i}}{\partial x_{i}}=0,
$$

Which implies no turbulence exists in incompressible flow。 Phillips noted that experiments show that the energy containing turbulent components, which generate the Reynolds stress, have scales of the same order as the mean flow. He 
pointed out that it is the variation of Reynolds stress that occurs in the momentum equation and he argued that one should consider the relation between variation in the ReynoLas stress and variations in the mean velocity gradient. A similar association was found by Miles (1957) in the case of inviscid laminar air flow over water waves. He found that the stress difference across the critical layer, where the wave speed equals the wind speed, is proportional to the ratio of local mean velocity curvature to its slope. Phillips derived a similar expression for the contribution to the shear stress across the "matched layex", where the mean velocity is equal to the wave speed. He wrote this as

$$
\Delta \tau_{p}=A_{m} \rho\left\{\frac{-U^{\prime}}{k U^{q}} \overline{W^{2}}\right\}_{z_{m}},
$$

where $A_{m}$ is a numerical constant and the term in brackets is evaluated at the matched layer where $z=z_{m}$. $\overline{W^{2}}$ is the mean square periodic perturbation in the $z$-direction. He then considered the turbulent motion in a Fourier decomposition as the superposition of a large number of small travelling perturbations, periodic in space in the $x-y$ plane. By considering the contribution from all the wavenumers of the turbulence with their matched layers at $z_{m}$ he derived the expression for the gradient of the Reynolds stress,

$$
\left.\frac{d \tau}{d z}=A \text { A }\right) \overline{w^{2}} \frac{d^{2} U}{d z^{2}} \text {, }
$$

where $A$ is a number, (A) is the convected integral time scale 
of the $W$-velocity fluctuations and $U(z)$ is the mean velocity profile. Thus we may write the eddy viscosity

$$
v_{\mathrm{r}}(\mathrm{z})=\mathrm{A} \text { (⿴⿱冂一⿱一一厶) } \overline{w^{2}}
$$

From equations $(1.8)$ and $(1.9)$ we see that the Reynolds stress is not a local property of the flow. Although the Reynolds stress gradient would appear to be a local function of the curvature of mean velocity and velocity fluctuations but the convected time integral scale involves a history of the turbulence. We also see the importance of the energy containing eddies in supporting the Reynolds stress as the eddy viscosity is proportional to kinetic energy density of the W-velocity fluctuations. The inclusion of the convected integral time scale shows that the longer the $W$-fluctuation remains coherent the greater their contribution to the shear stress。 However, Hall (1968) has pointed out that if Phillips' expression is integrated across a jet or wake momentum will not be conserved which suggests that Phillips hypothesis may not be useful in these cases.

Another formulation of the problem has been given by Lilley. He compared the turbulence terms in the vorticity equation with those in the equations of motion and derived an expression in twomimensional flow which is given by,

$$
-\overline{u_{1} u_{2}}=\int \overline{\omega_{3} u_{2}} d x_{2}
$$

where $\omega_{3}$ is fluctuating vorticity in two dimensions. In a 
manner similar to Phillips we note that the Reynolds stress is a global property of the flow and also that it obtains its value from regions of high vorticity production. Lilley (1967) observed that it would be possible to write,

$$
\overline{\omega_{3} u_{2}}=-A e^{2} \frac{\partial \Omega}{\partial x_{2}}\left(\overline{u_{2}^{2}}\right)^{\frac{3}{2}}
$$

where $A$ is an arbitrary constant, $\Omega$ is the mean vorticity and $l$ is a mixing length. From equation (1.10) we see that,

$$
-\overline{\mu_{1} \mu_{2}}=\int A e^{2} \frac{\partial \Omega}{\partial x_{2}}\left(\bar{\mu}_{2}^{2}\right)^{\frac{1}{2}} d x_{2}
$$

The right hand side may be integrated numerically with the measurements of root mean square velocity fluctuations and the mear velocity profile. We may note that in Phillips' formulation of the problem the Reynolds stress is related to the kinetic energy of the transverse velocity fluctuations and in Lilley"s formulation it is related to the fluctuating vorticity. Towards the edge of the mixing layer, or the free edge of any shear flow, the value of $\bar{u}_{1} u_{2}$ falls rapidly to zero as does the value of $\overline{\omega_{3}^{2}}$ whereas the kinetic energy of the fluctuations remains small but finite. Thus a system connecting shear stress to fluctuating vorticity is more reasonable. Sharma (1968) noted that as well as not meeting the requirement of conservation of momentum in the jet or wake, Philitips expression may not be useful in the logarithmic region of the boundary Iayer as here the inverse of $x_{2}$ will appear on the right hand side of his expression and will give very large values of $\overline{u_{1} u_{2}}$ 
for small values of $x_{2}$. However, we see that both the expressions of Iilley and Phillips are similar in nature and in both cases empirical values have to be found for constantso An entirely different formulation of the problem has been suggested by LandahI ( 1967 ). He considered that the turbulent motion in a shear flow may be examined as a wavempopagation phenomenon. Making use of a Fourier transform technique he derived an equation of the Orr-Sommerfeld type for the turbulent fluctuations. His numerical solution of the linearized equation showed that, for infinitessimal disturbances, the turbulent boundary layer with zero pressure gradient is stable. We will now briefly summarise his analysis. First let us consider a wave-guide with stationary random disturb ances. If we call the random signal $p(x, t)$ and assume homogeneity in $\mathrm{x}$ then we may introduce the Fourier transform $p(k, w)$ defined by

$$
p(k, \omega)=\int_{-\infty}^{\infty} \int_{-\infty}^{\infty} e^{-i(k x-\omega t)} p(x, t) d x d t,
$$

where $\omega$ is the frequency and $k$ is the wave number. The wavenumber frequency spectrum, $\hat{S}_{\mathrm{pp}}(\mathrm{k}, \mathrm{w})$, being defined as the Fourier transform of $\mathrm{S}_{\mathrm{pp}}$, the cross spectral density, may then be written,

$$
\hat{S}_{p p}(k, w)=\frac{\overline{p(k, \omega) \beta^{*}\left(k^{2}, \omega^{1}\right)}}{\delta\left(k-k^{3}, w-w^{p}\right)}
$$

where $\delta$ is the generalized Dirac delta function and the star denotes the complex conjugation. The bar denotes an ensemble average. The inverse Fourier transform of (1.14) leads to the cross spectral density function such that, 


$$
S_{p p}(\xi, \omega)=\frac{1}{2 \pi} \int_{-\infty}^{\infty} e^{i k \xi} S_{p p}^{\infty}(k, \omega) d k
$$

The random disturbances will excite the wave modes in the wave-guide and thus the Fourier transform of $p(x, t)$ will contain terms of the form,

$$
\frac{c(n)}{k-\alpha}
$$

where $\alpha^{(n)}=\alpha_{R}^{(n)}+i \alpha^{(n)}$ are the wave propagation constants. In order to ensure that the disturbance field is statistically homogeneous in $x$, it is assumed that all the waves are damped, and hence $\alpha_{I}(n)$ is positive for waves travelling in the positive $x$-direction. This condition is essential in the Iinear model, however statistical homogeneity may be preserved for amplifying disturbancos when non-linear amplitude limiting processes occur. For the case of lightly damped wave modes, which will correlate over the largest distance, where $\left|\alpha_{I}{ }^{(n)}\right| \ll\left|\alpha_{R}{ }^{(n)}\right|$ then the cross spectral density will be of the form,

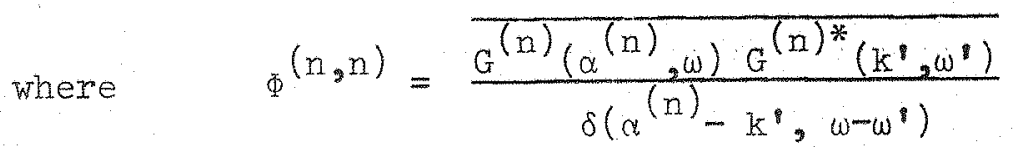

$$
S_{p p}(\xi, \omega)=\sum_{n} \frac{I}{2 \alpha_{I}(n)} \Phi^{(n, n)}(\omega) \exp \left(i \alpha_{R}^{(n)}{ }_{\xi-\alpha_{I}}^{(n)}|\xi|\right),(I . I 7)
$$

and the coefficients $G^{(n)}$ are related to the coefficients $c^{(n)}$ in equation (1.16) and are functions of wave number and frequency. 
In order to evaluate the term $\phi^{(n, n)}$ in equation ( 1.3 .8$)$ Landahl introduces infinitessimal disturbances into the equations of motion and after employing a Fourier transform technique he obtains the equation,

$$
\begin{aligned}
& (U-c)\left(\phi^{B}-k^{2} \phi\right)-U^{\prime} \phi+\frac{i}{\alpha R}\left(\phi^{i v}-2 k^{2} \phi+k^{4} \phi\right) \\
& =a / \alpha k,
\end{aligned}
$$

where $\phi$ is the Fourier transform of the disturbance stream function, $U$ is the mean velocity, $k=\left(\alpha^{2}+\beta^{2}\right)^{\frac{1}{2}}$, where $\alpha$ and $\beta$ are the non-dimensional wave numbers in the $x$ - and $z^{\text {marections }}$ respectively. $\dot{q}$ is the Fourier transform of the non-linear Reynolds stress terms and is regarded as known and will be considered as a forcing function. The main contribution to the cross spectral density is assumed to come from the least attenuated mode and then $\mathrm{S}_{\mathrm{pp}}(\xi, \boldsymbol{\zeta}, \omega)$, for three dimensional disturbances may be written,

$$
\begin{aligned}
S_{p p}(\xi, \zeta, \omega)= & \frac{1}{2 \pi} \int_{-\infty}^{\infty} \frac{\Phi}{2 \alpha_{I}(0)} \exp \left(i \alpha_{R}{ }^{(0)} \xi-\alpha_{I}{ }^{(0)}|\xi|\right. \\
& +i \beta \zeta) d \beta
\end{aligned}
$$

Landahl then notes that the main contribution to the integral in $(1.19)$ will come from the $\Phi^{(0,0)}$ terms as the ejgenvalues are only slightly sensitive to the wave angle. He $i$ is then able to express the cross spectral density in terms of expressions which are independent functions of the longitudinal and transverse separations and he writes this in the form, 


$$
S_{\mathrm{pp}}(\xi, \zeta, \omega)=S(\omega) \exp \left(\frac{i \omega \xi}{U_{C}}\right) A\left(\frac{\omega \xi}{U_{C}}\right) B\left(\frac{\omega \zeta}{U_{C}}\right)
$$

where $U_{C}$ is the convection velocity given by,

$$
U_{C}=\omega / \alpha_{R}
$$

This expression (1.20) was proposed by Corcos (1964) on the basis of the experiments by WiIImarth and Wooldridge (1962). WiIImerth and Roos (1965) showed that there existed regions of separation and non-dimensional frequency where Corcos' similarity formulation does not hold. It is not valid for $\omega \delta^{*} / \mathrm{U}_{\infty}>3,|\zeta| / \delta^{*}<0.7$ and for $\omega \delta^{*} / \mathrm{U}_{\infty}<1,|\zeta| / \delta *>1$.

In his calculations of convection velocity Landahl's equation (1.21) means he has equated convection velocity and phase velocity. This convection velocity was found to be too low by $30 \%$. However, if a viscosity of a much higher value was assumed the convection velocities agreed with experiments. Landahl concluded that one should consider the propagation of a wave in a flow with pre-existing fluctuations, which may act like an eddy viscosity。 However, the inclusion of an eddy viscosity means the problem must be reformed as, for instance, the mean velocity will be a, function of the disturbed flow。

In Landahl's analysis we also see that the ratio $\alpha_{I} / \alpha_{R}$ is taken as constant though it would appear that the variation of $\alpha_{I}$ with frequency is much greater than that of $\alpha_{R}$. LandahI chooses a constant value of $\alpha_{I} / \alpha_{R}$ as he notes 
that the calculated decay rate has an uncertainty, the major contribution to which coming from the uncertainty in the mean velocity profile. This implies that an eddy will decay in a distance proportional to its size. The calculations of Sharma (1968) show that the ratio $\alpha_{I} / \alpha_{R}$ is not constant and the proportional rate of decay of small edaies is much greater than that of the larger eddies. Landahl's results also indicate that the wave number/frequency ratio is constant, which implies that the system is non-dispersive.

The non-linear terms in Landahl's work are treated as a forcing function needed to maintain an otherwise continuously decaying disturbance field. It is clear that this assumption will not hold when there exists an unstable mode in the flow: It is Iikely that turbulent bursts in the boundary layer, which Landahl associates with a non-linear process, are the result of instabilities in the mean profile. Experiments indicate that these bursts occur in the region where large variations occur in the derivatives of the mean velocity profile and it is this, as Landahl notes, which leads to uncertainty in his calculations of the amplification factors. Landahi also relies on there only being one dominant mode in the flow which is not the case for instance in the jet, Where Lessen et al (1965) found more than one unstable mode.

It would appear that the range of application of Landahl"s hypothesis is limited to flows where there exist no amplifying disturbances. In these cases the assumption that non-iinear 
terms are forcing functions is more reasonable. This is only possible in certain cases in the light of a linear analysis. For the case of the boundary layer, Sharma (1968) found that there are amplifying modes. We may also recall that Malkus hypothesised that the non-linear terms have a stabilising influence on the flow.

In our present study of the turbulent mixing layer between two streams will we have need to call on the nonIinear terms to act as both stabilising and destabilising influences. In order to present a complete study of the mixing layex the work will be divided into four sections. We will firstly consider the solution of the mean equations of motion for both cases of laminar and turbulent mixing. Secondly, we will examine the stability of a laminar mixing layer between two streams of finite velocity. We will then present a model for the structure of the turbulent mixing Layer. Finally, the effects of finite amplitude disturbances in the mixing layer will be examined. A review of previous work on these various topics will be presented in the relevant sections.

In the next chapter we will derive the governing differential and integral equation of the mixing layer between two streams. 


\section{CHAPTER 2}

\section{Mathematical Derivation}

In this chapter the equations that describe the turbuIent shear flow problem will be described. These equations will be presented in a convenient form for the performance of numerical calculations.

\subsection{Governing Differential Equations}

For convenience we will derive the equation using tensor notation. Repeated suffices will imply summation over the values of the repeated suffix. A point in the flow is written as $x$ with co-ordinates $x_{i}$ where, for rectangular cartesian coordinates, $i=1,2,3$ and the velocity component of the flow in the $x_{i}$ direction is given by $u_{i}$. In certain sections of the analysis the notation $(x, y, z)$ and $(u, v, w)$ will be used instead of $x_{i}$ and $u_{i}$ respectively.

It is initially assumed that the fluid is incompressible and that the fluid properties are independent of temperature. Thus fluctuations of fluid density due to pressure, entropy and vortical fluctuations are neglected. These assumptions are valid if the flow speed is low. When calculations of the acoustic radiation of the flow are to be made the compressible Plow equations must be used. However, the incompressible flow problem will be solved as the starting point for dealing with the complete problem.

The equation of continuity for incompressible flow may be written,

$$
\frac{\partial u_{i}}{\partial x_{i}}=0
$$


The Navier-Stokes equations will be assumed to describe the motions of both turbulent and laminar flow. Thel may be written for turbulent incompressible flow as,

$$
\frac{\partial u_{i}}{\partial t}+u_{j} \frac{\partial u_{i}}{\partial x_{j}}=-\frac{I}{\rho} \frac{\partial p}{\partial x_{i}}+v \nabla^{2} u_{i}
$$

Using the equation of continuity (2.1), equation (2.2) may be rewritten,

$$
\frac{\partial u_{i}}{\partial t}+\frac{\partial u_{i} u_{j}}{\partial x_{j}}=-\frac{1}{\rho} \frac{\partial p}{\partial x_{i}}+\nu \nabla^{2} u_{i}
$$

where $i, j=1,2,3,\left(x_{1}, x_{2}, x_{3}\right)$ are a set of orthogonal coordinates, $\nabla^{2}=\frac{\partial^{2}}{\partial x_{1}^{2}}+\frac{\partial^{2}}{\partial x_{2}^{2}}+\frac{\partial^{2}}{\partial x_{3}^{2}}, u_{i}$ is the instantaneous velocity component parallel to the $x_{i}$ axis, $\rho$ is the fluid density, $p$ is the pressure and $v$ is the kinematic viscosity, $u / \rho$ 。

The instantaneous values of velocity and pressure may be written as,

$$
\begin{array}{ll}
u_{i}\left(x_{i}, t\right)=u_{i}\left(x_{i}\right)+u_{i}^{\prime}\left(x_{i}, t\right) & ) \\
p\left(x_{i}, t\right)=P\left(x_{i}\right)+p^{\prime}\left(x_{i}, t\right) & )
\end{array}
$$$$
\text { where } u_{i} \text { ' and } p^{\prime} \text { are the turbulent fluctuations and } U_{i} \text { and } P
$$$$
\text { are the average values of velocity and pressure averaged }
$$
accoxding to,

$$
U_{i}=\operatorname{lin}_{T \rightarrow \infty} \frac{1}{2 T} \int_{-T}^{T} u_{i} d t \text {, etc. }
$$

By substitution of the relations (2.4) into (2.2) and (2.3) and averaging over a long period, we have, 


$$
\frac{\partial U_{i}}{\partial x_{j}}=0,
$$

and

$$
\frac{\partial U_{i} U_{j}}{\partial x_{j}}+\frac{\overline{\partial u_{i}^{i} u_{j}^{i}}}{\partial x_{j}}=-\frac{1}{\rho} \frac{\partial P}{\partial x_{i}}+v \nabla^{2}\left(U_{i}\right)
$$

When the equations for the mean flow (2.5) are subtracted from equations (2.1) and (2.3) we obtain the equations for the fluctuations,

$$
\begin{aligned}
& \frac{\partial u_{i}^{\prime}}{\partial x_{i}}=0, \\
& \frac{\partial u_{i}^{!}}{\partial t}+\frac{\partial u_{i}^{!} U_{j}}{\partial x_{j}}+\frac{\partial u_{i} u_{j}^{\prime}}{\partial x_{j}}+\frac{\partial u_{i}^{\prime} u_{j}^{!}}{\partial x_{j}}-\frac{\partial u_{i}^{!} u_{j}^{!}}{\partial x_{j}}=-\frac{1}{\rho} \frac{\partial p^{\prime}}{\partial x_{i}}+v \nabla^{2}\left(u_{i}^{!}\right)
\end{aligned}
$$

If it is argued that the disturbance motion is rotational it is convenient to rewrite the equations in terms of a fluctuating vorticity. Thus on making use of the alternating, thirdorder tensor, $\varepsilon_{i j k}$,

where (i) $\varepsilon_{i j k}=0$ if any two of the indices $i j k$ are equal,

$$
\text { (ii) } \varepsilon_{123}=\varepsilon_{231}=\varepsilon_{312}=1 \text {, }
$$

and (iii) $\varepsilon_{132}=\varepsilon_{321}=\varepsilon_{213}=-1$, equations (2.2) may be written in terms of the vorticity. Helmholtz's equations for the vorticity follow and are,

$$
\frac{\partial \omega_{i}}{\partial t}+u_{j} \frac{\partial \omega_{i}}{\partial x_{j}}=w_{j} \frac{\partial u_{i}}{\partial x_{j}}+v \nabla^{2} \omega_{i},
$$

where the vorticity, $\omega_{k}$, is given by,

$$
\omega_{k}=\frac{\partial u_{i}}{\partial x_{j}} \varepsilon_{i j k}
$$


Now since the divergence of the vorticity is zero, or in tensor notation,

$$
\frac{\partial \omega_{i}}{\partial x_{i}}=0
$$

then equation (2.7) may be rewritten,

$$
\frac{\partial \omega_{i}}{\partial t}+\frac{\partial \omega_{i} u_{j}}{\partial x_{j}}=\frac{\partial u_{i} \omega_{j}}{\partial x_{j}}+\nu \nabla^{2} \omega_{i}
$$

If the instantaneous value of the vorticity is written as,

$$
\omega_{i}\left(x_{i}, t\right)=\Omega_{i}\left(x_{i}\right)+w_{i}^{!}\left(x_{i}, t\right),
$$

then substitution of this relation into equation (2.9) and averaging over a long time period yields the equation for the mean vorticity,

$$
\frac{\partial \Omega_{i} U_{j}}{\partial x_{j}}-\frac{\partial W_{i} j}{\partial x_{j}}+\frac{\partial \overline{\omega_{i}^{i} u_{j}^{q}}}{\partial x_{j}}-\frac{\partial \overline{u_{i}^{!} \omega_{j}^{q}}}{\partial x_{j}}=v \nabla^{2}\left(\Omega_{i}\right) .
$$

To obtain the equation for the vorticity fluctuations equation (2.10) is subtracted from equation (2.7) to yield,

$$
\begin{aligned}
& \left.\frac{\partial \omega_{i}^{\prime}}{\partial t}+\frac{\partial U_{j} \omega_{i}^{\prime}}{\partial x_{j}}+\frac{\partial \Omega_{i} u_{j}^{!}}{\partial x_{j}}-\frac{\partial \Omega_{j} u_{i}^{!}}{\partial x_{j}}-\frac{\partial U_{i} \omega_{j}^{q}}{\partial x_{j}}+x_{i}=\nu \nabla^{2}\left(\omega_{i}^{q}\right)\right) \\
& \text { where } \left.x_{i}=\frac{\partial\left(u_{j}^{q} \omega_{i}^{!}-u_{i}^{\prime} \omega_{j}^{\prime}\right)}{\partial x_{j}}-\frac{\partial\left(\overline{u_{j}^{l} \omega_{i}^{\prime}}-\overline{u_{i}^{!} \omega_{j}^{\prime}}\right)}{\partial x_{j}}\right)
\end{aligned}
$$

Having derived the general differential equations which describe the motion of a turbulent fluid, the next sections will consider their application to the problem of the turbulent mixing of two parallel streams. Firstly, the equations that describe 
the stabiljty of the flow will be derived. The integral

equations of the mean flow and the fluctuations will be derived and the significance of the components in the equations will be discussed.

\subsection{Derivation of the Equations for the Stability of the Viscous Shear Layer}

The study of hydrodynamic stability was initiated with the work of Helmholtz, (1868). This contribution was followed by the works of Reynolds (1883), Kelvin (1880) and Rayleigh (1878 onwards). The derivation of the equations which included the effects of viscosity and enabled a critical Reynolds number to be calculated, was arrived at independently by Orr (1907) and Sommerfeld (1908). The Orr-Sommerfeld equation was derived by considering two-dimensional wave-like disturbances superimposed on the parallel mean flow. The equation remained unsolved for a number of years until Tollmien (1929) obtained the first critical Reynolds number. A review of methods of solution of the Orr-Sommerfeld equation is presented later, however we will be concerned here with its derivation for non-parallel flows and three-dimensional disturbances.

\subsection{The Stability of the Shear Layer to Three-dimensional Disturbances}

In this section we will derive the stability equation for a shear layer with two-dimensional mean flow. $x_{1}$ is measured along the line of separation of the two parallel streams prior to mixing, $x_{3}$ is measured in the transverse direction across the plane of mixing and $x_{2}$ is measured normal to $x_{1}$ and $x_{3}$. The origin of co-ordinates is taken as the point at which mixing theoretically commences, see diagram below. 


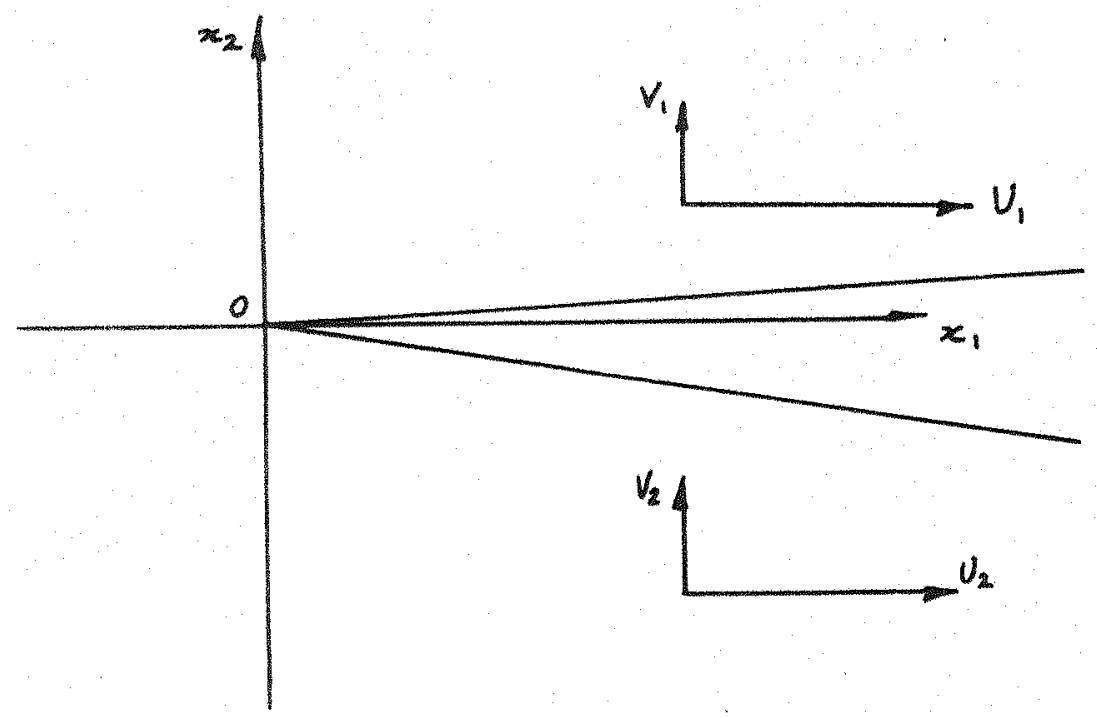

As a consequence of the assumption of a two-dimensional mean flow we have that the mean velocity is a function of $x_{1}$ and $x_{2}$. The assumption of a parallel mean flow will be considered at a later stage when the stability equations in their fuller form have been derived. Thus we may write,

and

$$
\begin{array}{ll}
u_{1}\left(x_{i}, t\right)=v\left(x_{1}, x_{2}\right)+u_{1}^{\prime}\left(x_{i}, t\right) & ) \\
u_{2}\left(x_{i}, t\right)=v\left(x_{1}, x_{2}\right)+u_{2}^{\prime}\left(x_{i}, t\right) & u_{3}^{\prime}\left(x_{i}, t\right) \\
u_{3}\left(x_{i}, t\right)= & \} \\
p\left(x_{i}, t\right)=P\left(x_{1}, x_{2}\right)+p^{\prime}\left(x_{i}, t\right) & )
\end{array}
$$

The corresponding relations for the vorticity components are written,

$$
\left.\begin{array}{rlr}
\omega_{1}\left(x_{i}, t\right) & = & \omega_{1}^{\prime}\left(x_{i}, t\right) \\
\omega_{2}\left(x_{i}, t\right) & = & \omega_{2}^{\prime}\left(x_{i}, t\right)
\end{array}\right)
$$

Substitution of these relations into equation (2.11) and the first of equation (2.6) yields; 


$$
\begin{aligned}
& \left.\left\{\frac{\partial}{\partial t}+\frac{U \partial}{\partial x_{1}}+\frac{v \partial}{\partial x_{2}}\right\} \omega_{1}-\Omega_{3} \frac{\partial u_{1}}{\partial x_{3}}-\omega_{1} \frac{\partial U}{\partial x_{1}}-\omega_{2} \frac{\partial U}{\partial x_{2}}+x_{1}=v \nabla^{2}\left(\omega_{1}\right)\right) \\
& \left.\left\{\frac{\partial}{\partial t}+\frac{U \partial}{\partial x_{1}}+\frac{v \partial}{\partial x_{2}}\right\} \omega_{2}-\Omega_{3} \frac{\partial u_{2}}{\partial x_{3}}-\omega_{1} \frac{\partial V}{\partial x_{1}}-\omega_{2} \frac{\partial v}{\partial x_{2}}+x_{2}=v \nabla^{2}\left(\omega_{2}\right)\right)(2.14) \\
& \left.\left\{\frac{\partial}{\partial t}+\frac{U \partial}{\partial x_{1}}+v \frac{\partial}{\partial x_{2}}\right\} \omega_{3}+u_{1} \frac{\partial \Omega_{3}}{\partial x_{1}}+u_{2} \frac{\partial \Omega_{3}}{\partial x_{2}}-\Omega_{3} \frac{\partial u_{3}}{\partial x_{3}}+x_{3}=v \nabla^{2}\left(\omega_{3}\right)\right) \\
& \text { and } \frac{\partial u_{1}}{\partial x_{1}}+\frac{\partial u_{2}}{\partial x_{2}}+\frac{\partial u_{3}}{\partial x_{3}}=0
\end{aligned}
$$

where

$x_{1}=\frac{\partial\left(u_{2} \omega_{1}-u_{1} \omega_{2}\right)}{\partial x_{2}}+\frac{\partial\left(u_{3} \omega_{1}-u_{1} \omega_{3}\right)}{\partial x_{3}}-\{(\square)+(\square)\}$

$x_{2}=\frac{\partial\left(u_{1} \omega_{2}-u_{2} \omega_{1}\right)}{\partial x_{1}}+\frac{\partial\left(u_{3} \omega_{2}-u_{2} \omega_{3}\right)}{\partial x_{3}}-\{(\square)+(\square)\}$

and

$x_{3}=\frac{\partial\left(u_{1} \omega_{3}-u_{3} \omega_{1}\right)}{\partial x_{1}}+\frac{\partial\left(u_{2} \omega_{3}-u_{3} \omega_{2}\right)}{\partial x_{2}}-\{(\square)+(\square$

where the bracketted terms, ( $)$, represent the time averages of the preceding fluctuating quantities. The primes denoting fluctuating quantities have been omitted for convenience.

In linearised theory it is assumed that, as the fluctuations are infinitesimal, products of fluctuations and their derivatives, such as $\frac{\partial u_{2} \omega_{1}}{\partial x_{2}}$, may be neglected in comparison with the other terms in the equations. In the subsequent analysis we will discard the $x_{1}$ - momentum equation in (2.14) in favour of the equation of continuity. We will further assume that in equations (2.13) the mean vorticity component, $\Omega_{3}$, is dominated by the term $\frac{\partial U}{\partial x_{2}}$. Then the last three equations of $(2.14)$ become, 


$$
\left.\begin{array}{l}
\left\{\frac{\partial}{\partial t}+\frac{U \partial}{\partial x_{1}}+\frac{v \partial}{\partial x_{2}}-v \nabla^{2}\right\} \omega_{2}-\frac{\partial v}{\partial x_{2}} \omega_{2}+\frac{\partial U}{\partial x_{2}} \frac{\partial u_{2}}{\partial x_{3}}=0 \\
\left.\left\{\frac{\partial}{\partial t}+\frac{U \partial}{\partial x_{1}}+\frac{v \partial}{\partial x_{2}}-v \nabla^{2}\right\} \omega_{3}+\frac{\partial U}{\partial x_{2}} \frac{\partial u_{3}}{\partial x_{3}}-u_{2} \frac{\partial^{2} U}{\partial x_{2}^{2}}+u_{1} \frac{\partial^{2} v}{\partial x_{2}^{2}}=0\right\} \\
\text { and } \frac{\partial u_{1}}{\partial x_{1}}+\frac{\partial u_{2}}{\partial x_{2}}+\frac{\partial u_{3}}{\partial x_{3}}=0
\end{array}\right\}
$$

In these equations we have retained those terms in the mean flow that are of order unity or greater according to the normal boundary layer approximation.

In order to reduce these partial differential equations to an ordinary differential equation we will make use of the Fourier transform method. We will assume that the flow is statistically stationary in the $x_{1}-x_{3}$ plane and that the boundary conditions may be written in terms of the transverse distance $\mathrm{x}_{2}$. We will reduce the set of equations $(2.15)$ to a set of ordinary differential equations by using the Fourier transform with respect to $x_{1}, x_{3}$ and $t$. Thus we write, $\hat{q}\left(k, \omega, x_{2}\right)=\frac{1}{(2 \pi)^{3}} \iint_{-\infty}^{\infty} \int_{0} \exp \left\{-i\left(k_{1} x_{1}+k_{3} x_{3}-\omega t\right)\right\}$

$$
q\left(x_{1}, x_{3}, t, x_{2}\right) d x_{1} d x_{3} d t
$$

where $q\left(x_{1}, x_{3}, t_{;} x_{2}\right)$ is a generalised disturbance function dependent on space and time, $\hat{q}\left(\mathrm{k}_{\mathrm{c}}, \omega ; x_{2}\right)$ is its Fourier transform, $k=\left(k_{1}, k_{3}\right)$ is the wave number vector of the fluctuation in the $x_{1}, x_{3}$ plane and $\omega$ is the complex frequency. The Fourier transform for the velocity fluctuation can be written, 


$$
\begin{aligned}
\hat{u}_{1}\left(k, w ; x_{2}\right)=\frac{1}{(2 \pi)^{3}} \iiint_{-\infty}^{\infty} \exp \left\{-i\left(k_{1} x_{1}+k_{3} x_{3}-\omega t\right)\right\} \\
u_{i}\left(x_{1}, x_{3}, t ; x_{2}\right) d x_{1} d x_{3} d t .
\end{aligned}
$$

where $\hat{u}_{i}\left(\frac{k}{v}, \omega_{j} x_{2}\right)$ is the complex Fourier transform of the velocity fiuctuation $u_{i}(x, t)$. The limits of integration over $x_{1}$ would be better considered as $(x,-x)$ with $x \rightarrow \infty$ to guard against the possibility of aivergence of the integral of $\underline{u}$ at infinity.

In equation (2.15) we note that the derivatives of the mean velocities are all with respect to $x_{2}$. In order to facilitate the transformation of the equations we will assume that $U$ and $V$ are functions of $x_{2}$ only bearing in mind the weak dependence of both on $x_{1}$ :

Transformations of the form (2.16a) of the velocity fluctuations are used and equations (2.15) may be written, with the vorticity fluctuations replaced by their velocity components, as follows,

$$
\begin{aligned}
& \left\{\Delta-\frac{\partial V}{\partial x_{2}}\right\}\left(i \beta \hat{u}_{1}-i \alpha \hat{u}_{3}\right)+i \beta \frac{\partial U}{\partial x_{2}} \hat{u}_{2}=0, \\
& \Delta\left(i \alpha \hat{u}_{2}-\frac{\partial \hat{u}_{1}}{\partial x_{2}}\right)+i \beta \frac{\partial U}{\partial x_{2}} \hat{u}_{3}-\frac{\partial^{2} U}{\partial x_{2}^{2}} \hat{u}_{2}+\frac{\partial^{2} V}{\partial x_{2}^{2}} \hat{u}_{1}=0,
\end{aligned}
$$

and $i \alpha \hat{u}_{1}+\frac{\partial \hat{u}_{2}}{\partial x_{2}}+i \beta \hat{u}_{3}=0$,

where $\Delta$ is the operator $\left[i \alpha\left(U-\frac{\omega}{\alpha}\right)+\frac{V \partial}{\partial x_{2}}-\frac{1}{R}\left\{\frac{\partial^{2}}{\partial x_{2}^{2}}-\left(\alpha^{2}+\beta^{2}\right)\right\}\right]$ All quantities have been non-dimensionalised with respect to a reference velocity $U_{0}$, and a reference length $L$. Also $\alpha=k_{I} L$, $B=k_{3} L$ and $R=\frac{U L}{v}$.

We note that 
$\left\{\Delta \frac{\partial}{\partial x_{2}}-\frac{\partial}{\partial x_{2}} \Delta\right\}=-i \alpha \frac{\partial U}{\partial x_{2}}-\frac{\partial V}{\partial x_{2}} \frac{\partial}{\partial x_{2}}$

Eliminating $\hat{q}_{3}$ between the second and third equations of (2.I7) we obtain,

$\left[\Delta \frac{\partial}{\partial x_{2}}+i \alpha \frac{\partial U}{\partial x_{2}}-\frac{\partial^{2} V_{7}}{\partial x_{2}^{2}} \hat{u}_{1}-\left[i \alpha \Delta-\frac{\partial U}{\partial x_{2}} \frac{\partial}{\partial x_{2}}-\frac{\partial^{2} U}{\partial x_{2}^{2}} \hat{a}_{2}=0\right.\right.$. Making use of relation (2.18) we may rewrite this in the form,

$\left[\frac{\partial}{\partial x_{2}}\left(\Delta-\frac{\partial V}{\partial x_{2}}\right)\right] \hat{a}_{1}-\left[i \Delta \Delta-\frac{\partial U}{\partial x_{2}} \frac{\partial}{\partial x_{2}}-\frac{\partial^{2} U_{y}}{\partial x_{2}^{2}} \hat{a}_{2}=0\right.$.

We now eliminate $\hat{u}_{3}$ between the first and third of equation (2.17) to obtain,

$\left\{\left(\alpha^{2}+\beta^{2}\right)\left[\Delta-\frac{\partial V}{\partial x_{2}}\right]\right\} \hat{a}_{1}+\left\{\beta^{2} \frac{\partial U}{\partial x_{2}}-i \alpha\left[\Delta-\frac{\partial V}{\partial x_{2}}\right] \frac{\partial}{\partial x_{2}}\right\} \hat{u}_{2}=0$.

If we differentiate this equation with respect to $x_{2}$ we obtain, $\left(\alpha^{2}+\beta^{2}\right)\left\{\frac{\partial}{\partial x_{2}}\left(\Delta-\frac{\partial V}{\partial X_{2}}\right)\right\} \hat{\theta}_{1}+\left\{\beta^{2} \frac{\partial^{2} U}{\partial x_{2}^{2}}+\beta^{2} \frac{\partial U}{\partial x_{2}} \frac{\partial}{\partial x_{2}}\right.$

$$
\left.-i \alpha\left[\frac{\partial}{\partial x_{2}}\left(\Delta-\frac{\partial V}{\partial x_{2}}\right) \frac{\partial}{\partial x_{2}}\right]\right\} a_{2}=0
$$

Eliminating $\left\{\frac{\partial}{\partial x_{2}}\left(\Delta-\frac{\partial V}{\partial x_{2}}\right)\right\} \hat{u}_{1}$ between equations (2.19) and (2.20) and replacing the operator $\Delta$ we obtain, after some rearrangement,

$$
\begin{gathered}
\left(U-\frac{\omega}{\alpha}\right)\left\{\hat{a}_{2}^{\prime \prime}-\left(\alpha^{2}+\beta^{2}\right) \hat{a}_{2}\right\}-U^{\prime \prime} \hat{u}_{2}+\frac{i}{\alpha R}\left\{a_{2}^{i v}-2\left(\alpha^{2}+\beta^{2}\right) \hat{u}_{2}^{\prime \prime}\right. \\
\left.+\left(\alpha^{2}+\beta^{2}\right)^{2} \hat{a}_{2}\right\} \\
\left.=\frac{i}{\alpha}\left(V \hat{u}_{2} \cdots-\left(V+1 \alpha^{2}+\beta^{2}\right) v\right) \hat{a}_{2}^{\prime}\right\}
\end{gathered}
$$

where primes denote differentiation with respect to $x_{2}$ : 
If the right hand side of equation (2.21) is set equal to zero then we obtain the Orr-Sommerfeld equation for threedimensional disturbances,

$$
\begin{aligned}
\left(U-\frac{\omega}{\alpha}\right)\left\{a_{2}^{\prime \prime}-\left(\alpha^{2}+\beta^{2}\right) \hat{u}_{2}\right\}-U^{\prime \prime} \hat{a}_{2} & +\frac{i}{\alpha R}\left\{\hat{a}_{2}^{i v}-2\left(\alpha^{2}+\beta^{2}\right) \hat{a}_{2}^{\prime \prime}+\right. \\
& \left.+\left(\alpha^{2}+\beta^{2}\right)^{2} \hat{u}_{2}\right\}=0
\end{aligned}
$$

Equation (2.21a) would have resulted from the analysis if an assumption of parallel flow had been made. The coefficients of the terms on the right hand side of equation (2.21) are small compared with the coefficients of the Orr-Sommerfeld equation. It may be argued that the inclusion of these terms in the equation will not greatly affect its solution. However, the divergence of the flow is a necessary factor in the solution, as a parallel flow assumption fails to provide a critical Reynolds number. It will be argued that the effect of divergence of the flow is accounted for best by modifying the eigenvalues found for equation (2.21a). The corresponding eigenfunctions of equation (2.21a) are known to change very slowly with change in eigenvalues and it may be argued that the eigenfunctions corresponding to the modified eigenvalues will closely approximate to those of the unmodified values. We will argue that the flow divergence will affect the energy of the interaction between the disturbances and the mean flow and we will modify the eigenvalues according to this argument.

\subsection{The Integral Equations for the Flow}

The integral equations of the mean flow are obtained by integrating over the transverse co-ordinate, $x_{2}$. The equations 
may then be reduced to ordinary differential equations in $x_{1}$.

We will make use of the boundary layer type approximations, that is,

i) $V / U<<1$

ii) $\frac{\partial}{\partial x} / \frac{\partial}{\partial y} \ll 1$, or the growth of the layer is small.

The mean momentum equation may be obtained from the mean vorticity equation $(2,10)$, which may be rewritten for a two dimensional mean flow as,

$$
\frac{v \partial U}{\partial x}+\frac{v \partial U}{\partial y}+\frac{\partial \overline{u v}}{\partial y}+\frac{\partial}{\partial x}\left(\vec{u}^{2}-\vec{v}^{2}\right)=v \frac{\partial^{2} u}{\partial y^{2}}
$$

Let $\delta_{I}(x)$ be the edge of the mixing layer on the side with velocity $U_{1}$ and $-\delta_{2}(x)$ be the edge on the side with velocity $\mathrm{U}_{2}$. Then integrating equation (2.22) between the edges of the layer we obtain,

$$
\int_{-\delta_{2}}^{\delta_{1}} \frac{\partial}{\partial x}\left[\left(u-U_{1}\right)\left(u-U_{2}\right)+\left(\vec{u}^{2}-\vec{v}^{2}\right)\right] d y+\left[V_{2} U_{1}-U_{2} V_{I}\right]=0
$$

Here we have assumed that the disturbances have zero magnitude at the edges of the layer and we have introduced into the integrand the two zero terms, $-\frac{\mathrm{V}_{1}+\mathrm{U}_{2}}{\partial \mathrm{y}}$ and $\frac{\partial U_{1} \mathrm{U}_{2}}{\partial \mathrm{x}}$. In this way the integrand is zero on both sides of the mixing layer. The equation for the differentiation of an integral may be

$$
\begin{aligned}
& \text { written as, } \\
& \int_{\alpha(a)}^{\beta(a)} \frac{\partial}{\partial a}[f(b, a)] d b=\frac{\partial}{\partial a} \int_{\alpha(a)}^{\beta(a)}[f(b, a)] d b+f(\alpha, a) \frac{d \alpha}{d a}-f(\beta, a) \frac{\partial \beta}{\partial a}
\end{aligned}
$$


Making use of this relation we may rewrite eq. (2.23) as

$$
\frac{d}{d x} \int_{-\delta_{2}}^{\delta_{1}}\left[\left(u-U_{1}\right)\left(u-U_{2}\right)+\left(\vec{u}^{2}-\vec{v}^{2}\right)\right] d y+\left[v_{2} U_{1}-U_{2} V_{I}\right]=0
$$

The mechanical energy equation may be obtained most conveniently for the mixing layer by multiplying equation (2.22) throughout by $U-\frac{\left(U_{1}+U_{2}\right)}{2}$, that is,

$$
\begin{gathered}
U^{2} \frac{\partial U}{\partial x}+\frac{I V U U}{\partial y}-\frac{U\left(U_{1}+U_{2}\right)}{2} \frac{\partial U}{\partial x}-\frac{V}{2} \frac{\partial\left(U_{1}+U_{2}\right) U}{\partial y}+\left[\frac{U-\left(U_{1}+U_{2}\right)}{2}\right] \\
\frac{\partial \overline{u v}}{\partial y}+\left[\frac{U-\left(U_{I}+U_{2}\right)}{2}\right] \frac{\partial\left(\vec{u}^{2}-\nabla^{2}\right)}{\partial x} \\
=v U \frac{\partial^{2} U}{\partial y^{2}}-\frac{v}{2}\left(U_{1}+U_{2}\right) \frac{\partial^{2} U}{\partial y^{2}}
\end{gathered}
$$

Integrating equation (2.26) across the mixing layer we obtain,

$$
\begin{aligned}
& \int_{-\delta}^{\delta_{1}} \frac{d}{\partial x}\left\{\frac{1}{2}\left[U\left(U-U_{1}\right)\left(U-U_{2}\right)\right]+\left[\frac{-\left(U_{1}+U_{2}\right)}{2}\right]\left(\vec{u}^{2}-\vec{v}^{2}\right)\right\} d y \\
& =\int_{-\delta_{2}}^{\delta_{1}}\left(\vec{u}^{2}-\vec{v}^{2}\right) \frac{\partial U}{\partial x} d y+\int_{-\delta_{2}}^{\delta_{1}} \frac{\partial u}{u v} \frac{\partial U}{\partial y} d y-v \int_{-\delta_{2}}^{\delta_{1}}\left(\frac{\partial U}{\partial y}\right)^{2} d y
\end{aligned}
$$

where we have introduced into the integrand the zero term, $\frac{V}{2} \frac{\partial U_{1} U_{2}}{\partial y}$. Making use of relation (2.24) the integral of the mean mechanical energy equation may be written,

$$
\begin{aligned}
& \frac{d}{d x} \int_{-\delta_{2}}^{\delta_{1}}\left\{\frac{1}{2}\left[U\left(U-U_{1}\right)\left(U-U_{2}\right)\right]+\left[U-\frac{\left(U_{1}+U_{2}\right)}{2}\right]\left(\vec{u}^{2}-\vec{v}^{2}\right)\right\} d y \\
& \left.=\int_{-\delta_{2}}^{\delta_{1}}\left(\bar{u}^{2}-\bar{v}^{2}\right) \frac{\partial U}{\partial x} d y+\int_{-\delta_{2}}^{\delta_{1}} \frac{1}{u v} \frac{\partial U}{\partial y} d y-v \int_{-\delta_{2}}^{\delta_{I}} \frac{\partial U}{\partial y}\right)^{2} d y \cdot \quad(2.27)
\end{aligned}
$$


The energy equation for the fluctuation may be obtained by multiplying the second equation of $(2.6)$ throughout by $u_{i}$. We then obtain,

$$
\begin{aligned}
& u_{i} \frac{\partial u_{i}}{\partial t}+\frac{u_{i} \partial u_{i} U_{j}}{\partial x_{j}}+\frac{u_{i}}{\partial U_{i} u_{j}} \frac{u_{i} \partial u_{i} u_{j}}{\partial x_{j}}-\frac{u_{i} \partial \overline{u_{i} u_{j}}}{\partial x_{j}}= \\
& =-\frac{1}{\rho} \frac{\partial p u_{i}}{\partial x_{i}}+v u_{i} \nabla^{2}\left(u_{i}\right)
\end{aligned}
$$

If we introduce the kinetic energy of the turbulenceper unit mass, written as,

$$
\frac{q^{2}}{2}=\frac{u_{i} u_{i}}{2}
$$

then equation (2.28) may be written as, having averaged over a Iong period,

$$
\begin{aligned}
{\left[\frac{\partial}{\partial x_{j}}\left(u_{j} \overline{q^{2}}\right)\right]+\frac{\partial}{\partial x_{j}} } & {\left[\overline{u_{j} \frac{q^{2}}{2}}+\overline{\frac{p u_{j}}{\rho}}\right]=v \nabla^{2} \overline{\frac{q^{2}}{2}}-v \overline{\left(\frac{\partial u_{i}}{\partial x_{j}}\right)^{2}}-} \\
& -\overline{u_{i} u_{j}} \frac{\partial u_{i}}{\partial x_{j}}
\end{aligned}
$$

We will assume that the motion is two-dimensional for mean quantities and time averaged fluctuations. Thus we will neglect $\bar{W}^{2}$ etc. and also assume that $\frac{\partial(\bar{l})}{\partial z}=0$. It will be shown, in our later discussion on the stability of the mixing layer, that the most amplifying disturbances are two-dimensional. Experiments have also shown that in the transition region the disturbances are essentially two-dimensional. Thus, if we confine ourselves in our subsequent analysis to this region of the flow then we may treat the whole flow as two dimensional. If we integrate equation (2.29) over the transverse co-ordinate 
y we obtain,

$$
\begin{aligned}
& \frac{1}{2} \int_{-\delta_{2}}^{\delta_{1}} \frac{d}{\partial x}\left[u\left(\bar{u}^{2}+\bar{v}^{2}\right)+\bar{u}^{3}+\overline{u v^{2}}+\frac{2 \overline{u p}}{\rho}\right] d y \\
& =-\int_{-\delta}^{\delta_{2}}\left(\bar{u}^{2}-\bar{v}^{2}\right) \frac{\partial U}{\partial x} d y-\int_{-\delta_{2}}^{\delta} \overline{u v}\left(\frac{\partial U}{\partial y}+\frac{\partial v}{\partial x}\right) d y \\
& +\frac{v}{2} \int_{-\delta_{2}}^{\delta_{1}} \frac{a^{2}}{\partial x^{2}}\left(\bar{u}^{2}+\bar{v}^{2}\right)-v \int_{-\delta_{2}}^{\delta}\left[\left(\frac{\partial \bar{u}}{\partial x}\right)^{2}+\left(\frac{\partial \bar{u}}{\partial y}\right)^{2}+\left(\frac{\partial v}{\partial x}\right)^{2}+\left(\frac{\partial v}{\partial y}\right)^{2}\right] d y
\end{aligned}
$$

Finally, making use of relationship (2.24), the integral of the mean fluctuation energy equation may be written:

$$
\begin{gathered}
\frac{1}{2} \frac{d}{d x} \int_{-\delta}^{\delta}\left[u\left(\bar{u}^{2}+\vec{v}^{2}\right)+\bar{u}^{3}+\overline{u v^{2}}+\frac{2 u p}{\rho}\right] d y \\
=-\int_{-\delta_{2}}^{\delta_{1}}\left(\bar{u}^{2}-\bar{v}^{2}\right) \frac{\partial U}{\partial x} d y-\int_{-\delta}^{\delta} \bar{u} \overline{u v} \frac{\partial U}{\partial y}-v \int_{-\delta}^{\delta}\left[\left(\frac{\partial u}{\partial x}\right)+\left(\frac{\partial u}{\partial y}\right)^{2}+\right. \\
\left.+\left(\frac{\partial \bar{v}^{2}}{\partial x}\right)^{2}+\left(\frac{\partial \bar{v}}{\partial y}\right)^{2}\right] d y
\end{gathered}
$$

Here we have neglected the terms $\frac{\partial V}{\partial x}$ and $\frac{a^{2}}{d x^{2}} \int\left(\vec{u}^{2}+\vec{v}^{2}\right) d y$ according to our boundary layer type assumptions.

It should be noted that in our derivation of equation (2.3I) the averaging of the fluctuations has been carried out over a long time period assuming that the turbulent field is stationary. In latex analysis, where a single component of frequency will be assumed to exist, the integration will be performed over a single period. 
It is interesting to note briefly the function of the various terms in the fluctuating energy equation. Let us consider equation (2.29). The first term in brackets on the left hand side of the equation represents the change in kinetic energy of the turbulent fiela. The second term represents the aiffusion of the total turbulent energy by the turbulence itself. We may rewrite equation (2.29) as,

$$
\begin{aligned}
{\left[\frac{\partial}{\partial x_{j}}\left(u_{j} \frac{q^{2}}{2}\right)\right] } & +\frac{\partial}{\partial x_{j}}\left[u_{j} \frac{q^{2}}{2}+\frac{p u_{i}}{\rho}\right]=v \frac{\partial}{\partial x_{i}} u_{j}\left(\frac{\partial u_{i}}{\partial x_{j}}+\frac{\partial u_{j}}{\partial x_{i}}\right) \\
& -v\left(\frac{\partial u_{i}}{\partial x_{j}}+\frac{\partial u_{j}}{\partial x_{i}}\right) \frac{\partial u_{j}}{\partial x_{i}}-\overline{u_{i} u_{j}} \frac{\partial U_{i}}{\partial x_{j}}
\end{aligned}
$$

The first term on the right hand side of equation (2.32) represents the work done per unit mass and time by the viscous shear stresses of the turbulence and the second viscous term represents the dissipation per unit mass by the turbulence. The final term describes the transfer of energy between the mean motion and the turbulent shear stresses.

A qualitative analysis of the behaviour of the flow field in a non-linear region may be obtained by considering equation (2.29). The difference between the two small quantities $\vec{u}^{2}$ and $\bar{v}^{2}$ may be assumed to be small. Then we see that when the fluctuations are very small the production term will be negligible compared with the viscous dissipation term and the solution of the equation will be that for a laminar mixing layer. As the fluctuation grows the Reynolds stress term becomes comparable in magnitude with the dissipation term and the mean flow will deviate from the laminar solution. As. the fluctuation amplitude increases further the Reynolds stress 
term dominates and the mean flow then satisfies the solution of the turbulent mixing layer equations.

In the next chapter we will consider the solution of the mean velocity equations for turbulent and laminar mixing layers. These solutions are a required input for the subsequent solution of the small disturbance and stability equations. 


\section{CHAPTER 3}

\section{THY: SOLUTION OF THE MEAN EQUATIONS FOR THE MIXING OF TWO STREAMS}

\subsection{Introduction}

The mixing of two parallel streams can be usefully divided into two types: laminar and turbulent. For both cases of mixing it is possible to deduce that a similar solution will exist. In the case of laminar mixing the rate of spread of the mixing layer is a function of the square root of downstream distance and for turbulent mixing the spread rate is constant. The differential equations for the stream functions, defined in terms of a similarity co-ordinate, may be derived for laminar flow without any assumptions other than the boundary layer type assumptions. For turbulent mixing, however, some hypothesis must be made regarding the behaviour of the Reynolds stress. The inclusion of a stream function in the equations of motion leads in both cases to a third order differential equation. There are thus three boundary conditions required for a unique solution. In this chapter we will derive and solve the differential equations governing the Iaminar and turbulent mixing of two streams.

\subsubsection{The Laminar Mixing of Two Parallel Streams}

The solution of the laminar boundary layer equations for mixing of parallel streams was performed by Lessen (1950) in his stability analysis of a laminar mixing layer. He derived the differential equation for the streamfunction, having assumed a similarity variable, using a general analysis with both undisturbed streams having finite velocity. In order to 
be able to expand a solution of a transformed form of the differential equation it was necessary to limit the boundary conditions such that one stream was at rest. The laminar mixing problem was tackled by Lock (1951) and solved for the case of the mixing of streams of different fluids for various velocity ratios. Lock assumed as a third boundary condition, to be discussed later, that $f(0)=0$ where $f$ is the stream function in terms of a similarity co-ordinate $\eta$. Lin (1953) solved the problem using a modification of Gortler's (1942) method for turbulent mixing. Crane (1957) extended this work to deal with the problem of the compressible mixing of two streams.

It is thought useful at this stage to consider the boundary conditions on the problem. There are two boundary conditions apparent in the problem, namely,

$$
U \rightarrow U_{1} \quad \text { as } \quad y \rightarrow \infty
$$

and

$$
\mathrm{U} \rightarrow \mathrm{U}_{2} \quad \text { as } \quad \mathrm{y} \rightarrow-\infty
$$

The third boundary condition necessary for a unique solution of the third order differential equation of the streamfunction is not so clear. The simplest condition used by $\operatorname{Lin}$ (1953) and Gortler (1942) implied that the $x$-component of velocity along the interface was the mean of the two stream velocities. Iin noted that the choice of the third boundary condition was arbitrary up to a translation along the n-axis. Crane (1957) proposed that the third boundary condition should be of the form $\psi\left(n_{0}\right)=0$ where $n_{0}$ was to be determined by experiment or 
some other means. Kuethe (1935) applied the boundary condition proposed by von Karman based on the balancing of transverse momentum. This was applied to the turbulent case which has the same indeterminateness as the laminar problem. The boundary condition was,

$$
U_{1} V_{1}+U_{2} V_{2}=0
$$

where $V_{1}$ and $V_{2}$ are the mean transverse velocities above and below the mixing layer. Ting (1959) derived the third boundary condition for the zero order solution of the mixing region for several cases of subsonic and supersonic streams. Ting introduced a stretched co-ordinate $Y$ where $Y=\frac{Y}{\varepsilon}$ and $\frac{I}{\varepsilon}$ is of the order of the square root of the Reynolds number. He then showed that the pressure difference across the mixing layer is of order $\varepsilon^{2}$. Thus orientating the velocity profile of the zeroorder solution such that the pressure difference is balanced across the layer led him to the third boundary condition. He concluded that the correct boundary condition for incompressible flow was,

$$
\rho_{0} U_{1} V^{(0)}\left(\frac{x}{L}, \infty\right)+\rho_{0} U_{2} V^{(0)}\left(\frac{x}{L},-\infty\right)=0 \text {, }
$$

where $V^{(0)}\left(\frac{x}{L}, \frac{Y}{L}\right)$ is the zero order transverse velocity in an expansion in terms of $\varepsilon$, which is equivalent to the condition (3.2) proposed by von Karman. A detailed discussion of the third boundary condition is given in Appendix. $I$.

We will now derive the equations for the mean flow in laminar or turbulent mixing. It will be seen that the form of the resulting equations for the similarity stream functions are very similar to each other in both cases of mixing. 


\subsubsection{Derivation of Equations}

We will consider the steady flow of a viscous incompressible fluid. Two uniform streams of velocity $U_{1}$ and $U_{2}$ begin mixing at the point $x=0$. We will assume that the mixing is twodimensional. Introducing the normal boundary layer assumptions, described in the previous chapter, we may write the equations of continuity and momentum,

$$
\begin{array}{cl}
\frac{\partial U}{\partial x}+\frac{\partial V}{\partial y}=0, & \{ \\
\frac{\partial U U}{\partial x}+\frac{V U U}{\partial y}=-\frac{1}{\rho} \frac{\partial P}{\partial x}+\frac{\partial^{2} U}{\partial y^{2}} & \{ \\
\frac{\partial P}{\partial y}=0 & \{
\end{array}
$$

The velocity in the $\mathrm{x}$-airection is assumed to be constant outside the layer where one has $\frac{\partial U}{\partial x}=0$. Thus we may write equations $(3.4)$,

$$
\frac{\partial U}{\partial x}+\frac{\partial V}{\partial y}=0
$$

and

$$
\frac{U \partial U}{\partial x}+\frac{V \partial U}{\partial y}=v \frac{\partial^{2} U}{\partial y^{2}}
$$

We will introduce a stream function, $\psi$, given by,

$$
\begin{gathered}
\psi=U_{S} \sqrt{\frac{u x}{U_{S}}}\left\{\frac{U_{d}}{U_{S}} f(n)+n\right\} \\
\text { where } U_{S}=\frac{U_{1}+U_{2}}{2}, U_{d}=\frac{U_{1}-U_{2}}{2} \text { and }
\end{gathered}
$$

$n$, the similarity variable is given by, 


$$
n=y \cdot \frac{U_{S}}{v^{x}}
$$

$U_{S}$ is used in the definition of $n$ rather than $U_{d}$ in order that the square root will always be real for $x>0$ independent of the values of $U_{1}$ and $U_{2} \cdot$ (This assumes that one of the streams is not flowing in the negative $x$-direction). The components of velocity in the $x$ - and $y$-directions may now be written,

$$
\begin{aligned}
& U=\frac{\partial \psi}{\partial y}=U_{S}+U_{d} f^{\prime}(\eta) \text {, and } \\
& V=\frac{-\partial \psi}{\partial x}=\frac{U_{d}}{2} \sqrt{\frac{v}{x U_{S}}}\left\{n f^{\prime}(n)-f(n)\right\}
\end{aligned}
$$

The boundary conditions are

$$
\begin{array}{r}
U=U_{1} \text { as } \mathrm{y} \rightarrow \infty \\
\mathrm{U}=\mathrm{U}_{2} \text { as } \mathrm{y} \rightarrow-\infty \\
\text { and } U_{1} \mathrm{~V}_{1}+\mathrm{U}_{2} \mathrm{~V}_{2}=0 .
\end{array}
$$

In terms of the new similarity function the boundary conditions are,

$$
\begin{array}{ll}
f^{\prime}(n) \rightarrow I \text { as } n \rightarrow \infty & \text { ) } \\
f^{\prime}(n) \rightarrow-I \text { as } n \rightarrow-\infty & \{ \\
\left.U_{1}-U_{2}=\operatorname{Lin}_{n \rightarrow \infty}\left\{\frac{U_{1} f(n)+U_{2} f(n)}{n}\right\}\right) & \}
\end{array}
$$

It can be seen that our choice of the form (3.7) for the stream function leads to boundary conditions on the first derivative, which determines the mean velocity in the $x-$ direction which are independent of $U_{1}$ and $U_{2}$. Substituting equations (3.9) and (3.10) into equation 
(3.6) leads to the differential equation for $f$,

$$
2 f^{\prime \prime \prime}(n)+f^{\prime \prime}(n)\left[n+f(n) \frac{U_{d}}{U_{S}}\right]=0
$$

We see from equation (3.12) that $f(n)$ is not a universal function of $n$ for all values of $U_{d} / U_{s}$, thus we may write,

$$
f(n) \equiv f\left(n ; \frac{U_{d}}{U_{S}}\right)
$$

Equation (3.12) may be solved numerically subject to the boundary conditions (3.1.). As the method of solution is very similar to that used for the turbulent mixing problem we will deal with the numerical solutions for both cases after first deriving the appropriate equations for turbulent flow.

\subsubsection{The Turbulent Mixing of Two Parallel Streams}

In dealing with turbulent flow we may see that the effect of viscosity no longer controls the mean motion but affects the small scale motions involving dissipation and heat production. The essential difference, between laminar and turbulent flows, in the form of the momentum equations we use below, lies in the dominance of the Reynolds stress in comparison with the viscous terms. At present all solutions of the mean turbulent mixing problem require the use of an assumption for the nature of the shear stress. The two most widely used hypotheses, both introduced by PrandtI, are the mixing-length hypothesis, PrandtI (1925), and the constant exchange coefficient or constant eddy viscosity hypothesis, PrandtI (1942). Using the mixing length hypothesis, Tollmien (1926) 
obtained a solution for the mean velocity profile of a mixing layer with one stream at rest. An empirical constant related to the rate of spread of the layer had to be provided. Kuethe (1935) extended this analysis to the case of both streams having finite velocity. In his analysis he used the third boundary conation proposed by von Karman which we have earlier demonstrated is the appropriate one for incompressible flow. Gortler (1942) solved the same problem as Kuethe but used PrandtI's second hypothesis. Mills (1968) also made use of the eddy viscosity hypothesis in his numerical investigation of the mixing layer. He transformed the momentum and energy equations into equations for the shear stress and enthalpy with new independent variables $x$ and $U$. The equations for compressible flow were then solved by a method of successive approximation. A numerical solution has also been obtained by Baker and Weinstein (1968) for the problem of both homogeneous and heterogeneous mixing. We will now dexive the equation governing the mean motion in a turbulent mixing layer.

\subsubsection{Derivation of Equations}

Let us assume that two non-turbulent, parallel streams of velocity of $U_{1}$ and $U_{2}$ begin mixing at $x=0$. As in the case of laminar flow it is assumed that the flow is similar, at all stations downstream of $x=0$, for the mean flow in the mixing region.

Assuming that to the zeroth order pressure is constant everywhere inside the mixing region then for two-dimensionaI, incompressible flow the mean flow equations of continuity and 
and momentum may be written,

$$
\frac{\partial U}{\partial x}+\frac{\partial V}{\partial y}=0
$$

and

$$
\frac{U \partial U}{\partial x}+\frac{V \partial U}{\partial y}=\frac{v \partial^{2} U}{\partial y^{2}}-\frac{\partial \overline{u v}}{\partial y}-\frac{\partial}{\partial x}\left(\bar{u}^{2}+\vec{v}^{2}\right) .
$$

In the equations of motion of a turbulent fluid the viscous and normal stress terms are known to be small and they may be neglected. Then equation $(3.14)$ becomes,

$$
\frac{U \partial U}{\partial x}+\frac{V \partial U}{\partial y}=\frac{\partial \overline{u v}}{\partial y}
$$

Let us introduce the stream function $\psi$, given by,

$$
\psi=U_{S} L(x)\left\{\frac{U_{d}}{U_{S}} F(\xi)+\xi\right\}
$$

where $U_{S}$ and $U_{d}$ are defined as in the case of laminar mixing and $\xi$, the similarity variable is given by,

$$
\xi=\frac{y}{L(x)}
$$

where $L(x)$ is a measure of the mixing layer width to be defined more accurately later. We may now write the velocity components,

$$
U=U_{S}+U_{d} F^{\prime}(\xi)
$$

and $\quad \forall=U_{d} \frac{d t}{d x}\left\{\xi F^{\prime}(\xi)-F(\xi)\right\}$,

where primes denote differentiation with respect to $\xi$. Substituting equations (3.17) and (3.18) into equation (3.15) we obtain, 


$$
\frac{d L}{d x}\left\{\xi+\frac{U_{\bar{d}}}{U_{S}} F(\xi)\right\} F^{\prime} !(\xi)=\frac{d}{d \xi}\left(\frac{\overline{u v}}{U_{S} U_{d}}\right)
$$

It will be shown in Appendix I that we may use the third boundary condition given above, (3.2), for both the laminar and turbulent problems. Then the boundary conditions on $F(\xi)$ are,

$\left.\begin{array}{ll}F^{\prime}(\xi) \rightarrow 1 & \text { as } \xi \rightarrow+\infty \\ F^{\prime}(\xi) \rightarrow 1 & \text { as } \xi \rightarrow-\infty\end{array}\right\}$

The problem is to determine the mean fluctuating shear stress, $\overline{u v}$. The experiments of Wygnanski and Fiedler (1970) have shown that if measurements of the shear stress $\overline{u v}$, are made only when the measuring instrument is embedded in rotational flow, by means of conditional sampling, then the eddy viscosity, defined by $\nu_{T}=\left|\overline{u v} / \frac{\partial U}{\partial y}\right|$, is constant across the flow. RememberIng that we have considered the flow to be rotational throughout, it seems reasonable to assume that the eddy viscosity is given by,

$$
v_{\mathrm{T}}=\gamma \times \text { constant, where } \gamma \text { is the intermittency }
$$

function which is assumed to be a function of our similarity variable.

Let us therefore introduce an eddy viscosity defined by the relation,

$$
-\overline{u v}=v_{\mathrm{T}} \frac{\partial \mathrm{U}}{\partial \mathrm{y}}
$$

the substitution of (3.21) into equation (3.14) with only the normal stress terms omitted yields, 
$L \frac{d L_{i}}{d x}\left\{U_{S}+U_{d} F(\xi)\right\} F^{\prime \prime}(\xi)+\left(\nu+\nu_{T}\right) F^{\prime \prime}(\xi)+$

$\frac{\partial \nu_{T}}{\partial \xi} F^{\prime} \cdot(\xi)=0$

Let $\nu_{T}=v_{0} G(\xi)$ and $\sigma=\frac{\nu}{\nu_{0}}$, where $\nu_{0}$ is the maximum value of $v_{\text {IT }}$, then equation ( 3.22 ) may be written,

$\frac{U_{d}}{R_{T} \frac{d L}{d x}}\left\{\sigma F^{\prime \prime}+\frac{d\left(G \cdot F^{\prime}\right)}{d \xi}\right\}+\left\{U_{S} \xi+U_{d} F^{\prime} F^{\prime}=0\right.$

where $R_{T}$ is the turbulent Reynolds number defined by,

$$
R_{\mathrm{T}}=\frac{\left|\mathrm{U}_{\mathrm{d}}\right| \mathrm{L}}{\mathrm{v}_{\mathrm{O}}}
$$

The assumption of a constant eddy viscosity would lead to the equation,

$$
\frac{U_{d}(I+\sigma) F^{\prime \prime}}{U_{S} R_{T} \frac{d I}{d x}}+\left\{\xi+\frac{U_{d}}{U_{S}} F^{\prime}\right\} F^{\prime \prime}=0
$$

which is the laminar flow equation (3.12) with a change of coefficient on the third derivative of $F$. This fact was noted by Lin (1953) who was able to make use of Gortler's calculations for the turbulent mixing layer in his own calculations of the laminar flow problem.

\subsubsection{Solution of the Similarity Equations for Laminar and Turbulent Mixing Layers}

Before describing the method of solution which is employed for both the laminar and turbulent cases we will evaluate the coefficients in the turbulent similarity equation (3.23). We 
need to evaluate four factors,

i) the ratio of kinematic to eddy riscosities, $\sigma$,

ii) the distribution of eddy viscosity as a function of $\xi, G(\xi)$,

iij)the variation of turbulent Reynolds number with

velocity ratio, $R_{T T}\left(\frac{U_{d}}{U_{S}}\right)$,

and

iv) the variation of spread rate with velocity ratio.

The magnitude of the ratio of kinematic to eddy viscosity is very small. We can see from equation (3.23) that the ratio will only affect the solution appreciably in regions where $G(\xi)$ tends to zero. We will see later that this occurs at the edges of the mixing layer. The experimental results of Yule (1970) showed that the maximum value of eddy viscosity is given by,

$$
\frac{\nu_{0}}{x\left(U_{1}-U_{2}\right)}=7 \times 10^{-4}
$$

which result we will use in our calculations.

Lessen, Harpavat and Zien (1969) note that in their solution, choosing $G(\xi)=1$, the velocity profile is an error function and therefore,

$$
\frac{d U}{d \xi}=c_{1} \exp \left(-\frac{\xi^{2}}{2}\right)
$$

They axgue that as, from mixing length theory, the eddy viscosity is proportional to the magnitude of $\frac{\partial U}{\partial y}$, then a plausible distribution of eddy viscosity may be of the form, 


$$
v_{T}=\nu_{0} \exp \left(-A \xi^{2}\right)
$$

where $A$ is a constant with approximate value $\frac{1}{2}$. However, in our calculations the inflection point of the velocity profile does not occur at $\xi=0$ and thus a better representation of the eday viscosity distribution would be,

$$
\nu_{T}=\nu_{0} \exp \left[-A\left(\xi-\xi_{F}\right)^{2}\right]
$$

In our calculations the value of $\xi_{\mathrm{F}}$ is taken to be the value of $\xi$ for which $\frac{a^{3} F}{d \xi^{3}}=0$. This was calculated from a constant eddy viscosity analysis. When $\frac{d^{3} F}{d \xi^{3}}=0$ then $\frac{d U}{d y}$ is a maximum and from mixing-length arguments we may take this to be the position of the maximum in the eddy viscosity distribution. This may also be argued by considering single components of the fluctuating flow. Foote and tin (1950) considered the distribution of Reynolds' stress based on sclutions of the inviscid Orr-Sommerfeld equation. If $u$ and $v$ are given by,

and

$$
\begin{aligned}
& u=\hat{u}(y) e^{i \alpha(x-c t)} \\
& v=\hat{v}(y) e^{i \alpha(x-c t)}
\end{aligned}
$$

where $\hat{u}$ and $\hat{v}$ are the complex $y$-distributions of $u$ and $v$ satisfying the inviscid stability equation

$$
\hat{v}-\alpha^{2} \hat{v}+\frac{U+}{(\hat{U}-c)} \hat{v}=0,
$$

then $\overline{u v}$ is given by,

$$
\overline{u v}=\frac{1}{2 \alpha}\left(v_{i} v_{x}^{\prime}-v_{i}^{\prime} v_{r}\right) e^{2 \alpha c_{i} t},
$$


where the subscripts $i$ and $I$ refer to the imaginary and real parts respectively. Multiplying equation (3.29) by the complex conjugate of $\hat{v}$ we obtain,

$$
\hat{v}^{*} \hat{v}^{*}-\alpha^{2} \hat{v}^{*}+\frac{U^{\prime \prime}}{\left(U_{m} c\right)} v v^{*}=0
$$

The imaginary part of the equation is obtained by subtracting the complex conjugate of equation (3.31) from itself,

$$
\frac{d}{d y}\left(\hat{v}^{*} \hat{v}^{*}-\theta^{\prime * \vartheta}\right)=\frac{-i|\hat{v}|^{2} 2 c_{i} U^{\prime \prime}}{|U-c|^{2}}
$$

Now $\left(\hat{v}_{r} \hat{v}_{i}{ }^{\prime}-\hat{v}_{i} \hat{v}^{\prime}{ }^{\prime}\right)=-\frac{\dot{i}}{2}\left(\hat{v}^{\prime} \hat{v}^{*}-\hat{v}^{*} \hat{v}^{\prime}\right)$ and thus from equations $(3.32)$ and $(3.30)$ we have $\frac{\partial \tau}{\partial y}=\frac{-\rho}{2 \alpha} \cdot e^{2 \alpha c_{i} t} \cdot c_{i} \cdot \frac{U \cdot|\hat{v}|^{2}}{|U-c|^{2}}$,

where $\tau=-p \overline{u v}$ is the Reynolds' stress.

From equation (3.33) we see that the gradient of the Reynolds' stress is zero at the inflection point of the mean velocity profile. This simple example, which disregards the singularity $U=c$ which occurs in inviscid theory and also only considers temporal amplification theory (see Chapter 4), is given as a justification for the choice of the value of $\xi_{F}$ being the value of $\xi$ at which $\frac{\mathrm{d}^{3} \mathrm{~F}}{\mathrm{~d} \xi^{3}}$ is zero. At a later stage in this work we will discuss this form of representation of the disturbances in turbulent flow by particular single components of the disturbed flow. It will be seen that this method provids a good representation of the turbulence.

In our calculations we will consider three eddy viscosity distributions, 
i) constant eddy viscosity,

ii) equation $(3.27)$ and

iii) equation (3.28) where the value of $\xi_{F}$ is determined from the constant eddy viscosity analysis.

Iililey (1967) has suggested how the variation of turbulent Reynolas number with velocity ratio may be obtained. Let us Write the with of the mixing layex $L$,

$$
\mathrm{L}=\mathrm{W} \mathrm{X}
$$

where $w=W\left(\frac{U_{d}}{U_{S}}\right)$ is a function of the velocity ratio. Then substituting into equation (3.19) we obtain,

$$
\left\{\xi+\frac{U}{U_{S}} F^{\prime}(\xi)\right\} F^{\prime \prime}(\xi)=\frac{d}{d \xi}\left\{\frac{\overline{u V}}{U_{S} U_{d} W}\right\}
$$

We have previously noted that the similarity equation for turbulent Plow, equation (3.23), and that for laminar flow, equation (3.12), are of a similar form for the simple case of constant eddy viscosity. $\checkmark$ will also see that experimental results show that when mean velocity profiles of Iaminar and turbulent mixing regions are plotted against a variable based on the width of the mixing layer then the profiles coincide closely. Thus we could argue that for equation (3.34) to have a similar form, as a function of the velocity ratio, with the laminar case then $\overline{u v}$ has to be a function of $\xi$ and proportional to $\mathrm{U}_{\mathrm{S}} \mathrm{U}_{\mathrm{d}} \mathrm{W}$.

Thus from our definition of eddy viscosity we see that $\frac{\tau}{W_{p} U_{d}^{2}}=\frac{U T^{F^{\prime}}}{W L U_{d}}$, where $\tau$ is the Reynolds' stress defined above 
i.e. $\frac{\tau}{W_{\rho} U_{a}^{2}}=\frac{F^{\prime \prime}}{R_{T}^{W}}$, which may be written,

$$
\frac{\tau}{W_{S} U_{d}^{p}}=\frac{U_{d}}{U_{S} W} \cdot \frac{F^{\prime \prime}}{R_{T}}
$$

However, we require that $\frac{\tau}{\rho U_{S} U_{d} W}$ be independent of $\frac{U_{d}}{U_{S}}$ which implies from equation (3.35) that

$$
R_{T}=\text { const } x \frac{U_{d}}{U_{S} W}
$$

Gartshore (1965) has proposed that the turbulent Reynolds number is of the form

$$
\frac{1}{R_{T}}=\frac{I}{R_{T_{0}}}\left(1+\lambda\left|\frac{B}{A}\right|\right)
$$

where $A=\frac{\partial U}{\partial y}, \quad B=\frac{\partial U}{\partial x}$,

and $R_{T_{0}}$ is the value of the turbulent Reynolds number when $B=0$ which, in our case, occurs along the line $\xi=\xi_{0}$. The value of $\lambda$ is determined from experimental results. Newman (1967) obtains these constants and writes equation (3.37) in the form,

$$
\frac{1}{R_{T}}=.077\left\{1-9.1\left|\xi_{O}\right| W\right\}
$$

Substituting equation (3.36) into equation (3.38) we obtain,

$$
\frac{c U d}{U_{s} W}=\frac{1}{.077\left(1-9.1 / \xi_{0} / W\right.}
$$

and rearranging to obtain an equation for $W$ we have, 


$$
W=W\left(\frac{U_{d}}{U_{S}}\right)=\frac{\left(.077 \cdot \frac{U_{d}}{U_{s}}\right)}{\left(1+c_{1}\left|\xi_{0}\right| \frac{U_{d}}{U_{s}}\right)}
$$

Making use of this expression for W we obtain a reiationship for the turbulent Reynolds number as a function velocity ratio parametex $=\frac{U_{d}}{U_{s}}=\frac{U_{1}-U_{2}}{U_{1}+U_{2}}$,

$$
R_{T}\left(\frac{U_{d}}{U_{S}}\right)=R_{T}(I)\left\{\frac{I+c_{2}\left(\frac{U_{d}}{U_{S}}\right)}{I+c_{2}}\right\}
$$

From the numerical values given by Newman for $R_{T}$ for a iet with stizl surroundings and a small perturbation wake we may Write,

$$
R_{T}\left(\frac{U d}{U_{S}}\right)=32.9\left\{\frac{I+1.5\left(\frac{U_{d}}{U_{S}}\right)}{2.5}\right\}
$$

It ean be seen from equation (3.4I) and our definition of turbulent Reynolas number that the magnitude of the eddy viscosity decreases as the velocities of the two streams approach one another in order that there is a finite value of $\mathrm{R}_{\mathrm{r}}$ for this case.

Equation (3.39) gives the variation of spread rate with velocity ratio. We will now define the width paraneter $I(x)$ more accurately and then we will consider the analysis used by Abramovitch (1963) which will be compared with our present formulation.

We will define $L$ for the mixing layer as the distance between the points where the mean velocity differs from the average velocity of the two streams by $\frac{U_{d}}{2}$. This is shown 
in the diagram below
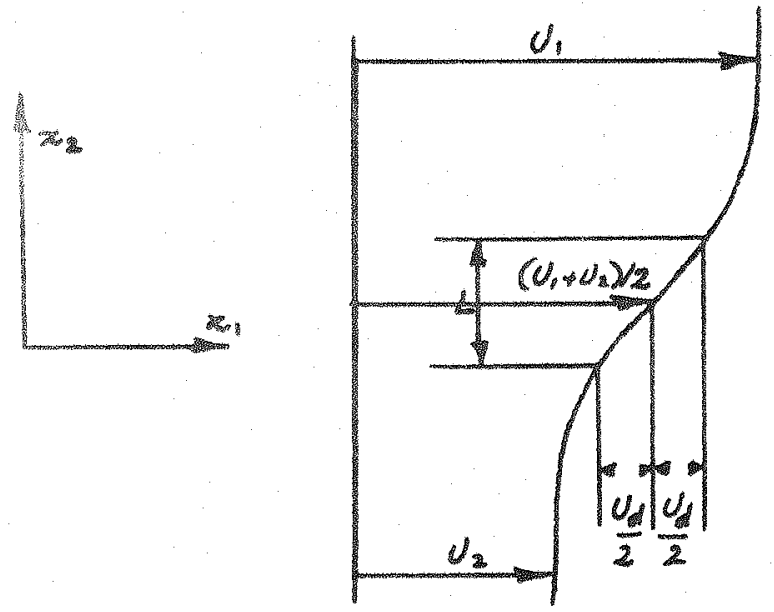

We will nowconsider the arguments used by Abramovitoh (1963) to describe the rate of spread of the mixing layer. He made use of mixing length ideas to conclude that the rate of incroase In the lajrex thickness. was proportional to the perturbation component of the transverse velocity which was proportional to the difference in velocities at the boundaries, i.e.

$$
\frac{d t}{d t} \propto v^{p}
$$

and

$$
v^{?} \propto U_{1}-U_{2}
$$

Making use of equation (3.42) with $\frac{d x}{d t}=U$ we obtain

$$
\frac{d I_{1}}{d x} \propto \frac{\left|U_{1}-U_{2}\right|}{|U|}
$$

We need t) determine the value of the mean characteristi: velocity in the denominator of equation (3.44). Abramovitch argues that for incompressible fluid this value is close to the axithmetic mean of the boundary velocities. Thus we 
may write,

$$
\frac{\partial I}{\partial x}=\psi\left[\frac{U_{1}-U_{2} \mid}{U_{1}+U_{2}}\right\}
$$

Newman (1967) expanded this as a polynomial,

$$
\frac{d I}{d x}=A_{0}+A_{1} \frac{\left|U_{1}-U_{2}\right|}{\left(U_{1}+U_{2}\right)}+A_{2} \frac{\left|U_{1}-U_{2}\right|^{2}}{\left(U_{1}+U_{2}\right)^{2}}+\cdots
$$

If $U_{1}-U_{2}=0$ then $\frac{d L}{d x}=0$ and thus $A_{0}=0$. Then taking the first term only in the expansion as chosen by Abramovitch we have,

$$
\frac{d I}{d x}=c \frac{\left|U_{1}-U_{2}\right|}{\left(U_{1}+U_{2}\right)}
$$

This result is the same as that obtained from our jurevicus analysis for the turbulent Reynolds number. From equation (3.39) we have,

$$
W=\frac{a I}{d x}=\frac{\left.k_{1} \frac{\left|U_{1}-U_{2}\right|}{U_{1}+U_{2}}\right)}{I+k_{2}\left(v_{1}+U_{2} \mid U_{2}\right)},
$$

where $k_{1}=0.077$ and $k_{2}=c_{1}\left|\xi_{0}\right|$. Expanding the denomirator we obtain,

$$
\frac{d \tau}{d x}=k_{1} \frac{\left|U_{1}-U_{2}\right|}{\left(U_{1}+U_{2}\right)}\left\{1-k_{2} \frac{U_{1}-U_{2}}{\left(U_{1}+U_{2}\right)}+\frac{k_{2}^{2}}{2} \frac{\left|U_{1}-U_{2}\right|^{2}}{\left(U_{1}+U_{2}\right)^{2}} \ldots\right\}
$$

The first term in the expansion (3.49) is identical. to the expression $(3.47)$. It may be expected that this first term representation will be valid for $U_{1}>U_{2}$. The variation 
of rate of spread with velocity ratio is shown in figurs 1. The collected results of Abramovitch (1963) show good agreement with the form of equation (3.47) though their numerical value is determined by the choice of $\mathrm{W}$ when $\mathrm{U}_{2}=0$. The results of Yule (1970) and Sabin (1965) suggest that the constant in shculd have a value of approximately.10. The constant given by Netman (1967) is .104. From equations $(3.39)$ and $(3.38)$ we may see that the choice of $c=k_{1}=.104$ leads to the result, $R_{T_{0}}=9.6$. The values of: $R_{\mathrm{T}}$ calculated from experiments is tabulatied by Newman, part of this table is shown below.

\begin{tabular}{|l|c|c|l|l|}
\hline Experimenter & Year & Range of $\mid \frac{U_{\text {defect }}}{U_{1}}$ & Type of Body & $R_{\mathrm{T}}$ \\
\hline Reichardt & 1951 & $.080-.057$ & $\begin{array}{l}\text { Circular } \\
\text { Cylinder }\end{array}$ & 10.4 \\
\hline Schlichting & 1960 & $.061-.025$ & $n$ & 13.0 \\
\hline Townsend & 1956 & $.045-.031$ & $n$ & 13.3 \\
\hline $\begin{array}{l}\text { Gartshore } \\
\text { Silverstein } \\
\text { et. al. }\end{array}$ & 1965 & $.111-.071$ & Square Cylinder & 8.6 \\
\hline
\end{tabular}

In his evaluation of the constants in equation (3.38), Newman uses experimental results and we may see from the above table that for the small perturbation wake $R_{T}$ Iies in the range $8.4 \rightarrow 13.3$. For the small perturbation wake, $\left|\frac{B}{A}\right| \rightarrow 0$ and thus $R_{T} \rightarrow R_{T}$. The average value of the experimental results above for $R_{T_{0}}$ is 10.7 which agrees more closely with the results obtained from the calculations from the rate of spread analysis. In order to achieve consistency in our calculations and also make use of the more easily measurable results, we will choose the constant 
$k_{3}=.204$. Then equations $(3.40)$ and $(3.47)$ become,

$$
\begin{aligned}
& R_{T}\left(\frac{U_{1}}{U_{3}}\right)=32.9\left\{\frac{1+1.43\left(\frac{U_{d}}{U_{S}}\right)}{2.43}\right\} \text { and } \\
& \frac{\partial I}{d x}=.104\left\{\frac{\left|U_{1}-U_{2}\right|}{\left(U_{1}+U_{2}\right)}\right\}
\end{aligned}
$$

These equations are used in our calculations for the turbulent mixing layer.

\section{4 .2 Numerical Method of Solution}

The same numerical procedure is used for the solution of both the laminax and turbulent problems. As the solution of the turbulent case, equation (3.23) is algebraically more complicated, we will examine it in detail and only give the corresponding equations for laminar flow when convenient.

Equation (3.23) may be written,

$$
\left\{\xi+\frac{U_{d}}{U_{S}}\left(F+\frac{1}{R_{T} \frac{d L}{d x}} G^{\prime}\right)\right\} F^{\prime \prime}+\frac{\frac{U_{d}}{U_{S}}}{R_{T} \frac{d I}{d x}}\{\sigma+G\} F^{\prime \prime}=0
$$

Let us introduce a new function $P(\xi)$ defined by,

$$
P(\xi)=\frac{d F(\xi)}{d \xi},
$$

then equation $(3.52)$ becomes,

$$
\left\{\xi+\frac{d}{U_{s}}\left(F+\frac{I}{P_{T} \frac{d I}{d x}} G^{\prime}\right)\right\} P^{\prime}+\left\{\frac{\frac{d}{U_{S}}}{R_{T} \frac{d T}{d x}}(\sigma+G)\right\} P^{\prime \prime}=0
$$

The equivalent form of equation (3.12) for laminar flow is,

$$
\left[n+\frac{U_{d}}{U_{S}} f p^{\prime}+2 p^{\prime \prime}=0\right.
$$




$$
\text { where } F(n)=\frac{a f(n)}{d n}
$$

We will row write these equations in finite difference form. Equation (3.53) may be written

$$
\left(F_{x}-F_{x-1}\right)=\frac{1}{2} h\left(P_{x}+P_{r-1}\right)
$$

where $h=s$ the step difference in $\xi$ between stations ' $r-I$ ' and ' $x$. Equation (3.54) may be written,

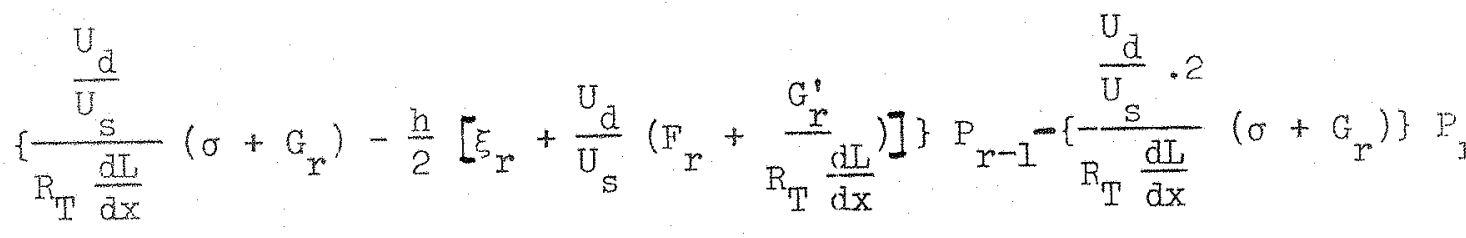

$$
\begin{aligned}
& +\left\{\frac{U_{T}}{R_{T} \frac{d I}{d x}}\left(o+G_{r}\right)+\frac{h}{2}\left[\xi_{r}+\frac{U_{d}}{U_{S}}\left(F_{r}+\frac{G_{r}^{\prime}}{R_{T} \frac{d I}{d x}}\right)\right]\right\} P_{r+1}=0
\end{aligned}
$$

The equivalent finite difference form of equation $(3.55)$ is,

$$
\left\{4-h\left(n_{x}+\frac{U_{d}}{U_{s}} f_{r}\right)\right\} p_{r-1}-8 p_{r}+\left\{4+h\left(n_{r}+\frac{U_{d}}{U_{s}} f_{r}\right)\right\} p_{r+1}=0
$$

Equations (3.58) and (3.59) are solved by an iterative procedure. An initial assumption is made for the values of $F^{\prime}(i)$. In our calculations a sine form was chosen. The value of $r(\xi)$ is then calculated using equation (3.57). The finite difference equation (3.58) may be written in matrix form,

$$
|A| \bar{P}=\bar{B}
$$

where $|A|$ is a triple diagonal matrix and $\bar{P}$ and $\bar{B}$ are column vectors, the first and last elements of $\bar{B}$ being determinad by 
the boundiary conditions on $P(\xi)$ and the rest of its elements being zero. The system of linear algebraic equations $(3.60)$ is solved using Gaussian elimination without pivots giving : ncw value for $P(\xi)$. The new value of $F(\xi)$ is calculated by evalua Hing the fransverse velocity according to equation (3.18) and applying the third boundary condition (3.2). The iteration procedure is repeated until successive values of $P(\xi)$ differ by less than a chosen small quantity.

\subsubsection{The Computer Program}

The flow diagram of the program is show in figure $\therefore$. The data to be read in are the velocities of the two streams, the ratio of kinematic to eddy viscosity, the step size and the numerical value of the desired accuracy of the iteration. Having evaluated $U_{d} / U_{S}$ the subroutines SPREAD and RTURB are called which evaluate $\frac{\mathrm{d}}{\mathrm{dx}}$ and $\mathrm{R}_{\mathrm{T}}$ according to equations (3.50) and (3.1) respectively. The subroutine EDIVIS then calculates the distribution of eddy viscosity for either constant eddy viscosty or according to equations (3.27) or equation (3.28), where $F$ is previously determined from a calculation involving constent eddy viscosity. The initial guess at the values of $P(\xi)$ is calculated as a sine function and the values of $F(\xi)$ are calculatea from equation (3.57). The set of linear algekraic equations are calculated and solved using Gaussian IPlimiration without pivots. The new value of $P(\xi)$ is then taken as the average value of $\mathrm{P}_{I}$ and $\mathrm{P}_{I+I}$. If convergence is not achjeved within ter iterations sets of four iterated values are ured 
to calculste the new value of $P(\xi)$ using inear intexpolstion. When the sum of the differences $P_{I+1}-P_{I}$ becomes Iess tran the desired value, the program outputs the values of $F^{\prime}(\xi), V(\xi)$ and their derivatives.

\subsection{Computation Results}

The calculations were performed for values of $\mathrm{U}_{2} / \mathrm{U}_{1}$ of 0.61 and 0.0 . The rixst value of velocity ratio was chosen so that comparison coula be made with the measurements of Yule (1970). Results from the calculations using the second velocity xatio were required for our subsequent calculations of finite amplitude effects on the growth of the mixing layer.

The computed function $f^{\prime}(n)$ for laminar flow and velocity ratio 0.61 is shown in Figure 3. The calculations are compared with those of Iin (1953) for a velocity ratio of 0.667 . Lin's results have been recalculated for a change in definition of the similarity variable, n.

The convergence of the iteration scheme is shown in figure 4. It was Pound that as the value of $\mathrm{U}_{2}$ tended to zero so the convergence of the solution became slower. If a simple iteration scheme is used where $P_{I+I}=P_{I}$ as many as 800 iterations were required. The iterative scheme used in the calculations for figure 4 set $P_{I+1}=\frac{P_{I}+P_{I+1}}{2}$ for the first ten calculitions and then sets of four iterated values of $P_{I}$ were used in a Inear interpolation for the new value of $P$.

The effect on the velocity distribution of the choice of eddy viscosity distribution is shown in figure 5. Here the 
function F" $(\xi)$ is presented, where

$$
F *(\xi)=\frac{1}{U} \frac{d^{2} U}{d \xi^{2}}
$$

It can be seen that is the physically incorrect assumption of a symatrac eddy viscosity distribution is considered then the maximun absolute magnitude of second derivative of velouty occurs towards the lower velocity edge of the shear layer. If the constant eddy viscosity assumption is made then the magnitude of the second derivative of velocity decreases and the solution tends towards the laminar result as can be seen from examination of equation $(3.24 a)$.

The effect of choice of the constant $A$ in equation (3.27) is shown in Figure 6 . The variation is most noticeable at the edges of the mixing layer. The experimental results of Yule (1970) are plotted for comparison. It twas found that a value of $A$ of 0.7 gave best agreement with these expeximental results. We have rreviously noted that the distribution of eddy riscosity given by $G(\xi)$ should correspond to the intermittenay of the flow. In figure 7 the experimental values of intermittency measured by Wygnanski and Fiedler (1970) are compared wi.th $G(\xi)$ with $A=0.7$. The agreement is very good, especially in the centre of the region. The experiments by Wygnanski and Fiedler do however suggest that the value of $A$ in our definition of eddy viscosity should take two values, one for the high velocity side of the layer and another for the lower velocity side. 
It is interesting to note that the shear stres is an integral function of the product of the $z$-component of the vorticity disturbance and the $y$-direction velocity fluctuation. This was noted by Lilley (1967) who put,

$$
\overline{\frac{\partial u z}{\partial y}}=\overline{\omega_{3} v}
$$

Integrating equation (3.6I) with respect to $y$ we obtain

$$
\overline{u v}=\int_{-\infty}^{y} \overline{v \omega_{3}} d y^{\prime}
$$

Making use of mixing length ideas we would expect the integrand in equation (3.62) to have a positive peak at $y=-y_{1}$ and a negative peak at $y=y_{2}$, where $y_{1}$ and $y_{2}$ lie close to the points of maximum value of $\frac{\partial^{2} U}{\partial y^{2}}$. This is sketched below. The corresponding integral is also sketched and we see that we have predicted a constant shear stress across the flow
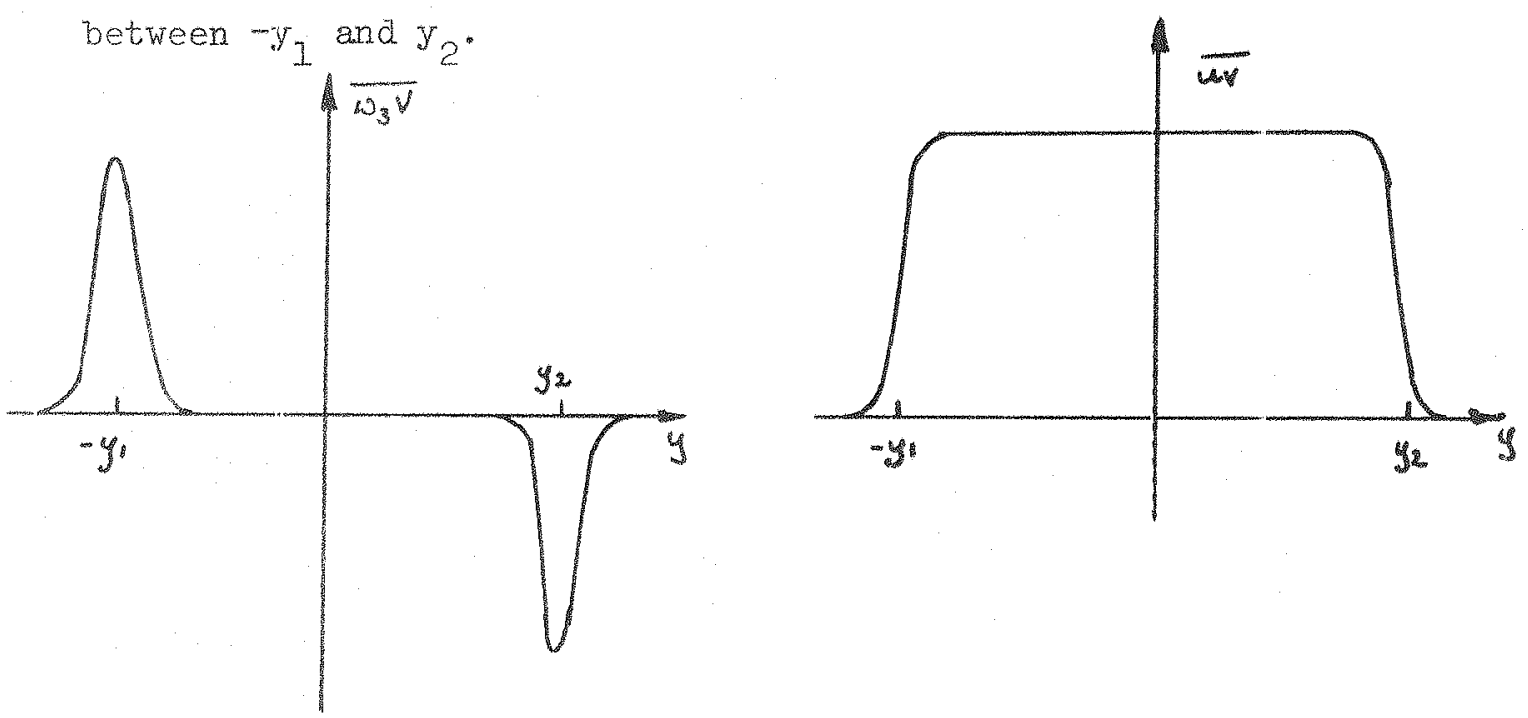

Howerer, we have previously noted that we have assuraed that 
the rlow is rotational throughout whereas the above analysis refers orly to the turbulent fluid. Thus we would expert that taking account of intermittency would result in a rounding off of the wy distribution. From equation (3.21) we have trat the eddy viscosity is given by, $\nu_{T}=-\overline{u v} / \frac{\partial U}{\partial y}$, and so we woulc expect that the distribution of eddy viscosity in equation (3.28) would be too lerge outside the values $y=-y_{1}$ and $y=y_{2}$. Thuse two positions are shown on Figure 7 and we note that our prediction does appear to be too large outside these values.

The function $F^{\prime}(\xi)$ for turbulent flow and velocity ratio 0.61 is shown in pigure 8. The constants for the calculation were,

(i) $A=0.7$

(ii) $\sigma=.0033$

and

(iij) $\xi_{\mathrm{F}}=-.13123$

The experimental results of Yule are plotted for comparison and the agreement is good over the whole mixing laver. The constant eddy viscosity calculation is also shown for comparison and it can be seen that the agreement between theory and experiment is poor at the edges of the mixing layer.

By considering the momentum integral equation (2.23) we may define the momentum thickness of the mixing layer between streasns of velocity $U_{1}$ and $U_{2}$ as, (

$$
\theta=\frac{3}{U_{a}^{2}} \int_{-\infty}^{\infty}\left(U_{1}-U\right)\left(U-U_{2}\right) d y
$$


Then for the laminar flow case we may write,

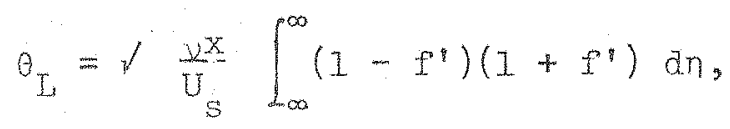

and for the turbulent case,

$$
\theta_{T}=L \int_{-\infty}^{\infty}\left(1-F^{\prime}\right)\left(1+F^{\prime}\right) d \xi
$$

Thus the non-dimensional co-ordinate $y / \theta$, is giren, in the Laminar case, by

$$
y / \theta_{I}=n /\left[\int_{-\infty}^{\infty}\left(1-f^{\prime}\right)\left(1+f^{\prime}\right) d \eta\right]
$$

and in the turbulent case by,

$$
y / \theta_{T}=\varepsilon /\left[\int_{-\infty}^{\infty}\left(1-F^{2}\right)\left(1+F^{8}\right) d \xi\right]
$$

Making use of equations (3.66) and (3.67) the functions $F^{\prime}$ and $f^{\prime}$ are plotted as functions of $\left(\frac{\mathrm{y}-\mathrm{b}}{\theta}\right)$ in figure 9 , where $b$ is the value of $y$ at which $U=U_{S^{*}}$ As Sato (1956) poirts out, in his work on the transition of the mixing layer, the two curves show so slight $a$ difference that it is difficult to tell the transition point from the mean profiles alone. The calculations in figure 9 are for a value of $U_{2}=0$. The results are compared in the laminar case with the calculations of Iin and in the turbulent case with the experiments of tiepmann and Laufer (1947). The agreement is good except for the turbulent case at the lowest velocity eage of the mixing region where measurements of the mean velocity are most difficult. 
The measurements in this region are not reliable as the axial velocity is no longer large in comparison with the transverse velocity. Liepmann and Laufer note that their hotwire measurements are accurate in the range $\infty>\theta>-0.8$. They compared their hot-wire measurements with the measurements using a total head tube and the results differed outside the range given above. In this region the hot-wire will produce an output which is crudely proportional to the square root of the sums of the squares of the axial and transverse velocities. Thus the recorded value of axial velocity will be greater than its true value, which agrees with our theoretical prediction. At a later stage we will use this non-dimensional form of the mean velocity profile to represent the shape of the mean velocity profile through transition.

The calculated results for the two velocity ratios are tobulated in Tables I and II.

In the next chapter we will consider the analytic and numerical methods for the solution of the Orr-Sommerfeld. Equation, which will be subsequently solved using the mean velocity profiles calculated in this chapter. 
CHAPTER 4

Review of Methods of Solution of the Orr-Sommerfela Equation

In chapter 2 we derived the Iinear equation of hydrodynamic stability for parallel mean flow ind taree dimensionat disturbances. Before we go on to solve this equation for the case of the stability of a laminar mixing layer, and at a later stage solve a similar equation in our discussion of the turbulent mixing layer, it is thought to be useful to review previous analytic and numerical methods of solution of the Orrm-Somerfeld equation.

The Orr-Sommerfeld equation is an ordinary linear differential equation of the fourth order. The methods of analysis used in its solution have been variational methods (Lee and Reynolds, 1964), expansion methods (Dolph and Lewis, 1958) and asymptotic methods. Because of the magnitude of the Reyrnolds number, the asymptotic methods have played the most important role in analytical solutions. With the advent of the high speed digital computer, numerical methods have recently proved very successful. A collection of the various asymptotic methods is presented by $\operatorname{Lin}$ (1955) and more recently accounts of developments in the methods of solution have been presented by Chandrarasekbar (1961) and Betchov and Criminale (1967).

The stability equation derived in Chapter 2, equation (2.21a), is rewritten now for convenience,

$$
\begin{aligned}
& \left(U-\frac{\omega}{U}\right)\left(\hat{v}^{\prime \prime}-\left(\alpha^{2}+\beta^{2}\right) \hat{v}\right)-U^{\prime \prime} \hat{v}+\frac{i}{\alpha R}\left(\hat{v} \hat{v}-2\left(\alpha^{2}+\beta^{2}\right) \hat{v}^{\prime \prime}+\right. \\
& \left.\left(\alpha^{2}+\beta^{2}\right)^{2} v\right)=0
\end{aligned}
$$


We will now introduce a set of transformations, used by Squire (1933), that reduces equation (4.1) for the threedimensional disturbance to an equivalent two-dimensional problem. Let us set,

$$
\begin{array}{ll}
v=\hat{v}, \quad c=\omega / \alpha, \quad \alpha^{2}=\alpha^{2}+\beta^{2}, & \text { ) } \\
\overline{\alpha u}=\alpha \hat{u}+\beta \hat{w} \text { and } \overline{\alpha R}=\alpha R & \text { ) }
\end{array}
$$

Equation (4.1) then becomes,

$$
(U-c)\left(v^{\prime \prime}-\bar{\alpha}^{2} v\right)-U^{\prime \prime} v+\frac{i}{\overline{\alpha R}}\left(v^{i v}-2 \bar{\alpha}^{2} v^{\prime \prime}+\bar{\alpha}^{-4} v\right)=0
$$

Equation (4.3) is the Orr-Sommerfeld equation for a two-dimensional disturbance with wave-number $\bar{\alpha}$ in the $x$-direction and Reynolds number $\bar{R}$. It can be seen from (4.2) that $\bar{R}<R$. Thus we may confine our discussions of hydrodynamic stability to twodimensional disturbances noting that results for a three-dimensional disturbance may be derived from the two-dimensional results.

\subsubsection{Analytical Methods}

The earliest analyses in hydrodynamic stability theory were concerned with the inviscid problem. As it is expected that instability in the mean flow profile will occur at high Reynolds rumber it is interesting to examine the inviscid form of equation (4.3) which is obtained as $\bar{R} \rightarrow \infty$,

$$
(U-c)\left(v^{\prime \prime}-\alpha^{2} v\right)-U^{\prime \prime} v=0
$$

where the bars denoting transformed terms have been dropped as we are confining ourselves to a discussion of two-dimensional disturbances. Equation (4.4) is known as the Inviscid Orr- 
Sommerfeld or Rayleigh equation. Lord Rayleigh (1880) showed that an inflection point in the mean velocity profile was a necessary condition for flow to be unstable. This result may be easily derived. Let us multiply equation (4.4) b.y $v^{*}$, the complex conjugate of $\mathrm{V}$, divide by $(U-c)$ and integrate between some limits $y_{1}$ and $y_{2}$. We then obtain,

$$
\int_{y_{1}}^{y_{2}}\left[\left(v^{*} v^{*}\right)^{\prime}-v^{\prime} v^{*}-a^{2} v v^{*}\right] d y-\int_{y_{1}}^{y_{2}} \frac{U^{\prime \prime} v v^{*}}{(U-c)} d y=0
$$

The first term may be integrated exactly as follows,

$$
\int_{y_{1}}^{y_{2}}\left(v^{\prime} v^{*}\right) \cdot d y=\left[v^{\prime} v^{*}\right]_{y_{1}}^{y_{2}}
$$

The limits $y_{1}$ and $y_{2}$ are either located at walls or at \pm+ and in either case the integral term, equation (4.6), will vanish. It then follows that we may write equation (4.5),

$$
\int_{y_{1}}^{y_{2}} \frac{u^{\prime \prime} v v^{*}}{(u-c)} d y=-k^{2}
$$

where $\mathrm{k}$ is a real number and $\alpha$ is assumed real. For complex $c$ the imaginary part of equation (4.7) is

$$
c_{i} \int_{y_{1}}^{y_{2}} \frac{u^{\prime \prime}}{v v^{*}} \frac{\left(U-c_{r}\right)^{2}+c_{i}^{2}}{d y}=0
$$

On examining this equation we see that unless $c_{i}$ is zero then $U^{\prime \prime}$ must have at least one zero in the range of integration.

A further result derived by H申iland (1953) demonstrated that an unstable oscillation can only exist if the mean vorticity has an absolute maximum within the flow at the point of inflection. 
Howerer, it must be noted that these results are for inviscid flow and thus it is possible for a mean velocity profile with no inflection point to be unstable if the viscous component; of the Orr-Sommerfeld equation are retained.

The Orr-Sommerfeld equation will have four linearly independent solutions and we may write the solution,

$$
v=\sum_{n=1}^{4} k_{n} v_{n}
$$

where $V_{n}$ are the independent solutions and $k_{n}$ are four constants determined from the boundary conditions which may be written,

$$
v_{1}=v_{1}^{\prime}=v_{2}=v_{2}^{\prime}=0,
$$

where the suffices 1,2 refer to the position of the boundary conditions. In the case of free shear flows the boundary conditions are applied at infinity and in bounded flows are applied at the walls. For a boundary layer one pair of conditions is ajplied at the wall, the other pair at infinity. For a non-trivial solution,

$$
\left|\begin{array}{cccc}
V_{11} & v_{21} & v_{31} & v_{41} \\
V_{1.2} & v_{22} & v_{32} & v_{42} \\
V_{11}^{\prime} & V_{21}^{\prime} & V_{31}^{\prime} & v_{41}^{\prime} \\
V_{12}^{\prime} & V_{22}^{\prime} & V_{32}^{\prime} & v_{42}^{\prime}
\end{array}\right|=0
$$

This relationship may be written,

$$
F(c, R, c)=0
$$


Equation (4.12) is a secular equation formed from the eigenvalue problem posed by equations $(4.3)$ and $(4 \cdot 10)$. We see from equation (4.12) that the parameters affecting the ware propagation are the Reynolds number $R$, the wavenumber $\alpha$, vihich may be real or complex and $c=c_{r}+i c_{i}$. In the solution of the Orr-Sommerfeld equation two cases arise. The first will be referred to as temporal amplification and is characterized by the following properties,

$\alpha$ is real, $\quad \omega=\alpha c$ is complex, $c_{r}$ is the phase velocity, $c_{i}<0$ corresponds to damped disturbances, $c_{i}=0$ corresponds to neutral disturbances and $c_{i}>0$ corresponds to amplified disturbances.

The second case will be referred to as spatial amplification and is similarly characterized by,

$\omega=\alpha c$ is real, $\alpha$ is complex $\alpha_{i}<0$ corresponds to amplified disturbances, $\alpha_{i}=0$ corresponds to neutral disturbances and $\alpha_{i}>0$ corresponds to damped disturbances.

Until recently only the temporal amplification was studied but experixnental investigations and the works of Gaster (1963, 1965a, 1965b) have now focussed attention on the spatial problem. However the secular equation (4.12) applies to both cases. We will now examine a number of analytical approaches to the solution of the Orr-Sommerfeld equation. 


\subsubsection{The Asymptotic Solutions of the Orr-Sommerfeld Equation}

A description of various methods of solution is given by Reid (1965) and we will summarise his work here.

We wi.ll first examine solutions at iarge value:s of aR by an expansion of the solution in inverse powers of $i \alpha R$,

$$
v(y)=v^{(0)}(y)+\frac{1}{(i \alpha R)} v^{(1)}(y)+\cdots
$$

where the first term in the expansion, $v^{(0)}(y)$ satisfies the inviscid equation (4.4), which is singular at the point or points where $(U-c)$ is zero unless $U^{\prime \prime}$ also vanishes there. Tollmien (1929) obtained the two solutions as a power series in $y-y_{c}$, where $y_{c}$ is the value of $y$ at which $U=c$. In the neighbourhood of the singular point there exists the analytic solution,

$$
v_{A}(y)=\left(y-y_{c}\right) P_{A}\left(y-y_{c}\right),
$$

where

$$
P_{A}\left(y-y_{c}\right)=1+\frac{U_{c}^{\prime \prime}}{2 U_{c}^{\prime \prime}}\left(y-y_{c}\right)+\left(U_{c}^{\prime \prime}+\alpha^{2}\right)\left(y-y_{c}\right)^{2}+\ldots
$$

The second linearly independent solution is of the form,

$$
\begin{aligned}
& \left.\mathrm{V}_{B}(y)=P_{B}\left(y-y_{c}\right)+\frac{U_{c}^{\prime \prime}}{U_{c}^{\prime}}\left(y-y_{c}\right) P_{A}\left(y-y_{c}\right) \log \left(y-y_{c}\right)\right) \\
& \left.P_{B}\left(y-y_{c}\right)=1+\left(\frac{U_{c}^{\prime \prime \prime}}{2 U_{c}^{\prime}}-\frac{U_{c}^{\prime \prime}}{U_{c}^{\prime 2}}+\frac{1}{2} \alpha^{2}\right)\left(y-y_{c}\right)^{2}+\ldots\right)
\end{aligned}
$$

where

In the solution of complete Orr-Sommerfeld equation:s Heisenberg (1924), made use of the WKB method. We will derive these solutions as follows; let

$$
v(y)=\exp \left\{{ }^{j} g(y) d y\right\},
$$


then $g$ satisfies the non-Iinear third order equation,

$$
\begin{aligned}
& g^{4}+6 g^{2} g^{\prime}+4 g g^{\prime \prime}+3 g^{\prime 2}+g^{\prime \prime \prime}-2 \alpha^{2}\left(g^{2}+g^{\prime}\right)+\alpha^{4} \\
& =\operatorname{i\alpha R}\left\{(U-c)\left(g^{2}+g^{\prime}-\alpha^{2}\right)-U^{\prime \prime}\right\}
\end{aligned}
$$

We will assume an expansion for $g$ of the form,

$$
g(y)=(i \alpha R)^{\frac{1}{2}} g_{0}(y)+g_{1}(y)+(i \alpha R)^{-\frac{1}{2}} g_{2}(y)+\ldots
$$

Substitution into equation (4.17) and equating the coefficients of like powers of $(i \alpha R)^{\frac{1}{2}}$ to zero, leads to

$$
\begin{aligned}
g_{0}^{4} & =(U-c) g_{0}^{2} \\
4 g_{0}^{3} g_{1}+6 g_{0}{ }^{2} g_{0}^{\prime} & =(u-c)\left(g_{0}^{\prime}+2 g_{0} g_{I}\right)
\end{aligned}
$$

which admits solutions,

$$
g_{0}= \pm(U-c)^{\frac{1}{2}}, g_{I}=-\frac{5}{4} \frac{U^{\prime}}{(U-c)} \cdots
$$

Retaining the first two terms only of (4.18) we obtain,

and

$$
\begin{aligned}
& \left.V_{3}(y)=\frac{1}{2} \pi^{-\frac{1}{2}}(U-c)^{-5 / 4} \exp \left\{-(\alpha R)^{\frac{1}{2}} q(y)\right\}\right) \\
& \left.V_{4}(y)=\frac{1}{2} \pi^{-\frac{1}{2}}(U-c)^{-5 / 4} \exp \left\{+(\alpha R)^{\frac{1}{2}} q(y)\right\}\right)
\end{aligned}
$$

where

$$
q(y)=\int_{y_{c}}^{y}\{i(u-c)\}^{\frac{1}{2}} d y .
$$

The choice of the path of integration of the integral is the subject of much analysis and a detailed discussion is given by Wasow (1948). The solutions $(4.21)$ are not valid near the critical point or if the critical point lies close to a boundary, that is, 
if $\left|y_{1}-y_{c}\right|$ becomos as $O(\alpha R)^{-1 / 3}$.

It is interesting to note that if $\left|y-y_{c}\right|$ is small compared with unity but large compared with $(\alpha R)^{-1 / 3}$ then $(4.21)$ is simplified. to

$V_{3,4}(y) \sim \frac{1}{2} \pi^{-\frac{1}{2}}\left\{U_{c}^{\prime}\left(y-y_{c}\right)\right\}^{-5 / 4} \exp \left\{ \pm \frac{2}{3}\left(i \alpha R U_{c}^{\prime}\right)^{\frac{1}{2}}\left(y-y_{c}\right)^{3 / 2}\right\} \quad(4.22)$

It will be seen that this limiting solution agrees with that obtained in subsequent analysis.

In order to obtain approximations to $\mathrm{V}_{3}$ and $\mathrm{V}_{4}$ that are valid in the neighbourhood of a critical point we will introduce a stretched independent variable of the form,

$$
\xi=\left(y-y_{c}\right)\left(i \alpha R U_{c}^{\prime}\right)^{1 / 3},
$$

and a new dependent variable,

$$
x^{(0)}(\xi)=v(y)
$$

If we substitute these expressions into the Orr-Sommerfeld equation with only the dominant terms for small values of $\left|y-y_{c}\right|$ retained, that is

$$
v^{i v}=i \alpha R U_{c}^{\prime}\left(y-y_{c}\right) v^{\prime \prime}
$$

then we obtain,

$$
\left(\frac{d^{2}}{d \xi^{2}}-\xi\right) \frac{d 2}{d \xi^{2}} x^{(0)}=0
$$

Equation (4.26) has four solutions which we may write in the form,

$$
x_{1}^{(0)}=\left(y-y_{c}\right), x_{2}^{(0)}=1, x_{3}^{(0)} \text { and } x_{4}^{(0)} \text {. }
$$


The first two solutions are identifiable with the leading terms in the inviscid solutions $v_{A}$ and $v_{B}$, equations (4.14) and (4.15) The two viscous solutions are given by Iin (1955) as

$$
\begin{aligned}
& \left.\left.x_{3}^{(0)}=\int_{\infty}^{n} \int_{\infty}^{n} n^{\frac{1}{2}} H_{1 / 3}(1) \cdot \frac{2}{3}(i n)^{3 / 2}\right] d n d n\right) \\
& \text { and }) \\
& \left.x_{4}^{(0)}=\int_{-\infty}^{n} \int_{-\infty}^{n} n^{\frac{1}{2}} H_{1 / 3}(2)\left[\frac{2}{3}(i n)^{3 / 2}\right] \text { a } n d n\right)
\end{aligned}
$$

Where $n=\left(y-y_{c}\right)\left(U_{c}^{\prime} \alpha R\right)^{I / 3}=-\xi$

Reid notes that in attempting to study flows with two critical points it is advantageous to use the Airy function rotation. Then the viscous solutions may be written,

$$
\begin{array}{ll}
\left.x_{3}{ }^{(0)}(\xi)=\left(\varepsilon U_{c}^{\prime}\right)^{-5 / 4} \iint A_{i}(\xi) d \xi d \xi\right) & j) \\
\text { and } & \\
\left.x_{4}{ }^{(0)}(\xi)=\left(\varepsilon U_{c}^{\prime}\right)^{-5 / 4} \iint P_{2}(\xi) d \xi d \xi\right)
\end{array}
$$

where $\varepsilon=\left(i \alpha R U_{c}^{\prime}\right)^{-1 / 3}$ and $P_{2}(\xi)=A_{i}\left(\xi_{0} e^{2 \pi i / 3}\right)$ and the limits of integration are determined by analysis similar to that required in the WKB solutions. The limiting solutions, for $\left|y-y_{c}\right|$ are small compared with unity but Jarge compared with $|\varepsilon|$, are

$$
x_{3,4}^{(0)}(\xi) \sim \frac{1}{2} \pi^{-\frac{1}{2}}\left(\varepsilon U_{c}^{\prime}\right)^{-5 / 4} \xi^{-5 / 4} \exp \left( \pm \frac{2}{3} \xi^{3 / 2}\right)
$$

which is identical to the limiting form of the WKB solutions. This demonstrates that there exists an overlapping range of validity of the WKB and Airy function solutions defined by the conditions $|\varepsilon| \ll\left|y-y_{c}\right| \ll 1$. 
Holstein (1950) tabulated the function $x_{3}{ }^{(0)}$ which he denoted by $\phi_{3 I}(n)$ and is given in our notation by,

$$
\phi_{31}(n)=-2 \sqrt{3} e^{\frac{-\pi i}{12}} \iint A_{i}(\xi) d \xi d \xi
$$

The method of matched asymptotic expansions was used by Graebel (1966) to determine the viscous solutions. He expanded $\mathrm{v}(\mathrm{y})$ in the inner and outer regions according to the series,

$$
\begin{aligned}
& \left.v(y, \mu) \sim \sum_{n=0}^{\infty} \varepsilon_{n}^{*}(\mu) \quad v_{n}^{*}(n) \text { for the inner region and }\right) \\
& v(y, \mu) \sim \sum_{n=0}^{\infty} \bar{\varepsilon}_{n}(\mu) \bar{v}_{n}(n) \text { for the outer region, where } \\
& \mu n=y-y_{c} \text { and } \mu=o(\alpha R)^{-n} \text {. Lins method gives } \mu=(\alpha R)^{-1 / 3} .
\end{aligned}
$$

The coefficients in ( 4.31 ) are made to satisfy,

$$
\lim _{\mu \rightarrow 0}\left(\frac{\varepsilon_{n}^{*}+1}{\varepsilon_{n}^{*}}\right)=\operatorname{lin}_{\mu \rightarrow 0}\left(\frac{\bar{\varepsilon}_{n}+1}{\bar{\varepsilon}_{n}}\right)=0
$$

The coefficients $\varepsilon_{n}^{*}$ and $\bar{\varepsilon}_{n}$ are determined by a matching procedure.

Eagles (1969) defined certain exact solutions of the Orr-Sommerfeld equation by using well known convergent series. Asymptotic approximations to the exact solutions were then obtained in the form of a composite series by using the matching procedure of Van Dyke (1964) modified by Fraenkel (1969). He found he required no separate expansion near the wall as employed by Graebel. Eagles also discussed the Lin-Heisenberg approximation to the eigenvalue relation. He concluded that 
this was correct in general trend but needed to be treated with caution if accuracy is important. However, he noted tha' for the case of Poiseuille flow very accurate results for the neutral curve could be obtained using this approximation.

As we have seen, in the above discussion of asymptotic solutions, there are regions of inapplicability to separate methods. Also, even when an analytic form of solution has been obtained, a numerical calculation of the functions involved is required. Thus it is now more attractive to attempt a direct numerical solution of the Orr-Sommerfeld equation making use of high-speed digital computers. So we will now discuss the various numerical methods that have been developed for the solution of the Orr-Sommerfeld equation.

\subsection{Numerical Methods}

The numerical methods of solution may be broadly divided into two groups. The first method involves the finite djeference form of the Orr-Sommerfeld equation which leads to a set of linear algebraic equations. The second method involves the use of a stepping procedure using direct numerical integration.

Neariy all developments in numerical methods have been initially applied to flow problems with either one or two boundaries. The difference between these calculations and those for free shear flows lies only in the application of the boundary conditions. Thus we will examine numerical procedures for various flow problems noting that in most cases these methods may be applied to free shear flows. 
The numerical solution of Thomas (1953) was based on finite disference equations and applied to Poiseville flow. The finite difference for the stability equation leads to a set of simultaneous linear algebraic equations which may be written in matrix form as,

$$
\bar{A} \bar{X}=\lambda \bar{B} \bar{X},
$$

where $\bar{A}$ and $\bar{B}$ are coefficient matrices, $\bar{X}$ is the eigenvector and $\lambda$ the eigenvalue. The dimensions of the matrices depend on the number of steps into which the range of integration is divided. Thomas used 50 steps and had thus to solve a 50 × 50 matrix to obtain the eigenvalues and eigenfunctions. As we will discuss further the accuracy of the computations involving matrix methods depends on the accuracy with which a matrix may be inverted and is limited to low values of $\alpha$ and $R$. Thomas's method was applied by Kurtz and Crandall (1962) to the Blasius profile and the free convection boundary layer on a vertical plate. Gallagher and Mercer (1962) solved the Couette flow problem employing the Galerkin method. They reported that difficulties were found in their calculations when the value of $\alpha R$ exceeded 1000 .

A variational method for investigating the stability of parallel flows has been developed by Lee and Reynolds (1964, 1967) and applied to various bounded and unbounded f'low problems. They first defined the differential operator,

$$
L[V]=\left[\left(D^{2}-\alpha^{2}\right)^{2}-i \alpha R \quad\left\{(U-c)\left(D^{2}-\alpha^{2}\right)-D^{2} U\right\}\right] v=0(4.34)
$$

where $D$ is the differential operator $\frac{d}{d y}$. The boundary 
conditions are

$$
v=D V=O \text { at the two boundaries }
$$

In order to construc, the adjoint problem, $(4.34)$, is muliplied by $v^{*}$, the adjointfunction, then integrated from one boundary to onother, i.e.

$$
I=\int_{\mathrm{y}_{I}}^{\mathrm{y}_{2}} \mathrm{v}^{*} \mathrm{~L}[\mathrm{~V}] d \mathrm{y}
$$

Integrating (4.36) by parts we have,

$$
\begin{aligned}
& I=\left[v * D^{3} v-D v^{*} D^{2} v+D^{2} v * D v-D^{3} v * v-2 \alpha^{2} v * D v+2 \alpha^{2} D v^{*} v\right) \\
& \left.-i \alpha R^{\prime}\left\{U v^{*} D v-U D v^{*} v-D U v^{*} v-c v^{*} D v+c D v^{*} v\right\}\right]^{y_{2}} \\
& \mathrm{y}_{1} \\
& +\int_{y_{1}}^{y_{2}} v\left[D^{4} v^{*}-2 \alpha^{2} D^{2} v^{*}+\alpha^{4} v^{*}-i \alpha R\left\{U D^{2} v^{*}-c D^{2} v^{*}-\alpha^{2}(U-c) v^{*}\right)\right.
\end{aligned}
$$

If the boundary conditions for the adjoint problem are chosen to be the same as the given problem,

$$
V^{*}=D v^{*}=0 \text { at the boundaries, }
$$

then (4.37) may be rewritten in the form,

$$
I=\int_{y_{1}}^{y_{2}} v L^{*}\left[v^{*}\right] d y,
$$

from which we may write the adjoint differential operator and equation as,

$$
L^{*}\left[V^{*}\right]=\left[\left(D^{2}-\alpha^{2}\right)^{2}-i \alpha R^{\prime}\left\{(U-C)\left(D^{2}-\alpha^{2}\right)+2 D U D\right\}\right] v^{*}=0 \quad(4.39)
$$


Lee and Reynolds characterised the variational formulation of the problem by the requirement that the integral

$$
I=\int_{y_{1}}^{y_{2}} v^{*} L[V] d y,
$$

is stationary under first order variations in the functions $\mathrm{v}$ and $\mathrm{v}^{*}$ where $\mathrm{v}$ and $\mathrm{v}^{*}$ are solutions to their respective problems. They then used the Ritz method to reduce the problem to a set of algebraic equations as follows. Let the functions $\mathrm{V}$ and $\mathrm{V}^{*}$ be expanded as

$$
\begin{array}{ll}
v(y)=\sum_{j=1}^{k} a_{j} f_{j}(y) \quad & \{ \\
\left.v^{*}(y)=\sum_{i=1}^{k} a_{i}^{*} f_{i}^{*}(y) \quad\right\}
\end{array}
$$

where $a_{j}$ and $a_{i}^{*}$ are complex coefficients and $f_{j}$ and $f_{i}^{*}$ are carefuly chosen functions that fit the boundary conditions. Since the boundary conditions are identical the same approximoting functions may be taken for each set. Substituting (4.40) into $(4.36)$ gives

$$
I=\sum_{i=1}^{k} \sum_{j=1}^{k} a_{i}^{*} a_{j} \int_{y_{1}}^{y} f_{i} L\left[f_{j}\right] d y
$$

Since $I$ is to be stationary

$$
\frac{\partial I}{\partial a_{i}^{*}}=0 \quad i=1, k
$$

and so we obtain from (4.41) a set of algebraic equations

$$
\sum_{j=1}^{k} a_{j} \int_{y_{I}}^{y_{2}} f_{i} L\left[f_{j}\right] d y=0
$$


which has non-trivial solutions if

$$
\operatorname{Det}\left[\int_{y_{1}}^{y_{2}} f_{i} L\left[f_{j}\right] d y\right]=0
$$

Tee and Reynolds wrote the operator $I$ in the form $I=M-\lambda N$ then the secular equation (4.43) becomes

$$
\begin{array}{ll} 
& \operatorname{Det}\left[A_{i j}-\lambda B_{i j}\right]=0 \\
\text { where } & A_{i j}=\int_{y_{I}}^{y_{f_{i}}} M\left[f_{j}\right] d y \\
\text { and } & B_{i j}=\int_{y_{I}}^{y_{2}} f_{i} N\left[f_{j}\right] d y
\end{array}
$$

Fquation (4.44) was then solved for the eigenvalues $\lambda$. The functions $f_{i}$ chosen by Lee and Reynolds for various fllows were

(i) Poiseuille flow, $\left.f_{n}(y)=\left(1-y^{2}\right)^{2} y^{2(n-1)}\right)$

(ii) Couette flow, $\left.\quad f_{n}(y)=\left(1-y^{2}\right)^{2} y^{n-1}\right)$

(iii)Plane Jet Flow, $\quad f_{n}(y)=\operatorname{sech}^{n}(\alpha y)$

Lee and Reynolds' results showed good agreement with other methods of calculation and the convergence of the eigenvalue calculations was fast.

As has been mentioned, the accuracy of methods involving the solution of matrix equations depends on the accuracy with which a matrix may be inverted. When the matrix is illconditioned the task can be very long and so we will now examine numerical methods that use a step-by-step integration procedure.

A scheme for a step-by-step integration method was suggested by Brown (1959), for the Blasius profile. He noted that 
when $y>\delta$, where $\delta$ is the value of $y$ when the free stream values are reached within a specified amount, then the solution of the Orr-Sommerfeld may be written exactly as,

$$
v=c_{1} e^{\alpha y}+c_{2} e^{-\alpha y}+c_{3} e^{\beta y}+c_{4} e^{-\beta y}
$$

where $\beta=\beta_{r}+i \beta_{i}$ and

$\beta_{x}=\frac{1}{\sqrt{2}}\left[\alpha^{2}+\alpha c_{i} R+\left\{\left(\alpha^{2}+\alpha c_{i} R\right)^{2}+(\alpha R)^{2}\left(U_{\delta}-c_{x}\right)^{2}\right\}^{\frac{1}{2}}\right]$

$\left.\beta_{i}= \pm \frac{1}{\sqrt{2}}\left[-\left(\alpha^{2}+\alpha c_{i} R\right)+\left\{\left(\alpha^{2}+\alpha c_{i} R\right)^{2}+(\alpha R)^{2}\left(U_{\delta}-c_{x}\right)^{2}\right\}^{\frac{1}{2}}\right]^{\frac{1}{2}},\right\}(4.47)$

the sign of $\beta_{i}$ being the same as the sign of $\left(U_{\delta}-c_{x}\right)$.

In order to satisfy the boundary conditions at infinity $c_{1}=c_{3}$

$=0$. Thus

$$
v=c_{2} e^{-a y}+c_{4} e^{-\beta y}
$$

If the subscript 2 denotes values at $\mathrm{y}=\delta$ then the function $\mathrm{v}$ and its derivatives yield two equations,

$$
\begin{aligned}
& v_{2}^{\prime \prime}+\alpha v_{2} \prime-\beta^{2}\left(v_{2}^{\prime}+\alpha v_{2}\right)=0 \text { and } \\
& v_{2}^{\prime \prime \prime}+\beta v_{2}^{\prime \prime-\alpha} \alpha^{2}\left(v_{2}^{\prime}+\beta v_{2}\right)=0
\end{aligned}
$$

for $y<\delta$ the solution can be written

$$
v=k_{1} v_{1}+k_{2} v_{2}+k_{3} v_{3}+k_{4} v_{4}
$$

If the $v^{2} s$ are defined as follows:

$$
\begin{aligned}
& v_{1}(0)=1+i, v_{1}^{\prime}(0)=v_{1}^{\prime \prime}(0)=v_{1}^{\prime \prime \prime}(0)=0, \\
& v_{2}(0)=0, v_{2}^{\prime}(0)=1+i, v_{2}^{\prime \prime}(0)=v_{2}^{\prime \prime \prime}(0)=0,
\end{aligned}
$$




$$
\begin{aligned}
& v_{3}(0)=v_{3}^{\prime}(0)=0, v_{3}^{\prime \prime}(0)=1+i, v_{3}^{\prime \prime \prime}=0 \quad \text { and } \\
& v_{4}(0)=v_{4}^{\prime}(0)=v_{4}^{\prime \prime}(0)=0, v_{4}^{\prime \prime \prime}=1+i
\end{aligned}
$$

then insertion of th boundary conditions at the wall,

$$
\begin{aligned}
& v=v^{\prime}=0 \quad \text { when } y=0, \quad \text { leads to, } \\
& 0=k_{1}(1+i) \\
& 0=k_{2}(1+i), \text { so that the complete solution can be expressed }
\end{aligned}
$$
as:

$$
v=k_{3} v_{3}+k_{4} v_{4}
$$

At the boundary layer edge the two solutions (4.48) and (4.50) must coincide. The method suggested by Brown first sets $k_{3}=1$. The functions $v_{3}$ and $v_{4}$ are then integrated from $y=0$ to $y=\delta$ and this gives the values at $y=\delta, i . e \cdot v_{32} v_{32}^{\prime}$ etc. Substitution of equation (4.50) into either equation (4.49) will detexmine $k_{4}$. The other equation (4.49) has then to be satisfied by adjusting the parameters $\alpha, R$ and $c$. Brown found that the digital computers available to him were not accurate to a large enough number of digits and were too slow for accurate enough step-sizes. However, the method developed by Brown of applying boundary conditions, e.g. equation (4.49), corresponds to the method we will use in our own numerical computations. The reason for Brown's difficulty in the numerical integration can be seen readily if we examine the behaviour of the solutions in a simple flow case. Let us consider the problem where,

$$
U=I, U^{n}=0 \text {. }
$$


then the solutions of the Orr-Sommerfeld equation, which correspond to equations $(4.46)$ and $(4.47)$ are,

$$
\begin{aligned}
& V_{I, 2} \sim \exp [j \mathrm{y}] \\
& V_{3,4} \sim \exp \left[ \pm\left\{\alpha^{2}+i \alpha R(I-c)\right\}^{\frac{1}{2}} y\right]
\end{aligned}
$$

The boundary conditions only permit the solutions with negative exponents for $y>0$, i.e. $V_{1}$ and $V_{3}$. Examination of the form of (4.52) shows that the $V_{3}$ solution depends on the Reynolds number and will grow rapidly in comparison with the $V_{I}$ solution which is dependent on $\alpha$. Thus the parasitic exror from the rapidiy growing $V_{3}$ solution will soon dominate the $V_{I}$ solution if integration is performed. We therefore need a numerical method to inhibit this contamination.

A scheme developed by Kaplan (1964) provided the first attempt at preventing the contamination of the $V_{1}$ solution. His procedure divided the range of integration into a number of segments and then the solution was purified at the start of each segment. Kaplan's method and its modification by Betchov and Cximinale (1967) is summarised here.

The solution of the Orr-Sommerfeld equation is written in the form,

$$
v(y)=\sum_{j=1}^{4} A_{j} e^{p_{j} Y}
$$

where, at any thin slice of the boundary layer, for large Reynolds number 


$$
\begin{aligned}
& \left.p_{1}=\left(\alpha^{2}+\frac{U^{n}}{U-c}\right)^{\frac{1}{2}} \quad, \quad p_{2} \simeq-\left(\alpha^{2}+\frac{U^{n}}{(U-c)}\right)^{\frac{1}{2}}\right) \\
& \left.p_{3}=(\alpha R(U-c))^{\frac{1}{2}} \quad, \quad p_{4} \simeq-(\operatorname{iaR}(U-c))^{\frac{1}{2}}\right)
\end{aligned}
$$

Prysically $A_{1}$ and $A_{2}$ represent inviscid rotational oscillations and $A_{3}$ and $A_{4}$ correspond to viscous vorticity waves.

The numerical solution is started by integrating across the boundary layer to obtain a solution containing as much as possible of the $A_{4}$ mode. This is performed by setting $A_{1}=$ $A_{2}=A_{3}=0$ and $A_{4}=1$ at $y=1$ and integrating across the entire region storing the values of $v$ and its derivatives at each step. Let us denote this function as $(v)_{\delta 0^{*}}$ The computation is then started again at $y=1$ with $A_{z}=1$ and $A_{1}=A_{3}=$ $A_{4}=0$. Integration proceeds to a station $y_{1}$. The computer then integrates across the region $\mathrm{y}_{1}$ to $\mathrm{y}_{2}$ etc. If the solution from $y=1$ to $y_{1}$ is denoted by $s_{1}$ and that from $y_{1}$ to $y_{2}$ by $s_{2}$ then we define $S_{2}$ as follows,

$$
S_{2}=S_{1}+a_{1} S_{0}
$$

where $S_{0}$ is the solution obtained on the first complete pass. The new function $v$ is then related to the two others by

$$
(v)_{S_{2}}=(v)_{S_{1}}+a_{1}(v)_{S_{0}}
$$

The coefficient $a_{I}$ is determined by the condition that the vorticity mode $A_{4}$ of solution $S_{2}$ must vanish at $y=y_{1}$. The lis 
means that

$$
a_{1}=-\frac{\left(A_{4}\right) S_{I}}{\left(A_{4}\right)_{S_{0}}}
$$

Kaplan assumed that e" ept near the critical layer and very close to the wall the eigenfunction satisfies the Rayleigh equation and he thus put

$$
A_{4}=(U-c)\left(v^{\prime}+\alpha^{2} v\right)-U^{3} v .
$$

Betchov and Criminale obtained $A_{4}$ from relation (4.53) and its derivatives. Neglecting $A_{1}$ and $A_{2}$ the relations for $V^{\prime \prime}$ and iv yield,

$$
A_{4}=\frac{v^{i v}-p_{3} v^{i n}}{2\left(p_{3}\right)^{3}} e^{p_{3}^{y}}
$$

The initial values for $S_{2}$ at $y=y_{1}$ may now be obtained by applying equation (4.56) at $\mathrm{y}=\mathrm{y}_{1}$. The computation then proceeds to a new station $y_{3}$ and the decontamination procedure is repeated. When the wall is reached the final solution will be of the form,

$$
S_{W}=S_{I}+\left(a_{I}+a_{2}+\cdots a_{W-I}\right) S_{O}
$$

At the wall the zero normal velocity condition gives

$$
v(0)=(v)_{S W}(0)+A(v)_{S_{0}}(0)=0
$$

The zero tangential velocity condition with equation (4.61) gives

$$
V^{\prime}(0)=(v)_{S_{W}}(0)-\frac{(v)_{S W}(0)}{(v)_{S_{O}}(0)}(v) S_{S_{0}}(0)=0
$$

This equation provides the eigenvalue criterion and the value of 
$v^{\prime}(0)$ is adjusted to be zero by altering the values of the parameters $\alpha, R$ and $c$.

This rethod has jeen used by Wazzan, Okamura and Smith (1966) in a modified : rm using a scaling factor when storing the rapidy growing solution. The method is also used by Hussain and Reynolds (1970) in their study of a perturbation wave in turbulent shear flow. However, the method is criticised by Wazzar, Okcmura and Smith (1967) because of its Iimited range of application: up to $R=10^{5}$. Both Sharma (1968) and Davey and Nguyen (1971) were unable to obtain accurate results using this method, the former author considering the boundary layer and the latter, pipe flow. In both these papers and that of Wazan et al. (1967) the authors used the much more elegant technique of orthogonalisation which we will use in our computations. A detailed description of this technique will be given at a later stage.

\subsection{Spatial and TemporaI Amplification}

In our discussion of methods of solution of the Orr-Sommerfeld equation, we have confined ourselves to the problem of temporal amplification. We have defined this model as having real Reynolds number and wave-number and a complex value of $c$, the real part of $c$ being the phase velocity and the imaginary part the amplification ractor.

The experimental investigation of Schubauer and Skramstad. (1943) was originally accompanied by theory considering temporal amplification. However, the waves observed experimentally grew spatially with distance from the vibrating ribbon and a trans- 
formation had to be used which gave the spatial growth rate apparent to an observer moving at a speed $c_{r}$ where $c_{r}$ is given by,

$$
c_{x}=\frac{u}{\alpha}: \text {, the phase velocity, }
$$

Where a is the real wave number. Gaster (1965a) observes that the type of waves generated by Schubauer and Skramstad should have a complex wavenumber and real frequency, $w$, defined by

$$
\omega=a_{c}
$$

However the correspondence between experimental results analysed using the transformation (4.63) agreed well with the theory. The relationship between temporal and spatial param meters has been examined by Gaster (1965b, 1962).

The characteristic equation (4.12) may be written in the form,

$$
F(\alpha, \omega)=0,
$$

for a particular velocity profile and Reynolds, where $\omega=\alpha c=$ $\omega_{r}+i \omega_{i}$ is the angular frequency, $\alpha=\alpha_{r}+i \alpha_{i}$ is the complex wavenumber and $c=c_{x}+i c_{i}$. For every value of $w$ a value of $\alpha_{3} \alpha_{x}+i \alpha_{i}$, may be found so that one may map lines of constant $\alpha_{r}$ and $\alpha_{i}$ on the wolane. Gaster (1965a) notes that the two families of curves are orthogonal except at points where there is a singularity in the solution of $(4.65)$. It will be assumed that no such singularity occurs in the region of interest near the real axis. 
It will be assumed that $\omega$ is an analytic function of $\alpha$. The two cases of interest are the spatially increasing modes, With $\omega$ real, and the temporally growing modes with $\alpha$ real. These modes thus occur on $t_{\text {. }}$ lines $\omega_{i}=0$ and $\alpha_{i}=0$ respectively. Along the line $\omega_{i}=0$ we have,

$$
\omega_{i}=\alpha_{i} c_{r}+\alpha_{r} c_{i}=0
$$

With our assumption of an analytical relationship between $\omega$ and $\alpha$ we may write,

$$
\omega=\omega r\left(\alpha_{r}, \alpha_{i}\right)+i \omega_{i}\left(\alpha_{r}, \alpha_{i}\right)
$$

and the Cauchymiemann relations hold,

$$
\frac{\partial \omega_{r}}{\partial \alpha_{r}}=\frac{\partial \omega_{i}}{\partial \alpha_{i}}
$$

and

$$
\frac{\partial \omega_{r}}{\partial \alpha_{i}}=-\frac{\partial \omega_{i}}{\partial \alpha_{r}}
$$

Now let us integrate these relations from a point on the temporal curve, $\alpha_{i}=0$, to a point on the spatial curve, $w_{i}=0$, along a line of constant $\alpha_{r} i . e_{\text {. }}$

$$
w_{i_{T}}=-\int_{0}^{\alpha_{i_{S}}} \frac{\partial \omega_{r}}{\partial \alpha_{r}} d \alpha_{i}
$$

and $\omega_{r_{S}}-\omega_{r_{r}}=-\int_{0}^{\alpha_{i_{S}}} \frac{\partial \omega_{i}}{\partial \alpha_{r}} d \alpha_{1}$

where the subscripts $T$ and $S$ denote values on the temporal and spatial curves respectively. Gaster argues that the integrand in equation (4.70) will be of the order of the maximum value 
of $\omega_{3}$ for a given Reynolds number and thus the integral term as whole will be very small and we may write,

$$
\omega_{r_{S}}=w_{r_{T}}
$$

Expanding $\frac{\partial \omega_{r}}{\partial \alpha_{r}}$ about a point $\alpha_{i}^{*}$ on the const. $\alpha_{r}$ line and substitution in (4.69) leads finally to,

$$
\frac{\omega_{i_{T}}}{\alpha_{i_{S}}}=-\frac{\partial \omega_{r}}{\partial \alpha_{r}}\left(\alpha_{i}^{*}\right)+0\left\{\alpha_{i_{S}} \cdot w_{i_{\max }}\right\} \text { and to the same }
$$

accuracy as $(4.71)$ we have,

$$
\frac{w_{i_{r p}}}{\alpha_{i_{b}}}=-\frac{\partial w_{r}}{\partial \alpha_{r}}\left(\alpha_{i}^{*}\right)
$$

where $\frac{\partial w_{r}}{\partial \alpha_{r}}$ may be evaluated at any point on the $\alpha_{r}=$ const curve between the temporal and spatial curves. Thus we see that for small amplification factors the real parts of $\omega$ and the real parts of $\alpha$ calculated from temporal and spatial considerations are equal. From (4.72) we see that the spatial growth rate is equal to the temporal growth rate divided by a factor known as the group velocity, that is

$$
a_{i_{S}}=-w_{i_{T}} / c_{g}
$$

where $c_{g}$ is the group velocity. Now from (4.63) we see that the spatial amplification may not be calculated from the termporal amplification in this manner unless,

$$
\frac{\partial \omega_{r}}{\partial \alpha_{r}}=\frac{\omega_{r}}{\alpha_{r}}
$$


and the group velocity then equals the phase velocity. In the boundary layer where waves may be considered non-dispersive, this relationship will be approximately true, which accounts for the apparent suc. ssful comparison of theory and experiment by Schubauer and Skramstad.

Although most previous theoretical investigations have considered temporal amplification the use of numerical techniques enables investigations of spatial amplification directly with no great increase in difficulty。

In this chapter we have examined the development of Iinearised stability theory which has now reached a stage where comparison with experiments in a number of different flows has produced convincing agreement. However, this theory based on the assumption of infinitessimal disturbances, predicts a very rapid growth rate for unstable modes and so non-linearity and finite amplitude disturbances need to be examined. A review of non-Iinear effects is given at a later stage when calculations for finite amplitude disturbances in a mixing layer are presented. 


\section{CHAPTER 5}

The Stability of Mixing Layer between Iwo Streams

In this chapter we will describe the numexical method to solve the Orrm-Somerfeld equation. Calculations will then be made of the stability of the mixing layer between two streams of finite velocity using the mean velocity profile calculated in Chapter 3. The effect of divergence of the flow will be discussed and corrections made to the stability eigenvalues.

\subsection{Numerical Method of Solution}

The Orr-Sommerfeld equation of hydrodynamic stability is written,

$$
\begin{aligned}
& (u-c)\left\{u_{2}-\alpha^{2} \hat{u}_{2}\right\}-u u_{2}+\frac{i}{\alpha R}\left\{\hat{u}_{2} i v-2 \alpha^{2} \hat{u}_{2}\right. \\
& \left.+\alpha \hat{u}_{2}\right\}=0
\end{aligned}
$$

and the boundary conditions are

$$
\dot{u}_{2}=\dot{u}_{2}=0 \text { as } x_{2} \rightarrow+\infty \text {, }
$$

Where the primes denote differentiation with respect to $\mathrm{x}_{2}$ " In equation (5.1) the parameters that are determined by the mean flow are $U$, the mean velocity profile, $U^{\prime \prime}$, its second derivative with respect to $x_{2}$, and the Reynolds number $R$ given by,

$$
R=\frac{U_{d}^{L}}{v}
$$

In order to more readily use the form of mean velocity 
profile derived in Chaptex 3 we will define the mean velocity as,

$$
U\left(n^{*}\right)=r+u_{d} f^{\prime}\left(n^{*}\right)
$$

where $n^{*}=x_{2} / \theta$ and the momentum thickness $\theta$ is defined by

$$
\theta=\frac{1}{u^{2}} \int_{-\infty}^{\infty}\left(U_{1}-U\right)\left(U-U_{2}\right) d x_{2}
$$

Substitution of expression (5.4) into equation (5.1) leads to,

$$
\begin{aligned}
\left(f^{*}\left(n^{*}\right)-c^{*}\right)\left\{\hat{u}_{2}+\alpha^{*} \hat{u}_{2}\right\}-f^{\prime \prime}\left(n^{*}\right) \hat{u}_{2}+\frac{i}{\alpha^{*} R_{\theta}} \\
\\
\left\{\hat{u}_{2}{ }^{j v}-2 \alpha^{2} \hat{u}_{2}^{\prime \prime}+\alpha^{*} \hat{u}_{2}\right\}=0,
\end{aligned}
$$

Where $\alpha^{*}=\alpha \theta, R_{\theta}=\frac{U^{\theta}}{v}$ and $-c^{*}=\left[\frac{U_{s}}{U_{d}}-c\right]$

primes denote differentiation with respect to $\eta^{*}$. It can be seen that equations $(5.1)$ and $(5.6)$ are identical in form, however in equation (5.6) the wavenumber and Reynolds number are functions of $x$.

Above the mixing layer $f^{\prime}\left(\eta^{*}\right)$ attains a constant value $I$, and $f^{*}\left(n^{*}\right)$ is zero. In this region the solution to equation (5.6) may be written,

$$
\dot{u}_{2}=\sum_{n=1}^{4} \dot{u}_{2}=\sum_{n=1}^{4} A_{n} e^{p_{n} n^{*}},
$$

$$
\left.\begin{array}{cc}
\text { where } \quad p_{1}=\alpha^{*}, \quad p_{2}=-\alpha^{*}, \\
\left.p_{3}=\left[\alpha^{*}+i \alpha^{*} R_{\theta}\left(1-c^{*}\right)\right]^{\frac{1}{2}} \text { and } p_{4}=-\left[\alpha * \alpha^{2}+i \alpha^{*} R_{\theta}\left(1-c^{*}\right)\right]^{\frac{1}{2}}\right)^{(5.8)}
\end{array}\right\}
$$


Below the mixing layer $f^{\prime}\left(n^{*}\right)$ attains a constant value -1 and $f^{*}\left(n^{*}\right)$ is zero. In this region the solution to equation (5.6) may be written.

$$
\dot{u}_{2}=\sum_{n=1}^{4} \dot{u}_{2}=\sum_{n=1}^{4} B_{n} e^{q_{n} n^{*}},
$$

$$
\begin{gathered}
\text { where } \left.q_{1}=\alpha * \alpha_{2}^{*}=-\alpha^{*}\right) \\
\left.q_{3}=\left[\alpha^{*}-i \alpha^{*} R_{\theta}(1+c *)\right]^{\frac{1}{2}} \text { and } q_{4}=-\left[\alpha *^{2}-i \alpha^{*} R_{\theta}(1+c)\right]^{\frac{1}{2}}\right)
\end{gathered}
$$

In the solutions above the mixing layer we see that $A_{1}=A_{3}=0$ in order that the boundary conditions on $\hat{u}_{2}$ may be fulfilled. Below the mixing layer we also have $B_{2}=B_{4}=0$ 。

In the above solutions we see that above the mixing layer $v_{z_{2}}=A_{2} e^{p_{2} n^{*}}$ corresponds to the 'inviscid solution and $\hat{u}_{2_{4}}^{2}=A_{4} e^{p_{4} n^{*}}$ involves the Reynolds number and corresponds to the "viscous solution". Similarly, below the mixing layer, the solution $u_{2_{1}}=B_{1} e^{q_{1} n^{*}}$ corresponds to the "inviscid solution" and $\hat{u}_{2}=B_{3} \quad e^{q_{3} n^{*}}$ is the "viscous solution'. Let us consider the solutions for $n^{*}>0$ as we will numerically integrate from above to below the mixing layer.

It can be seen from the form of $\hat{u}_{2_{2}}$ and $\hat{u}_{2_{4}}$ that the viscous solution will vary rapialy with $n^{*}$ depending on the value of the Reynolds number, whilst the inviscid solution will be well-behaved. In any numerical integration procedure with starting conditions corresponding to,

$$
A_{2} e^{-\alpha \eta^{*}} \text { and } A_{4} e^{P_{4} n^{*}}
$$


the parasitic error in the numerical technique will inevitably produce a viscous $\mathrm{sc}$ tion, The problem is to obtain th ir viscid solution with ut any viscous influences.

Kaplan (1967) found that accurate results were possible if the inviscid solution is made free of a part of the parasitic error due to the viscous solution by satisfying the Rayleigh equation at each step of the integration. This method does reduce the error in the inviscid solution and its first deriv ative, however as the Rayleigh equation is of second order the higher derivatives are not as well corrected. The method does not work at high Reynolds numbers and, due to the filtering system being the inviscid stability equation, the method also fails in regions of dominant viscous effects such as critical layers and wall layers. As we have discussed in Chapter 4 for problems where the critical layers are far from the walls the Kaplan purification scheme has to be abandoned. In our calculations, in order to remove restrictions on the Reynolds number and wavenumbers, a different purification scheme is used throughout. The method which we will use to prevent the contamination of the inviscid solution is based on preserving the linear independence of the solutions.

The set of solutions (5.7) are fundamentally linearly independent functions. In numerical integration we have noted that the rapidly varying viscous solution affects the inviscid solution and eventually dominates it, that is the solutions are no longer linearly independent. The method we 
will adopt to preserve the Iinear independence of the solutions is to obtain an orthonormal system of vectors corresponding to the Iinearly indeper i at solutions.

The formation of a set of orthogonal vectors from a system of vectors is a well-known method in vector analysis. Let us consider the two Inearly independent vectors $A$ and $B$. We Wish to construct a vector orthogonal to $A$ of the form $\underset{\sim}{B}-\mathrm{kA}$ where Is is a scalar variable. Thus we may write

$$
\stackrel{A}{A} \cdot\left(B-\frac{k A}{n}\right)=0
$$

Rearranging this expression we may see that the scalar variable $k$ is formed by the dot product of the vector $\underset{\sim}{B}$ and the normam Iised vector As that is,

$$
k=\frac{A}{|A|^{2}} \cdot B,
$$

Where $|A|$ denotes the absolute squared value of the vector $A$. From the definition of Iinear independence the vector $(B-k, A)$ cannot vanish. Thus we have constructed a pair of orthogonal vectors from our original pair of linearly independent vectors. The adaptation of this procedure to the solution of differential equations was made by Bellman and Kalaba (1965). Let us write the inviscid solution $\vec{u}_{2}$ as the vector $\forall_{2}$ where $y_{2}$ is given by

$$
v_{2}=\left(\hat{u}_{2}, \hat{u}_{2}, \hat{u}_{2}, \hat{u}_{2}, \hat{u}_{2},\right)^{\prime}
$$

and the viscous solution $\hat{u}_{2}$ as $V_{4}$ where $V_{4}$ is given by, 


$$
v_{4}=\left(u_{2}, u_{2}, u_{2}, u_{2}, u_{4}\right)
$$

The method of : ution is to rirst obtain the viscous solution $V_{4}$. This can either ke evaluated for one integration step or across the range of integration and the valiues stored. The viscous solution is then normalised which we will denote by $\bar{X}_{1,}$, that is,

$$
V_{4}=\frac{V_{4}}{V_{4}}
$$

We now form the purified inviscid solution by setting,

$$
\bar{V}_{2}=\frac{V_{2}-\lambda V_{4}}{\left|V_{2}-\lambda V_{4}\right|}
$$

where the complex scalar $\lambda$ is given by,

$$
\lambda=V_{2} \cdot \bar{\nabla}_{4} *
$$

Where the star denotes the complex conjugate. The value of $\lambda$ may be eraluated at each step of the integration. The solutions $\bar{V}_{2}$ and $\bar{V}_{4}$ now represent the linearly independent inviscid and viscous solutions to the equation.

A numerical example of the above technique for a wholly real differential equation is given in Appendix $A_{0}$

\subsection{Numerical Calculation Procedure}

The numerical integration method chosen was the standard Runge- Kutta scheme with exror minimisation developed by fryfe (1965). It was found that the accuracy of the integration 
could be easily checked by altering the step size and comparing calculations, Tho numetical procedures were used in all; the fixst which checked e step length accuracy and recorded the required step length: the second used this information in an nnchecked numerical integration which saved computation time.

The integration is started from above the mixing layer where the exact solutions are known. To start the integration the coefricients $A_{2}$ and $A_{4}$, (equation $(5.7)$ ), are set equal to whity. If the starting value of $n^{*}$ is $n_{I}$ * then the starting conditions on $\hat{u}_{2}$ and $\hat{u}_{2_{4}}$ are

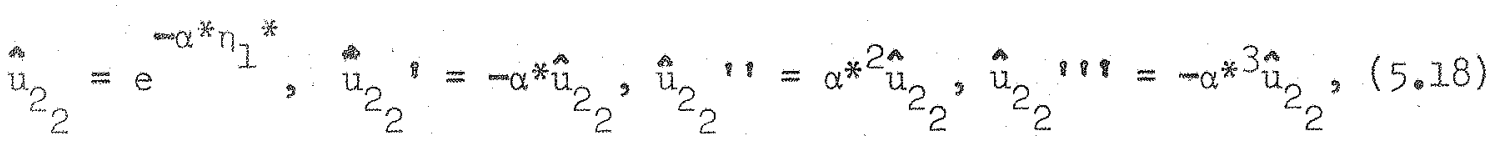

and

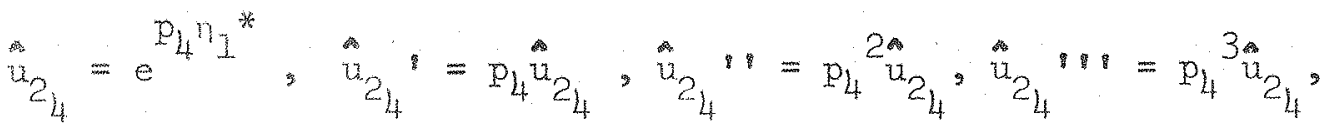

where primes denote afferentiation with respect to $n^{*}$.

For large values of Reynolds number the starting values or $u_{2}$ and its demivatives are very small, To prevent ploating point overflow occurring when the vector $V_{4}$ is normalised it is convenient to mutiply (5.19) by a suitably large constant throughout. The solution $W_{4}$ tends to grow very rapialy as the integration proceeds unless some scaling procedure is introm duced. The normalisation of $V_{4}$ at every integration step, which is required for the orthonormalisation procedure, prevents overfow of the viscous solution and no scaling factors are requirea. 
When an integration step is completed the solution $\mathrm{V}_{4}$ is nomalised and the ner thogonal vector $\bar{X}_{2}$ is calculated. the eigenfunctions are required the same transformations have to be perromed on the preceding values of $\bar{V}_{2}$ and $\bar{V}_{4}$. However if only the elgenvalues are required the time is saved if this backward transformation is not performed.

When the Iinearly independent solutions $\bar{V}_{2}$ and $\bar{V}_{4}$ have been calculated completely across the layer the boundary conditions below the layer must be applied to form the eigenvalue problem. Let the value of $n^{*}$ at the finish of integration below the layer be $\eta_{2} *$ Then at this point we have from equation $(5.9)$ $\left|\begin{array}{l}u_{2} \\ u_{2} \\ u_{2}\end{array}\right|=\left|\begin{array}{cccc}1 & 1 & 1 & 1 \\ \alpha^{*} & -\alpha^{*} & q_{3} & -q_{3} \\ u_{2}^{* 2} & \alpha^{* 2} & q_{3}^{2} & q_{3}^{2} \\ \alpha^{*} & -\alpha^{* 3} & q_{3}^{3} & -q_{3}^{3}\end{array}\right|(5.20)$

Equation (5.20) may be inverted to give four equations for $B_{n}$ in terms of $u_{2}$ and its derivatives. The equations for $B_{2}$ and $\mathrm{B}_{4}$ sre

$$
B_{2}=\frac{e^{\left(\alpha^{*} \eta_{2}^{*}\right)}}{\left.\left(2 \alpha^{*} q_{3}^{2}-\alpha^{*}\right)\right)}\left\{\hat{u}_{2}^{*}-\alpha^{*} \hat{u}_{2}^{*}-q_{3}^{2} \hat{u}_{2}^{*}+\alpha^{*} q_{3}^{2} \hat{u}_{2}\right\}
$$

and

$$
B_{4}=\frac{-e^{\left(q_{3} n_{2}^{*}\right)}}{\left(2 q_{3}\left(q_{3}^{2}-\alpha^{2}\right)\right)}\left\{u_{2}^{* n}-q_{3} u_{2} n-\alpha^{*} \hat{u}_{2}^{*}+q_{3} \alpha^{2} \hat{u}_{2}\right\}(5.22)
$$


The values of $\bar{V}_{2}$ are substituted into equations $(5.21)$ and (5.22) giving values of $B_{2}$ and $B_{4}$ which we will denote by $\left(R_{2}\right)$ and $\left(B_{4}\right)_{I}$. Similar substitution of $\bar{V}_{4}$ leads to values of $B_{2}$ and $B_{4}$ of $\left(B_{2}\right)$ II and $\left(B_{4}\right)$ II: Because the Orr Sommerfeld equation is linear solutions may be obtained by addition of the two solutions $\bar{V}_{2}$ and $\bar{V}_{4}$. The constants $B_{n}$ in the lower stream are thus given by

$$
B_{n}=A_{2}\left(B_{n}\right)+A_{4}\left(B_{n}\right) I I
$$

for arbitrary values of $A_{2}$ and $A_{4}$. We may set $A_{2}=1$ which is equivalent to dividing equation (5.7) throughout by $\mathrm{A}_{2}$. We require to find the values of $\mathrm{A}_{2}$ and $\mathrm{A}_{4}$ which will enable the boundary conditions to be satisfied below the mixing layer. Thus we first set

$$
A_{4}=\frac{-\left(B_{2}\right) I}{\left(B_{2}\right) I I}
$$

which determines the value of $\mathrm{A}_{4}$ for which $\mathrm{B}_{2}=0$. The constant $\mathrm{B}_{4}$ then becomes

$$
B_{4}=\left(B_{4}\right)_{I}-\frac{\left(B_{2}\right)}{\left(B_{2}\right)}\left(B_{4}\right)_{I I}
$$

For the boundary conditions to be met $B_{4}$ must vanish. The value of $B_{4}$ is set by the choice of parameters $\alpha, R$ and $c$ or $\omega, R$ and $c$, and these values must be altered in order to set $B_{4}=0$. This minimisation constitutes the numerical eigenvalue problem. In the case of temporal amplification it is convenient to keep $\alpha$ and $R$ constant and alter the value of $c$. For the spatial 
case we keep $w$ and $R$ constant and again alter the value of $c$. We may rewrite equation $(5.25)$ as

$$
B_{4}\left(R_{3}, \alpha, c\right)=\left(B_{4}\right) I-\frac{\left(B_{2}\right)_{I}}{\left(B_{2}\right)} \cdot\left(B_{4}\right)_{I I}
$$

The search for the zero of $B_{4}$ can be found by inear interpolation. As we are altening the value of $c$ only for a specitic set of values of $\alpha$ and $R$, or $w$ and $R$, we find the change in $B_{4}$ made by a small change in of $\delta c$. Then for any other small change in of $\Delta c$ we may write the value of $\mathrm{B}_{4}$ as,

$$
B_{4}(c+\Delta c)=B_{4}(c)+\frac{B_{4}(c+\delta c)-B_{4}(c)}{\delta c} \cdot \Delta c
$$

In order to make $B_{4}(c+\Delta c)$ zero we must therefore choose $\Delta c$ such that

$$
\Delta c=-\lambda\left\{\frac{B_{4}(c+\delta c)}{B_{4}(c)}-1\right\} \delta c_{9}
$$

where $\lambda$ is a factor that enables the computer to take large or small steps towards the minimum depending on the variation of $B_{4}$ with $c_{0}$. The value of $c$ is then replaced by $c+\Delta_{c}$ and $\delta_{c}$ by Wo where $\mu$ acts in the same way as $\lambda$ to reduce to step size as the minimum is reached.

When the computations were performed it was found to be very difficult to make $B_{4}$ exactly zero. Instead the program compares successive values of $c$ and when two such values are within a specified Imit then the interpolation is stopped. It was also found that for high Reynolds number the program would only converge if the initial gues at the eigenvalue is 
very close to the actual eigenvalue. This problem was also encountered by Sharma (1968) who developed the following scheme of inverse Langrangian interpolation which proved to give higher accuracy and faster convergence of the eigenvalue.

A rumber of values of $B_{4}$ for various values of $c$ are calculated and these values are used to generate a power series of the form,

$$
c_{n}=\sum_{n} a_{n} B_{4}^{n}
$$

An approximating series for $c_{n}$ is formed using Lagrange's interm polation polynomial and is of the form,

$$
c_{n+1}=\sum_{j=0}^{n} L_{j}{ }^{(n)}\left(B_{4}\right) \cdot c_{j}
$$

where

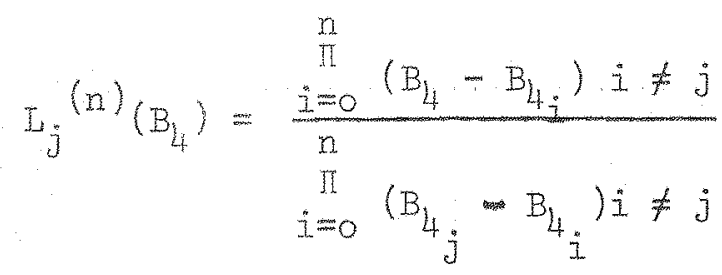

$I_{j}(n)$ is the $n^{\text {th }}$ Lagrangian coefficient formed for the $(n+1)$

$\operatorname{pairs}\left(c_{0}, B_{4}\right),\left(c_{1}, B_{4}\right), 0000000,\left(c_{n}, B_{4}\right)$.

We are seeking the value of $c$ for which $B_{4}$ is zero. Thus we set $B_{4}=0$ in $(5.30)$ and substitute into (5.29) giving,

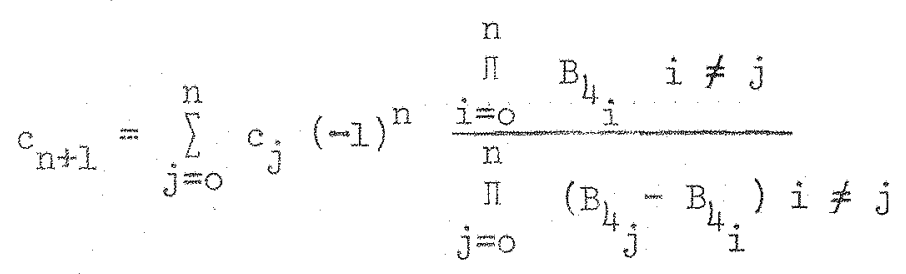

Equation 5.31) provides the next value of $c$ to be used in the solution of equation $(5.1)$. 
In the computations three values of $c$ are used to start giving thy ee correponding values of $\mathrm{B}_{4}$ and equetion (5. . . . used to determine the new value of $c_{\text {a }}$ The corresponding value of $B_{4}$ is calculated and the four sets of values are then used to determine another value for $c$. The convergence in our celculations appears to have been more rapid than that of Sharma. An exomple of the convergence of $B_{4}$ to a minimum is shown in Figure 10 ror a typical set of values of $R$ and $\alpha$ The surface $\left|B_{4}\right|$ as a function of complex variable c exhibits a sharp minimum at the eigenvalue but outside the minimum the surface gradually slopes away. If points are chosen outside the Irmediate area of the minimum then the interpolated new value of $c$ will not be closer to the eigenvalue and the procedure will not converge.

In the next section we will review previous work on the stability of free shear layers.

\subsection{Review of Studies of the Stability of Free Shear Layers}

The investigation of the stability of free shear layers was intitated by Lord Rayleigh (1880). He considered the Inviscid shear layer with a mean velocity profile given by,

$$
U=\left\{\begin{array}{cc}
1 & y>1, \\
y & -1<y<1, \\
-1 & y<1
\end{array}\right.
$$

He assumed that the value of $\mathrm{U}^{\mathrm{B}}$ was finite within the layer $y=1 \pm c$ and that ${ }_{2}$ was constant. Integrating the inviscid 
stability equation showed that the value of $(U-c) d_{2}^{\prime}-U^{\prime} \hat{u}_{2}$ at $y=1-\varepsilon$ must equal its value at $1+\varepsilon_{0}$. This lead to an expression for $c$ of the form,

$$
c^{2}=\left(1-\frac{1}{2 \alpha}\right)^{2}-\left(\frac{1}{4 \alpha^{2}}\right) e^{-4 \alpha}
$$

These values of c agree qualitatively with those obtained for continuous mean velocity profiles. The eigenvalues and amplifications for the profile (5.32) are sketched below。

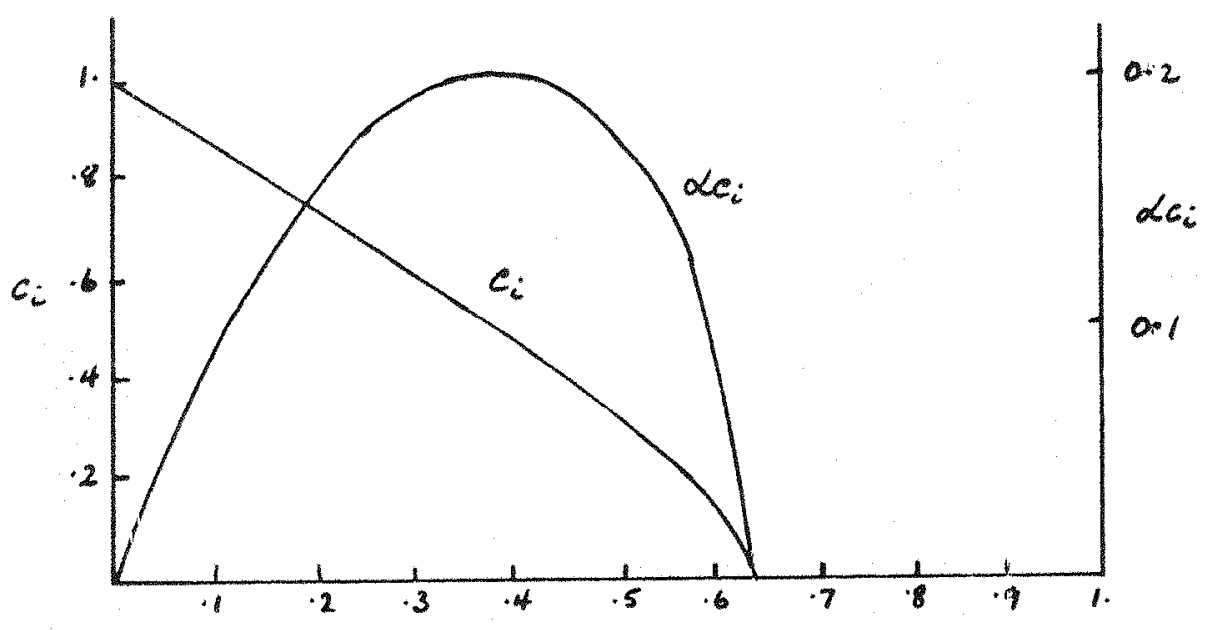

Eigenvalues and amplifications for discontinuous profile, equation (5.32)

Following Rayleigh's work the problem was not tackled again until 1950, when Lessen (1950) considered the free boundary layer between parallel streams. He solved the complete OrrSommerfeld equation using an expansion method similar to the one used to obtain the mean velocity profile. His calculations, and those of subsequent workers, showed that the unstable properties of the shear layer were very nearly independent of Reynoldis number for large values of Reynolds number。 Esch (1957) 
considered the stability of a free shear layer with a piecewise mean velocity profile, He showed that the neutral curve approached the origin with $\alpha=R \times$ const. This indicates that there exists no critical Reynolas number for the free shear layers. However for low values of Reynolds number the assumption of parallel mean flow is not valia as the variation in the mean velocity profile in the $x$-airection may be important. We will discuss this problem in more detail a little later. The inviscid shear layer has also been studied by Michalke (1964) who considered the disturbance vorticity distribution. He showed that for neutral disturbances a single concentration of vorticity existed within a single wave length forming a stable cell-Iike structure. However, for the case of maximum temporal amplifit cation two distinct concentrations existed within one wavelength. If these vorticity distributions are considered in terms of eddies then the instability may be seen as the rolling over of one eddy on another. The preceding investigations have all considered temporally growing disturbances as were assumed in the classical stability theory. In order to compare the results for temporal growth rates obtained theoretically with experimental results, a number of researchers, Schade and Michalke (1962) and Sato (1959) have used the disturbance phase speed to obtain spatial growth rates. The agreement was fair comparing wavenumbers and growth rates, other factors such as the eigenfunction for temporal amplification, was contrary to that predicted from experiment. As we have seen in Chapter 4, Gaster concluded that 
the phase velocity would only be used when the wave system is non-dispersive, which is nearly the case in boundary layers. Direct numerical calculations of the spatial growth rates involving complex wavenumber and real frequency have been made by Michalke (1965) for the inviscid shear layer, Betchov and Criminale (1966) for the inviscid jet and wake, Lessen and Ko (1966) for the viscous incompressible half jet flow and Ko and Lessen (1969) for the viscous full jet, The calculations by Michalke compared very well with the experimental results of Freymuth (1966) and Browand (1966)。 The growth rates and wavenumbers corresponded to the transformed values using temporal amplification. However, the essential features of the instability such as the distribution of the $u_{1}$-fluctuation, which exhibits a phase reversal for one stream having zero velocity, were also predicted. As we have noted, no critical Reynolds number is predicted using a parallel mean flow assumption. We will now discuss the modification in the growth rates caused by a non-parallel mean flow.

\subsection{The Stability of Non-Parallel Flows}

The non-paraliel mean flow problem was treated analytically by Lanchon and Eckhaus (1964). Theyconcluded that for free shear flows only the inviscid limit obtained from the quasiparallel flow assumption was correct and it would be essential to take account of flow divergence when viscous terms are included. They also noted that the asymptotic theory of weakly divergent flows is analytically little more complicated than the classical theory. 
In our direct numerical integration cf the stability equation we need to take account of the effect of flow divergence. Lessen and Ko (1966) and Lessen, Earpavat and Zien (1969) proposed a relatively simple method of correcting the stability curves in non-parallel flows. Though we shall note that some of their results are anomolous, it is felt that their basic argument is justified.

Lessen et. al, argue that in lirear stability theory it is the interaction energy between the disturbance and the main flow that plays a role in the stability of the flow. Let us denote this interaction energy by $\mathrm{E}(\mathrm{x}, \mathrm{y}, \mathrm{t})$ which will depend on the products of the disturbances. If we denote two such disturbance terms as $p(x, y, t)$ and $q(x, y, t)$ then their Fourier transforms are, $\hat{p}(y ; k, \omega)=\frac{1}{(2 \pi)^{2}} \int_{-\infty}^{\infty} \int_{-\infty} \exp \{i(k x-u t)\} p(y ; x, 1) d x d t$ and $\dot{q}(y ; k, w)=\frac{1}{(2 \pi)^{2}} \int_{-\infty}^{\infty} \int_{-\infty} \exp \{-i(k x-\omega t)\} q(y ; x, t) d x d t$, where $k$ is the wavenumber in the $x$-direction and $\omega$ is the angular frequency. The Fourier transform of the energy term will be of the form of the product of the two disturbances,

$$
\begin{gathered}
\stackrel{q}{q}(y ; k, i) \cdot \hat{p}(y ; k, w) \\
=\frac{1}{(2 \pi)^{4}} \int_{-\infty}^{\infty} \int_{-\infty} \exp \left\{-i(k x-\omega t) d x d t \int_{-\infty}^{\infty} p\left(x^{\prime}, y, t^{\prime}\right)\right. \\
q\left(\left(x-x^{\prime}\right), y\left(t-t^{\prime}\right)\right) d x^{\prime} d t^{\prime} \\
-104-
\end{gathered}
$$


Thus we may write the transform of the interaction energy as, $\mathbb{E}(y ; k, \omega)=\frac{1}{(2 \pi)^{2}} \int_{-(x)}^{\infty} \int_{-\infty} \exp \{-i(k x-\omega t)\} \mathbb{E}(y ; x, t) d x d t$

Let the energy density per unit width be given by $\varepsilon(y ; x, t)$ with its traxsform $\hat{\varepsilon}(\mathrm{y} ; \mathrm{k}, \omega)$ 。

Then we may write,

$$
\frac{1}{\varepsilon} \frac{d c}{d x}=\frac{I}{E} \frac{d E}{d x}-\frac{1}{V} \frac{d V}{d x}
$$

where $V$ is the volume ccupied by the disturbances: Let us assume that as the change in the volume $V$ depends on the growth of the layer then we may write,

$$
\frac{1}{V} \frac{d V}{d x}=\frac{I}{l} \frac{d \ell}{d x}
$$

where 2 is the width of the mixing layer for laminar flow such that,

$$
\ell=\left(\frac{v_{x}}{J_{d}}\right)^{\frac{1}{2}}
$$

From $(5.37)$ and $(5.38)$ we have,

$$
\frac{I}{l} \frac{d l}{d x}=\frac{I}{2 x} \quad \text {. }
$$

Making use of equation (5.35) we see that

$$
\frac{1}{\mathrm{E}} \frac{\mathrm{dE}}{\mathrm{dx}}=-\mathrm{k}_{i}
$$

where $k_{i}$ is the imaginary part of the wavenumber $k_{\text {. }}$ 
Substituting equations $(5.39)$ and $(5.40)$ into equation $(5.36)$ we obtain,

$$
\frac{I}{\varepsilon} \frac{d \hat{\varepsilon}}{d x}=-k_{i}-\frac{1}{2 x}
$$

Non-dimensionalising this equation with respect to the reference length $\ell$ and a reference energy density $\varepsilon_{0}$ we obtain,

$$
\frac{I}{\varepsilon^{*}} \frac{d \hat{\varepsilon}^{*}}{d x^{*}}=-\alpha_{i}-\frac{I}{2 R_{l}}
$$

where

$$
\begin{gathered}
\hat{\varepsilon}^{*}=\hat{\varepsilon} / \varepsilon_{0}, \quad \alpha_{i}=k_{i} \ell \text { and } \\
R_{\ell}=\frac{U_{d}^{\ell}}{v} .
\end{gathered}
$$

From equation (5.42) we see that the growth of the region has led to the inclusion of a term, $\left(2 R_{\ell}\right)^{-1}$, which reduces the amplification factor found from parallel flow assunptions. Thus for spatial amplification we may write,

$$
\left(a_{i}\right)_{\text {non-parallel }}=\left(\alpha_{i}\right)_{\text {parallel }}+\frac{1}{2 R_{\rho}}
$$

This result is the same as that obtained by Lessen and Ko (1966)。 However, it is interesting to note that if one assumes a wavelike form for the disturbances a priori, then the interaction energy will have an exponent of twice that of the disturbance. However, naking use of the Fourier transform, as we have seen above, eliminates this problem.

Let us now consider the effect of a divergent flow when one considers temporal amplification. Using a similar technique to that above we may write 


$$
\frac{1}{\varepsilon^{*}} \frac{d^{*} \varepsilon^{*}}{d t^{*}}=\alpha c_{i}-\frac{1}{2 R_{l}}: \frac{d x^{*}}{d t^{*}}
$$

In their discussion of the probiem, Iressen et. al. (1969) set $\frac{d x^{*}}{d t *}$ equal to unity which implies that the disturbances are travelling at the mean reference velocity.

Let us set $\frac{d x^{*}}{d t^{*}}=c^{*}$, a non-dimensionalised velocity which represents the translation speed of the disturbances. We may then write,

$$
\left(\alpha c_{i}\right)_{\text {non-parallel }}=\left(\alpha c_{i}\right)_{\text {paraliel }}-\frac{c^{*}}{2 R_{l}}
$$

The neutral stability curves for either spatial or temporal amplification must be identical in the non-divergent case as well as the parallel case. Making use of our discussion of spatial amplification in Chapter 4, we may usefully multiply equation (5.43) by $-\frac{\partial \omega_{r}}{\partial \alpha_{r}}$ to obtain,

$$
\left(\alpha_{i} \frac{\partial \omega_{r}}{\partial \alpha_{r}}\right)_{\text {non-parallel }}=\left(\alpha_{i} \frac{\partial \omega_{r}}{\partial \alpha_{r}}\right)_{\text {parallel }}+\frac{1}{2 R_{l}} \cdot \frac{\partial \omega_{r}}{\partial \alpha_{r}} \quad(5.46)
$$

Now from equation (4.72) we have,

$$
\frac{\omega_{i_{T}}}{\alpha_{i_{S}}}=-\frac{\partial \omega_{r}}{\partial \alpha_{r}}
$$

where the subscripts $T$ and $s$ refer to temporal and spatial amplification respectively. From equations (5.45) and $(5.46)$ with (4.72) we may see that

$$
c^{*}=\frac{\partial \omega_{r}}{\partial \alpha_{r}}=c_{g} \text {, the group velocity }
$$


In the case of a non-dispersive wave system the group velocity will equal the wave speed given by

$$
c_{r}=\frac{\omega_{r}}{\alpha_{r}}
$$

Thus we may see from the preceding discussion that we may only calculate the correction in amplification factor for the temporal case when the system is non-dispersive and then we have,

$$
\left(\alpha c_{i}\right)_{n o n-p a r a l l e I}=\left(\alpha c_{i}\right)_{\text {parallel }}-\frac{c_{r}}{2 R_{\ell}}
$$

\subsection{Results and Discussion}

In this section we will present the numerical results for the stability of a mixing layer between two streams. Calculations have been performed for the two cases of temporal and spatial amplification. "The results for the temporal case will be presented first and comparison will be made, where possible, with the results of other researchers.

\subsubsection{Eigenvalues and Eigenfunctions for the Temporal Case}

Calculation of the eigenvalues for the temporal case were made for the laminar velocity profile calculated in Chapter 3 for the mixing of two streams with finite velocity. The velocity ratio of the two streams was 0.61 . The eigenvalues for various wavenumbers and Reynolds numbers are tabulated below. The results of Esch (1.957) indicated that for values of $\alpha R>100$ the results of inviscid theory were found to be a good approximation to the viscous results. Calculations were thus made at a value of $R_{\theta}=500$ so that comparison with the results of inviscid theory 
and calculation could be made. The value of 500 was chosen so as to clearly meet the requirement of Esch and also so that computations could be faster than if a higher value of Reynolds number had been chosen. The eigenvalues were evaluated by integrating between values of $y / \theta$ of +2 and -2 , with a step size of 005 . In all cases the computation was completed when successive values of the interpolated eigenvalue differed by less than $10^{-7}$.

TabIe III. Numerical Results of the CaIcuIations. Temporal Case

\begin{tabular}{rcccc}
$R_{\theta}$ & $\alpha$ & $c_{r}$ & $c_{i}$ & $\alpha c_{i}$ \\
5.0 & .2 & 4.068748 & .349364 & .069873 \\
5.0 & .4 & 4.070216 & .289218 & .075687 \\
5.0 & .55 & 4.071635 & .073067 & .040187 \\
\hline 10.0 & .2 & 4.068189 & .474343 & .094869 \\
10.0 & .4 & 4.070206 & .350662 & .140265 \\
10.0 & .6 & 4.072800 & .223019 & .133811 \\
10.0 & .8 & 4.075939 & .098330 & .078664 \\
\hline 12.5 & .2 & 4.068031 & .509870 & .101974 \\
12.5 & .4 & 4.070301 & .392735 & .157094 \\
12.5 & .6 & 4.073127 & .0269509 & .161705 \\
12.5 & .8 & 4.076464 & .148938 & .119151 \\
\hline 15.0 & .2 & 4.067927 & .537436 & .107487 \\
15.0 & .4 & 4.070428 & .424085 & .169634 \\
15.0 & .6 & 4.073447 & .303373 & .182024 \\
15.0 & .8 & 4.076943 & .185258 & .148207 \\
\hline
\end{tabular}


$R_{\theta} \quad \alpha$

(1)

$c_{r}$

$c_{i}$

$\alpha c_{i}$

\begin{tabular}{ccccc}
500.0 & 0.1 & 4.068741 & .876765 & .087676 \\
500.0 & 0.2 & 4.072069 & .808464 & .162693 \\
500.0 & 0.4 & 4.076671 & .667809 & .267124 \\
500.0 & 0.6 & 4.081002 & .538959 & .323375 \\
500.0 & 0.8 & 4.085574 & .421458 & .337166 \\
500.0 & 1.0 & 4.090603 & .312914 & .312914 \\
500.0 & 1.2 & 4.096279 & .211166 & .253399 \\
500.0 & 1.3 & 4.099424 & .162251 & .210927 \\
500.0 & 1.4 & 4.102813 & .114379 & .160131 \\
500.0 & 1.5 & 4.106474 & .067347 & .101020 \\
500.0 & 1.6 & 4.110444 & .020994 & .033591 \\
500.0 & 1.7 & 4.114761 & -.024834 & -.042218 \\
\hline
\end{tabular}

The temporal amplification factors $\alpha c_{i}$, are shown graphically in Figure 11 as a function of the wavenumber. The cal.culations of Betchov and Szewczyk (1963) for a hyperbolic tangent representation of the mean profile are shown as a dotted line. The results of Rayleigh (1880) for the mean velocity profile given by equation (5.32) are also plotted. These two calculations are for the inviscid shear layer. It can be seen that for low wavenumbers the amplification factors are nearly independent of the mean profile shape. This agrees with the work of Esch (1957) 
and Michalke (1969). Esch comments that at low wavenumbers the wavelength of the disturbance will be large compared with the width of the shear layer and the disturbance motion will spread deeply into the uniform streams outside the shear layer. Under these conditions the individual mean velocity profiles will all appear the same and the amplification will be inopendent of the profile shape. However, at high wavenumbers the individual mean velocity profile greatly affects the stability characteristics of the shear layer. It can be seen that at a Reynolds number of 15 the maximum amplification factor is 55\% of the maximum value at the high Reynolds number. It will be seen later that when. corrections are made for the flow divergence then the amplification factors at low Reynolds numbers are reduced.

Curves of constant amplification factor are plotted in Figure 12. It can be seen from the figure that there exists no critical Reynolds number, that is no Reynolds number below which no amplification occurs. In his analysis of the instability of the shear layer with mean velocity profile given by equation (5.32), Esch (1957) showed that for small wavenumbers a form of the secular determinant equation (4.11) led to a series in $\left(\alpha \mathrm{R}_{\theta}\right)^{\frac{1}{2}}$, for the neutral stability case. Equating the first non-vanishing term in the series to zero yielded the equation,

$$
2 i \delta\left[\delta+\left(\delta^{2}-i\right)^{\frac{1}{2}}\right]\left[\delta+\left(\delta^{2}+i\right)^{\frac{1}{2}}\right]+\left(\delta^{2}-i\right)^{\frac{1}{2}}-\left(\delta^{2}+i\right)^{\frac{1}{2}}=0,(5.50)
$$

where $\delta=\alpha /\left(\alpha \mathrm{R}_{\theta}\right)^{\frac{1}{2}}$. For positive real $\delta$ this equation has the single root, 


$$
\delta=380 \text { 。 }
$$

The equation, $\alpha=.380\left(\alpha \mathrm{R}_{\theta}\right)^{\frac{3}{2}}$,

is plotted in Figure 13 and compared with our calculated neutral stability curve replotted as a function of $\left(\alpha R_{\theta}\right)$. It can be seen that for values of $\alpha R_{\theta}<1$ the agreement is good. This also serves to demonstrate that for low wavenumbers the velocity profile shape does not affect the overall stability of the shear layer. It is interesting to note that our calculations give no indication of a peak in the neutral stability curve about $\left(\alpha \mathrm{R}_{\theta}\right)$ 15 as was calculated by Esch. The monotonicity of the curve is later demonstrated again for the case of spatial amplification. The wave speed $c_{r}=\omega_{r} / \alpha_{r}$ is plotted as a function of wavenumber in Figure 14. It can be seen that for the case of an assymmetric velocity profile the wave speed is a function of wave number. For symmetric profiles it may be shorn that the value of $c_{r}=\frac{1}{2}\{U(\infty)+U(-\infty)\}=U_{S}$. That is that er a symmetric profile $c_{x}=0$. In this case $U(-y)=-U(y)$ and if we replace $y$ by $-y$ in the orr-Sommerfeld equation we obtain,

$$
\begin{aligned}
& \hat{u}_{2}^{i v}(-y)-2 \alpha^{2} \hat{u}_{2}^{\prime \prime}(-y)+\alpha^{4} \hat{u}_{2}(-y) \\
& =-i \alpha R\left[(u+c)\left\{\hat{u}_{2}(-y)-\alpha^{2} \hat{u}_{2}(-y)\right\}-U \cdot \hat{u}_{2}(-y)\right]
\end{aligned}
$$

Then if we put $x(y)=\hat{u}_{2}^{*}(-y)$, (see Tatsumi and Gotoh (1959)), where the star denotes the complex conjugate, then takirg the complex conjugate of equation (5.52) leads to,

$$
x^{i v}-2 \alpha^{2} x^{\prime \prime}+\alpha^{4} x=\operatorname{i\alpha R}\left\{\left(U+c^{*}\right)\left(x^{\prime \prime}-\alpha^{2} x\right)-U^{\prime \prime} x^{\prime},\right.
$$


which equation in $x(y)$ is identical with the orr-bommerfeld equation for $\vec{u}_{2}(y)$ when $c=-c^{*}$. If for a given $\alpha$ and $R$ there exists a unique solution then the eigensolutions $x$ and $\phi$ must be equal and $c=-c^{*}$, which leads to

$$
c_{r}=0 \text {. }
$$

From Figure 14 we may see that for low wavenumbers the value of $c_{r}$ is approximately equal to the average velocity of the two strems, again showing the lack of influence of the profile shape at Iow wave numbers.

The eigensolutions $\hat{u}_{2}(y / \theta)$ and their first derivatives are plotted in Figures $15(a)$ and $(b)$ and 15(c) and (d) for several wavenumbers and a value of $R_{\theta}=500$. The functions have been normalised such that $\hat{u}_{z_{r}}=1$ and $\hat{u}_{z_{i}}=0$ when $(y / \theta)=0$. It is interesting to note that the distributions are not symetric about the $x$-axis in the temporal case. The derivative of the eigenfunction is related to the axial velocity disturbance through the transformed equation of continuity,

$$
\hat{u}_{1}=\frac{i \hat{u}_{2}}{\alpha}
$$

The amplitude distributions of axial and transverse velocity fluctuations are shown in Figures 16 and 77 respectively. The distributions have been normalised such that the value of $\left|\hat{u}_{I}\right|^{2}$ is unity for $y / \theta$ equal to zero. We can see that the axial velocity fluctuation distributions exhibit three distinct peaks. The magnitude of these peaks increases as the wavenumber increases. By comparing Figures 16 and 17 we can see that the relative magnitudes of the axial to the transverse fluctuations decrease as the wavenumber increases.

$$
-113-A \text {. }
$$


The relative phase and amplitude of the computed eigenfunctions and derivatives for two wavenumbers are shown in Figures 18 and 19. For a wavenumber of 1.0 the two outside peaks which occur on the $\left|\hat{u}_{1}\right|^{2}$ aistribution may be seen to be approximately $\pi$ radians out of phase with each other. For the same wavenumber the central peak in the distribution is $\pi / 2$ radians leading the peak at negative $y / \theta$ and $\pi / 2$ radians lagging the peak at positive $y / \theta$. As the wavenumber decreases so the phase difference between the three peaks increases. We will now consider the results of the calculations for spatial amplification. Subsequently we will evaluate the corrections to the stability curves for divergent flow, then, where possible, compare the results of computations in both the temporal and spatial cases with other theory and experiment.

\subsubsection{If genvalues and Eigenfunctions for the Spatial Case}

Calculations made for the spatial case were performed at similar Reynolds numbers to those of the temporal case. For the reasons given in the previous section, the maximum value of Reynolds number chosen was 500. The eigenvalues were evaluated by integrating between values of $y / \theta$ of +2 and -2 , with a step size of .005 . The same limits of convergence were used as for the temporal case. The eigenvalues for various frequencies and Reynolds numbers are tabulated below. 
Table IV Numerical Results of CaIculations - Spatial Case

\begin{tabular}{|c|c|c|c|c|c|}
\hline $\mathrm{R}_{\theta}$ & (w) & $c_{r}$ & $c_{i}$ & $\alpha_{r}$ & $\alpha_{i}$ \\
\hline 5.0 & .25 & 4.059658 & .468662 & .060772 & -.007016 \\
\hline 5.0 & .50 & 4.057460 & .414033 & .121960 & -.012445 \\
\hline 5.0 & .75 & 4.055135 & .362751 & .183482 & -.016413 \\
\hline 5.0 & 1.00 & 4.053769 & .312416 & .245227 & -.018899 \\
\hline 5.0 & 1.25 & 4.053587 & .262667 & .807079 & -.019898 \\
\hline 5.0 & 1.50 & 4.054612 & .213512 & .368926 & -.019427 \\
\hline 5.0 & 1.75 & 4.056799 & .165028 & .430662 & -.017519 \\
\hline 5.0 & 2.00 & 4.060076 & .117287 & .492191 & -.014218 \\
\hline 5.0 & 2.25 & 4.064363 & .070344 & .553426 & -.009578 \\
\hline 5.0 & 2.50 & 4.069582 & .024235 & .614292 & -.003658 \\
\hline 5.0 & 2.75 & 4.075657 & -.021022 & .674720 & .003480 \\
\hline 10.0 & .25 & 4.063003 & .542055 & .060454 & -.008065 \\
\hline 10.0 & .50 & 4.059945 & .516489 & .121193 & -.015418 \\
\hline 10.0 & .75 & 4.055824 & .484400 & .182319 & -.021775 \\
\hline 10.0 & 1.00 & 4.052309 & .448453 & .243787 & -.026979 \\
\hline 10.0 & 1.25 & 4.049801 & .410550 & .305520 & -.030957 \\
\hline 10.0 & 1.50 & 4.048382 & .371126 & .367431 & -.033683 \\
\hline 10.0 & 1.75 & 4.048026 & .331424 & .429431 & -.035159 \\
\hline 10.0 & 2.00 & 4.048665 & .291658 & .491440 & -.035402 \\
\hline 10.0 & 2.25 & 4.050216 & .252093 & .553382 & -.034444 \\
\hline 10.0 & 2.50 & 4.052601 & .212900 & .615190 & -.032318 \\
\hline 10.0 & 2.75 & 4.055743 & .174189 & .676802 & -.029068 \\
\hline 10.0 & 3.00 & 4.059576 & .136027 & .738165 & -.024734 \\
\hline
\end{tabular}




\begin{tabular}{|c|c|c|c|c|c|}
\hline$R_{\theta}$ & $\omega$ & $c_{r}$ & $c_{i}$ & $\alpha_{r}$ & $\alpha_{i}$ \\
\hline 10.0 & 3.25 & 4.064043 & .098454 & .799227 & -.019362 \\
\hline 10.0 & 3.50 & 4.069093 & .061492 & .859946 & -.012996 \\
\hline 10.0 & 3.75 & 4.074684 & .025147 & .920282 & -.005679 \\
\hline 10.0 & 4.00 & 4.080779 & -.010582 & .980198 & .002542 \\
\hline 12.50 & .75 & 4.056048 & .519310 & .181927 & -.023293 \\
\hline 12.50 & 1.50 & 4.047024 & .412710 & . 366828 & -.037409 \\
\hline 12.50 & 2.25 & 4.047373 & .297750 & .552924 & -.040677 \\
\hline 12.50 & 3.00 & 4.055179 & .185103 & .738257 & -.033699 \\
\hline 15.0 & .25 & 4.064982 & .584232 & .060256 & -.008660 \\
\hline 15.0 & .50 & 4.061245 & .571603 & .120724 & -.016991 \\
\hline 15.0 & .75 & 4.056151 & .546030 & .181607 & -.024470 \\
\hline 15.0 & 1.00 & 4.051677 & .515140 & .242885 & -.030881 \\
\hline 15.0 & 1.25 & 4.048266 & .480384 & .304487 & -.036132 \\
\hline 15.0 & 1.50 & 4.045994 & .443854 & .366328 & -.040187 \\
\hline 15.0 & 1.75 & 4.044815 & .406472 & .428327 & -.043044 \\
\hline 15.0 & 2.00 & 4.044645 & .368801 & .490404 & -.044716 \\
\hline 15.0 & 2.25 & 4.045386 & .331190 & .552486 & -.045231 \\
\hline 15.0 & 2.50 & 4.046950 & .293861 & .614509 & -.044621 \\
\hline 15.0 & 2.75 & 4.049252 & .256952 & .676414 & -.042923 \\
\hline 15.0 & 3.00 & 4.059920 & .149433 & .860920 & -.031688 \\
\hline 15.0 & 3.75 & 4.064553 & .114756 & .921876 & -.026028 \\
\hline 20.0 & .75 & 4.056111 & .587137 & .181111 & -.026217 \\
\hline 20.0 & 1.50 & 4.044434 & .488145 & .365555 & -.044121 \\
\hline 20.0 & 2.25 & 4.042706 & .377540 & .551746 & -.051526 \\
\hline
\end{tabular}




\begin{tabular}{|c|c|c|c|c|c|}
\hline$R_{\theta}$ & $\omega$ & $c_{r}$ & $c_{i}$ & $\alpha_{r}$ & $\alpha_{i}$ \\
\hline 20.0 & 3.00 & 4.048436 & .268907 & .0737772 & -.049004 \\
\hline 20.0 & 3.75 & 4.059543 & .165211 & .922222 & -037532 \\
\hline 20.0 & 5.00 & 4.086860 & .004075 & 1.223432 & -.001220 \\
\hline 500.0 & 0.10 & 4.068019 & .881803 & .023478 & -.005089 \\
\hline 500.0 & 0.15 & 4.067089 & .894179 & .035181 & -.007735 \\
\hline 500.0 & 0.25 & 4.062960 & .895915 & .058678 & -.012939 \\
\hline 500.0 & 0.50 & 4.053139 & .865303 & .117984 & -.025188 \\
\hline 500.0 & 0.75 & 4.045608 & .823418 & .178012 & -.036231 \\
\hline 500.0 & 1.50 & 4.033164 & .692261 & .361273 & -.062010 \\
\hline 500.0 & 2.25 & 4.031221 & .569186 & .547234 & -.077266 \\
\hline 500.0 & 3.00 & 4.035964 & .456336 & .733934 & -.082984 \\
\hline 500.0 & 3.75 & 4.045310 & .352752 & .920004 & -.080225 \\
\hline 500.0 & 5.00 & 4.067968 & .196176 & 1.226263 & -.059136 \\
\hline 500.0 & 5.25 & 4.073400 & .166850 & 1.286691 & $-.05270_{4}$ \\
\hline 500.0 & 5.50 & 4.079111 & .138093 & 1.346790 & $-.045594_{4}$ \\
\hline 500.0 & 5.75 & 4.085103 & .109884 & 1.406536 & -.037834 \\
\hline 500.0 & 6.00 & 4.091371 & .082173 & 1.465910 & -.029442 \\
\hline 500.0 & 6.25 & 4.097918 & .054944 & 1.524891 & -.020445 \\
\hline 500.0 & 6.50 & 4.104746 & .028175 & 1.583458 & -.010869 \\
\hline 500.0 & 6.75 & 4.111857 & .001846 & $1.64159 \%$ & -.000737 \\
\hline
\end{tabular}

The spatial amplification factor, $-\alpha_{i}$, is plotted against frequency for several Reynolds numbers in Figure 20. Also shown in the figure is the amplification curve for the temporal case simply transformed for comparison with the spatial case. 
The spatial amplification factor, $-\alpha_{i}$, and the temporal factor $-\alpha c_{i} / c_{r}$ may be seen to coincide very closely. This, as we will discuss later, is unlike the results of Michalke (1965) whose calculations produced only broadly similar curves for the case of an anti-symmetric mean velocity profile and one free stream at rest. Curves of constant amplification factor are shown in Figure $2 \mathrm{z}$ as a function of wavenumber and Reynolds number. As may be seen from an inspection of the eigenvalue problem in both the temporal and spatial cases, the neutral stability curve is the same in both. In the neutral case the wavenumber, frequency and eigenvalue are all real. Also shown in the figure is the curve

$$
\alpha=\frac{1}{4 \sqrt{3}} R_{\theta},
$$

which is the neutral stability curve at low wavenumbers derived in this torm by Tatsumi and Gotoh (1960) being equivalent to equation (5.51).

The wave speed $\omega_{r} / \alpha_{r}$ is plotted in Figure 22 as a function of frequency for several Reynolas numbers. Also show for the high Reyrolds number case is the corresponding wave speed curve for the temporal amplification case. The noticeable aifference between the two cases is the rise in the magnitude of the wave speed in the spatial case at low frequencies. However, it is to be noticed that there exists a maximum value of wave speed of 4.2633. In fact the wave speed in the spatial case varies between 50 and 60 percent. of velocity difference above the velocity of the lower velocity free stream. In his calculations 
for the separated shear layer, i.e. $U_{2}=0$, Michalke (1965), using inviscid theory, calculated that at zero frequency the wave speed equalled the velocity of the free stream $U_{I}$. The eigenfunctions and their derivatives are shown in Figures $23(a)$ and $(b)$ and $23(c)$ and (a) respectively. As in the temporal case the eigenfunctions have been nomalised such that $\hat{u}_{2_{x}}=I$ and $\hat{u}_{2_{i}}=0$ when $y / \theta=0$. It is interesting to note the close correspondence between the eigenfunctions in the temporal and spatial cases at approximately corresponding wavenumber and frequencies. This may also be seen in the amplitude distributions of axial and transverse velocity fluctuations shown in Figures 24 and 25 respectively. The relative phase of the $\hat{u}_{1}$ fluctuation is shown in Figure 26 for several frequencies. It can be seen that the overall phase difference across the layer is less for the higher frequencies than the Iower. Also the major phase change, which occurs towards the centre of the mixing layer, takes place over a narrower region for the higher frequency fluctuations than for the lower frequency disturbances. Also shown in the figure is the calculated phase distribution of Freymuth (1966) and Michalke (1965) for the case of $U_{2}=0$ and inviscid flow. In their calculations of the spatial case they found a sharp phase reversal corresponding to the sharp minimum in the $\hat{u}_{1}$ magnitude distribution. This they also observed experimentally and the reversal occurred at all frequencies. In our calculations of the spatial case we have found no phase reversal. 
It may be argued that the case of a mixing layer with one stream at rest is the special case in the general problem of the mixing of two streams with finite velocity. Only when both streams have firite velocity can the assumption that the disturbances are much smaller than the mean quantities be applied everywhere in the flow. Thus one must question the validity of linearised theory in the special case. However, the calculations and observations of Freymuth do coincide. We may argue that by posing the eigenvalue problem as in equation $(5.6)$ the results for spatial and temporal calculations will coincide as $U_{2}$ tends to equal $U_{1}$. We may see this if we consider the imaginary part of the frequency which, for spatial amplification, is to be zero. The imaginary part of the frequency is

$$
w_{i}=\alpha_{i} c_{r}+\alpha_{r} c_{i}
$$

Making use of equation (5.6a) we may write this as,

$$
\omega_{i}=\alpha_{i} \frac{U_{S}}{U_{d}}+\alpha_{i} c_{r}^{*}+\alpha_{r} c_{i}^{*}
$$

For spatial amplification $\omega_{i}=0, \alpha_{i}$ is negative and $c_{i}{ }^{*}$ is positive. Thus for positive real part of wavenumber we have,

$$
\alpha_{i}=-\left(\alpha_{r} c_{i}^{*}+\alpha_{i} c_{r}^{*}\right) \cdot \frac{U_{d}}{U_{S}}
$$

Now for antisymmetric or nearly antisymmetric mean velocity profiles the value of $c_{r}$ * is either identically zero or close to zero respectively. Thus we obtain

$$
\alpha_{i}=-\alpha_{r} c_{i} * \frac{U_{d}}{U_{s}}
$$


Assuming, as the calculations indicate, the values of $\alpha_{r}$ and $c_{i}$ * remain finite as $U_{2}$ tends to $U_{1}$ then towards the linit as $U_{2}$ tends to $U_{1}, \alpha_{i}$ tends to zero. That is the spatial and temporal cases tend to coincide. Thus one would expect that the results for the spatial case will change from those for the spatial case of $\mathrm{U}_{2}=0$, calculated by Michalke, to those of the temporal case as $U_{2}$ tends to $U_{1}$. As of yet there exists no experimental verification of this as all experiments on the transition of a free shear layer have dealt with the case of $U_{2}=0$.

Experimental results have indicated that the mode which dominates the flow in the region of validity of the linearised theory corresponds to the mode at the frequency at which maximum amplification occurs. From the results of Michalke (1965) and Sato $(1956,1959)$ for the case of one stream at rest, it may be inferred that the spatial amplification case is to be considered. The distributions of the magnitude of the axial and transverse velocity fluctuations are shown in Figures 27 and 28 respectively for cases of both temporal and spatial amplification. The results have been normalised such that $\left|\hat{u}_{1}\right|^{2}$ is unity when $y / \theta$ is zero. It can be seen that the differences between the two cases of amplification are slight. The maximum amplitude of the axial velocity fluctuation is larger in the spatial case, though the maximum amplitude of the transverse velocity fluctuation is greater in the temporal case. We may also note that the minimum in the $\left|\hat{\mu}_{1}\right|^{2}$ distribution occurring towards the lower velocity stream has a lower value in the spatial than in the temporal case. This may suggest that, as 
we have argued above, this minimum will continue to decrease as the value of $U_{2}$ decreases until, when $U_{2}=0$, a phase reversal will be evident. The two outside peaks in the $\left|\hat{u}_{1}\right|^{2}$ aistribution occur at the very edges of the mixing layer as may be seen from the mean velocity profile plotted in Figure $\mathrm{J}$. The peaks occur at 8.5 percent. and 99.5 percent. of the velocity difference. This corresponds to the results of Freymuth who also noted that the outside peaks move inward as the frequency increased. The maximum value of the $\left|\hat{u}_{1}\right|^{2}$ fluctuation is approximately twice the maximum value of the $\left|\hat{u}_{2}\right|^{2}$ fluctuation in both cases.

From equations $(5.7)$ and $(5.8)$ we may see that for high Reynolds numbers the eigenfunction will behave as $u_{2} \alpha e^{ \pm \alpha y}$ as the mean velocity approaches that of either stream. The sign of the exponent is chosen such that the function is bounded. From equation (5.55) we may then write

$$
\begin{aligned}
\hat{u}_{1} & =i \hat{u}_{2} \\
\text { i.e. }\left|\hat{u}_{1}\right|^{2} & =\left|\hat{u}_{2}\right|^{2} .
\end{aligned}
$$

This result holds in our calculations for $\mathrm{y} / \theta>1.3$ ano $\mathrm{y} / \theta<$ I.3. The values of $f^{\prime}(y / \theta)$ at these values of $y / \theta$ are +1.0 and -0.9 respectively.

\subsubsection{Corrections for Non-Parallel Flow}

The eigenvalues obtained for the parallel flow case are modified for the non-parallel flow case according to equation (5.43) and the modified curves of constant amplification factor for the spatial case are plotted in Figure 29. As may be seen 
from equation (5.43) the correction is a function of the Reynolas number and decreases as the Reynolds number increases. It may be seen that the modification for non-parallel. flow has led to a non-zero value for the critical Reynolds number. This value is 12.3 and agrees with the value of $R_{C R I T}$ of 12 calculated by Lessen and Ko (1966) for the half-jet flow. We have seen from the comparison of the spatial amplification factors and the temporal amplification factors divided by the wave speed that the argument leading to equation (5.49) may be tentatively aprlied in these calculations. Making use of equation (5.49) the curves of constant amplification, modified for nor-parallel flow in the tempral case, are plotted in Figure 29. As in the spatial case a non-zero value of critical Reynolds number of 12.3 is obtainea. This is to be expected due to the coincidence of the neutrol curves in the parajlel flow case and the correspondance of the spatial and temporal amplification factors.

\subsection{Conclusions}

In this chapter we have examined the stability of a mixing layer between two parallel streams. We have seen that for the case of both free streams having finite velocity the corresponding eigenvalues and eigenfunctions for both temporal and spatial amplification are similar. When the two streams tend to have equal velocity then the temporal and spatial cases tend to coincide. We have noted that viewed in this way the problem of $\mathrm{U}_{2}$ being zero is a special case of the general mixing problem and its results do not apply when $U_{2}$ is non-zero. Making use 
of a non-parallel flow correction based on the energy of the interaction between the disturbances and the flow a critical Reynolds number of 12.3 has been calculated.

In the next chapter we will consider the fully turbulent mixing problem. It will be seen that equations of the type used in this chapter to determine the stability of a laminar flow may also occur in the turbulent case. 


\section{CHAPTTER 6}

\section{A Model of the Turbulent Mixing Layer Between Two Streams}

In this chapter we will"propose a model for the structure of a turbulent mixing layer between two streams. Although the mathematical analysis is similar to that in our previous discussion of the stability of the mixing layer, this analysis will be duplicated in part in order that the two problems may be considered entirely separately.

Firstly we will state the physical structure of the model to be used. In our subsequent mathematical derivation the points will be reiterated where they enter the mathematical model. It is considered that the large scale eddies in the shear flow will dominate the flow structure. These large scale motions are related to the most amplifying disturbances in the flow. The action between the large eddies and the mean flow causes further growth of this scale of motion. The interaction between the large scale motion is then considered to produce smaller scales which contribute to a background turbulence. This background motion may be connected with a turbulent eddy viscosity, which may or may not take the same value as the eddy viscosity used in mean flow analysis. The effect of the eddy viscosity is to reduce the effective Reynolds number of the flow which has a stabilising or amplitude limiting effect on the large scale motions. Thus we see the model is closed and demonstrates the dual. rôle of non-linearity in turbulence. Primarily the action between mean flow and large scale motions has a forcing 
function effect on the turbulence. Secondly the interaction between edaies leads to a background turbulence which reduces the effective Reynolds number and limits the amplitude of the disturbances.

Let us now derive the governing differential equations for the problem.

\section{I Governing Differential Equations}

Let us consider the mixing of two parallel streams of velocity $U_{1}$ and $U_{2}$ above and below the mixing layer. Let the $x_{1}$-co-ordinate lie in the initial direction of flow of the two streams and the $x_{2}$-co-ordinate be across the mixing layer. We will assume that the mixing begins at the origin and that the mean flow is two-dimensional. As the analysis associated with the derivation of our final equations is very complicated it is thought to be useful to introduce two assumptions at this stage.

We will firstly assume that the mean flow is parallel. In our discussion of the stability of the mixing layer we have seen that the growth and form of fluctuations in a non-parallel flow are very similar to that in a parallel flow except at low Reynolds numbers. In the case of a turbulent mixing layer the Reynolds number of the flow is high enough such that the assumption of parallel mean flow will not affect our predictions of the turbulent structure. We will also assume that the disturbances in the flow are two-dimensional. Though it is recognised that the fluctuations in the flow are three-dimensional it will be assumed that the dominating motions are in two dimensions. 
We have seen that in the problem of stability in a mixing layer the dominating disturbances are two-dimensional, that is the more oblique the wave is the less fast will it grow. We will see in our discussion of the turbulent flow case that similar differential equations govern the fluctuations in a turbulent flow and in a laminar flow on the point of transition. Michalke (1969) has shown numerically that this dominance of the two dimensional mode applies whether fluctuations are considered as having growth or decay with time or space. This assumption of two-dimensionality could be introduced subsequent to our derivation of the equations rather than here, but the increased complexity of the analysis is not considered to justify this exactness.

With the assumption of two dimensional disturbances, i。e. $u_{3}=0$ and $\frac{\partial}{\partial x_{3}}=0$, and the assumption of parallel flow, we may write down the two-dimensional vorticity equation in the form,

$$
\left\{\frac{\partial}{\partial t}+U \frac{\partial}{\partial x_{1}}-v \nabla^{2}\right\} \omega_{3}+u_{2} \Omega_{3}=-\frac{\partial}{\partial x_{i}}\left(u_{i} w_{3}-\overline{u_{i} w_{3}}\right),
$$

where we have introduced the fluctuating component of vorticity

$$
\omega_{3}=\frac{\partial u_{2}}{\partial x_{1}}-\frac{\partial u_{1}}{\partial x_{2}}
$$

and the mean vorticity vector $\Omega_{3^{\circ}}$ For two dimensional parallel mean flow we have $\Omega_{1}=\Omega_{2}=0$, and for two dimensional disturbances we have $\omega_{1}=\omega_{2}=0$.

Let us introduce a fluctuating stream function $\psi$ defined by, 


$$
u_{1}=\frac{\partial \psi}{\partial x_{2}} \quad \text { and } \quad u_{2}=-\frac{\partial \psi}{\partial x_{1}}
$$

Then the fluctuating component of vorticity $\omega_{3}$ is given by,

$$
\omega_{3}=-\nabla^{2} \psi
$$

For two dimensional parallel mean flow the mean vorticity vector $\Omega_{3}$ is given by

$$
\Omega_{3}=-U^{\prime}
$$

Equation (6.1) written in terms of the fluctuating stream function is then,

$$
\begin{aligned}
\left(\frac{\partial}{\partial t}+\frac{U \partial}{\partial x_{1}}-U \nabla^{2}\right) \nabla^{2} \psi-U^{\prime} \cdot \frac{\partial \psi}{\partial x_{1}}= & -\frac{\partial}{\partial x_{1}}\left(\nabla^{2} \psi \frac{\partial \psi}{\partial x_{2}}-\overline{\nabla^{2} \psi \frac{\partial \psi}{\partial x_{2}}}\right) \\
& +\frac{\partial}{\partial x_{2}}\left(\nabla^{2} \psi \frac{\partial \psi}{\partial x_{1}}-\overline{\nabla^{2} \psi \frac{\partial \psi}{\partial x_{1}}}\right)
\end{aligned}
$$

Equation (6.6) is a fourth order partial differential equation for the fluctuating stream function $\psi$. The boundary conditions on the velocity fluctuations are

$$
u_{1}=u_{2}=0 \text { when } u_{2} \rightarrow \overline{+}^{\infty}
$$

The boundary conditions on the stream function may be more clearly defined later, however, making use of equations (6.3) we have,

and

$$
\begin{array}{cc}
\frac{\partial \psi}{\partial x_{2}}=0 \text { as } x_{2} \rightarrow \pm \infty & \text { ) } \\
\psi=\psi\left(x_{2}\right) \text { as } x_{2} \rightarrow \pm \infty & \text { ) }
\end{array}
$$


Let us introduce the Fourier transform of the fluctuating stream function of the form,

$$
\psi\left(x_{2} ; k_{1}, \omega\right)=\frac{1}{(2 \pi)^{2}} \int_{-\infty}^{\infty} \int_{-\infty}^{\infty} e^{-i\left(k_{1} x_{1}-\omega t\right)} \psi\left(x_{2}, x_{1}, t\right) d x_{1} d t(6.9)
$$

where $\dot{\psi}$ is the Fourier transform of $\psi, k_{1}$ is the wave number in the $x_{1}$-direction and $\omega$ is the frequency. In order to assure that the above integral exists we will assume that the iluctuating vorticity, $\omega_{3}$, is zero outside a region defined by $x_{1}= \pm X_{1}$ and $t= \pm$. Then $\psi=0$ outside this region.

The Fourier transform of equation $(6.6)$ is

$$
\begin{aligned}
& (U-c)\left(\hat{\psi}^{\prime \prime}-k_{l}^{2} \hat{\psi}\right)-U^{\prime} \hat{\psi}-\frac{v}{i k_{I}}\left[\hat{\psi}^{i v}-2 k_{1}{ }^{\hat{\psi}} \dot{\psi}+k_{1}{ }^{4} \hat{\psi}\right] \\
& =-\frac{1}{k_{1}} \int_{-\infty}^{\infty} \int_{-\infty}^{\infty} k_{I}\left(\hat{\psi}^{\prime} \cdot-k_{I}^{2 a} \psi \hat{\psi}\left(k_{I}-k_{I}, w-w\right) d k_{I} d w\right.
\end{aligned}
$$

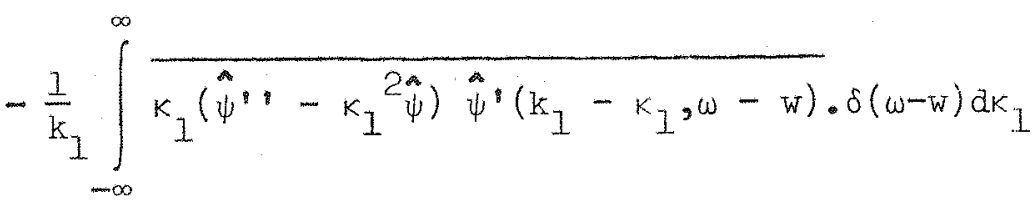

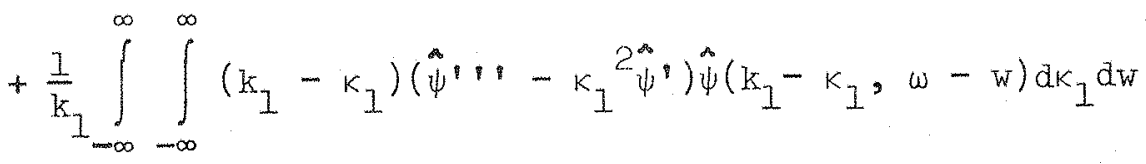

$$
\begin{aligned}
& +\frac{1}{k_{I}} \int_{-\infty}^{\infty}\left(k_{I}-k_{I}\right)\left(\hat{\psi}^{\prime} \cdot-k_{I}^{2} \hat{\psi}^{\prime}\right) \hat{\psi}\left(k_{I}-k_{I}, \omega-w\right) \delta(\omega-w) d k_{I}
\end{aligned}
$$

Following Sharma (1968) we then obtain,

$$
(U-c)\left(\hat{\psi}^{\prime}-k_{1} \hat{\psi}\right)-U^{\prime} \hat{\psi}-\frac{v}{i k_{I}}\left[\hat{\psi}^{i v}-2 k_{1}^{2} \hat{\psi}^{\prime \prime}+k_{1}{ }^{4} \hat{\psi}\right]
$$




$$
\begin{aligned}
& =\frac{1}{k_{I}} \int_{-\infty}^{\infty} \int_{-\infty}^{\infty} \hat{\psi} \cdots\left(k_{I}-k_{I}, w-w\right) k_{I} \hat{\psi}\left(k_{I}, w\right)- \\
& \left.k_{I} \hat{\psi}\left(k_{I}, w\right) \hat{\psi}^{3}\left(k_{I}-k_{I}, \omega-w\right)\right] d k_{I} d w \\
& +\frac{1}{k_{1}} \int_{-\infty}^{\infty} \frac{}{\left[\hat{\psi}^{\prime} \cdots\left(k_{1}-k, \omega-w\right) k_{1} \hat{\psi}\left(k_{I}, w\right)-\right.} \\
& \overline{\left.k_{I} \hat{\psi^{\prime}}(k, w) \hat{\psi}\left(k_{I}-k_{I}, w-w\right)\right]} \delta(w-w) d k_{I} \\
& =\int_{-\infty}^{\infty} \int_{-\infty}^{\infty} \hat{\psi^{\prime}} \cdots\left(k_{1}, w\right) \hat{\psi}\left(k_{1}-k_{I}, w-w\right) d k_{I} d w \\
& +\int_{-\infty}^{\infty} \overline{\hat{\psi}^{\prime \prime \prime}\left(k_{1}, w\right) \hat{\psi}\left(k_{1}-k_{1}, \omega-w\right)} \delta(\omega-w) d k_{I} \\
& -\frac{1}{k_{1}} \int_{-\infty}^{\infty} \int_{-\infty}^{\infty} k_{1} \frac{d}{d x_{2}} \hat{\psi}^{\prime \prime}\left(k_{1}, w\right) \hat{\psi}\left(k_{1}-k_{1}, w-w\right) d k_{1} d w-
\end{aligned}
$$

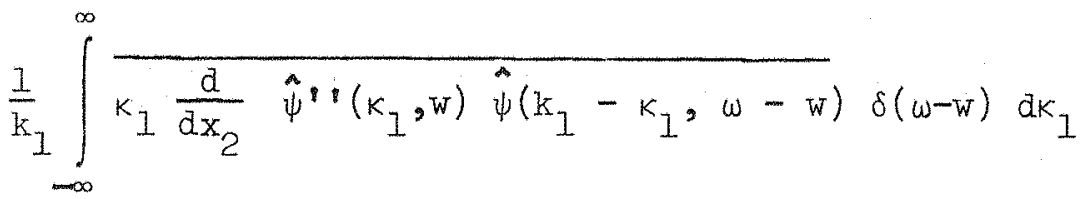

$$
\begin{aligned}
& =-\frac{1}{k_{1}} \int_{-\infty}^{\infty} \int_{-\infty}^{\infty}\left(k_{1}-k_{I}\right)\left(\hat{\psi}^{\prime} \cdot\left(k_{1}-k_{I}, \omega-w\right)-\left(k_{1}-k_{1}\right)^{2}\right. \\
& \left.\hat{\psi}\left(k_{I}-k_{I}, w-w\right)\right) \hat{\psi}^{0}\left(k_{I}, w\right) d k_{I} d w
\end{aligned}
$$

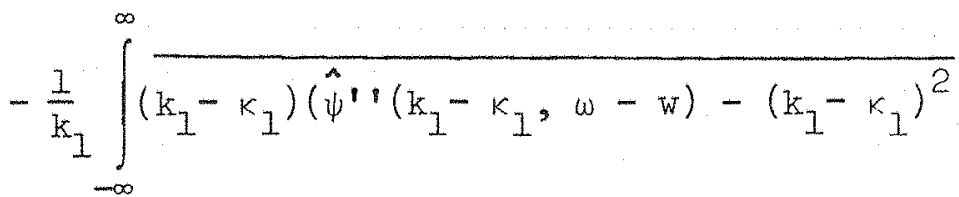

$$
\begin{aligned}
& \left.\hat{\psi}\left(k_{I}-\kappa_{I}, \omega-w\right)\right) \hat{\psi}^{\prime}\left(\kappa_{I}, w\right) \delta(\omega-w) d \kappa_{I} \\
& -129-
\end{aligned}
$$




$$
\begin{aligned}
& +\frac{1}{k_{I}} \int_{-\infty}^{\infty} \int_{-\infty}^{\infty} k_{I}\left(\psi^{n}:\left(k_{I}-k_{I}, \omega-w\right)-\left(k_{I}-k_{I}\right)^{2} \cdot \hat{\psi}^{8}\left(k_{I}-k_{I},\right.\right. \\
& w-w)) \psi\left(k_{1}, w\right) d k_{1} d w
\end{aligned}
$$

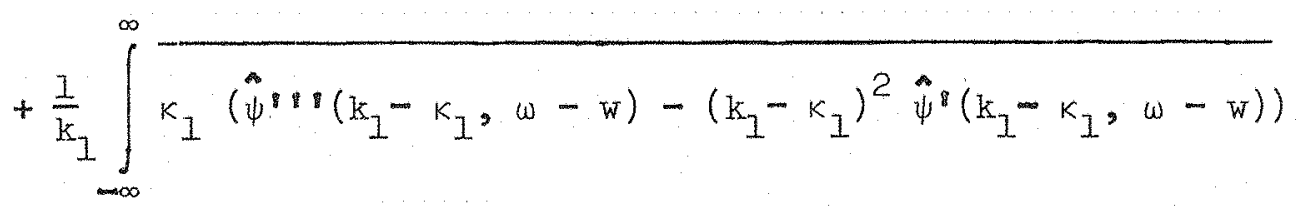

$$
\begin{aligned}
& \overline{\psi\left(\kappa_{1}, w\right)} \delta(\omega-w) d \kappa_{1} \\
& =-\int_{-\infty}^{\infty} \int_{-\infty}^{\infty} \hat{\psi}^{\prime}\left(k_{I}, w\right)\left(\hat{\psi}^{\prime} \cdot\left(k_{I}-k_{I}, w-w\right)-\left(k_{I}-k_{I}\right)^{2} \hat{\psi}\left(k_{I}-k_{I}\right.\right. \\
& w-w)) d k_{I} d w \\
& +\frac{1}{k_{1}} \int_{-\infty}^{\infty} \int_{-\infty}^{\infty} k_{1} \hat{\psi}\left(k_{1}, w\right)\left(\hat{\psi}^{2}\left(k_{1}-k_{1}, w-w\right)-\left(k_{1}-k_{I}\right)^{2}\right. \\
& \left.\hat{\psi}\left(k_{I}-\kappa_{I}, w-w\right)\right) d \kappa_{I} d w \\
& +\frac{1}{k_{1}} \int_{-\infty}^{\infty} \int_{-\infty}^{\infty} k_{I} \hat{\psi}\left(k_{I}, w\right)\left(\hat{\psi} \cdot\left(k_{1}-k_{1}, w-w\right)-\left(k_{1}-k_{1}\right)^{2}\right. \\
& \left.\hat{\psi}^{\prime}\left(k_{1}-k_{1}, w-w\right)\right) d k_{1} d w \\
& +(
\end{aligned}
$$

where ( ) denotes the time average of the preceding terms. The delta functions arise in the time averaged integrals as these then lead to the Fourier transforms of constants. 
Let us consider the right hand side of equation (6.10). The integrand of the first integral is the product of the $u_{1}-$ fluctuation and the vorticity fluctuation. The integrand of the second integral corresponds to the product of the vorticity fluctuation and the derivative with respect to $x_{1}$ of the $u_{1}-$ fluctuation. The final integrand represents the product of the gradient of the vorticity fluctuation and the $u_{2}$-fluctuation. We also notice that if the right hand side of equation (6.10) is set equal to zero the homogeneous equation is the well know Orr-Sommerfeld equation we have previously derived in our consideration of the stability of the mixing layer.

The solution of equation $(6.10)$ in the form written is a formidable task, the difficulty lying in the presence of the non-linear terms on the rightmhand side. We will discuss the various previous treatments of the non-linear problem when we introduce the problem of finite amplitude disturbances in the next chapter. However, we will introduce a number of those ideas here as an explanation in part of our method of solution for the free shear layer.

A method of solution involving a perturbation of the mean profile was used by Sharma (1968) in his work on the boundary layer. If we assume that the vorticity fluctuations in equation (6.10) may be taken out from the integrals then the velocity fluctuations and derivatives alone remain in the integrands. Then the integrals may be evaluated and combined with the terms on the left hand side of equation $(6.10)$. This can be shown 
to be equivalent to perturbing the mean velocity profileo Let us returm to our two-dimensional model. The vorticity equation is,

$$
\begin{aligned}
& \left.\left(\frac{\partial}{\partial t}+U \frac{\partial}{\partial x_{1}}-v \nabla^{2}\right) \omega_{3}-u_{2} \frac{\partial^{2} U}{\partial x_{2}^{2}}=-\frac{\partial}{\partial x_{i}}\left(u_{i} \omega_{3}-\overline{u_{i} \omega_{3}}\right)\right) \\
& =-\left(u_{1} \frac{\partial \omega_{3}}{\partial x_{1}}+u_{2} \frac{\partial \omega_{3}}{\partial x_{2}}\right)+\left(\overline{u_{1} \frac{\partial \omega_{3}}{\partial x_{1}}+u_{2} \frac{\partial \omega_{3}}{\partial x_{2}}}\right)
\end{aligned}
$$

We may rewrite this equation as,

$$
\begin{array}{rll}
\left(\frac{\partial}{\partial t}+\left(U+u_{1}\right) \frac{\partial}{\partial x_{1}}-v \nabla^{2}\right) \omega_{3}-u_{2}\left(\frac{\partial^{2} U}{\partial x_{2}{ }^{2}}-\frac{\partial \omega_{3}}{\partial x_{2}}\right) & ) & \\
& =\left(u_{1} \frac{\partial \omega_{3}}{\partial x_{1}}+u_{2} \frac{\partial \omega_{3}}{\partial x_{2}}\right) & )
\end{array} \quad \text { (6.12) }
$$

Writing this equation in terms of the stream function we have,

$$
\begin{aligned}
&\left.\left(\frac{\partial}{\partial t}+\left(u+u_{1}\right) \frac{\partial}{\partial x_{1}}-\nu \nabla^{2}\right) \nabla^{2} \psi-\frac{\partial \psi}{\partial x_{1}}\left(\frac{\partial^{2} u}{\partial x_{2}^{2}}+\frac{\partial^{2} u_{1}}{\partial x_{2}^{2}}-\frac{\partial^{2} u_{2}}{\partial x_{1} \partial x_{2}}\right)\right) \\
&=-\left(\frac{\partial u_{1} \omega_{3}}{\partial x_{1}}+\frac{\partial u_{2} \omega_{3}}{\partial x_{2}}\right))
\end{aligned}
$$

The right hand side of equation (6.13) represents the timeaveraged convection of vorticity by the turbulent fluctuations. The relative magnitudes of these time averaged terms may be judged by assuming that the vorticity is a transferable quantity and may be expressed as,

$$
\overline{u_{2} \omega_{3}}=-\overline{\left(u_{2}^{2}\right)^{\frac{1}{2}}} \quad L_{2} \frac{d^{2} u}{d x_{2}^{2}},
$$




$$
\overline{u_{1} w_{3}}=-\left(\bar{u}_{1}^{2}\right)^{\frac{1}{2}} L_{1} \frac{a^{2} u}{d x_{2}^{2}}
$$

where $L_{1}$ and $L_{2}$ are length scales. The values of $\left(\overline{u_{2}^{2}}\right)^{\frac{1}{2}}$ may be considered small at the points of maximum $\frac{d^{2} U}{d x_{2}{ }^{2}}$ and the gradient of $\overline{u_{2} \omega_{3}}$ may be considered much smaller than ${ }^{2}$ the gradient of $u_{2} w_{3}$. The variation of $\left(u_{1}^{2}\right)^{\frac{1}{2}}$ with $x_{1}$ is small for parallel or nearly parallel flows and the variation of $\overline{u_{1} w_{3}}$ with $x_{1}$ can be neglected in comparison with the gradient of $u_{1} 3^{\circ}$ Thus we may neglect the terms on the right hand side of equation (6.13) in comparison to the terms representing the convection of vorticity by the turbulence on the left hand side. The remaining homogeneous equation is still too complicated for useful solution, Sharma solved the homogeneous equation by assuming that the solution to the linear problem, that is the problem neglecting all non-linear terms, was the first approximation to the general solution. He was then able to modify the mean profile to obtain the next approximation. If we write

$$
\tilde{U}=U+u_{I}
$$

where $u_{1}$ is the solution of the linear homogeneous equation then the second approximation is a solution of the equation,

$$
\left(\frac{\partial}{\partial t}+\tilde{U} \frac{\partial}{\partial x_{1}}-\nu \nabla^{2}\right) \nabla^{2} \psi-\frac{\partial \psi}{\partial x_{1}}\left(\frac{\partial^{2} \tilde{U}}{\partial x_{2}^{2}}\right)=0
$$

The results obtained by Sharma for the first iteration of the modified mean profile led to enhanced amplification. He showed that a perturbation in the mean profile excited large 
scale motions in the boundary layer. In the boundary layer case, however, the disturbances are either stable, neutral or only very slightly amplified. The inclusion of the perturbation on the mean profile leads clearly to an inflexional instability on the outer part of the boundary layer. This inflexional instability leads to much higher values of amplification. Thus we see that considered in this way the non-linear terms have acted as a destabilising influence。 However, we must consider that the non-linear terms have a stabilising effect as well for it is known that there is a limiting value to the turbulence intensity. Let us consider that the background turbulence may be associated with an eddy viscosity term. We must be clear here that this eddy viscosity 'may' or may not take the same value as that used in our mean flow analysis (Chapter 3). The eddy viscosity used there was contributed to by the large scale turbulent motions. We are here dealing with a turbulent eddy viscosity associated with the small scale background turbulence. We may write the eddy viscosity such that,

$$
T_{i}=-\frac{\partial}{\partial x_{j}} v_{T}\left(\frac{\partial u_{i}}{\partial x_{j}}+\frac{\partial u_{j}}{\partial x_{i}}\right)
$$

where $T_{i}$ is the Reynolds stress function defined by,

$$
T_{i}=\frac{\partial}{\partial x_{j}}\left(\overline{u_{i} u_{j}}-u_{i} u_{j}\right)
$$

Then the new form of the transformed equation (6.10) is

$$
\begin{aligned}
& (U-c)\left(\hat{\psi}^{B}-\mathrm{k}^{2} \dot{\psi}\right)-\mathrm{U}^{\beta} \dot{\psi} \\
& =-\frac{i}{k}\left(\frac{1}{R}+\frac{1}{R_{T}}\right)\left(\hat{\psi}^{i v}-2 k^{2} \hat{\psi}^{\theta}+k^{4 a} \psi-\frac{2}{k} i\left(\hat{\psi}^{*} \cdot-k^{2} \psi^{8}\right) \cdot \frac{d \nu_{T}}{d x_{2}}\right)
\end{aligned}
$$




$$
\left.-\frac{i}{k} \cdot\left(\psi^{\infty}-k^{2} \psi\right) \frac{d^{2} v_{T}}{d x_{2}^{2}}\right)
$$

We have seen from our calculations for the mean velocity profile that the assumption of a constant eddy viscosity distribution does lead to a representation not unlike the measured values: Thus we may ascribe a constant value to $\nu_{T}$ to obtain a reprem sentation of the effect of its introduction on the equation s solution. With $\nu_{T}$ constant the equation (6.17) leads to the homogeneous Orr-Sommerfeld equation with the kinematic viscosity enhanced by an eddy viscosity。 This leads to a net effect of a reduction in the effective Reynolds number. As we have seen from our calculation of the stability of a mixing layer a reduction in Reynolds number has the effect of stabilising the flow. Though we have performed no calculations using an eddy viscosity it does not seem unreasonable to tentatively suggest the dual role of the turbulence in the flow. We have seen from equation (6.14) that a perturbation in the instantaneous velocity profile has a destabilising effect whereas as the turbulence level increases the formation of an eday viscosity has a stabilising or amplitude limiting effect.

We have noticed however that the role of the turbulence in enhancing the growth of disturbances, by modifying the mean velocity profile, was chiefly successful in Sharma"s work. for the case of the boundary layex, owing to the introduction of an inflection point in the mean profile. The boundary layer does not have an inflexion point in the mean profile, 
except for special cases such as at the separation point. Thus it is clear that in this case modification of the mean profile will have a dominating effect on the growth of disturbances. However, we must question whether the effect would be as great if the same formulation of the problem were used in the case of the mixing layer between two streams. In this case there already exists a strong inflexional instability in the mean velocity profile which leads to strong amplification of disturbances. Thus we may argue that the former role of the turbulence expressed above as a destabilising influence will not be best considered by perturbing the mean velocity profile. That is to say that for the case of the mixing layer the first approximation, the linear solution, to the non-linear equation (6.14) will not be modified greatly by a perturbation of the mean velocity profile. In view of this a different formulation of the non-Iinear problem for the case of the mixing layer has been used and this is presented in the next chapter. However we must expect that our first linear approximation will adequately represent the fluctuations in the turbulent mixing layer.

From the preceding discussion it would appear that the solutions to the homogeneous linear equation plays an important role in the description of fluctuations in the turbulent mixing layer. Eckhaus (1965) expanded the solution to the non-linear problem in terms of solutions to the linearised equation. If the solution to the non-linear problem is given by $\Phi^{\prime}\left(x_{i}, t\right)$ then at any instant of time it may be represented 
by the series expansion

$$
\Psi^{\prime}\left(x_{i}, t\right)=\sum_{m=0}^{\infty} A_{m}(t) \Psi_{m}\left(x_{i}\right)
$$

where $\Psi_{m}\left(x_{i}\right)$ are the solutions of the linearised problem and $A_{m}(t)$ are 'amplitude functions' which may be determined from the relation,

$$
A_{m}=\frac{1}{\left(c-c_{m}\right)} \int_{-\infty}^{\infty} f\left(x_{2}\right) \Psi_{m}\left(x_{2}\right) d x_{2}
$$

where $c_{m}$ represents the complex eigenvalues for given values of $k_{1}$ and $R$ in the solution of the linearised homogeneous equation (6.10), $f\left(x_{2}\right)$ represents the non-linear right hand side of this equation and $\hat{\Psi}_{m}\left(x_{2}\right)$ are the corresponding eigenfunctions of the adjoint problem given by,

$$
\begin{aligned}
& \left.(\mathrm{u}-\mathrm{c})\left(\hat{\psi}^{\prime}-\mathrm{k}_{1}^{2} \hat{\psi}\right)+2 \mathrm{U} \hat{\psi}^{\prime}+\frac{\dot{i} v}{\mathrm{k}_{1}}\left(\hat{\psi}^{i v}-2 \mathrm{k}_{1}^{2} \tilde{\psi} \cdot+\mathrm{k}_{1} \dot{\psi} \psi\right)=0\right) \\
& \text { with } \tilde{\psi}(+\infty)=\tilde{\psi}(-\infty)=\hat{\psi}^{\prime}(+\infty)=\tilde{\psi}^{\prime}(-\infty)=0
\end{aligned}
$$

In equation (6.19) the adjoint eigenfunction and non-linear terms are functions of $x_{2}$ only. In the preceding analysis we have introduced the concept that for given values of $k_{l}$ and $P_{1}$ there exists no unique eigenvalue c. In stability theory we seek the lowest eigenvalue of the sequence. Eckhaus, interpreting the results of Corcos and Sellars (1959), stated that 'in the first asymptotic approximation for $R \rightarrow \infty$ the number of eigenvalues is found to be finite for every fixed value of $R$ 
and increasing without limit as $R \rightarrow \infty$ ' In chapter 4 we discussed methods of solution of the Orr-Sommerfeld equation which involved variational techniques. It is clear that in this case the solution of the matrix problem will give rise to a sequence of eigenvalues. Thus numerically we may evaluate the eigenvalue sequences.

In our approach to the solution of this problem we will make use of the first term in the eigenfunction analysis, that is the solution involving the lowest eigenvalue. A similar approach has been used by Landahl (1967) in his wave-guide model for the turbulent boundary layer. In his formulation he sought the lowest eigenvalue to the Orr-Sommerfeld problem. However it is thought probable that his failure to find any amplifying modes to the boundary layer problem was due to the inaccuracies in his purification scheme for the numerical solution at high Reynolds numbers. Landahl considered that the cross-spectral density of the pressure fluctuations will be dominated by lightly damped waves. This can be seen as these waves will correlate over a larger distance. However in the case of the free shear layer there exists strong amplification and it does not seem reasonable to describe the structure of the flow in terms of lightly damped or neutral disturbances. We have already seen in our discussion of the stability of free shear layers that the most amplifying mode dominates the local flow structure. This is clearly seen in the work of Michalke (1965). Thus we propose to describe the structure of the turbulent flow in terms of the most amplifying eigen- 
function solution of the homogeneous equation,

$$
(U-c)\left(\hat{\psi}^{\prime}-k_{I}{ }^{2} \hat{\psi}\right)-U^{\prime} \hat{\psi}+\frac{i v}{k_{I}}\left(\hat{\psi}^{i v}-2 k_{I}^{2} \hat{\psi}^{\prime}+k_{I}{ }^{4} \hat{\psi}\right)=0
$$

In the light of the correspondence between theory and measurements in stability work on the free mixing layer using spatial amplification we will consider the spatial mode in our calculations. That is we will consider a real frequency and complex wavenumbers and eigenvalues.

In the next section we will describe the method of solution of equation (6.21) and the numerical values of parameters chosen to correspond with experiment. We will later discuss how the introduction of the most amplifying eigenfunction is compatible with our previous discussion of amplitude limitation.

\subsection{Numerical Calculation Procedure for the Solution of the Homogeneous Equation of Turbulent Shear Flow}

Let us first non-dimensionalise equation (6.21) with respect to a reference length $I_{0}^{*}$, and a reference velocity $U_{0}^{*}$ Equation (6.21) then yields,

$$
(U-c)\left(\hat{\psi}^{\prime} \cdot-\alpha^{2} \hat{\psi}\right)-U^{\prime} \hat{\psi}+\frac{i}{\alpha R}\left(\hat{\psi}^{i v}-2 \alpha^{2} \hat{\psi}^{\prime}+\alpha^{4} \hat{\psi}\right)=0
$$

where $\alpha=k_{I} L_{0} *, R=\frac{U_{0}{ }^{*} L_{0}{ }^{*}}{\nu^{*}}$ and all other quantities in equation (6.22) are non-dimensional. Let us write the mean velocity in the form, as in Chapter 3,

$$
U^{*}=\frac{U_{1}^{*}+U_{2}^{*}}{2}+\frac{U_{1}^{*}-U_{2}^{*}}{2} f^{\prime}(y / \theta)
$$

where $\theta$ is the momentum thickness and dimensional quantities are starred, Then letting $\mathrm{U}_{0}^{*}=\frac{\mathrm{U}_{1}{ }^{*}-\mathrm{U}_{2}^{*}}{2}$ and referring all 
lengths to $\theta$ we obtain from $(6.22)$,

$$
\begin{gathered}
\left.\left(f^{\prime}-c^{*}\right)\left(\hat{\psi}^{\prime}-\alpha^{2} \hat{\psi}\right)-f^{\prime \prime} \hat{\psi}+\frac{i}{\alpha R}\left(\hat{\psi}^{i v}-2 \alpha^{2} \hat{\psi}^{\prime \prime}+\alpha^{4} \hat{\psi}\right)=0,\right) \\
\text { where } c^{*}=c-\left(\frac{U_{1}+U_{2}}{2}\right)
\end{gathered}
$$

The detailed solution of equation $(6.24)$ has been dealt with in Chapter 5 and Appendix II, however we will describe again here the main features.

Above the mixing layer $U^{*}=U_{2}$ and $f^{\prime}=1$. The solutions to the equation are then

$$
\ddot{\psi}=\sum_{n=1}^{4} A_{n} e^{p_{n} y / \theta},
$$

$$
\begin{array}{ll}
\text { where } \quad p_{1}=\alpha, & p_{2}=-\alpha \\
p_{3}=\left(\alpha^{2}+i \alpha R\left(I-c^{*}\right)\right)^{\frac{1}{2}}, & \left.p_{4}=-\left(\alpha^{2}+i \alpha R\left(I-c^{*}\right)\right)^{\frac{1}{2}}\right)
\end{array}
$$

Below the shear layer the solutions are of the form,

$$
\hat{\psi}=\sum_{n=1}^{4} B_{n} e^{q_{n} y / \theta},
$$

$$
\begin{array}{ll}
\text { where } \quad q_{1}=\alpha, \quad q_{2}=-\alpha, & q^{2} \\
q_{3}=\left(\alpha^{2}-i \alpha R\left(1+c^{*}\right)\right)^{\frac{1}{2}}, & \left.q_{4}=-\left(\alpha^{2}-i \alpha R\left(1+c^{*}\right)\right)^{\frac{1}{2}}\right)
\end{array}
$$

The computation is started with $A_{2}=1$ and $A_{4}=0$ and the integration is performed from above to below the layer. At some point below the layer we may evaluate the values of $B_{n}$ 
for this solution. The quantities $B_{n}$ may be obtained by inverting the equation (6.27) which Ieads to,

$$
\begin{aligned}
& B_{I}=\frac{-e^{-\alpha y / \theta}}{2 a\left(q_{3}^{2}-\alpha^{2}\right)}\left(\hat{\psi} \cdots+\alpha \hat{\psi} \cdot+q_{3}{ }^{2} \hat{\psi}^{\prime}-\alpha q_{3}{ }^{2} \hat{\psi}\right), \\
& \alpha y / \theta \\
& B_{2}=\frac{e}{2 \alpha\left(q_{3}^{2}-\alpha^{2}\right)}\left(\hat{\psi}^{\prime} \cdots-\alpha \hat{\psi}^{\prime}+q_{3}^{2} \hat{\psi}^{\prime}+\alpha q_{3}^{2} \hat{\psi}\right), \\
& B_{3}=\frac{e^{-q_{3} y / \theta}}{2 q_{3}\left(q_{3}^{2}-\alpha^{2}\right)}\left(\hat{\psi}^{\prime} \dot{\psi}+q_{3} \hat{\psi}^{\prime \prime}-\alpha^{2} \hat{\psi}^{\prime}-\alpha^{2} q_{3} \hat{\psi}\right) \text { and } \\
& B_{4}=\frac{-e^{q_{3} y / \theta}}{2 q_{3}\left(q_{3}^{2}-\alpha^{2}\right)}\left(\hat{\psi}^{\prime} \cdot-q_{3} \hat{\psi}^{\prime} \cdot-\alpha^{2} \hat{\psi}+\alpha^{2} q_{3} \hat{\psi}\right) .
\end{aligned}
$$

The values of the constants $B_{n}$ evaluated using the values of the computations with $A_{2}=1$ and $A_{4}=0$ we will call $B_{n_{I}} \cdot A$ second integration is then performed with $A_{2}=0$ and $A_{4}=1$ as the starting conditions and the corresponding values of $B_{n}$ wre called $B_{n_{I I}}$. As the equation is Iinear we have that for any arbitrary values of $A_{2}$ and $A_{4}$ the values of $B_{n}$ are given by $B_{n}=A_{2} B_{n_{I}}+A_{4} B_{n_{I I}}$. We require that for a bounded solution below the mixing layer $B_{2}=B_{4}=0$. We may find the value of $A_{4}$ for which $B_{2}=0$ for $A_{2}$ set arbitrarily equal to unity from the above equation. The equation for $\mathrm{B}_{4}$ is then

$$
B_{4}=B_{4}-\frac{{ }^{B_{2}}}{B^{2} I I} \cdot{ }^{B_{4} I}
$$

Equation (6.29) leads to the numerical method for calculating the eigenvalue for fixed values of $\omega$ and $R_{0}$ for any value 
of $\omega$ and $R$ the value of $c^{*}$ is altered such that the value of $B_{4}$ is set as near zero as possible. Further details of the numerical purification scheme needed for large Reynolds numbers and the interpolation procedure may be found in chapter 5 and Appendix II.

In our calculations we will consider the problem of a mixing layer for which $U_{2} / U_{1}=0.61$. This corresponds to the experiments of Yule (1970). The value of $R$ used is 6,000, which corresponds to a reference velocity $\left(U_{1}-U_{2}\right) / 2$ of $3.55 \mathrm{~m} / \mathrm{sec}$ and a reference thickness of $10^{-2} \mathrm{M}$ 。

In the next section we will describe the results of our calculations for the mixing layer between two streams. The results will be compared, where possible, with the experimental results of Yule.

\subsection{Discussion of Results}

The mean velocity profile used in the numerical calculations was found theoretically by the method of solution given in Chapter 3. We saw there that this gave excellent agreement with the experimental profile. A numerical solution was used, rather than the experimental values, for the mean velocity profile as it was found to be difficult to obtain a smooth second derivative from the measured values. However for the case of the mixing layer neither the mean velocity nor its second derivative have very high values, as is the case for the boundary layer, and it is unlikely that very small differences between theoretical and measured profiles will greatly affect the calculations. 
We will now present the results of our calculations for the turbulent mixing layer. In a number of cases quantities which are predicted theoretically have not been measured by Yule for this particular velocity ratio of 0.61 . In this case we will attempt to relate certain experimental results for different velocity ratios.

\subsubsection{The phase velocity and the convection velocity}

The phase velocity is shown in Figures 31 for both cases of spatial and temporal amplification respectively. Although we will concentrate most of our succeeding discussion on the spatial case it is thought useful to include both here for comparison. It is clear that the phase velocity is a function of frequency. In his calculations for the boundary layer Sharma (1968) found that as the frequency increased so the phase velocity becomes independent of the frequency. In our calculations for the mixing layer this is not the case. In the instance of spatial amplification the phase velocity has a peak value at low frequency then decreases to a minimum with increasing frequency and finally begins to increase with frequency. For temporal amplification the phase velocity is increasing monotonically with frequency with a large range of near independence of frequency in the middle wavenumber regions. If we recall the results of the stability analysis of Chapter 5 we noticed that at low frequencies the calculations were independent of the mean profile shape and at high frequencies the profile shape was a critical factor. Thus it 
is clear that our particular choice of velocity profile accounts for the rise in phase velocity at the higher frequencies. The range of variation of phase velocity in the spatial case is between $49 \%$ and $58 \%$ of the velocity difference between the two free streams. The experimental results of Davies and Bose (1968) show that the phase velocity is nearly independent of frequency and takes a value approximately equal to the local mean velocity. We must note that measurements of convection velocity depend on the transverse position of the measuring instrument in the mixing layer. In our calculations the phase velocity predicted is not a function of the transverse co-ordinate. It would appear reasonable that our predicted phase velocity should thus lie close to the arithmetic mean of the velocities of the two streams. If we corsider the relationship between spatial and temporal variations we may write,

$$
\frac{\partial}{\partial t}=-U_{c} \frac{\partial}{\partial x}
$$

where $U_{c}$ is the convection velocity. If we Fourier transform this expression we see that,

$$
U_{c} \propto c_{r} \quad,
$$

where $c_{r}$ is the phase velocity. As we have previously discussed in considering the relationship between temporally and spatially growing disturbances, the constant of proportionality in equation (6.31) will be a function of the group velocity, $c_{g}=\frac{\partial \omega_{r}}{\partial \alpha_{r}}$. The variation of phase velocity with frequency for the range of 
frequencies considered is not great though there exists no region of frequency independence. However the error involved in equating $U_{C}$ and $c_{r}$ in equation (6.31) is unlikely to be large. The measurements of Davies, Fisher and Barrett (1963) confirm that the difference between convection velocity and phase velocity in the mixing region of a jet is never greater than $10 \%$. It is interesting to note that the phase velocity in the spatial case reaches a maximum value at low frequencies. In the case of $U_{2}$ $=0$ the calculations of Michalke (1965) for the stability problem, indicate that the phase velocity increases with decreasing frequency and reaches a value equal to the velocity $U_{1}$ at zero frequency. The phase velocity, as we have noted above, is related to the convection of eddies in the flow. If we consider the spatial case it is clear that the large eddies move faster than the smaller although there is an increase in velocity towards the higher frequencies。

The amplification factor, $\alpha_{i}$, in the spatial case is plotted as a function of frequency in Figure 3 . The amplification is strong as is to be expected for the profile of a mixing layer with an inflection point. The maximum value of amplification factor is -.073699 and occurs at a frequency, $\omega$, of 2.50275. The phase velocity of the maximum amplifying mode is 4.07343 which is $50.15 \%$ of the velocity difference of the two streams above the value of $\mathrm{U}_{2}$.

\subsubsection{Rate of growth or decay of eddies}

The ratio $\alpha_{i} / \alpha_{r}$, in the spatial case, is related to the 
rate of decay of the eddies. The rate of growth, $-\alpha_{i} / \alpha_{r}$, is shown against frequency in Figure 33. It may be seen that the rate of growth is largest at low frequencies and the growth rate decreases with increasing frequency until the neutral frequency is reached. This suggests that the rate of growth of the large eddies is much faster than that of the small eddies. The calculations of Sharma (1968) for the boundary layer in which the amplification is very small, show that the larger edaies decay slower than the smaller ones. An extension of our curve in Figure 33 for higher frequencies at which damping takes place would show the same features as Sharma noticed assuming that the curve continued with the same sign of derivative at higher frequencies.

We have only performed calculations in this instance for one Reynolds number. However if we can assume that the results of Sharma for the boundary layer will be analogous to ours for the mixing layer it is to be expected that alteration in the magnitude of the Reynolds number will have only a small effect and then only at the higher frequencies. We may explain this by noting that the larger eddies will be dominated by inertial forces and will be little affected by viscous forces.

\subsubsection{The fluctuating velocity and pressure field}

In this section we will examine the distributions of fluctuating velocity in the mixing layer by a suitable use of the eigensolutions to equation (6.22). A complete description of the fluctuating velocity field would be obtained by 
applying the inverse Fourier transform to the stream function in equation (6.22). This would involve an integration over wavenumber and frequency. However, as we have argued above, the flow structure at any axial position in the mixing layer will be dominated by the mode receiving maximum amplification. The eigensolution to equation (6.22) which receives maximum amplification is shown in Figure $34(a)$ and (b). The solution has been normalised such that the real part of $\hat{\psi}$ is unity at $y / \theta=0$ and the imaginary part of $\hat{\psi}$ is zero. The relationships between $\hat{u}_{2}$ and $\hat{\psi}$ and $\hat{u}_{1}$ and $\hat{\psi}$ are given by the transformed form of equation (6.3); they are

$$
\begin{array}{ll}
\hat{u}_{2}=-i \alpha \dot{\psi} \text { and } & \text { ) } \\
\hat{u}_{1}=\hat{\psi}^{\prime} & \text { ) }
\end{array}
$$

The derivative of $\hat{\psi}$, which is related to $\hat{u}_{1}$ through equation (6.32) is shown in Figures $35(a)$ and (b). The change of sign and relative magnitudes of real and imaginary parts of $\hat{\psi}$ and $\psi^{*}$ makes description of the distributions of velocity fluctuations difficult. We will therefore conside the modulii of squared $\stackrel{A}{u}_{1}$ and $\hat{u}_{2}$. These are shown in Figures 36 and 37 respectively. The results have been normalised in both instances such that the modulus of $\hat{u}_{1}$ is unity when $y / \theta$ is zero. Also shown on Figure 36 are the measurements of Yule (1970) for the mixing layex with $U_{2} / U_{1}=.61$. It may be seen that the agreement over most of the mixing layer is very good. 
The experimental results have been modified such that the maximum experimental value equals the maximum theoretical value. The measured values do not correspond at the edges of the mixing layer where the theoretical curves predict two small peaks. The values of mean velocity at the position of the two minima in the predictions are $5 \%$ and $93 \%$ of the velocity difference. The phase difference of the two smaller peaks is $180^{\circ}$ and the maximum at the centre of the layer is $90^{\circ}$ out of phase with both smaller peaks. The corresponding measured values of $\left|\hat{u}_{2}\right|^{2}$ are also shown in Figure 37. It can be seen that the measured values are twice as large as predicted values. The two peaks in the theoretical profile correspond, through the continuity equation, with the two minima of the $\left|\hat{u}_{1}\right|^{2}$ values. We may see from the results of the stability analysis of Chapter 5, or from equation (6.32), that for the magnitude of $\left|\hat{u}_{2}\right|^{2}$ to correspond to the measured values, assuming two-dimensional disturbances, the wavenumber, $\alpha$, should be of order unity. However as this does not correspond to the wavenumber for maximum amplification it would appear more likely that the low values of $\left|\hat{u}_{2}\right|^{2}$ are due to our assumption of two dimensional disturbances. The same effect was noticed by Sharma in his results for the boundary layer. No calculations have been made for oblique waves, however the effect of obliquity on the value of the normal velocity fluctuation may be seen qualitatively by considering a simple example. For high values of Reynolds number the inviscid solution is a good approximation to full equation. We will consider a shear layer where the mean velocity profile is given by 


$$
U=U_{1}^{*}+U_{2}^{*}+y / \theta, 0<y / \theta<1 .
$$

We may then write the inviscid equation for the $\hat{u}_{2}$ fluctuation,

$$
\hat{u}_{2}^{\prime \prime}=\left(\alpha^{2}+\beta^{2}\right) u_{2} \text {, }
$$

where $\beta$ is the non-dimensional wavenumber in the $x_{3}$-direction. The admissible solution of equation (6.34) for $y / \theta>0$ is,

$$
\begin{aligned}
& \hat{u}_{2} \alpha e^{-\left(\alpha^{2}+\beta^{2}\right) y / \theta} \\
& =e^{-\left(\alpha^{2} \sec ^{2} \phi\right)^{\frac{7}{2}} y / \theta},
\end{aligned}
$$

where $\phi$ is the wave angle given by $\phi=\tan ^{-1} \beta / \alpha$. We may write equation $(6.35)$,

$$
\hat{u}_{2} \alpha e^{-(\alpha / \cos \phi) y / \theta}
$$

Thus we see that the magnitude of $\hat{u}_{2}$ is dependent on the magnitude of $\cos \phi$. So the magnitude of $\hat{u}_{2}$ decreases as $\phi$ increases. This contradicts the result of Sharma who incorrectly interpreted a trigonometric relation. Thus we see that the maximum magnitude of $\hat{u}_{?}$ occurs when $\phi=0$ or for two-dimensional disturbances. However we may see from equation $(6.32)$ that the magnitude of $\hat{u}_{2}$ increases as the wavenumber increases so the magnitude of the $\hat{u}_{?}$ fluctuation will increase if averaging is performed over wavenumber instead of considering the maximun amplifying mode alone.

The distribution of Reynolas stress function is shown in Figure 38. NIso shown in the figure are the results obtained 
by Yule. The experimental results have been modified so that the maximum experimental valus corresponds to the theoretical maximum value. The distribution is very well predicted by the Iinear theory in this case. The curve exhibits a peak at a value of $y / \theta$ corresponding to the point of maximum mean shear, $U^{\prime}$. It is interesting to note that the Reynolds stress distribution exhibits no peaks or troughs which are evident in the fluctuating velocity distributions. The distribution of Reynolds stress coefficient or shear correlation coefiticient is shown in Figure 39. The distribution appears to take a constant value over most of the mixing layer. The experiments of Bradshaw et. a1. (1963) give a maximum value of $\mathrm{R}_{12}$ of .54 compared with Liepmann and Laufer of .56 . The reason for the high value of correlation may be seen by comparing the eigenfunction and its derivative. At $y / \theta=0$ the function is $\pi / 2$ radians out of phase with the derivatives. Thus, making use of equation (6.32) we see that the $\hat{u}_{1}$ and $\hat{u}_{2}$ component; are nearly in antiphase at the centre of the layer. This is true over all the mixing layer except for a small region of $y / \theta<0$. This accounts for the dip in the correlation curve.

The equation for the fluctuating pressure may be obtained by returning to our original equation of motion. The Navierm Stokes equations may be written,

$$
\frac{\partial u_{i}}{\partial t}+\frac{\partial}{\partial x_{j}} u_{i} u_{j}=-\frac{1}{\rho} \frac{\partial p}{\partial x_{i}}+v \nabla^{2} u_{i}
$$

Making use of the equation of continuity and taking the 
divergence of $(6.36)$ we obtain

$$
\nabla^{2} p\left(x_{i}, t\right)=-\rho \frac{\partial^{2}}{\partial x_{i} \partial x_{j}} u_{i} u_{j},
$$

which is the Poisson equation for the pressure. Dividing the velocity and pressure into mean and fluctuating components, substituting into equation $(6.37)$ and subtracting the mean part leads to

$$
\nabla^{2} p=-2 \rho \frac{\partial u_{2}}{\partial x_{l}} \frac{\partial U}{\partial x_{2}}-\rho \frac{\partial}{\partial x_{i} \partial x_{j}}\left(u_{i} u_{j}-\overline{u_{i} u_{j}}\right)
$$

or $\nabla^{2} p=-2 \rho \frac{\partial u_{2}}{\partial x_{1}} \frac{\partial U}{\partial x_{2}}+\rho \frac{\partial}{\partial x_{i}} T_{j}$,

where $T_{i}=\frac{\partial}{\partial x_{j}}\left(\overline{u_{i} u_{j}}-u_{i} u_{j}\right)$ is the divergence of the Reynolds stress tensor. The equations for the fluctuating velocity components are obtained by direct substitution of mean and fluctuating components into equation (6.36) and subtraction of the mean, which leads to

$$
\frac{\partial u_{i}}{\partial t}+U_{1} \frac{\partial u_{i}}{\partial x_{j}}+u_{j} \frac{d U_{i}}{d x_{j}}-v \nabla^{2} u_{i}+\frac{1}{\rho} \frac{\partial p}{\partial x_{i}}=T_{i}
$$

The $u_{2}$-component of equation $(6.39)$ is

$$
\frac{\partial u_{2}}{\partial t}+u \frac{\partial u_{2}}{\partial x_{1}}=-\frac{1}{\rho} \frac{\partial p}{\partial x_{2}}+v \nabla^{2} u_{2}+T_{2}
$$

The equation for the fluctuating pressure may then be obtained from (6.38) making use of equation $(6.40)$ so that,

$$
\frac{\partial^{2} p}{\partial x_{1}^{2}}+\frac{\partial^{2} p}{\partial x_{3}^{2}}=\left(\frac{\partial}{\partial t}+U \frac{\partial}{\partial x_{1}}\right) \frac{\partial u_{2}}{\partial x_{2}}-\frac{\partial U}{\partial x_{2}} \frac{\partial u_{2}}{\partial x_{1}}-
$$




$$
v \nabla^{2} \frac{\partial u_{2}}{\partial x_{2}}+\frac{\partial T_{1}}{\partial x_{1}}+\frac{\partial T_{3}}{\partial x_{3}}
$$

Performing a Fourier transformation on equation (6.4I) we obtain the equation for the transform of the pressure fluctuation. The transformed equation for two dimensional disturbances is

$$
\hat{p}=-\frac{i}{\alpha}\left[(U-c) \hat{u}_{2}^{\prime}-U^{\prime} \hat{u}_{2}+\frac{i}{\alpha R}\left(\hat{u}_{2}^{\prime} \cdot-\alpha^{2} \hat{u}_{2}^{\prime}\right)\right]-\frac{i}{\alpha}\left(\hat{T}_{1}\right), \quad(6.42)
$$

where $\hat{p}$ is the transform of $p$ and the components of the equation have been non-dimensionalised with respect to a reference length, velocity and density. In our analysis we have neglected the transformed shear stress term. The equation for $\hat{p}$ in terms of the fluctuating stream function $\hat{\psi}$ is then

$$
\hat{p}=-\left[(U-c) \hat{\psi}^{\prime}-U^{\prime} \hat{\psi}+\frac{\dot{i}}{\alpha R}\left(\hat{\psi}^{\prime}+\alpha^{2} \hat{\psi}^{\prime}\right)\right]
$$

The magnitude distribution of the fluctuating pressure obtained from equation (6.43) is shown in Figure 40. The measurements of Fuchs (1969) for the mixing region of a jet exhausting into still air show a pronounced peak at the centre of the layer i.e. $y / \theta$ $=0$. The behaviour of our linearised prediction may be explained by considering the equation (6.43)。 The fluctuating pressure clearly follows the product of the $u_{1}$-fluctuation and the mean velocity towards the outer edges of the mixing. At the centre of the layer where $U$ approaches the real part of $c$, the product of the derivative of mean velocity and the $\hat{u}_{2}$-fluctuation, which is of opposite sign to the first term in the right hand side of (6.43), reduces the magnitude of the pressure fluctuation. It would appear then that in this region we are unable to 
justify our neglect of the non-linear terms. We may say that as the phase velocity of the fluctuations approaches the local mean velocity the pressure fluctuations will be produced by quadratic velocity terms; either squares of velocity fluctuations or shear stress terms. For the case of isotropic turbulence, Batchelor (1951) derived the result that,

$$
\tilde{\tilde{p}}_{\text {ISOTROPIC }}=(0.58 \rightarrow 1.0) \bar{\rho} \mathrm{v}^{2}
$$

where $\tilde{v}^{2}=u_{1}^{2}=u_{2}^{2}=u_{3}^{2}$

The products of pressure and velocity fluctuations averaged over wave length are shown in Figure 41. The products $\mathrm{pu}_{1}$ and $\overline{\mathrm{pu}}_{2}$ are of opposite sign over the whole mixing layer. The magnitude of the $\overline{p u}_{1}$ distribution is approximately twice that of the $\overline{\mathrm{pu}_{2}}$ distribution except at the positions corresponding to the sharp minimum in the $\hat{u}_{1}$ fluctuation distribution. The derivative of the $\overline{\mathrm{pu}}_{2}$ product represents the lateral pressure diffusion in the turbulent energy balance equation. Applying the normal boundary layer assumptions we may write equation $(2.32)$ as, $\left.\left.\frac{\mathrm{U}}{2} \frac{\partial}{\partial \mathrm{x}_{1}} \overline{\left(u_{1}^{2}\right.}+\overline{u_{2}^{2}}\right)+\frac{1}{2} \frac{\partial}{\partial \mathrm{x}_{2}} \overline{\left(u_{1}^{2} u_{2}\right.}+\bar{u}_{2}^{3}\right)+\frac{1}{\rho} \frac{\partial}{\partial x_{2}} \overline{p u_{2}}-$ $v\left(\overline{u_{1} \nabla^{2} u_{1}}+\overline{u_{2} \nabla^{2} u_{2}}\right)+\overline{u_{1} u_{2}} \frac{\partial U}{\partial x_{2}}=0$

The first term in equation $(6.44)$ represents the convection of turbulent energy by the mean flow. The second term represents the diffusion of turbulent energy. The third term denotes the pressure diffusion, the fourth is the viscous dissipation of turbulent energy and the fifth represents the production of 
turbulent energy. The production terms distribution in the mixing layer may be seen from the distribution of the shear term $\overline{u_{1} u_{2}}$ to be negative across the layer and have maximum magnitude at the position of maximum mean voxticity. The distribution across the layer of the pressure diffusion term is shown in Figure 42. Also shown on the figure are the experimental results of Yule (1970). It may be seen that agreement is good over most of the mixing layer except on the lower velocity side of the layer where the predicted pressure diffusion attains a small positive value and the experimental results inaicate that the term remains negative. The experimental results have been plotted such that the maximum positive value of $\frac{\partial \hat{p u}_{2}}{\partial \mathrm{x}_{2}}$ corresponds to the predicted value. In these two cases ${ }^{2}$ of production and pressure diffusion in the energy balance, where comparison may be made, the predictions agree fairly well with the measured values. However, as the computed eigenfunctions may be multiplied by an arbitrary constant and still meet the boundary conditions of the problem, only comparison of distributions of fluctuating quantities may be made.

\subsection{Summary of Results of Linear Analysis}

We will now summarise the results of the linear analysis of the turbulent mixing layer. The linear analysis provides a useful guide to the large scale structure of the shear flow. We have seen that the convection and phase velocities of the fluctuations agree with available experimental results. The 
fluctuating components of velocity and pressure have been predicted by considering the most amplifying mode in the spatial case which is considered to have a dominant effect on the local structure of flow. The maximum amplifying mode occurs at a non-dimensional wavenumber, with respect to the shear layer thickness, of 0.6 . The distribution of $\left|\hat{u}_{1}\right|^{2}$ across the mixing layex agrees with experimental results except at the edges of the layer where the theoretical prediction exhibits two minor peaks. The distribution of $\left|\vec{u}_{2}\right|^{2}$ is small by a factor of two compared with measured values. This is due to the value of wavenumber of the maximum amplifying mode, as the magnitude of the $u_{2}$-fluctuation is given by,

$$
\left|\vec{u}_{2}\right|^{2}=|i \alpha \hat{\psi}|^{2}
$$

It may be argued that the low value of the transverse velocity fluctuation may be raised by integrating the fluctuations for all wavenumbers. It has been seen that the pressure fluctuations predicted by Iinearised theory do not correspond to measured values. The major discrepancy occurs in the centre of the mixing layer where the phase velocity approaches the mean velocity. We have seen that in this region the non-linear terms may not be reasonably neglected and that the pressure fluctuations will contain contributions from the non-linear velocity product terms. We may also see this in another way: the non-linear terms will try to force a sort of isotropy on the fluctuations so that the magnitudes of the components of 
velocity become equal. In this way the non-linearity acting at the critical centre of the mixing layer will remove the dips in the $u_{2}$-fluctuation and the pressure distribution.

The discrepancies in results between the linearised theory and experiment may serve to highlight those terms where nonlinear motions dominate. We have seen that for a correct distribution of $\hat{u}_{1}$-fluctuation we may predict the Reynolds stress distribution. However, the $\vec{u}_{2}$-fluctuation and the pressure fluctuation would appear to require a non-linear solution. The successful prediction of the $\hat{\mathrm{pu}} \mathrm{u}_{2}$ correlation is almost certainly due to the coincidence of discrepancies in both cases.

On the basis of the results of the linear analysis it is clear that a useful description of the large scale motion in a turbulent shear flow may be obtained. However, the analyses so far presented have dealt with the growth and amplification of turbulent fluctuations and we have not considered, in detail, how these fluctuations are amplitude limited. We have previously argued that the small scale turbulence will contribute to an eddy viscosity. This will produce a reduction in the effective Reynolds number which will have a stabilising effect on the disturbances. We may also note that as a disturbance grows its wavenumber decreases, which will again have a stabilising effect on the fluctuation. In the next chapter we will consider a more complete analysis of the non-linear problem. We will show how the shape and rate of growth of disturbances affects the mean flow as the flow moves from a laminar to a turbulent region. 


\section{CHAPTER 7}

Finite Amplitude Effects on the Development of the Mixirg Layer

\subsection{Introduction}

In the previous chapters we have seen how linearised thecry may be used in connection with the stability of a mixing layer and the description of turbuient structure of the mixing layer. However, we have seen that the rate of growth of the linear disturbances in either space or time is very rapid and it is clear that the neglect of non-linear terms, allowed by the assumption of infinitessimal disturbances, will not be valid when the disturbances have reached finite amplitude. The added complexity of theoretical models including non-linearity is such that much work has been based on experimental observation. The separated shear layer was studied by Sato (1956, 1959). He observed that after separation the flow remained laminar for some distance until velocity fluctuations of a regular sinusoidal wave form appeared. The magnitude of the fluctuations increased until turbulence developed further downstream. The extent of the areas including sinusoidal fluctuations is sketched below, (from Sato (1959)). Inside the shaded area the wave-form is

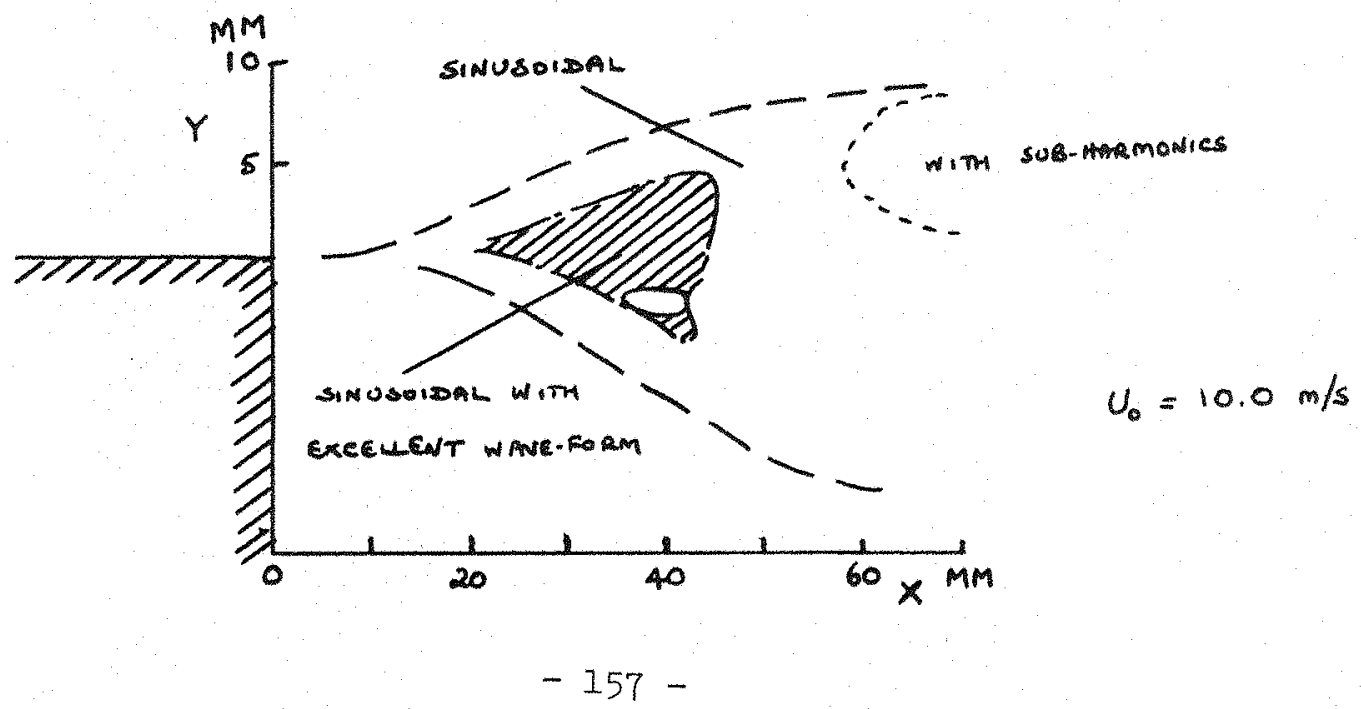


sinusoidal. Within the small region enclosed by a full-line, the amplitude of the fluctuation is very small and the waveform is not sinusoidal. Further downstream the fundamental component is accompanied by a subharmonic component.

When the amplitude of the disturbances has reached a finite value we clearly have to reform the problem of stability to take account of non-linear terms. Let us consider the vorticity equation (2.9) which we write,

$$
\frac{\partial \omega_{i}}{\partial t}+\frac{\partial \omega_{i} u_{j}}{\partial x_{j}}=\frac{\partial u_{i} \omega_{j}}{\partial x_{j}}+\nu \nabla^{2} \omega_{i}
$$

where $\omega_{i}$ and $u_{i}$ are the components of vorticity and velocity respectively. The second and third terms in equation (7.1) represent the convection and stretching of vorticity respectively. For two dimensional flow there is no vortex stretching, however it plays an important part in three-dimensional flow. Stuart (1965) noted that, for a boundary layer, if the vortex stretching terms were omitted no peaking occurred in the vorticity. Thus vortex stretching which creates a greater vorticity locally than that present at any point in the flow plays an important role in flow development.

Let us consider an example of a typical non-linear term, $v \frac{\partial u}{\partial y}$. It is clear that the linearised theory may break down for small amplitudes if the derivative becomes large. Betchov and Criminale (1967) note that if viscosity is allowed to vanish while $v$ retains some small amplitude the derivative 
$\partial u / \partial y$ becomes very large at the critical layer. At this point we may expect the non-linear terms to control the solution in place of viscous terms. The comments on the development of the boundary layer by Stuart (1965) make it clear that threedimensional effects occur before non-linearity becomes significant, however we may usefully consider the two-dimensional treatment of the non-linear problem.

The study of non-linear stability has been usefully advanced by the work of Meksyn and Stuart (1951) and Stuart (1958, 1960). The problem considered by Stuart was that of plane Poiseuille flow. He defines a stream function for the disturbed flow in the form,

$$
\begin{aligned}
& \left.\psi=\phi_{0}(z, t)+\phi_{I}(z, t) \exp \left[i \alpha\left(x-c_{r} t\right)\right]+\phi_{I} *(z, t)\right) \\
& \exp \left[-i \alpha\left(x-c_{r} t\right)\right]+\phi_{2}(z, t) \exp \left[2 i \alpha\left(x-c_{r} t\right)\right]+ \\
& \phi_{2}^{*}(z, t) \exp \left[-2 i \alpha\left(x-c_{r} t\right)\right] \\
& +-\cdots
\end{aligned}
$$

where the starred functions denote complex conjugates. The vorticity equation (7.1) may be written for two dimensional motion in terms of the stream function as,

$$
\frac{\partial \omega_{3}}{\partial t}+\frac{\partial \psi}{\partial z} \frac{\partial \omega_{3}}{\partial x}-\frac{\partial \psi}{\partial x} \frac{\partial \omega_{3}}{\partial z}=\frac{1}{R} \nabla^{2} \omega_{3}
$$

where $\quad \omega_{3}=-\nabla^{2} \psi$.

Let us define the operator $L(\alpha)$, Betchov and Criminale (1967), as 


$$
\begin{aligned}
L(\alpha)= & \left(U-c_{r}-\frac{i}{\alpha} \frac{\partial}{\partial t}\right)\left(\frac{\partial^{2}}{\partial z^{2}}-\alpha^{2}\right)-U \cdots+\frac{i}{\alpha R} . \\
& \left(\frac{\partial^{4}}{\partial z^{4}}-2 \alpha^{2} \frac{\partial^{2}}{\partial z^{2}}+\alpha^{4}\right)
\end{aligned}
$$

Then with the aid of equation (7.4) substitution of equation $(7.2)$ into $(7.3)$ and separation of harmonic components leads to the set of equations,

$$
\begin{aligned}
& L(\alpha) \phi_{1}=\phi_{2}^{\prime}\left(\phi_{1} * \cdots-\alpha^{2} \phi_{1}^{*}\right)+2 \phi_{2}\left(\phi_{1} * \cdots-\alpha^{2} \phi_{1}^{* \prime}\right)- \\
& 2 \phi_{1}^{* \prime}\left(\phi_{2}^{\prime \prime}-4 \alpha^{2} \phi_{2}^{\prime}\right)-\phi_{1}^{*}\left(\phi_{2}^{\prime \prime \prime}-4 \alpha^{2} \phi_{2}^{\prime}\right) \\
& +O\left(\phi_{2}^{*} \phi_{3}\right) \\
& L(2 \alpha) \phi_{2}=-\frac{1}{2}\left(\phi_{1}^{\prime} \phi_{1}{ }^{\prime}-\phi_{1} \phi_{1}{ }^{\prime \prime}\right)+O\left(\phi_{1}{ }^{*} \phi_{3}\right), \\
& \frac{\partial U}{\partial t \partial z}+i \alpha \frac{\partial^{2}}{\partial z^{2}}\left\{\left(\phi_{1}^{\prime} \phi_{1}^{*}-\phi_{1}^{* \prime} \phi_{1}\right)+2\left(\phi_{2}{ }^{\prime} \phi_{2}^{*}-\phi_{2}^{* \prime} \phi_{2}\right)+\right. \\
& \ldots\}=\frac{1}{R} \frac{\partial^{3} U}{\partial z^{3}}
\end{aligned}
$$

Equation $(7.7)$ may be integrated immediately with respect to z such that,

$$
\begin{aligned}
\frac{\partial U}{\partial t}+i \alpha & \frac{\partial}{\partial z}\left\{\left(\phi_{1}{ }^{\prime} \phi_{1}^{*}-\phi_{1}^{* \prime} \phi_{1}\right)+2\left(\phi_{2}{ }^{\prime} \phi_{2}{ }^{*}-\phi_{2}^{* \prime} \phi_{2}\right)+\ldots\right\} \\
& =\frac{2}{R}+\frac{1}{R} \frac{\partial^{2} U}{\partial z^{2}},
\end{aligned}
$$

where the constant of integration is given the value $(2 / R)$, the pressure gradient in laminar flow. If the $\partial / \partial t$ term in $(7.8)$ may be neglected then this equation may be viewed as a distortion in the mean profile. Stuart notes that this distortion modifies 
the transfer of energy from the mean flow to the disturbance. He applied these jdeas to the problem of Poiseuille flow. Shama (1968) used the distortion of the mean profile in his study of the turbulent boundary. He found that the modified profile introduced stronger rates of growth in the disturbance. It seems reasonable to attribute this increase in rate of growth to the appearance of inflection points in the modified profile. Stuart looked for a solution to equations $(7.5)$ and $(7.6)$, with the series terminated after the $\phi_{2}$ term, in the form

$$
\begin{aligned}
& \phi_{1}=A(t) \psi_{1}(z)+B(t) \psi_{11}(z) \\
& \phi_{2}=A^{2}(t) \psi_{2}(z)
\end{aligned}
$$

In order to obtain a solution where variations in time and $z$ are separated Stuart writes

$$
B(t)=A^{2}(t) A^{*}(t)
$$

which leads to the equation for $A(t)$ in the form,

$$
\frac{d\left(A A^{*}\right)}{d t}=2 \alpha c_{i}\left(A A^{*}\right)-2 \alpha K^{\prime}\left(A A^{*}\right)^{2}
$$

where $k$ is a complex number which may be determined from the shape of the functions $\psi_{i}(z)$ with the aid of the adjoint operator of $(7.4)$.

The solution to equation $(7.10)$ is,

$$
A A^{*}=\frac{c_{i} C \exp \left(2 \alpha c_{i} t\right)}{I+k_{i} C \exp \left(2 \alpha c_{i} t\right)},
$$


where $k_{i}$ is the imaginary part of $k$ and $C$ is an arbitrary, real constant. If $c_{i}>0$ the amplitude will increase to a maximum value of $c_{i} / k_{i}$, assuming that $k_{i}$ is notpositive. For $c_{i}<0$ and $k_{i}<0$ the disturbance takes a value $\left|c_{i}\right| /\left|k_{i}\right|$ as $t \rightarrow-\infty$ If $|A|^{2}>c_{i} / k_{i}$ then the amplitude of the disturbance grows to infinity. Stuart also considered the integral energy equation to obtain an equation of the form of equation (7.10) for the disturbance amplitude but we will consider this later as our subsequent method for the growth with space of the amplitude will be along similar lines.

A class of non-linear problems was considered by Eckhaus (1965) which included the problem of flow stability. He wrote the solution of the non-linear problem as a series in terms of eigenfunctions of the linear problem, i。e.

$$
\psi(y, t)=\sum_{n=1}^{\infty} A_{n}(t) \psi_{n}(y) 。
$$

This may also be written,

$$
\psi(y, t)=\sum_{n=1}^{\infty} B_{n} \psi_{n}(y) e^{\omega_{n} t},
$$

where $\omega_{n}$ are the eigenfrequencies and $\psi_{n}(y)$ are the orthonormal eigenfunctions. Making use of the adjoint eigenfunctions $\psi_{n}(y)$ we may write the orthogonality relationship in the form,

$$
\int_{0}^{1}\left[\frac{d \tilde{\psi}_{n}}{d y} \frac{d \psi_{m}}{d y}+k^{2} \psi_{n} \psi_{m}\right] d y=\begin{aligned}
& \text { ( } 1 \text { if } n=m \\
& \text { (0 if } n \neq m
\end{aligned}
$$


Application of the eigenfunction expansion (7.12) leads to the result,

$$
\frac{d A_{n}}{d t}-\omega_{n} A_{n}=K_{n}(t)
$$

As a test of his method Eckhaus considered the model of turbulence proposed by Burgers (1948). In this case the stream function is given by the solution to the equation,

$$
\frac{1}{R} \frac{\partial^{2} \psi}{\partial y^{2}}+\psi-\frac{\partial \psi}{\partial t}=\frac{\partial \psi^{2}}{\partial y}+R\left[\int_{0}^{1} \psi^{2} d y\right] \psi
$$

the boundary conditions on $\psi(y, t)$ being,

$$
\psi(0)=\psi(1)=0
$$

The linearised solutions are written as,

$$
\psi=\sum_{n=0}^{\infty} b_{n} e^{\omega_{n} t} \psi_{n}(y)
$$

and the $\psi_{n}(y)$ are given by

$$
\psi_{n}=\sin (n+1) \pi y
$$

with eigenfrequencies

$$
\omega_{n}=\left[1-\frac{\pi^{2}}{R}(n+1)^{2}\right]
$$

This problem is then seen to be analogous to stability theory as there exists a value of $R=R_{C R I T}=\pi^{2}$, where $R$ is a constant analogous to the Reynolds number. For $R<R_{C R I T}$ there is 
stability while for $R>R_{C R I T}$ there is instability. To consider the non-linear problem he introduces the expansion (7.12) and after some calculation he finds

$$
\begin{aligned}
\frac{d A_{n}}{d t}-\omega_{n} A_{n}= & -\frac{1}{2} R\left[\sum_{k=0}^{\infty} A_{k}^{2}\right] A_{n}+(n+1) \pi\left[-\frac{1}{2} \sum_{k=0}^{n-1} A_{k} A_{n-k-1}-\right. \\
& \left.\sum_{k=0}^{\infty} A_{k} A_{n+k+1}\right]
\end{aligned}
$$

His calculations for the first few amplitude functions, $A_{n}$, agree with those of Burgers (1948). Equation (7.18) is of the form of equation ( 7.15$)$ which in simple cases leads to the results of Stuart (1960) or Landau and Lifshitz (1953).

Finally, in this short review of non-linear methods we will consider the technique of the energy method. The method was first considered by Orr (1907), Let us define the kinetic energy per unit mass of the perturbation as,

$$
e=\frac{1}{2} u_{i} u_{i}
$$

where $u_{i}$ are the perturbations of the mean flow, $U_{i}$. We may write the rate of change of energy of the perturbation as,

$$
\begin{aligned}
\frac{\partial e}{\partial t}= & -u_{i} u_{j} \frac{\partial U_{i}}{\partial x_{j}}-\frac{1}{R} \frac{\partial u_{i}}{\partial x_{j}} \frac{\partial u_{i}}{\partial x_{j}}+\frac{\partial}{\partial x_{j}}\left[\frac{u_{i} u_{i}}{2}\left(u_{j}+u_{j}\right)+\frac{I}{2 R} \frac{\partial}{\partial x_{j}} u_{i} u_{i}-\right. \\
& p u_{j} I
\end{aligned}
$$

where the components in the equation are suitably non-dimensionalised. If we consider the integral of (7.20) over a volume bounded by walls or the nodes of a periodic velocity field the term in brackets will not contribute and we may write, 


$$
\frac{\partial E}{\partial t}=\frac{\partial}{\partial t} \iint_{V} \int e d V=-\iint_{V} \int u_{i} u_{j} \frac{\partial U_{i}}{\partial x_{j}} d V-\frac{j}{R} \iiint_{V} \frac{\partial u_{i}}{\partial x_{j}} \frac{\partial u_{i}}{\partial x_{j}} d V
$$

If the total energy is stationary then $\frac{\partial E}{\partial t}=0$ and the production term will equal the dissipation. We then have,

$$
R=\frac{\iint_{V} \int \frac{\partial u_{i}}{\partial x_{j}} \frac{\partial u_{i}}{\partial x_{j}} d V}{-\iiint_{V} u_{i} u_{j} \frac{\partial u_{i}}{\partial x_{j}} d V}
$$

Use may be made of the calculus of variations to find the extreme value of $R$, that is the critical Reynolds number.

Stuart (1958) used the energy method for two dimensional disturbances in Poiseuille flow. The integral energy equation may then be written,

$$
\begin{aligned}
& \frac{\partial}{\partial t} \iint \frac{1}{2}\left(u_{1}^{2}+u_{3}^{2}\right) d x_{1} d x_{3}=\iint\left(-\overline{u_{1} u_{3}}\right) \frac{\partial U}{\partial z} d x_{1} d x_{3}- \\
& \quad \frac{1}{R} \iint\left(\frac{\partial u_{3}}{\partial x_{1}}-\frac{\partial u_{1}}{\partial x_{3}}\right)^{2} d x_{1} d x_{3}
\end{aligned}
$$

The equation for the mean flow is

$$
\frac{\partial U}{\partial t}+\frac{\partial}{\partial x_{3}}\left(\overline{u_{1} u_{3}}\right)=\frac{2}{R}+\frac{1}{R} \frac{\partial^{2} U}{\partial x_{3}^{2}}
$$

If we assume that $\partial U / \partial t$ may be neglected then equation $(7.24)$ may be integrated for $U\left(x_{3}\right)$ and with equation $(7.23)$ we obtain, 


$$
\gamma_{1} \frac{\partial a^{2}}{\partial t}=\gamma_{2} a^{4}-\gamma_{3} \alpha^{2} \operatorname{Ra}^{4}-\frac{\gamma_{4} a^{2}}{R},
$$

where $a$ is the amplitude of the function and,

$$
\begin{gathered}
r_{I}=\int_{0}^{1}\left\{\left|\phi^{\prime}\right|^{2}+\alpha^{2}|\phi|^{2}\right\} d x_{3}, \gamma_{2}=4 \int_{0}^{1} x_{3}\left(\phi_{i} \phi_{r}^{\prime}-\phi_{r} \phi_{i}^{\prime}\right) d x_{3}, \\
r_{3}=4 \int_{0}^{1}\left(\phi_{i} \phi_{r}^{\prime}-\phi_{\left.r_{i} \phi^{\prime}\right) d x_{3}, r_{4}}=2 \int_{0}^{1}\left\{\left|\phi^{\prime \prime}\right|^{2}+2 \alpha^{2}\left|\phi^{\prime}\right|^{2}+\right.\right. \\
\left.\alpha^{4}|\phi|^{2}\right\} d x_{3},
\end{gathered}
$$

the suffixes $r$ and $i$ denote real and imaginary parts and $\phi$ is defined as in equation (7.2). We may write equation (7.25) as,

$$
\frac{d a^{2}}{d t}=\beta_{1} a^{2}-\beta_{2} a^{2}-\beta_{3} a^{4}
$$

The solution to equation $(7.26)$ is in the form,

$$
a^{2}=\frac{c e^{\left(\beta_{1}-\beta_{2}\right) t}}{1+\left(\frac{\beta_{3}}{\beta_{1}-\beta_{2}}\right) c e^{\left(\beta_{1}-\beta_{2}\right) t}}
$$

Now $\beta_{1}-\beta_{2}=2 \alpha c_{i}$, from linear stability theory. Thus we see that equation $(7.27)$ is equivalent to equation $(7.11)$. The equilibrium amplitude of the disturbance occurs when $\frac{\mathrm{da}^{2}}{\mathrm{dt}}=0$ and may be shown to be,

$$
a_{e}^{2}=\frac{\beta_{I}-B_{2}}{\beta_{3}}=\frac{\gamma_{2}\left(R-R_{C R I T}\right)}{\gamma_{3} \alpha R^{2}}
$$

Thus it may be seen that the equilibrium amplitude is proportional 
to the square root of the difference between the flow Reynolds number and the critical Reynolds number. Stuart showed that the critical Reynolds number agreed reasonably with the calculations of Thomas (1953) although the correct eigensolutions were not available for integration.

We will now present our formulation of the non-linear problem. The integral equation of mean and fluctuating components of the flow will be written as ordinary differential equations in $x_{1}$ in terms of a number of shape parameters of the mean flow and the amplitude of the disturbances. The distributions for the fluctuations will be calculated using a modified solution of the stability problem. The technique to be used is similar to that successfully applied to the wake by Ru-Sue Ko et. al. (1970).

\subsection{Governing Differential and Integral Equations}

In Chapter 2 we obtained the integral equations of the mean flow by integrating across the layer the mean momentum and energy equations and the turbulent energy equation. These were then written as ordinary differential equations in the independent variable $x$ by considering the differentiation of integrals. The final equations in Chapter 2 were derived with the assumption that the fluctuations were zero at the limits of the integration. In this chapter we will not use that assumption as it will be seen that the disturbance distributions used in our formulation decrease exponentially outside the layer.

Referring to Chapter 2 we may write the integral equations of the mean flow and fluctuations as (i) the mean momentum equation, 


$$
\int_{-\delta_{2}}^{\delta_{I}} \frac{\partial}{\partial x}\left[\left(U-U_{1}\right)\left(U-U_{2}\right)+\left(\vec{u}^{2}-\vec{v}^{2}\right)\right] d y+\left[v_{2} U_{1}-U_{2} V_{I}\right]=0,
$$

(ii) the mechanical energy equation,

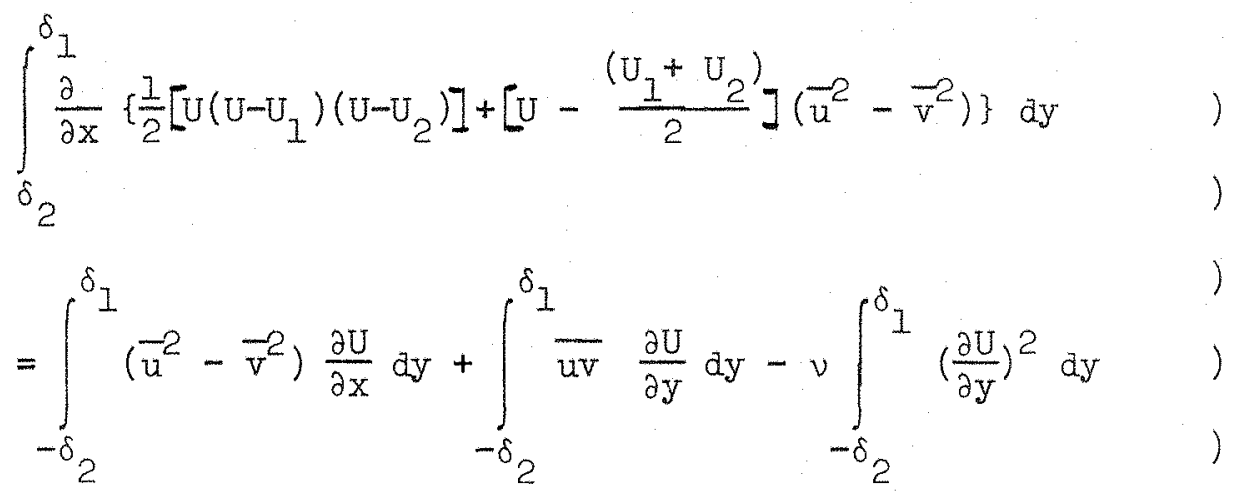

and (iii) the mean fluctuation energy equation,

$$
\begin{aligned}
& \frac{1}{2} \int_{-\delta_{2}}^{\delta_{I}} \frac{\partial}{\partial x}\left[u\left(\bar{u}^{2}+\bar{v}^{2}\right)+\bar{u}^{3}+\overline{u v}^{2}+2 \overline{u p} \frac{\bar{p}}{p}\right] d y=-\int_{-\delta_{2}}^{\delta_{1}}\left(\bar{u}^{2}-\vec{v}^{2}\right) \frac{\partial U}{\partial x} d y- \\
& \int_{-\delta_{2}}^{\delta_{1}} \overline{u v} \frac{\partial U}{\partial y}-v \int_{-\delta_{2}}^{\delta_{I}}\left[\left(\frac{\overline{\partial u}}{\partial x}\right)^{2}+\left(\overline{\frac{\partial u}{\partial y}}\right)^{2}+\left(\frac{\partial \bar{v}}{\partial x}\right)^{2}+\left(\frac{\overline{\partial v}}{\partial y}\right)^{2}\right] d y
\end{aligned}
$$

Making use of equation (2.24) we may write equation (7.29)

$$
\begin{aligned}
& \frac{\mathrm{d}}{\mathrm{dx}} \int_{-\delta_{2}}^{\delta_{1}}\left[\left(\mathrm{U}-\mathrm{U}_{1}\right)\left(\mathrm{U}-\mathrm{U}_{2}\right)+\left(\overrightarrow{\mathrm{u}}^{2}-\overrightarrow{\mathrm{v}}^{2}\right)\right] \mathrm{dy}-\frac{\mathrm{d} \delta_{2}}{\mathrm{dx}}\left(\overline{\mathrm{u}}_{2}{ }^{2}-\overline{\mathrm{v}}_{2}{ }^{2}\right)-\text { ) } \\
& \frac{d \delta_{1}}{d x}\left(\bar{u}_{1}^{2}-\bar{v}_{1}^{2}\right)+\left[v_{2} U_{1}-U_{2} V_{1}\right]=0
\end{aligned}
$$

where the subscripts 1 and 2 on the fluctuations refer to their values at the upper and lower limits of integration respectively. 
Equation (7.30) becomes,

$$
\begin{aligned}
& \left.\frac{d}{d x} \int_{-\delta_{2}}^{\delta_{1}}\left\{\frac{1}{2}\left[u\left(U-U_{1}\right)\left(U-U_{2}\right)\right]+\left[u-\frac{\left(U_{1}+U_{2}\right)}{2}\right]\left(\vec{u}^{2}-\vec{v}^{2}\right)\right\} d y\right) \\
& +U_{d}\left\{\frac{d \delta_{2}}{d x}\left(\bar{u}_{2}^{2}-\bar{v}_{2}^{2}\right)-\frac{d \delta_{1}}{d x}\left(\bar{u}_{1}^{2}-\bar{v}_{1}^{2}\right)\right\} \\
& \left.=\int_{-\delta_{2}}^{\delta_{1}}\left(\vec{u}^{2}-\vec{v}^{2}\right) \frac{\partial U}{\partial x} d y+\int_{-\delta_{2}}^{\delta_{I}} \overline{u v} \frac{\partial U}{\partial y} d y-v \int_{-\delta_{2}}^{\delta_{1}}\left(\frac{\partial U}{\partial y}\right)^{2} d y, \quad\right)
\end{aligned}
$$

where $U_{d}=\frac{\left(U_{1}-U_{2}\right)}{2}$

Equation $(7.30)$ becomes,

$$
\begin{aligned}
& \left.\frac{1}{2} \frac{d}{d x} \int_{-\delta_{2}}^{\delta_{1}}\left[u\left(\vec{u}^{2}+\bar{v}^{2}\right)+2 \frac{\overline{u p}}{p}\right] d y+\frac{1}{2} \frac{d}{d x} \int_{-\delta_{2}}^{\delta_{1}}\left(\bar{u}^{3}+\overline{u v}^{2}\right) d y\right) \\
& -\frac{d \delta_{2}}{d x}\left[u_{2} \frac{\left(\bar{u}_{2}^{2}+\bar{v}_{2}^{2}\right)}{2}+\frac{\overline{u_{2} p_{2}}}{\rho}\right]-\frac{d \delta_{1}}{d x}\left[u_{1} \frac{\left(\bar{u}_{1}^{2}+\bar{v}_{1}^{2}\right)}{2}\right) \\
& \left.+\frac{\overline{u_{1} p_{1}}}{\rho}\right]-\frac{1}{2}\left\{\frac{d \delta_{2}}{d x}\left(\bar{u}_{2}^{3}+{\overline{u_{2} v_{2}^{2}}}^{2}+\frac{d \delta_{1}}{d x}\left(\bar{u}_{1}^{3}+{\overline{u_{1} v_{1}}}^{2}\right)\right\},\right) \\
& =-\int_{-\delta_{2}}^{\delta_{1}}\left(\vec{u}^{2}-\vec{v}^{2}\right) \frac{\partial U}{\partial x} d y-\int_{-\delta_{2}}^{\delta_{1}} \frac{\partial U}{u v} \frac{\partial U}{\partial y} d y-v \int_{-\delta_{2}}^{\delta_{1}}\left[\left(\frac{\partial u}{\partial x}\right)^{2}+\right) \\
& \left(\frac{\partial u}{\partial y}\right)^{2}+\left(\frac{\overline{\partial v}_{\partial x}}{2}+\left(\frac{\overline{\partial v}^{2}}{\partial y}\right)^{2}\right] d y
\end{aligned}
$$


We will now non-dimensionalise equations $(7.32),(7.33)$ and (7.34) with respect to a reference length $l$ and the velocity difference $\left(U_{1}-U_{2}\right) / 2$. The pressure is non-dimensionalised with respect to $\rho\left(U_{1}-U_{2}\right)^{2} / 4$. Equations $(7.32),(7.33)$ and $(7.34)$ may then be written,

$$
\begin{aligned}
& \left.\frac{d}{d x^{*}} \int_{\delta_{2}^{*}}^{\delta_{1}^{*}}\left[\left(u^{*}-U_{1}^{*}\right)\left(u^{*}-U_{2}^{*}\right)+\left(\bar{u}^{2}-\bar{v}^{2}\right)\right] d y^{*}-\frac{d}{d x^{*}} \cdot\right) \\
& \left.\left(\bar{u}_{2}^{* 2}-\bar{v}_{2}^{* 2}\right)-\frac{d \delta_{1}}{d x^{*}}\left(\bar{u}_{1}{ }^{2}-\bar{v}_{1}^{*}\right)+\left[v_{2}{ }^{2} U_{1}^{*}-U_{2}^{*} v_{1}^{*}\right]=0\right)
\end{aligned}
$$

$\frac{d}{d x^{*}} \int_{-\delta_{2}^{*}}^{\delta_{1}^{*}}\left\{\frac{1}{2}\left[U^{*}\left(U^{*}-U_{1}^{*}\right)\left(U^{*}-U_{2}^{*}\right)\right]+\left[U^{*}-\frac{\left(U_{1}^{*}+U_{2}^{*}\right)}{2}\right]\left(\bar{u}^{2}-\bar{v}^{*^{2}}\right)\right\} d y^{*}$$$
+\left\{\frac{d \delta_{2}^{*}}{d x^{*}}\left(\bar{u}_{2}{ }^{2}-\bar{v}_{2}{ }^{2}\right)-\frac{d \delta_{1}^{*}}{d x^{*}}\left(\bar{u}_{1}{ }^{2}-\bar{v}_{1}{ }^{2}\right)\right\}
$$$$
=\int_{-\delta_{2}^{*}}^{\delta_{1}^{*}}\left(\bar{u}^{*}-\bar{v}^{*}\right) \frac{\partial U^{*}}{\partial x^{*}} d y^{*}+\int_{-\delta_{2}^{*}}^{\delta_{1}^{*}} \overline{u^{*} v^{*}} \frac{\partial U^{*}}{\partial y^{*}} d y^{*}
$$$$
-\frac{1}{R_{l}} \int_{-\delta}^{\delta_{2}^{*}}\left(\frac{\partial U^{*}}{\partial y^{*}}\right)^{2} d y^{*}
$$

$$
\begin{aligned}
& \text { and } \frac{1}{2} \frac{d}{d x^{*}} \int_{-\delta_{2}^{*}}^{\delta_{1}^{*}}\left[U^{*}\left(\overline{u^{*}}+\overline{v^{*}}\right)+2 \overline{u^{*} p^{*}}\right] d y^{*}+\frac{1}{2} \frac{d}{d x^{*}} \int_{-\delta_{2}^{*}}^{\delta_{1}^{*}}\left(\overline{u^{*}}+\overline{u^{*} v^{* 2}}\right) d y \\
& -\frac{d \delta_{2}^{*}}{d x^{*}}\left[u_{2} * \frac{\left(\bar{u}_{2}^{* 2}+\bar{v}_{2}^{*}\right)}{2}+\bar{u}_{2}^{*} p_{2}^{*}\right]-
\end{aligned}
$$




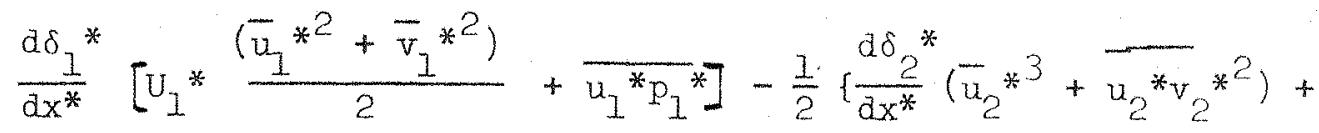

$$
\begin{aligned}
& \left.\frac{d \delta_{1}^{*}}{d x^{*}}\left(\bar{u}_{1}^{* 3}+\overline{u_{1}^{*} v_{1}{ }^{2}}\right)\right\} \\
& =-\int_{-\delta_{2}^{*}}^{\delta_{1}^{*}}\left(\bar{u}^{*}-\bar{v}^{*}\right) \frac{\partial U^{*}}{\partial x^{*}} d y^{*}-\int_{-\delta_{2}^{*}}^{\delta_{1}^{*}} \frac{}{u^{*} v^{*}} \frac{\partial U^{*}}{\partial y^{*}} d y^{*}- \\
& \frac{I}{R_{\ell}} \int_{-\delta]^{*}}^{\delta}\left[\left(\frac{\partial u^{*}}{\partial x^{*}}\right)^{2}+\left(\frac{\partial u^{*}}{\partial y^{*}}\right)^{2}+\left(\frac{\partial v^{*}}{\partial x^{*}}\right)^{2}+\left(\frac{\partial v^{*}}{\partial y^{*}}\right)^{2}\right] d y^{*},
\end{aligned}
$$

where all non-dimensional quantities are starred, and the Reynolds number $R_{\ell}$ is given by,

$$
R_{l}=\frac{\left(U_{1}-U_{2}\right)_{l}}{2 v}
$$

\subsection{Shape Assumption for the Mean Flow}

In this section we will assume that the unknowns in our equations may be expressed in terms of a number of shape parameters. These will then be determined from the integral equations. In their study of the incompressible wake Ru-Sou Ko et. al. (1970) were able to express the mean velocity profile in terms of two parameters, the wake half-width and the velocity defect. In Chapter 3 we saw that when the mean velocity profiles for both laminar and turbulent mixing layers were plotted as a function of $n$, where $n$ is given by, 


$$
n=\frac{y^{*}-b^{*}\left(x^{*}\right)}{\theta^{*}\left(x^{*}\right)}
$$

then the profiles coincided. Sato (1956), whose results on the transition of the mixing layer we will use later, noticed this in his measurements of the mean velocity profiles of a mixing layer before and after transition from laminar to turbulent flow. In equation $(7.39), b^{*}(x)$ is the value of $\mathrm{y}^{*}$ at which $\mathrm{U}=\frac{\mathrm{U}_{1}+\mathrm{U}_{2}}{2}$, and $\theta^{*}$ is the non-dimensional momentum thickness defined by,

$$
\theta^{*}=\int_{-\infty}^{\infty}\left(U_{1}^{*}-U^{*}\right)\left(U^{*}-U_{2}^{*}\right) d y^{*}
$$

We will then write the mean velocity profile as,

$$
U^{*}\left(x^{*}, y^{*}\right)=\frac{U_{1}^{*}+U_{2}^{*}}{2}+f(n),
$$

and we will further write $n$ as,

$$
n=\frac{y^{*}-b^{*}(\omega)}{\theta_{1}^{*}\left(x^{*}\right)+\theta_{2}^{*}\left(x^{*}\right)}=\frac{y^{*}-b^{*}\left(x^{*}\right)}{\theta^{*}\left(x^{*}\right)}
$$

where $\left.\theta_{2}^{*}\left(\mathrm{x}^{*}\right)=\int_{-\infty}^{0}\left(U_{1}^{*}-\mathrm{U}^{*}\right)\left(\mathrm{U}^{*}-\mathrm{U}_{2}^{*}\right) d \mathrm{y}^{*}\right)$

and $\left.\theta_{1}^{*}\left(x^{*}\right)=\int_{0}^{\infty}\left(U_{I}^{*}-U^{*}\right)\left(U^{*}-U_{2}^{*}\right) d y^{*}\right)$

In this manner we are able to define more clearly the width of the mixing layer. We will now change the independent variables $\left(x^{*}, y^{*}\right)$ to $(\xi, n)$ in equations $(7.35),(7.36)$ and 
(7.37). The transformation of variables follows the following rules,

$$
\left.\xi=x^{*} \quad ; \quad n=\frac{y^{*}-b^{*}}{\theta^{*}} ;\right)
$$

$\left.\frac{\partial}{\partial x^{*}}=\frac{\partial}{\partial \xi}-\left\{\frac{I}{\theta^{*}} \frac{d b^{*}}{d x^{*}}+\frac{\eta}{\theta^{*}} \frac{d \theta}{d x^{*}}\right\} \frac{\partial}{\partial \eta} ; \quad \frac{\partial}{\partial y^{*}}=\frac{I}{\theta^{*}} \frac{\partial}{\partial \eta}, \ldots\right)$

We will also alter the range of integration to an integration across the momentum thickness. Then equations $(7.35)$ and $(7.36)$ become,

$$
\begin{aligned}
& \left.\frac{d}{d \xi}\left\{\frac{\theta^{*}}{4}\left[\left(U_{1}^{*}-U_{2}^{*}\right)^{2}+4 \beta_{2}+4\left(I_{1}-I_{2}\right)\right]\right\}-\frac{d \theta}{d \xi}\left(\bar{u}_{2}^{*}{ }^{2}-\bar{v}_{2}^{*}{ }^{2}\right)\right) \\
& -\frac{d \theta_{1}^{*}}{d \xi}\left(\bar{u}_{1}^{* 2}-\bar{v}_{1}^{* 2}\right)+\left[V_{2}^{*} U_{1}^{*}-U_{2}^{*} v_{1}^{*}\right]=0
\end{aligned}
$$

and

$$
\begin{array}{ll}
\frac{d}{d \xi}\left\{\frac{\theta^{*}}{8}\left[8 \beta_{3}+4\left(U_{1}^{*}+U_{2}^{*}\right) \beta_{2}-2\left(U_{1}^{*}-U_{2}^{*}\right)^{2} \beta_{1}-\left(U_{1}^{*}-U_{2}^{*}\right)^{2}\right)\right. & ) \\
\left.\left.\left(U_{1}^{*}+U_{2}^{*}\right)+8\left(I_{3}-I_{4}\right)\right]\right\}+\left\{\frac{d \theta^{*}}{d \xi}\left(\bar{u}_{2}^{* 2}-\bar{v}_{2}^{*}{ }^{2}\right)-\right. & ) \\
\left.\frac{d \theta_{1} *}{d \xi}\left(\bar{u}_{1}^{*}-\bar{v}_{I}^{*}\right)\right\} & ) \\
=\frac{-d b^{*}}{d \xi} I_{5}-\frac{d \theta^{*}}{d \xi} I_{6}+I_{7}-\frac{1}{R_{\theta}} \beta_{4}, & )
\end{array}
$$

where,

$$
\beta_{1}=\int_{-n_{2}}^{n_{1}} f(n) d n \quad ; \quad \beta_{2}=\int_{-n_{2}}^{n_{1}} f^{2}(n) d n
$$




$$
\begin{aligned}
& \beta_{3}=\int_{-n_{2}}^{n_{1}} f^{3}(n) d n \quad ; \quad \beta_{4}=\int_{-n_{2}}^{n_{1}}\left(\frac{d f}{d n}\right)^{2} d n \\
& I_{1}=\int_{-n_{2}}^{n_{1}} \frac{u^{*}}{2} d n \quad I_{2}=\int_{-n_{2}}^{n_{1}} \overline{v^{*}} d n \\
& I_{3}=\int_{-n_{2}}^{n_{1}} f \bar{u}^{2} d n \quad ; \quad I_{4}=\int_{-n_{2}}^{n_{1}} f \bar{v}^{2} d n \\
& I_{5}=\int_{-n_{2}}^{n_{1}}\left(\bar{u}^{*^{2}}-\bar{v}^{*^{2}}\right) \frac{d f}{d n} d n ; \quad I_{6}=\int_{-n_{2}}^{n_{1}}\left(\overline{u^{*}}-\overline{v^{*}}\right) n \frac{d f}{d n} d n \\
& I_{7}=\int_{-n_{2}}^{n_{1}} \overline{u^{*} v^{*}} \frac{d f}{d n} d n \\
& n_{1}=\frac{\theta_{1}^{*}-b^{*}}{\theta^{*}} \quad ; \quad n_{2}=\frac{\theta_{2}^{*}-b^{*}}{\theta^{*}} ;
\end{aligned}
$$

and

$$
R_{\theta}=\frac{\left(U_{1}-U_{2}\right) \theta}{2 v}
$$

Equation $(7.37)$ becomes,

$$
\begin{aligned}
& \frac{d}{d \xi}\left\{\theta *\left[\frac{\left(U_{1}^{*}+U_{2}^{*}\right)}{2}\left(I_{1}+I_{2}\right)+\left(I_{3}+I_{4}\right)+2 I_{8}+I_{9}\right]\right\}- \\
& \frac{d \theta_{2}^{*}}{d \xi}\left[U_{2}^{*}\left(\bar{u}_{2}^{* 2}+\bar{v}_{2}^{*^{2}}\right)+\overline{\left.2 u^{*}{ }_{2} p_{2}^{*}\right]}-\frac{d \theta_{1}^{*}}{d \xi}\left[U_{1}^{*}\left(\bar{u}^{*}+\bar{v}^{2}\right)+\right.\right.
\end{aligned}
$$




$$
\begin{aligned}
& 2 u_{1}^{*} p_{1}^{* j}-\left\{\frac{d \theta_{2}^{*}}{d \xi}\left(\bar{u}_{2}^{*}+\overline{u_{2}{ }^{*} v_{2} *^{2}}\right)+\frac{d \theta_{1}^{*}}{d \xi}\left(\bar{u}_{1} *^{3}+\overline{u_{1}{ }^{*} v_{1}{ }^{2}}\right)\right\} \\
& =2 \frac{d b^{*}}{d \xi} I_{5}+2 \frac{d \theta^{*}}{d \xi} I_{6}-2 I_{7}-\frac{2}{R_{\theta}} I_{10}, \\
& \text { where } I_{8}=\int_{-n_{2}}^{n_{1}} \overline{u^{*} p^{*}} d n \quad ; \quad I_{9}=\int_{-n_{2}}^{n_{1}}\left(\overline{u^{*}}+\overline{u^{*} v^{*}}\right) d n \text {; } \\
& \text { and } I_{10}=\int_{-n_{2}}^{n_{1}}\left\{\left(\frac{\partial \tilde{u}^{*}}{\partial \eta}\right)^{2}+\left(\overline{\frac{\partial \tilde{u}^{*}}{\partial \xi^{*}}}\right)^{2}+\left(\overline{\frac{\partial \tilde{v}^{*}}{\partial \eta}}\right)^{2}+\left(\frac{\partial v^{*}}{\partial \xi^{*}}\right)^{2}\right\} d \eta
\end{aligned}
$$

where $\xi^{*}$ is the axial co-ordinate non-dimensionalised with respect to the local thickness, i。e.

$$
\xi_{3}^{*} \theta^{*}=\xi
$$

Equations $(7.45),(7.46)$ and $(7.47)$ are differential equations of the first order in the independent variable $\xi$ 。 The unknown quantities are $\theta^{*}(\xi), b^{*}(\xi)$ and the variation of the I-integrals as functions of $\xi$. In order to generate the disturbance distributions we will now make use of the results of a linear stability analysis.

\subsection{Shape Assumptions for the Fluctuations}

Experimental investigations of the transition of a free shear layer from laminar to turbulent flow have shown that the initial development of the flow is dominated by a disturbance of a single frequency. It has been noted, (Browand (1966), 
Freymuth (1966) and Sato (1956)), that this frequency corresponds to the frequency at which maximum amplification is preaicted using spatial analysis in linear stability theory。 Thus in order to generate the disturbance terms in the integral equations of the mean flow we will use a form of linearised stability theory. We have seen in previous sections that the disturbance distributions of a single frequency component predicted from the eigensolutions of the Orr-Sommerfeld equation may be used to represent the turbulent fluctuations. The integral equations derived above are exact and the only assumptions to be made in order to solve the ordinary differential equations are that the mean flow may be represented in terms of a number of shape parameters and that the disturbance distributions may be generated from solution of the Orr-Sommerfeld equation. Thus, as in Chapter 2, we will introduce a Fourier transform of the velocity and pressure fluctuations such that, $\varepsilon(\xi) . \hat{u}\left(\alpha^{*}, \omega^{*} ; \eta\right)=\frac{1}{(2 \pi)^{2}} \int_{-\infty}^{\infty} \int_{-\infty}^{\infty} \exp \left\{-i\left(\alpha^{*} \xi^{*}-\omega^{*} t^{*}\right)\right\} u^{*}\left(\xi^{*}, t^{*} ; \eta\right) d \xi^{*} d t^{*}$
$\varepsilon(\xi) . \hat{v}\left(\alpha^{*}, \omega^{*} ; \eta\right)=\frac{1}{(2 \pi)^{2}} \int_{\infty}^{\infty} \exp \left\{-i\left(\alpha^{*} \xi^{*}-\omega^{*} t^{*}\right)\right\} v^{*}\left(\xi^{*}, t^{*} ; \eta\right) d \xi^{*} d t^{*}$ $\varepsilon(\xi) \cdot \hat{p}\left(\alpha^{*}, \omega^{*} ; \eta\right)=\frac{1}{(2 \pi)^{2}} \int_{\infty}^{\infty} \int_{\infty}^{\infty} \exp \left\{-i\left(\alpha^{*} \xi^{*}-\omega^{*} t^{*}\right) p^{*}\left(\xi^{*}, t^{*} ; \eta\right) d \xi^{*} d t^{*}\right.$ $(7.48)$

where $\alpha^{*}=\alpha \theta^{*}$, the compex wavenumber and $\omega^{*}=\omega \theta^{*}$ is the local real angular frequency. The amplitude of the disturbances is then given by, 


$$
A(\xi)=\varepsilon(\xi) \exp \left(-\alpha_{i}^{*} \xi^{*}\right)
$$

Making use of expressions (7.48) we obtain, as in Chapter 2 , an equation for the distribution of $\hat{v}(n)$. This is of similar from to the Orr-Sommerfeld equation,

$$
(f-\bar{c})\left(\frac{\partial^{2}}{\partial \eta^{2}}-\alpha^{*}\right) \hat{v}-\frac{\partial^{2} \hat{f}}{\partial \eta^{2}} \hat{v}+\frac{i}{\alpha^{* R}} \cdot\left\{\frac{\partial^{4 \hat{v}}}{\partial \eta^{4}}-2 \alpha^{2} \frac{\partial^{2} \hat{v}}{\partial \eta^{2}}+\alpha{ }^{4} \hat{v}\right\}=0(7.50)
$$

where $\bar{c}=\frac{\omega^{*}}{\alpha^{*}}-\frac{\left(U_{1}^{*}+U_{2}^{*}\right)}{2}$.

The boundary conditions on the function $\hat{v}(n)$ are,

$$
\hat{v}=\frac{\partial \hat{v}}{\partial \eta}=0 \quad \text { as } n \rightarrow \pm \infty
$$

Equation (7.50), together with the boundary conditions (7.51) constitutes an eigenvalue problem. For a given function $f(n)$ we have,

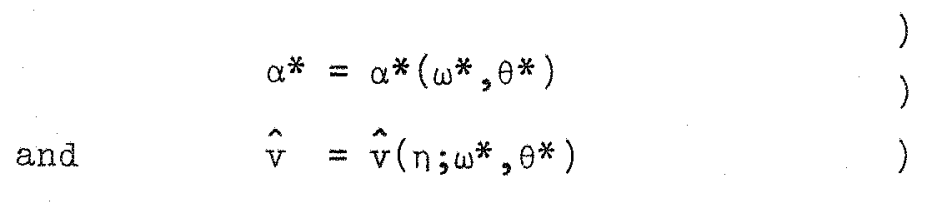

We will now write the integrals $I_{i}, i=1 \ldots 10$ as functions of the single parameter $\theta *$ and the amplitude square of the disturbances, $|A|^{2}$. Then the integrals can be written in the form,

$$
I_{i}=k_{i}\left(\theta^{*}\right)|A|^{2}, \quad i=1,2 \ldots 10
$$

when only the fundamental mode is included the terms associated with the triple correlation will vanish. 
Using the definition $(7.50)$, equations $(7.45),(7.46)$ and $(7.47)$ become three first order differential equations for the three parameters, $\theta^{*}, \mathrm{~b}^{*}$ and $|A|^{2}$. These equations may be solved as an initial value problem. In order to define the disturbance magnitude we will set

$$
k_{1}+k_{2}=1
$$

Using equation (7.53) we may write equations $(7.45),(7.46)$ and $(7.47)$ as,

$$
\begin{aligned}
& \left.\frac{1}{4}\left[-\left(U_{1}^{*}-U_{2}^{*}\right)^{2}+4 B_{2}+4|A|^{2}\left(k_{1}-k_{2}\right)+4 \theta^{*}|A|^{2}\left(\frac{d k_{1}}{d \theta^{*}}-\frac{d k_{2}}{d \theta^{*}}\right)\right]\right)
\end{aligned}
$$

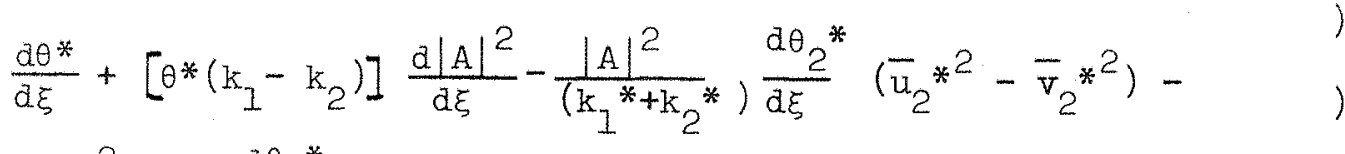

$$
\begin{aligned}
& \frac{|A|^{2}}{\left(\mathrm{k}_{1}^{*}+\mathrm{k}_{2}^{*}\right)} \frac{\mathrm{d \theta}{ }_{1}^{*}}{\mathrm{~d} \xi}\left(\bar{u}_{1}^{* 2}-\bar{v}_{1}^{*}\right)+\left[\mathrm{V}_{2}^{*} \mathrm{U}_{1}^{*}-\mathrm{U}_{2}^{*} \mathrm{~V}_{1}^{*}\right]=0 \text {, }
\end{aligned}
$$

$\frac{1}{8}\left[8 \beta_{3}+4\left(U_{1}^{*}+U_{2}^{*}\right) \beta_{2}-2\left(U_{1}^{*}-U_{2}^{*}\right)^{2} \beta_{1}-\left(U_{1}^{*}-U_{2}^{*}\right)^{2}\left(U_{1}^{*}+U_{2}^{*}\right)\right)$ $+8|A|^{2}\left\{\left(k_{3}-k_{4}+k_{6}\right)+0 *\left(\frac{d k_{3}}{d \theta^{*}}-\frac{d k_{4}}{d \theta *}\right)\right] \frac{d \theta^{*}}{d \xi}+\left[\theta *\left(k_{3}-k_{4}\right)\right]$ $\left.\frac{d|A|^{2}}{d \xi}+T|A|^{2} k_{6}\right] \frac{d b^{*}}{d \xi}+\frac{|A|^{2}}{\left(k_{1}{ }^{*}+k_{2}{ }^{*}\right)}\left\{\frac{d 2^{*}}{d \xi}\left(\bar{u}_{2}{ }^{2}-\bar{v}_{2}{ }^{2}\right)-\right.$ $\left.\frac{d \theta_{I} *}{d \xi}\left(\bar{u}_{I}{ }^{2}-\bar{v}_{1} *^{2}\right)\right\}-I|A|^{2} k_{7}-\frac{I}{R_{\theta}} B_{4} I=0$

and

$$
\left[|A|^{2}\left\{\frac{\left.U_{1}^{*}+U_{2}^{*}\right)}{2}\left(k_{1}+k_{2}\right)+\left(k_{3}+k_{4}-2 k_{6}+2 k_{8}\right)\right\}+\right.
$$




$$
\begin{aligned}
& \left.|A|^{2} \theta^{*}\left\{\frac{\left(U_{1}^{*}+U_{2}^{*}\right)}{2}\left(\frac{d k_{1}}{d \theta^{*}}+\frac{d k_{2}}{d \theta^{*}}\right)+\left(\frac{d k_{3}}{d \theta^{*}}+\frac{d k_{4}}{d \theta^{*}}+2 \frac{d k_{8}}{d \theta^{*}}\right)\right\}\right] \frac{d \theta^{*}}{d \xi} \\
& +\left[\theta *\left\{\frac{\left(U_{1}^{*}+U_{2}^{*}\right)}{2}\left(k_{1}+k_{2}\right)+\left(k_{3}+k_{4}+2 k_{8}\right)\right\}\right] \frac{d|A|^{2}}{d \xi}- \\
& {\left[2|\mathrm{~A}|^{2} \mathrm{k}_{5}\right] \frac{\mathrm{db} *}{\mathrm{~d} \xi}-\frac{|\mathrm{A}|^{2}}{\left(\mathrm{k}_{1}^{*}+\mathrm{k}_{2}^{*}\right)}\left\{\frac { \mathrm { d \theta } } { \mathrm { d } \xi } \left[\mathrm{U}_{2} *\left(\bar{u}_{2}{ }^{2}+\overline{\mathrm{v}}_{2}{ }^{2}\right)+\right.\right.} \\
& \left.\left.\overline{2 u_{2}{ }^{*} p_{2}{ }^{*}}\right]-\frac{|A|^{2}}{\left(k_{1}^{*}+k_{2} *\right.}\right)\left\{\frac{d \theta_{1}^{*}}{d \xi}\left[u_{1}^{*}\left(\bar{u}_{1}{ }^{2}+\bar{v}_{1}{ }^{2}\right)+\overline{2 u_{1}{ }^{*} p_{1}{ }^{*}}\right]\right\} \\
& +\left[2|A|^{2} k_{7}+\frac{2}{R_{\theta}}|A|^{2} k_{10} y=0\right.
\end{aligned}
$$

where $\left(k_{1} *+k_{2}^{*}\right)$ is the sum of the unnormalised integrals at each station. Let us write,

$$
k^{*}=\left(k_{1}^{*}+k_{2}^{*}\right)
$$

From our calculations for the mean velocity profile we have the values of $\theta_{1}{ }^{*}, \theta_{2}^{*}$ and $\theta^{*}$. Thus we may write

and

$$
\begin{aligned}
& \left.\theta_{1}^{*}=\lambda \theta^{*}\right) \\
& \left.\theta_{2}^{*}=(1-\lambda) \theta^{*}\right)
\end{aligned}
$$

Making use of expressions (7.58) and (7.59) we may write the three integral equations in their final form,

$$
\begin{aligned}
& \frac{1}{4}\left[-\left(U_{1} *-U_{2}^{*}\right)^{2}+4 \beta_{2}+4|A|^{2}\left(k_{1}-k_{2}\right)+4 \theta *|A|^{2}\left(\frac{d k_{1}}{d \theta^{*}}-\frac{d k_{2}}{d \theta^{*}}\right)-\right. \\
& \left.\frac{4}{k^{*}}|A|^{2}\left\{(1-\lambda)\left(\bar{u}_{2}{ }^{2}-\bar{v}_{2}{ }^{2}\right)+\lambda\left(\bar{u}_{1}{ }^{2}-\bar{v}_{1}^{*}\right)\right\}\right] \frac{d \theta^{*}}{d \xi}+\left[\theta *\left(k_{1}-k_{2}\right)\right] \\
& \frac{d|A|^{2}}{d \xi}+\left[V_{2}^{* U_{1} *}-U_{2}^{*} V_{1}^{*}\right]=0,
\end{aligned}
$$




$$
\begin{aligned}
& \frac{1}{8}\left[8 \beta_{3}+4\left(U_{1}^{*}+U_{2}^{*}\right) \beta_{2}-2\left(U_{1}^{*}-U_{2}^{*}\right)^{2} \beta_{1}-\left(U_{1} *-U_{2}^{*}\right)^{2}\left(U_{1} *+U_{2}^{*}\right)\right. \\
& +8|A|^{2}\left\{\left(k_{3}-k_{4}+k_{6}\right)+\theta^{*}\left(\frac{d k_{3}}{d \theta^{*}}-\frac{d k_{4}}{d \theta^{*}}\right)\right\}+\frac{8}{k^{*}}|A|^{2}\{(1-\lambda) \text {. } \\
& \left.\left.\left(\bar{u}_{2} *^{2}-\bar{v}_{2}{ }^{2}\right)-\lambda\left(\bar{u}_{1}{ }^{2}-\bar{v}_{1}{ }^{2}\right)\right\}\right] \frac{d \theta^{*}}{d \xi}+\left[\theta *\left(k_{3}-k_{4}\right)\right] \frac{d|A|^{2}}{d \xi}+ \\
& {\left[|A|^{2} k_{5}\right] \frac{d b^{*}}{d \xi}-\left[|A|^{2} k_{7}-\frac{1}{R_{\theta}} B_{4}\right]=0} \\
& {\left[|A|^{2}\left\{\frac{\left(U_{1}^{*}+U_{2}^{*}\right)}{2}\left(k_{1}+k_{2}\right)+\left(k_{3}+k_{4}-2 k_{6}+2 k_{8}\right)\right\}+|A|^{2}\right.} \\
& \theta *\left\{\frac{\left(U_{1}^{*}+U_{2}^{*}\right)}{2}\left(\frac{d k_{1}}{d \theta^{*}}+\frac{d k_{2}}{d \theta^{*}}\right)+\left(\frac{d k_{3}}{d \theta^{*}}+\frac{d k_{4}}{d \theta^{*}}+\frac{2 d k_{8}}{d \theta^{*}}\right\}-\frac{|A|^{2}}{k^{*}}\right. \\
& \left.\left\{(1-\lambda)\left[\left(\bar{u}_{2}{ }^{2}+\bar{v}_{2}{ }^{2}\right) U_{2}^{*}+\overline{2 u_{2}^{*} p_{2}^{*}}\right]+\lambda\left[\left(\bar{u}_{1}{ }^{2}-\bar{v}_{1}{ }^{2}\right) U_{1}^{*}+\overline{2 u_{1}^{*} p_{1}}\right]\right\}\right] \frac{d \theta^{*}}{d \xi} \\
& +\left[\theta *\left\{\frac{\left(U_{1}^{*}+U_{2}^{*}\right)}{2}\left(k_{1}+k_{2}\right)+\left(k_{3}+k_{4}+2 k_{8}\right)\right\}\right] \frac{d|A|^{2}}{d \xi}- \\
& {\left[2|A|^{2} k_{5}\right] \frac{a b^{*}}{d \xi}+\left[2|A|^{2} k_{7}+\frac{2}{R_{\theta}}|A|^{2} k_{10}\right]=0}
\end{aligned}
$$

Equations $(7.60),(7.61)$ and $(7.62)$ are first order differential equations for the three unknowns, $\theta^{*}, b^{*}$ and $|A|^{2}$. In the next section we will describe the calculation of the integral functions, $k_{i}$

\subsection{Calculation of the Integral Functions}

In order to evaluate the integral functions the Orr-Sommerfeld equation $(7.50)$ is solved and the distributions of fluctuating 
components are calculated from the eigensolutions. The measurements of Sato (1959) show that the natural frequency of the fundamental mode of the fluctuating field is $2.42 \mathrm{~Hz}$. Whe Reynolds number used is $4.2374 \times 10^{5}$ which corresponis to a reference plate length of $150 \mathrm{~mm}$. With the frequency fixed at this value the eigenvalues and eigenfunctions of the Orr-Sommerfeld equation may be determined as functions of the momentum thickness, $\theta *$. The amplification factor, $-\alpha_{i}{ }^{*}$, is shown as a function of $\theta^{*}$ in Figure 43. The measurements of Sato (1956, 1959) were made for the separated shear layer, i.e. $U_{2} *=0$, thus our calculatiors have been made for one stream stationary. Figure 43 shows that the maximum amplification occurs at a thickness $\theta^{*}$ of approximately .0175. The distribution across the shear layer of $\bar{u}^{2}$ shows the features of the disturbance profile measured by Sato (1959), Freymuth (1966) and Browand (1966). We may see that the distribution has a sharp minimum at $y / \theta^{*}=1.4$ which ccrresponds to a change in phase of the $\bar{u}^{*}-f l u c t u a t i o n$. The integral functions are then evaluated from the eigensolutions of the orr-Somerfeld equation and are normalised such that

$$
\int_{-n_{2}}^{n_{1}}\left(\bar{u}^{*^{2}}+\bar{v}^{2}\right) d n=k_{1}+k_{2}=1
$$

The integral functions are then known functions of $\theta^{*}$ and the three differential equations for $\theta^{*}, b^{*}$ and $|A|^{2}$ may be solved as an initial value problem.

\subsection{Results and Discussion}

The solution of equations $(7.60),(7.61)$ and $(7.62)$ presents 
a tedious computational problem. However we shall see below that by appropriate simplification of the differential equations much useful information about the influence of finite amplitude disturbances on the growth of the mixing layer may be obtained.

Firstly we will assume that the disturbance functions are zero outside the limits of integration. We have seen from our previous chapter on the turbulent problem that agreement between experiment and theory in, for instance, the case of the $\bar{u}^{* 2}$ distribution is good over the centre of the layer but, whereas the predicted profile exhibits two peaks outside this region the experimental results do not. Secondly, we will simplify the energy equations so as only to consider the kinetic energy of the disturbances and to disregard the $\xi$-derivatives of certain shape parameters. We know from the measurements of Browand (1966) that the value of $b^{*}$ is small and that, after a period of development, which will be influenced by the experimental situation, the value of $\frac{d b^{*}}{d \xi}$ is small. Thus we will write the three differential equations as,

$$
\begin{aligned}
\frac{d}{d \xi}\left[\left(\beta_{2}-1\right) \theta^{*}+|A|^{2}\left(k_{1}-k_{2}\right)\right] & =-2 V_{2}^{*}=\frac{-\gamma_{1}}{\sqrt{\xi}} \cdot \\
\frac{d}{d \xi}\left[\theta^{*}\left(\beta_{3}+\beta_{2}-\beta_{1}-1\right)\right] & =|A|^{2} k_{7}-\frac{\beta_{4}}{R \cdot \theta^{*}}, \\
\frac{d}{d \xi}\left[\theta^{*}|A|^{2} \cdot\left(k_{1}+k_{2}\right)\right] & =2|A|^{2} k_{7}-\frac{2|A|^{2} k_{10}}{R \cdot \theta^{*}}
\end{aligned}
$$

We may see from equation (7.64) that when $|A|^{2} \rightarrow 0$, i.e. for laminar flow, then $\frac{d \theta^{*}}{d \xi} \propto \frac{1}{\sqrt{\xi}}$ or, 


$$
\theta * \gamma_{3} \sqrt{\xi}
$$

In equations (7.65) the left hand side represents the derivative with $\xi$ of the energy of the mean flow. The value of $k_{r}$ is always negative and we can see that the disturbed flow is extracting energy from the mean flow. In equation (7.66) the left hand side represents the derivative with $\xi$ of the energy of the disturbed flow. It may be seen from the right hand side of this equation that the shear stresses are feeding energy to the disturbances whilst the viscous forces are applying damping. Combining equations $(7.65)$ and $(7.66)$ we obtain,

$\frac{1}{|A|^{2}} \frac{d|A|^{2}}{d \xi}=-\frac{k_{1}}{\theta^{*}}\left\{2-\frac{|A|^{2}}{\gamma_{2}}\right\}-\frac{1}{R_{0} \theta * *^{2}}\left\{2 k_{10}+\frac{4}{\gamma_{2}}\right\}$,

where $\gamma_{2}=1+\beta_{1}-\beta_{2}-\beta_{3}$.

Rearranging this equation we may write

$$
\begin{aligned}
& \frac{a|A|^{2}}{d \xi}=a_{1}|A|^{2}-a_{2}|A|^{4}-\frac{a_{3}}{R}|A|^{2} \\
& \text { where } a_{1}=\frac{-2 k_{7}}{\theta^{*}}, a_{2}=\frac{-k_{7}}{\theta^{*} r_{2}} \text { and } a_{3}=\frac{1}{\theta^{*}}\left\{2 k_{10}+\frac{4}{r_{2}}\right\}
\end{aligned}
$$

Equation (7.69) is to be compared with equation (2.15) of Stuart (1958) for the temporal growth of the disturbance amplitude. In our case the values of $a_{1}, a_{2}$ and $a_{3}$ are functions of $\xi$. It can be seen that when the disturbance magnitude is of order $\mathrm{R}^{-\frac{1}{2}}$ then the disturbance terms may no longer be considered as negligible. 
We may also note that the first two terms on the right hand side of equation (7.69) are of opposite sign which means there will be a value of $|A|^{2}$ for which $\frac{d|A|^{2}}{d \xi} \rightarrow 0$. From equation $(7.68)$ it is clearer that this value is given by $|A|^{2}=2 \gamma_{2}$. Having evaluated $k_{i}$ as a function of $\theta^{*}$ we will now solve equations $(7.64)$ and $(7.68)$ simultaneously to evaluate the growth of $\theta^{*}$ and $|A|^{2}$ with $\xi$. The required inputs to the computer solution are the starting values of $\xi, \theta^{*}$ and $|A|^{2}$. A number of different values of parameters have been considered and their effect noted. The equations are solved using a Runge-Kutta integration scheme and Iinear interpolation to evaluate the coefficients at each point. The starting value of $\xi$ was set at .01 , which corresponds to $x=1.5 \mathrm{~mm}$ and $\theta^{*}$ was started at a value of .004 , which corresponds to a value of $\theta$ of $6 \mathrm{~mm}$. The growth of the disturbance amplitude, $|A|^{2}$ is shown in Figure 45 for a number of different initial values of $|A|^{2}$. As is to be expected the smaller the initial value of $|A|^{2}$ the further downstream will the sudden growth occur. It may also be seen that the amplitude limit is reached as $|\mathrm{A}|^{2} \rightarrow 1.257$ the value of $2 \gamma_{2}$ being 1.2628, The effect of the initial disturbance amplitude on the growth of the mixing layer thickness, $\theta^{*}$ is shown in Figure 46. Once again the smaller the initial magnitude of the disturbance the longer will be the transition. We may also note that the smaller the initial amplitude of the disturbances the smoother is the transition. Up to the point of sudden growth of the mixing layer the rate of growth of $\theta^{*}$ is inversely proportional to ${ }^{\prime} \xi_{\text {o }}$ 
The two quantities measured by Sato with which

comparisons may be made are the value of $e^{*}$ and the energy density of the $\overrightarrow{\mathrm{u}}^{*}$-fluctuation defined by,

$$
E_{\theta}=\int_{-\infty}^{\infty} \overline{*^{2}} d n
$$

This function is shown in Figure 47 for various initial disturbance amplitudes. The maximum value reached is approximately .75 for all initial values of $|A|^{2}$. The experimental results of Sato give a value in the region of .6 , however in the case of the wake there existed a possible integration error of $10 \%$ 。 We may also note that in Sato's experiments the sub-harmonic of the fundamental occurred at a position short of the longitudinal point at which the maximum amplitude was reached. The distance over which the energy density grew from zero to a maximum value in Sato's measurements appears independent of the Reynolds number based on the plate length and free stream velocity. In our calculations the equivalent non-dimensional distance is given by $\xi \simeq .1$ which when dimensionalised with respect to $L^{*}=150 \mathrm{~mm}$ gives $15 \mathrm{~mm}$. The value given by Sato is up to $75 \mathrm{~mm}$ and thus we see that the rapid growth of the disturbances is faster in our calculations. It is clear that some mechanism is required to damp the growth of the solutions of the simplified set of equations $(7.64)$ and (7.68). Before examining the effect of the terms omitted from the complete equations let us look at the effect of increasing the viscous dissipation. In order to do this we will examine the effect on $\mathrm{E}_{\theta}$ of increasing and decreasing the Reynolds number.

The effect on the growth of $\mathrm{E}_{\theta}$ for four different values 
of Reynolds number is shown in Figure 48. The effect is similar to that caused by varying the initial value of the disturbance amplitude. This can be clearly seen from equation $(7, y)$ where decreasing the Reynolds number has the effect of increasing the disturbance magnitude in the viscous dissipation term. However the lower Reynolds number has a continuing effect through the transition and reduces the eventual equilibrium value of the energy density. For a reduction of order 10 in the Reynolds number the equilibrium value of the energy density decreases from 0.75 to 0.61 . This latter value agrees more closely with the measurements of Sato, however the transition length is still too small in comparison with measurements. There is a slight increase in the transition Iength as the Reynolds number is decreased. The use of a variable Reynolds number to isolate the effect of viscous dissipation may be used as at high Reynolds numbers the disturbance distribution integrals will be nearly independent of Reymolds number and will approach the inviscid Iimit。

Let us consider the possible influence of the terms omitted in our simplification of the energy equations. The term omitted from the left hand side of the fluctuating energy equation is

$$
\frac{d}{d \xi}\left[\theta *|A|^{2}\left(k_{3}+k_{4}+2 k_{8}\right)\right]
$$

From the calculated values we find that in the range $0<\theta^{*}<$ .025 the value of $k_{8}$ is nearly constant and equals -.25. The sum of $k_{3}$ and $k_{4}$ is also nearly constant in this range and is 
approximately equal to .4 . Thus the effect of this term is a factor of 10 smaller than the kinetic energy terms $k_{1}$ and $k_{2}$ 。 The term omitted from the left hand side of the mechanical energy equation is

$$
\begin{aligned}
& \frac{d}{d \xi}\left[\theta *|A|^{2}\left(k_{3}-k_{4}\right)\right]=\left[|A|^{2}\left\{\theta \frac{d\left(k_{3}-k_{4}\right)}{d \theta^{*}}+\left(k_{3}-k_{4}\right\}\right] \frac{d \theta^{*}}{d \xi}\right. \\
& +\theta *\left(k_{3}-k_{4}\right) \frac{d|A|^{2}}{d \xi} .
\end{aligned}
$$

As $\theta^{*}=.015$ which for $|A|_{\xi_{0}}^{2}=1 \times 10^{-6}$ is in the centre of the fast-growing region, the value of $\left(k_{3}-k_{4}\right)$ is zero. $a\left(k_{3}-k_{4}\right) / d 0^{*}$ is negative and it is clear that this term will increase the value of $\gamma_{2}$ which will lead to a higher limiting amplitude for the disturbance. However, it will also lead to a decrease in the rate of growth of the momentum thickness. The terms omitted from the right hand side of the equation represent the integrated product of $\frac{\partial U}{\partial x}$ and $\left(\bar{u}^{* 2}-\bar{v}^{2}\right)$. Experimental results show that $\bar{u}^{*}$ and $\bar{v}^{2}$ are nearly equal in the mixing layer and as the value of $\partial U / \partial x$ will be small those terms are unlikely to affect the solution.

The phase velocity of the disturbances given by $c_{r}=\omega_{r} / \alpha_{r}$ is shown in Figure 49. The measurements of Browand (1966) give the phase velocity as $1.16 \pm .22$, in our calculations the phase velocity varies between 1.7 and 1.1 . There appears to be no consistency in the measurements of Browand as to the relationship between phase velocity and longitudinal distance. The measurements of Sato for the wake show a sharp change in the phase 
velocity from the laminar to the turbulent regions.

Let us return to the amplitude growth of the disturbances. The experiments of Freymuth (1966) show that the growth rate of the main peak in the $\bar{u}^{*}$ fluctuation is independent of the applied sound pressure and increases ten times in a distance of approximately $30 \theta_{\mathrm{m}}$ where $\theta_{\mathrm{m}}$ is the momentum thickness of the layer at the separation point. Our definition of $\theta^{*}$ is different from that of Freymuth, the relation between the two being $\theta^{*}=4 \theta$. Thus our calculations should show a tenfold increase in a distance of approximately $8 \theta_{0}^{*}$. With $\theta_{0}^{*}=.004$ this distance is .032, and, as is shown on our Figure 45 for a value of $|A|_{0}^{2}=$ $1 \times 10^{-6}$ the distance is .038 . The same growth distance is found for the other values of initial disturbance magnitude. However this calculation may only be used as a quantitative check on the growth rate as calculations performed with different values of $\theta_{0}^{*}$ gave similar growth distances suggesting that the rate of growth is independent of the initial thickness. The smaller the initial thickness, the longer the longitudinal distance before transition. This may be seen in a plot of momentum thickness against $\xi$ for various values of $\theta_{0}^{*}$ in Figure 50. A decrease in the value of $\theta_{0}{ }^{*}$ results in an initial decrease in the Reynolds number based on momentum thickness and, as may be seen from Eqn. ( 7.65$)$, a consequent increase in the value of the viscous dissipation term.

\subsection{Conclusions}

We have seen how the development of the mixing layer from laminar to turbulent flow may be determined from a simplified set 
of momentum and energy equations. The growth of the mixing layer is shown to be proportional to $\xi^{\frac{1}{2}}$ for infinitessimal disturbances。 The point of transition occurs at a point which is a function of the initial amplitude of the disturbances, the flow Reynolds number and the initial momentum thickness. A decrease in Reynolds number leads to a lower limiting value for the energy density. The amplitude of the disturbances is shown to grow very rapidy and reaches a limiting value which, in the simplified equations, is a function of the mean velocity integrals. The limiting magnitude of the disturbances does not occur at a value of $\theta *$ corresponding to the neutral mode as was the case for the wake, Ko, Kubota and Lees (19'70). The rate of growth of the disturbance amplitude was shown to be of the same order as the measurements of Freymuth (1966) but independent of the initial thickness of the layer. The results of Sato (1956), however, indicate a rate of growth of the energy density less than our calculated values. The Iimiting value of energy density is a function of the Reynolds number and agreement between Sato's value and the calculations occurs for a value of $R$ of one-tenth of the real value. The maximum amplitude of the energy density is independent of the initial values of disturbance magnitude and momentum thickness. The phase velocity of the disturbances decreases from a value of 1.7 to a value of 1.1 through the transition region. 


\section{Conclusions}

The following conclusions may be drawn from our study of the laminar and turbulent mixing of two parallel streams which has been considered in four separate sections.

The analysis of the mean equations of laminar mixing may be solved numerically using an iterative solution. No assumptions, other than the normal boundary layer assumptions are required. For the solution of the mean turbulent mixing problem assumptions have to be made as to the description of the Reynolds stresses. It is found that the assumption of an eddy viscosity connecting Reynolds stresses to mean shear may be successfully employed. An exponential distribution of eddy viscosity of the form $v_{T}=v_{0} \exp \left(-A_{\xi}^{2}\right)$ is used, where $\xi$ is a similarity co-ordinate. A value of the constant $A$ of 0.7 is found to give good agreement between measured and calculated velocity profiles. The assumption that the eddy viscosity distribution is symmetric about a point corresponding to the position of maximum shear gives better results than an assumption of symmetry about the $\mathrm{x}$ axis. The distribution of eddy viscosity coincides with the distribution of intermittency which indicates that the eddy viscosity may be considered constant in regions of the flow which are fully turbulent. The relationship between turbulent Reynolds number and velocity ratio of the two streams is found on the basis of the size of eddies in various shear flows. The same analysis leads to a relationship between rate of spread of the layer and velocity ratio the result of which agrees with the analysis of Abramovitch. The ratio of maximum value of eddy viscosity to kinematic viscosity is taken from experimental results and is a function of velocity ratio. The similarity form 
of the momentum equation is solved numerically, using the above assumptions, and the results agree with experimental results for cases of zero and finite velocity of one stream.

The stability of the mixing layer between two streams may be studied by considering the growth of small disturbances in the laminar flow. The equation for the calculation of stability is formed by considering the triple Fourier transform of the fluctuating equations of momentum and continuity. The solution of the resulting boundary value problem determines whether disturbances will grow or decay. The stability problem may be posed as either a spatial or temporal one. In the former case disturbance frequency is real and wavenumber and eigenvalue are complex. In the temporal case the wavenumber is real and the frequency and eigenvalue are complex. The results of the analysis show that the laminar shear layer is unstable, in both spatial and temporal senses, to infinitesimal disturbances at all Reynolds numbers. This result is found to be a consequence of the assumption of parallel flow. It is found that for the case of two streams having finite velocity the results of spatial and temporal analyses lead to similar results for eigenfunctions and amplification factors. It may be shown that the case of one stationary stream is a special case of the general problem of the mixing of two streams and that as the velocity of the two streams tends to an equal value the results of spatial and temporal analyses coincide. A correction to the amplification factors for non-parallel flow may be made by considering the interaction energy between mean flow and disturbances. The resulting correction factor is found to be a function of Reynolds number and 
the corrected values of amplification lead to a critical Reynolds number of 12.3 .

The structure of the turbulent mixing layer is examined on the basis of a linear model. This provides a useful guide to the structure of the flow. The calculated convection and phase velocities agree with available experimental results. The rate of growth or decay of eddies as a function of wavenumber follows the trend exhibited in the boundary layer where the larger eddies decay more slowly than the smaller. For the shear layer the higher wavenumber disturbances grow more slowly than the lower. The fluctuating velocity field is predicted from the eigensolution of the homogeneous equation for turbulent shear flow corresponding to the fastest growing mode. The distribution of longitudinal velocity fluctuation agrees with experimental results but the transverse velocity fluctuation exhibits a trough at the centre of the layer and is a factor of two smaller than experimental results. The same feature is exhibited by the distribution of linear fluctuating pressure. It is shown that the effect of obliquity of the waves only reduces the magnitude of the transverse velocity fluctuation. It is shown that at the critical layer where the phase velocity equals the local mean velocity the non-linear terms in the equations of motion may not be neglected. It may be argued that the non-linear term will force a form of isotropy on the fluctuating field. The calculated distributions of shear stress and pressure diffusion agree well with measured or indirectly measured experimental values. The distribution of the shear correlation coefficient is nearly a constant value of 
unity across the mixing layer. The reason for this high yalue is the antiphase of $u$ and $v$ velocity fluctuations predicted by linearised theory. The pressure diffusion predicted by linear analysis is close to the expected experimental results. The linear analysis serves as a guide to those components of the turbulence governed by non-linearity.

The development of the mixing layer is examined from a simplified solution of equations of momentum and energy. The mean and fluctuating components of the flow may be expressed in terms of a number of shape parameters. The initial growth of the mixing layer is proportional to the square root of longitudinal distance. The point of transition from laminar to turbulent flow is a function of the initial amplitude of the disturbances, the initial momentum thickness and the flow Reynolds number. The amplitude of the disturbances grows very rapidly to reach a limiting value which is a function of integrals of the mean flow. The rate of growth of the disturbances predicted follows experimental results in one instance but the measurements are not conclusive. The limiting value of energy density is predicted to be higher than measured results though a reduction in flow Reynolds number reduces the predicted value. The phase velocity falls from a value of 1.7 to 1.1 through the transition region.

This work has indicated those processes in turbulent flow governed by linear and non-linear processes. The role of the nonlinear terms is shown as both amplifying and amplitude limiting. The work may be seen to be readily extendable to compressible flows which will only add to the complexity of the equations to be solved. 
It is to be hoped that an analysis of the sort presented above will aid the understanding of turbulence and ita effects. The application to noise generation by turbulence of this analysis presents an interesting problem. 


\section{APPENDIX I}

Discussion of the Third Boundary Condition for the Mixing of Two

\section{Parallel Streams.}

We have noted in our analysis in Chapter 3 that the equation for the stream function describing the mixing of two parallel streams is of the third order. It will thus require three boundary conditions. Two of these are immediately appaxent, namely that the $x$-component of velocity must reach that of the free stream on each side of the mixing layer,

$$
\begin{aligned}
& \mathrm{U} \rightarrow \mathrm{U}_{1} \text { as } \mathrm{y} \rightarrow \infty \\
& \mathrm{U} \rightarrow \mathrm{U}_{2} \text { as } \mathrm{y} \rightarrow-\infty,
\end{aligned}
$$

where $U_{1}$ and $U_{2}$ are the velocities of the upper and lower streams respectively. The third boundary condition necessary for the complete solution of the third-order differential equation has been considered by a number of workers. Kuethe (1935) made use of the third boundary condition proposed by von Karman based on the balancing of transverse momentum, though Kuethe made no attempt to justify this proposition. The most complete discussion, of the proper determination of the third boundary condition, so far presented was by Ting (1959). Ting made use of matched asymptotic expansions to derive the appropriate boundary conditions for compressible and incompressible mixing. In his paper he found it necessary to match second order pressures across the mixing layer, for the case of incompressible mixing, in order to derive the third boundary condition. It 
is shown below that this was not necessary and was due to an analytical error in Ting's paper.

Let us first consider a laminar mixing region and write the velocity components and pressure in the free stream above the mixing layer as series in powers of $\varepsilon$ where $l / \varepsilon$ is of the order of the square root of the Reynolds number.

$$
\begin{aligned}
& \left.U_{I}(x, y)=U_{S}\left\{\left(U_{1} / U_{S}\right)+\varepsilon U_{I}^{(I)}(x / L, y / L)+\varepsilon^{2} U_{I}^{(2)}(x / L, y / L)+\ldots\right\} \text { (AI. } 3\right) \\
& V_{I}(x, y)=U_{S}\left\{\varepsilon V_{I}^{(I)}(x / L, y / L)+\varepsilon^{2} V_{I}^{(2)}(x / L, y / L)+\ldots .0\right\} \\
& P_{1}(x, y)=P_{0}\left\{I+\varepsilon p_{1}^{(I)}(x / L, y / L)+\varepsilon^{2} p_{1}^{(2)}(x / L, y / L)+\ldots\right\} \\
& \text { where, } U_{S}=\frac{U_{1}+U_{2}}{2}, I \text { is a reference length, } \varepsilon^{2}=\frac{v_{0}}{U_{S} L_{1}} \text {, } \\
& v_{0} \text { is the kinematic viscosity of the parallel streams } \\
& \text { and } \mathrm{P}_{0} \text { is the pressure of the parallel streams. }
\end{aligned}
$$

The corresponding expansions for the lower stream are,

$$
\begin{aligned}
& U_{2}(x, y)=U_{S}\left\{\left(U_{2} / U_{S}\right)+\varepsilon U_{2}{ }^{(I)}(x / L, y / L)+\varepsilon^{2} U_{2}^{(2)}(x / L, y / L)+\ldots\right\} \\
& V_{2}(x, y)=U_{S}\left\{\varepsilon V_{2}^{(I)}(x / L, y / L)+\varepsilon^{2} V_{2}{ }^{(2)}(x / L, y / L)+\ldots\right. \\
& P_{2}(x, y)=P_{0}\left\{I+\varepsilon P_{2}{ }^{(I)}(x / L, y / L)+\varepsilon^{2} P_{2}{ }^{(2)}(x / L, y / L)+\ldots\right.
\end{aligned}
$$

In the preceding expansions the subscripts 1 and 2 refer to quantities in the upper and lower streams respectively and the superscripts define the order of the term in the series expansion in powers of $\varepsilon$.

The flow outside the mixing layer is irrotational and inviscid and we may write Bernoulli's equation for the upper 
stream in terms of the series (AI.3, 4, 5),

$$
\begin{aligned}
\frac{P_{0}}{\rho_{0}}+\frac{U_{1}^{2}}{2}= & \frac{P_{0}}{\rho_{0}}+\frac{\varepsilon P_{0} P_{I}(1)}{\rho_{0}}+\frac{\varepsilon^{2} P_{0} P_{I}(2)}{\rho_{0}}+\ldots \\
& \left.+\frac{U_{S}^{2}}{2}\left\{\left(\frac{U_{I}}{U_{S}}\right)^{2}+\frac{2 \varepsilon U_{I} U_{I}(I)}{U_{S}}+\varepsilon^{2} U_{I}(1)^{2}+\frac{2 \varepsilon^{2} U_{I} U_{I}(2)}{U_{S}}+\ldots\right\}\right\} \\
& +\frac{U_{S}^{2}}{2}\left\{\varepsilon^{2} V_{I}(I)^{2}+\ldots 00000\right\}
\end{aligned}
$$

where $\rho_{0}$ is the density of the two parallel streans.

Collecting coefficients of like powers of $\varepsilon$ we obtain,

$$
\begin{aligned}
& \frac{P_{0}}{\rho_{0}}+\frac{U_{I}^{2}}{2}=\frac{P_{0}}{\rho_{0}}+\frac{U_{I}^{2}}{2} \\
& P_{I}{ }^{(I)}=-\left\{\frac{\rho_{0} U_{I} U_{S}}{P_{0}}\right\} U_{1}{ }^{(I)}(x / L, y / L), \\
& \text { (AI.IO) } \\
& P_{I}{ }^{(2)}=-\frac{\rho_{0} U_{S}^{2}}{2 P_{0}}\left\{\left[U_{I}{ }^{(I)}\right]^{2}+\left[V_{I}(I)^{2}\right\}-\left\{\frac{\rho_{0} U_{I} U_{0}}{P_{0}} U_{I}(2)(x / L, y / L),(A I . I I)\right.\right.
\end{aligned}
$$

Similar expressions will exist for the pressure terms in the lower stream. We note in particular that

$P_{2}{ }^{(I)}=-\left\{\frac{\rho_{0} U_{2} S_{S}}{P_{0}} U_{2}{ }^{(I)}(x / L, y / L)\right.$

It can be seen from equations (AI.10) and (AI.I2) that the first order pressures on each side of the layer are proportional to the product of axial velocity and the first order $x$-component of velocity. It will be shown that the first order $x$-component of velocity is a function of the transverse velocity at the edge of the layer and in this way the boundary condition proposed by von Karman is satisfied. 
Let us expand the velocity components and pressure inside the mixing layer as follows.

$\mathrm{U}(\mathrm{x}, \mathrm{y})=\mathrm{U}_{\mathrm{s}}\left\{\mathrm{U}^{(0)}(\mathrm{x}, \mathrm{Y})+\varepsilon \mathrm{U}^{(\mathrm{I})}(\mathrm{x}, \mathrm{Y})+\ldots\right\}$

$V(x, y)=\varepsilon U_{S}\left\{V^{(0)}(x, Y)+\varepsilon V^{(1)}(x, Y)+\ldots\right\}$

and

$P(x, y)=P_{0}\left\{P^{(0)}(X, Y)+\varepsilon P(I) \quad(X, Y)+\ldots\right\}$

where $Y=y / \varepsilon$ is the stretched comordinate inside the mixing layer。

The relationships between quantities within the layer and those in the flow above the mixing layer are,

$\lim _{Y \rightarrow \infty} U^{(0)}(x / L, Y / L)=U_{I} / U_{0}$
$\operatorname{Lim}_{Y \rightarrow \infty} U^{(I)}(x / L, Y / L)=U_{1}{ }^{(I)}(x / L,+0)$

$\operatorname{Lim}_{Y \rightarrow \infty} V^{(0)}(x / L, Y / L)=V_{I}^{(I)}(x / L,+0)$

and

$\operatorname{Lim}_{Y \rightarrow \infty} P^{(0)}(x / L, Y / L)=I$

$\operatorname{Lim} P^{(I)}(x / L, Y / L)=P_{I}{ }^{(I)}(x / L,+0)$ 
The corresponding relationships between the quantities inside the layer and those in the flow below the mixing layer are,

$\operatorname{Lim}_{Y \rightarrow+\infty} U^{(0)}(x / L, Y / L)=U_{2} / U_{O}$

$\operatorname{Lim}_{Y \rightarrow \rightarrow \infty} U^{(I)}(x / L, Y / L)=U_{2}^{(I)}(x / L,-0)$

$\operatorname{Lim}_{Y \rightarrow \infty} V^{(0)}(x / L, Y / L)=V_{I}{ }^{(1)}(x / L,-0)$

and

$\operatorname{Lim}_{Y \rightarrow-\infty} P(0)(X / L, Y / L)=I$

$\operatorname{Lim}_{Y \rightarrow-\infty} P^{(I)}(x / L, Y / L)=P_{2}^{(I)}(x / L,-0)$

Substituting the series (AI.13, 14, 15) into the Navierm Stokes equations and collecting coefficients of like powers of $\varepsilon$ then we obtain from the $y$-component equation,

$$
\begin{gathered}
\frac{\partial P}{\partial Y}^{(0)}=0, \\
\frac{\partial P}{\partial Y}^{(1)}=0, \\
\text { and } U^{(0)} \frac{\partial V}{\partial X}^{(0)}+V^{(0)} \frac{\partial V}{\partial Y}=-{\frac{P O}{\rho_{O} U_{S}^{2}}}^{(0)} \frac{\partial P}{\partial Y}+\frac{\partial^{2} V^{(0)}}{\partial Y^{2}}
\end{gathered}
$$


Then equation (AI.27), together with the matching conditions (AI.20) and (AI.25), leads to,

$$
\mathrm{P}_{1}{ }^{(I)}(\mathrm{x} / \mathrm{I},+0)=\mathrm{P}_{2}^{(I)}(\mathrm{x} / \mathrm{L},-0)
$$

Equation (AI.29) states the condition that there is no net first order pressure difference across the mixing layex. This compatability condition serves to provide the necessary third boundary condition.

In a linear theory we may represent the shear layer as a distribution of sinks lying on the $x$-axis. Then according to thin aerofoil theory we have,

$$
\mathrm{U}^{(I)}-i V^{(I)}=\frac{I}{\pi} \int_{0}^{\infty}\left\{V^{(0)} \frac{\left(\omega / L_{2}, 0\right)}{(z-\omega)}\right\} d \omega,
$$

(see Tsien (1956)), where $z=x+i y$

In order to evaluate the integral in equation (AI.30) we will now introduce a stream function $\psi^{(0)}(x, y)$ given by,

$$
\psi^{(0)}(x, y)=\sqrt{x U_{S} \nu_{0}} f(n)
$$

where $n$ is a similarity variable given by,

$$
n+(Y / \sqrt{x I})-n_{0}
$$

The asymptotic behaviour of the stream function and the velocity components are,

$$
f(n) \rightarrow\left(\frac{U_{1}}{U_{S}}\right)(n+\alpha)
$$




$$
\begin{aligned}
& U^{(0)} \rightarrow U_{I}, \\
& n \rightarrow+\infty \\
& v^{(0)} \rightarrow \frac{1}{2} v \frac{v U_{S}}{x}\left(n_{0}-\alpha\right)\left(\frac{U_{1}}{U_{S}}\right), \\
& f(n) \rightarrow\left(\frac{U_{2}}{U_{S}}\right)(n+\beta), \\
& U^{(0)} \rightarrow U_{2} \text {, } \\
& \eta+\infty \\
& \text { and } \quad V^{(0)} \quad \frac{1}{2} y \cdot \frac{\nu U_{S}}{x}\left(n_{0}-\beta\right)\left(\frac{U_{2}}{U_{S}}\right) \text {, }
\end{aligned}
$$

where $\alpha$ and $\beta$ are known constants of the similar solution and the inclusion of $n_{0}$ permits $f^{8}(0)=1$.

Then for the flow above the mixing layer from (AI.30) and (AI.33) we have,

$$
U_{1}{ }^{(I)}-i V_{I}{ }^{(I)}=-\frac{U_{1} \alpha_{I}}{\pi} \int_{0}^{\infty} \frac{d \omega}{\sqrt{\omega}(z-\omega)},
$$

where $\alpha_{1}=-\frac{1}{2 U_{S}}\left(n_{0}-\alpha\right) \vee \frac{\nu_{0}}{U_{s}}$

The solution of equation $(A I .35)$ is

$$
U_{1}{ }^{(1)}-i V_{1}{ }^{(I)}=\frac{-i U_{1} \alpha_{1}}{\sqrt{z}}+\frac{U_{I} \alpha_{I}}{\pi \sqrt{z}} \log \left\{\frac{1+\sqrt{z}}{1-\sqrt{z}}\right\} \text {. }
$$

It is to be noted that in his analysis Ting only noted the singularity at $\omega=\mathrm{z}$ in the integrand of $(\mathrm{AI} .35)$ and not the singularity where $\sqrt{\omega}=0$. This led in the limit as $\mathrm{y} \rightarrow 0, U_{1}{ }^{(I)}=0$ as the right hand side of equation (AI.36) is then entirely imaginary. As $y \rightarrow+0$ we have from equation (AI.36) that 


$$
U_{1}^{(I)}\left(x / I_{1}+0\right)=\frac{U_{1} \alpha_{1}}{\pi \sqrt{x}} \log \left\{\frac{1+\sqrt{x}}{I-\sqrt{x}}\right\}
$$

Making use of equation (AI.10) with (AI.37) we obtain,

$$
P_{1}^{(J)}(x / L,+0)=-\left\{\frac{\rho_{0}^{U} s}{P_{0}}\right\} \frac{U_{1}^{2} \alpha_{1}}{\pi \sqrt{x}} \log \left\{\frac{1+\sqrt{x}}{1-\sqrt{x}}\right\}
$$

Similarly when $\mathrm{y} \rightarrow-0$ we may obtain,

$$
P_{2}{ }^{(I)}(x / L,-0)=\left\{\frac{\rho_{0} U_{s}}{P_{0}}\right\} \frac{U_{2}^{2} a}{\pi \sqrt{x}} \log \left\{\frac{I+\sqrt{x}}{1-\sqrt{x}}\right\}
$$

where $\alpha_{2}=-\frac{1}{2 U_{S}}\left(n_{0}-\beta\right) \sqrt{\frac{v_{0}}{U_{S}}}$.

Then from equations $(A I, 38)$ and $(A I, 39)$ with the compatability condition (AI.29) we have,

$$
\frac{U_{2}^{2} \alpha_{2}}{U_{s} \sqrt{x}}+\frac{U_{1}^{2} \alpha_{1}}{U_{s} \sqrt{x}}=0
$$

with equations (AI.33) and (AI.34) we may finally write this condition

$$
U_{1} V^{(0)}(x / L, \infty)+U_{2} V^{(0)}(x / L,-\infty)=0 \text { 。 }
$$

This equation constitutes the third boundary condition on our third-order differential problem. It may be seen that this agrees with the proposition of von Karman equation (3.2)。 However, it is to be noted that this condition is not necessarily correct for higher order matching of pressures across

$$
\text { A. I. } 8 \text {. }
$$


the mixing layer. We may also note that if we introduce an eddy viscosity solution for the turbulent mixing problem and then introduce an appropriate similarity factor we may also derive the result (AI.40) for the turbulent problem. 
APPENDIX II

The Use of Orthogonalisation Procedure in Numerical Solution of Boundary-Value Problems

In this section we will consider a simple procedure for the numerical solution of ill-conditioned boundary-value problems. Included in this class of problem is the solution of the OrrSommerfeld equation.

Let us firstly introduce the idea of orthogonalisation of vectors. A set of vectors are linearly dependent if they may be written,

$$
\sum_{m=1}^{n} \lambda_{m} v_{m}=0
$$

where $V_{m}$ are the vectors and $\lambda_{m}$ are arbitrary numbers at least one of which is non-zero. If this relation (A.II.I) is not satisfied then the vectors are said to be linearly independent. A system of unit vectors ${\underset{\sim}{n}}_{n}$ which satisfy the relation,

$$
\varepsilon_{i}^{2}=1, \quad e_{i} e_{j}=0, \quad i \neq j \text {, }
$$

form an orthogonal system. We may see that an orthogonal set is also linearly independent.

We may form an orthogonal set of vectors from any arbitrary system of Iinearly independent vectors $V_{i}$. Let us first set,

$$
e_{i}=v_{i} /\left|v_{i}\right|
$$

We require to find a scalar $\lambda_{i}$ such that $V_{j}-\lambda_{i} e_{i}$ and $e_{i}$ are orthogonal. It is clear that $\lambda_{i}$ is given by the dot 
product of $V_{j}$ and $e_{i}$, that is,

$$
\lambda_{i}=\underset{\sim j}{V}: e_{i}
$$

As $V_{j}$ and $e_{i}$ are linearly independent vectors we may construct a unit vector orthogonal to $e_{i}$ given by,

$$
e_{j}=\mid \frac{v_{j}-\lambda_{i} \cdot \dot{e}_{i}}{v_{j}-\lambda_{i} \cdot e_{i}}
$$

We may apply the same procedure to orthogonalise a set of linearly independent functions.

This technique may be applied to the numerical solution of boundary-value problems where the equation to be solved is illconditioned. We will now introduce the numerical technique and explain the main steps in the computer solution. A simple example of the technique rill then be given. Let us write two linearly independent solutions, and their $(n-1)$ derivatives, of an $n^{\text {th }}$ order linear differential equation as

$$
G_{1}(y)=\left(\begin{array}{c}
g_{1}(y) \\
g_{1}^{\prime}(y) \\
g_{1}^{\prime \prime}(y) \\
\vdots \\
g_{1}{ }^{-1}(y)
\end{array}\right) \quad \text { and } \quad g_{2}(y)=\left(\begin{array}{c}
g_{2}(y) \\
g_{2}^{\prime}(y) \\
g_{2}^{\prime \prime}(y) \\
\vdots \\
g_{2}^{n-1}(y)
\end{array}\right)
$$

The equation may be integrated from some linearly independent starting conditions to a value of $\mathrm{y}=\mathrm{y}_{1}$. Then applying the technique of orthogonalisation on the values of $G_{1}$ and $G_{2}$ at $y=y_{1}$ we obtain 


$$
E_{I_{1}}\left(y_{I}\right)=\frac{G_{I}\left(y_{1}\right)}{\left|\frac{d_{1}\left(y_{1}\right)}{G_{J_{1}}}\right|}
$$

and

$$
\left.\mathbb{L}_{2}\left(y_{1}\right)=\frac{G_{2}\left(y_{1}\right)-\left(G_{2}\left(y_{1}\right) \cdot E_{1}\left(y_{1}\right)\right) E_{1}\left(y_{1}\right)}{\left|G_{2}\left(y_{1}\right)-\left(G_{2}\left(y_{1}\right) \cdot E_{1}\left(y_{1}\right)\right) E_{1}\left(y_{1}\right)\right|}\right)
$$

The same transformations must then be applied to the initial conditions such that they now become,

and

$$
\begin{aligned}
& g_{1}(0)=\frac{G_{I}(0)}{\left|G_{I}\left(y_{I}\right)\right|}
\end{aligned}
$$

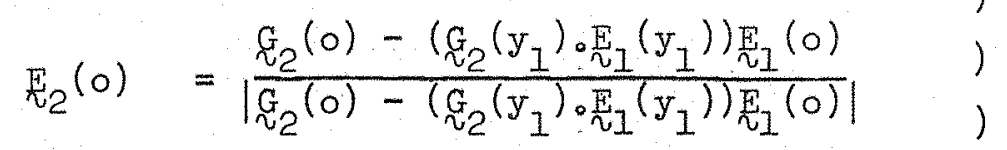

The integration may then procede from $y=y_{1}$ to $y=y_{2}$ with $\mathrm{i}_{1}\left(\mathrm{y}_{1}\right)$ and $\mathrm{Z}_{2}\left(\mathrm{y}_{1}\right)$ as the starting conditions. It must be remembered that the transformation applied to orthogonalise each set of vectors at each new step of the integration must be applied to each previous set of vectors.

The steps to be used in the computer solution using this technique may be written in sequence as follows,

(i) set up initial vectors $G_{1}(0)$ and $G_{2}(0)$,

(ii) integrate to position $\mathrm{y}=\mathrm{y}_{1}$,

(iii)create orthogonal vectors $\mathrm{E}_{2}\left(\mathrm{y}_{1}\right)$ and $\mathrm{i}_{2}\left(\mathrm{y}_{1}\right)$ at $\mathrm{y}=\mathrm{y}_{1}$,

(iv) perform same transformation on initial conditions to obtain $\mathrm{E}_{1}(0)$ and $\mathrm{E}_{2}(0)$,

(v) integrate from $\mathrm{y}=\mathrm{y}_{1}$ to $\mathrm{y}=\mathrm{y}_{2}$ with $\underset{\mathrm{N}}{\mathrm{E}}\left(\mathrm{y}_{1}\right)$ and $\mathrm{v}_{2}\left(\mathrm{y}_{1}\right)$ as initial conditions, 
(vi) create orthogonal vectors $\mathrm{E}_{1}\left(\mathrm{y}_{2}\right)$ and $\mathrm{i}_{2}\left(\mathrm{y}_{2}\right)$ at $\mathrm{y}=\mathrm{y}_{2}$, (vii) perform same transformations on vectors $E_{1}\left(y_{1}\right), E_{2}\left(y_{1}\right)$ and $\mathrm{E}_{1}(0), \mathrm{E}_{2}(0)$,

(viii)integrate from $y=y_{2}$ to $y=y_{3}$ with $\mathbb{2}_{1}\left(y_{2}\right)$ and $\mathbb{E}_{2}\left(y_{2}\right)$ as initial conditions, (ix) continue procedure until end of range of integration.

Let us now consider a simple example of the solution of an ill-conditioned differential equation which we will write as,

$$
\begin{aligned}
f^{i v}(y) & -24 f^{\prime \prime}(y)-169 f^{\prime \prime}(y)-324 f^{\prime}(y) \\
& -180 f(y)=0 .
\end{aligned}
$$

Its general solution is,

$$
f(y)=a_{1} e^{-y}+a_{2} e^{-2 y}+a_{3} e^{-3 y}+a_{4} e^{30 y}
$$

We will consider the solution with $a_{4}=0$ with boundary conditions

$$
f(0)=0, f^{\prime}(0)=0, f^{\prime \prime}(0)=2, f^{\prime \prime \prime}(0)=-12,
$$

so that the solution is,

$$
f(y)=e^{-y}-2 e^{-2 y}+e^{-3 y}
$$

The values of $f(y)$ and $f^{\prime}(y)$ at $y=1$ are then

and

$$
\begin{aligned}
& f(1)=.1470 \\
& f^{\prime}(1)=.0241
\end{aligned}
$$

We will now solve this equation $\left(A_{0} I I .9\right)$ as a boundary value problem with boundary conditions given by $f(0)=f^{\prime}(0)=0$ and conditions (A.II.13). Let us start the integration with conditions, 


$$
G_{1}(0)=\left|\begin{array}{l}
0 \\
0 \\
1 \\
0
\end{array}\right|
$$$$
\text { and } G_{2}(0)=\left|\begin{array}{c}
0 \\
0 \\
0 \\
1
\end{array}\right|
$$

Integrating from $y=0$ to $y=y_{1}$ we obtain,

$$
\begin{aligned}
& \left.g_{1}(1)=.196 \times 10^{10}, g_{I}^{\prime}(1)=.587 \times 10^{11},\right) \\
& g_{2}(1)=.326 \times 10^{4} \text { and } g_{2}^{\prime}(I)=.978 \times 10^{10} \text { ) }
\end{aligned}
$$

Now the solution of (A.II,9) is a linear combination of $G_{I}$ and $G_{2}$ such that

$$
f(y)=a g_{1}(y)+b g_{2}(y)
$$

' $a$ ' and ' $b$ ' may be calculated from (A.II.I5) using (A.II.4) and we then obtain,

$$
f^{\prime \prime}(0)=-.006
$$

and $f^{\prime \prime \prime}(0)=.036$.

which clearly is not the case. The Iinear independence of the originally linear independent șolutions has been contaminated by the solution with exponent 30 in equation (A.II.II). In order to ensure linear independence of the solutions we may use the orthogonalisation procedure outlined above. Integrating from $y=0$ to $y=I$ th a step size of $.0 I$ and performing the orthogonalisation after each integration step we finally obtain the result,

$$
\begin{aligned}
f^{\prime \prime}(0) & =-2.00000037 \\
\text { and } \quad f^{\prime \prime \prime}(0) & =17.9999908 .
\end{aligned}
$$


This accuracy may be further increased by a decrease in step size and a consequent increase in the possible number of orthogonalisations.

In the solution of the Orr-Sommerfeld equation, which is ill-conditioned in the above sense, the solutions are complex. No difficulty is experienced in FORTRAN $V$ programming as the functions may be declared as COMPLEX. However, care must be taken in forming the scalar operator $\lambda_{i}$ in orthogonalisation as for complex vectors it is the dot product of $V_{j}$ and the complex conjugate of $e_{i^{*}}$ (see equation (A.II.4)). 
REFERENCES

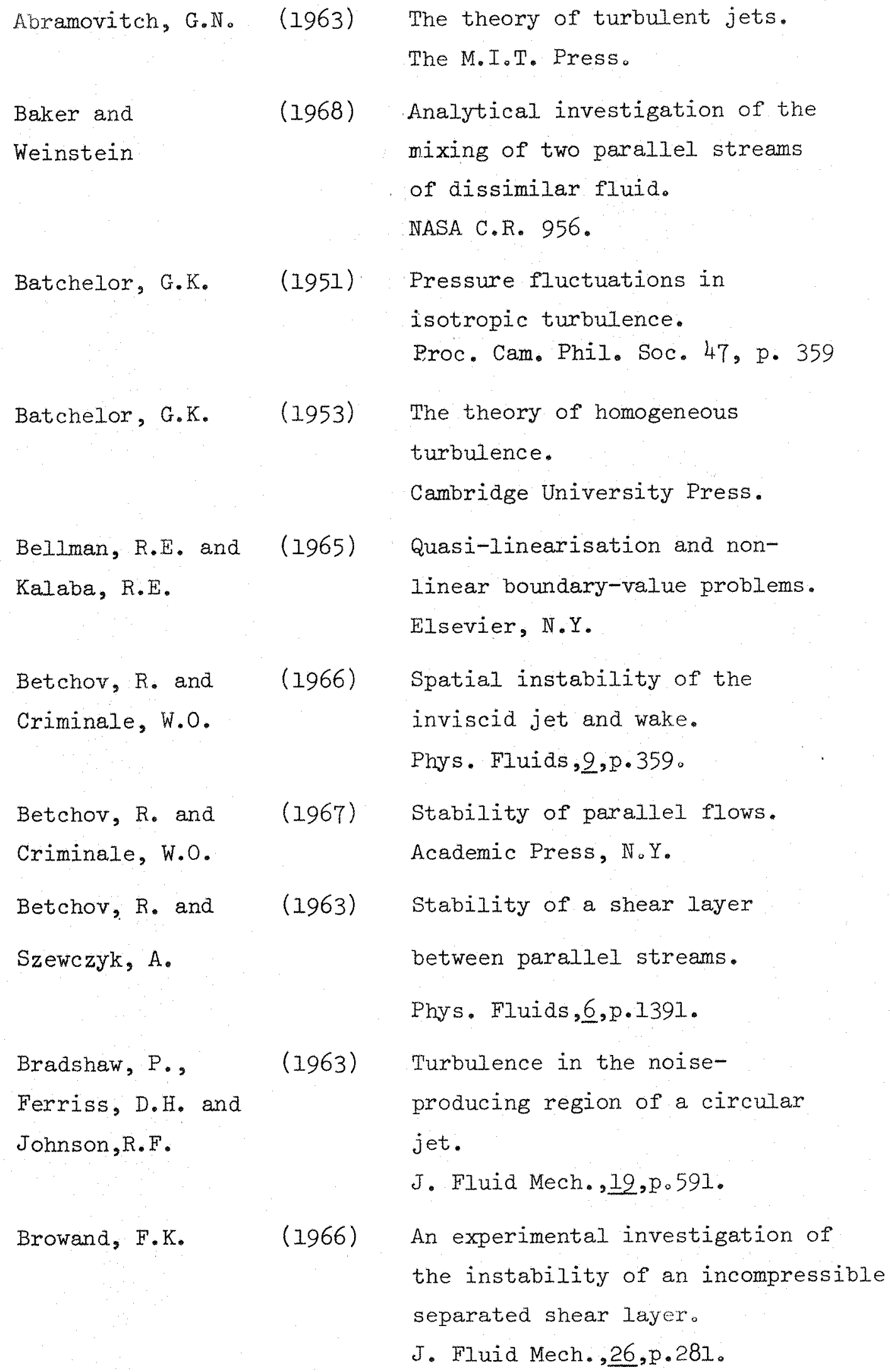




\begin{tabular}{|c|c|c|}
\hline Brown, W.Byron. & (1959) & $\begin{array}{l}\text { Numerical calculation of the } \\
\text { stability of cross-flow profiles. } \\
\text { Northrop Aircraft Inc., Rep.NAI 59-5 }\end{array}$ \\
\hline Chrandrasekhar, S. & (1961) & $\begin{array}{l}\text { Hydrodynamic and hydromagnetic } \\
\text { stability. } \\
\text { Oxford University Press. }\end{array}$ \\
\hline Corcos, G.M. & (1964) & $\begin{array}{l}\text { The structure of the turbulent } \\
\text { pressure field in boundary layers. } \\
\text { J. Fluid Mech., 18, p. } 353 \text {. }\end{array}$ \\
\hline $\begin{array}{l}\text { Corcos, G.M. and } \\
\text { Sellars, J.R. }\end{array}$ & (1959) & $\begin{array}{l}\text { On the stability of fully developed } \\
\text { flow in a pipe. } \\
\text { J. Fluid Mech. } 5, \mathrm{p} .97 \text {. }\end{array}$ \\
\hline Crane, L.J. & (1957) & $\begin{array}{l}\text { The laminar and turbulent mixing of } \\
\text { jets of compressible fluid. Part II- } \\
\text { the mixing of two semi-infinite streams. } \\
\text { J. Fluid Mech., } 3, p_{0} 81 \text {. }\end{array}$ \\
\hline $\begin{array}{l}\text { Davey, } \\
\text { Nguyen, }\end{array}$ & (1971) & $\begin{array}{l}\text { Finite-amplitude stability of pipe flow. } \\
\text { J. Fluid Mech. } 45, p .701 \text {. }\end{array}$ \\
\hline $\begin{array}{l}\text { Davies, P.O.A.L. and } \\
\text { Bose, B. }\end{array}$ & $(1968)$ & $\begin{array}{l}\text { Convection velocity measurements in } \\
\text { strong turbulence. } \\
\text { Univ. of Southampton, } \\
\text { I.S.V.R. Memo. } 225 \text {. }\end{array}$ \\
\hline $\begin{array}{l}\text { Davies, P.O.A.L., } \\
\text { Fisher, M.J. and } \\
\text { Barratt, M.J. }\end{array}$ & (1963) & $\begin{array}{l}\text { The characteristics of the turbulence } \\
\text { in the mixing region of a round jet. } \\
\text { J. Fluid Mech., I5, p. } 337 \text {. }\end{array}$ \\
\hline $\begin{array}{l}\text { Dolph, C.L. and } \\
\text { Lewis, D.C. }\end{array}$ & (1958) & $\begin{array}{l}\text { On the application of infinite systems } \\
\text { of ordinary differential equations to } \\
\text { perturbations of plane Poiseuille flow. } \\
\text { Quart. Appl. Math., } 16, p \cdot 97 \text {. }\end{array}$ \\
\hline Eagles, P.M. & (1969) & $\begin{array}{l}\text { Composite series in the Orr-Sommerfeld } \\
\text { problem for symmetric channel flow. } \\
\text { Quart. J. Mech. Appl., 22, p. } 129\end{array}$ \\
\hline
\end{tabular}




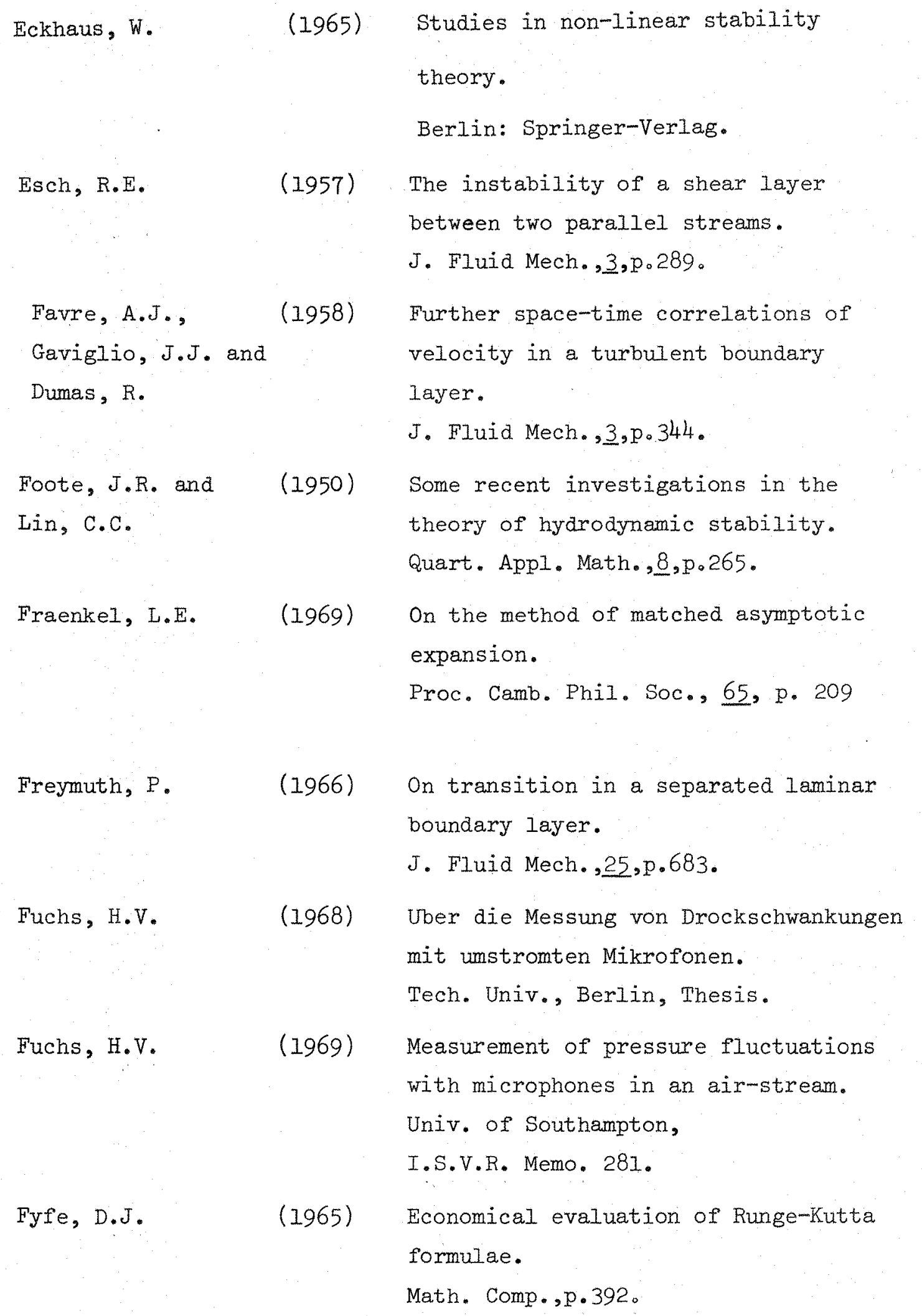




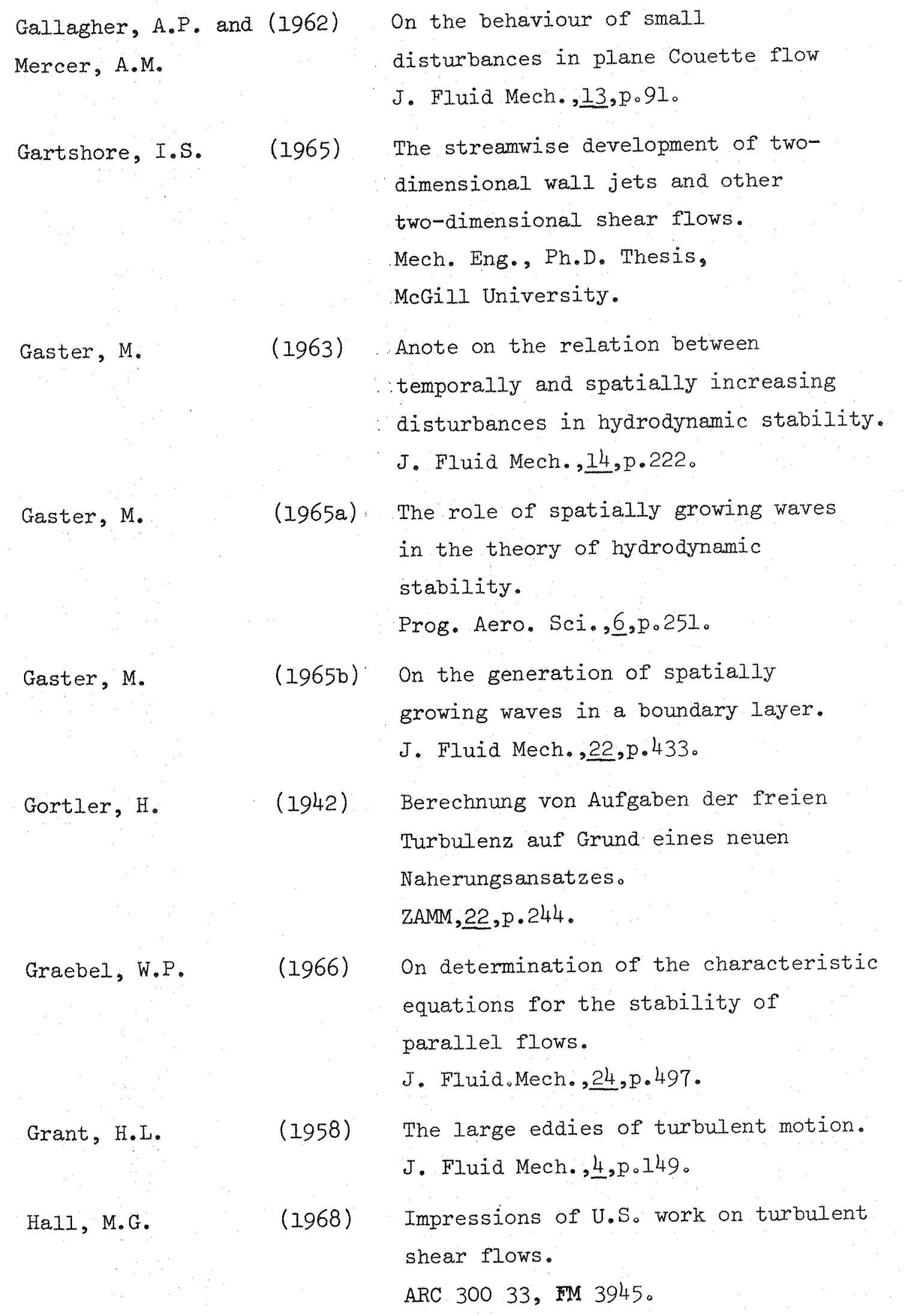




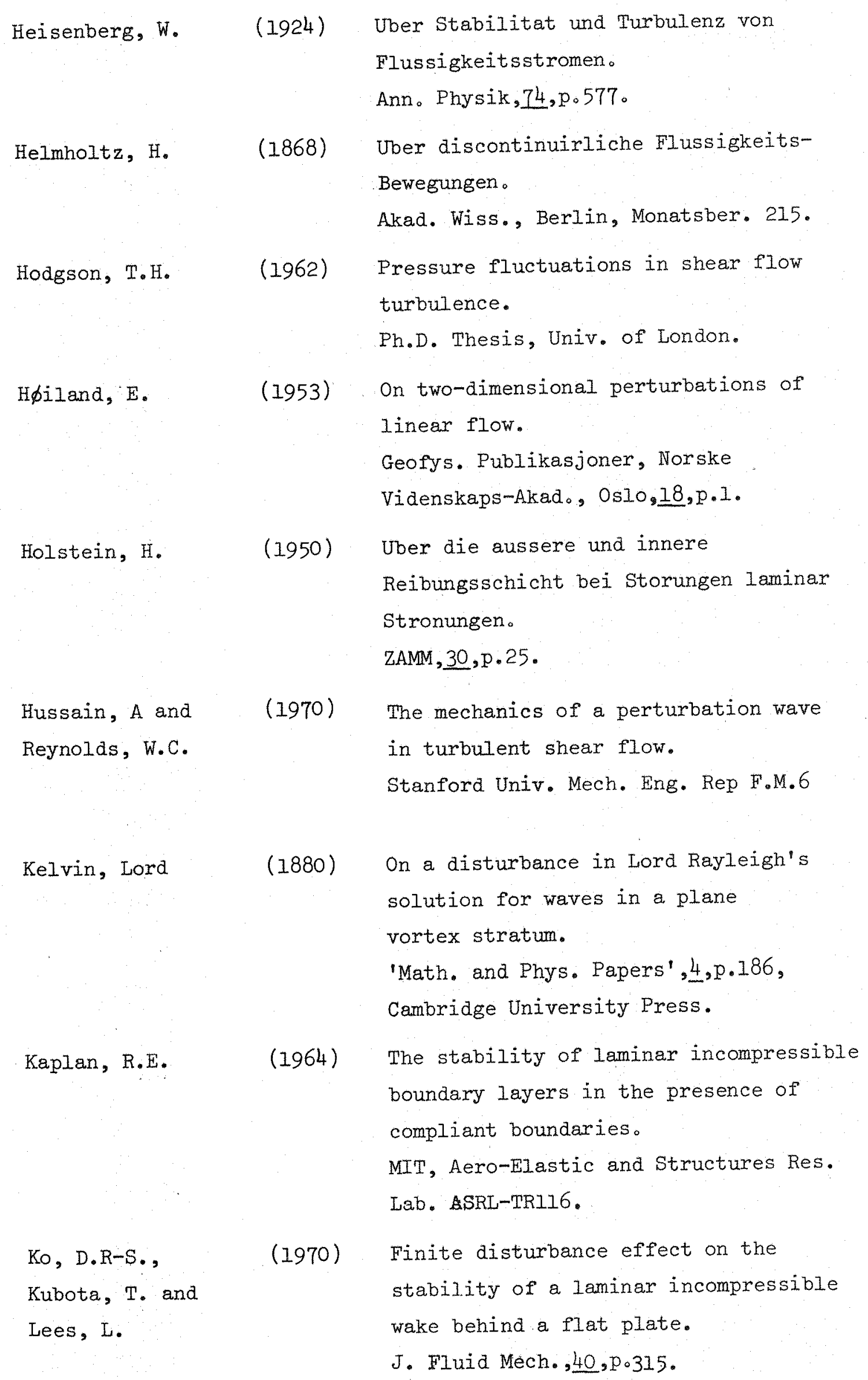




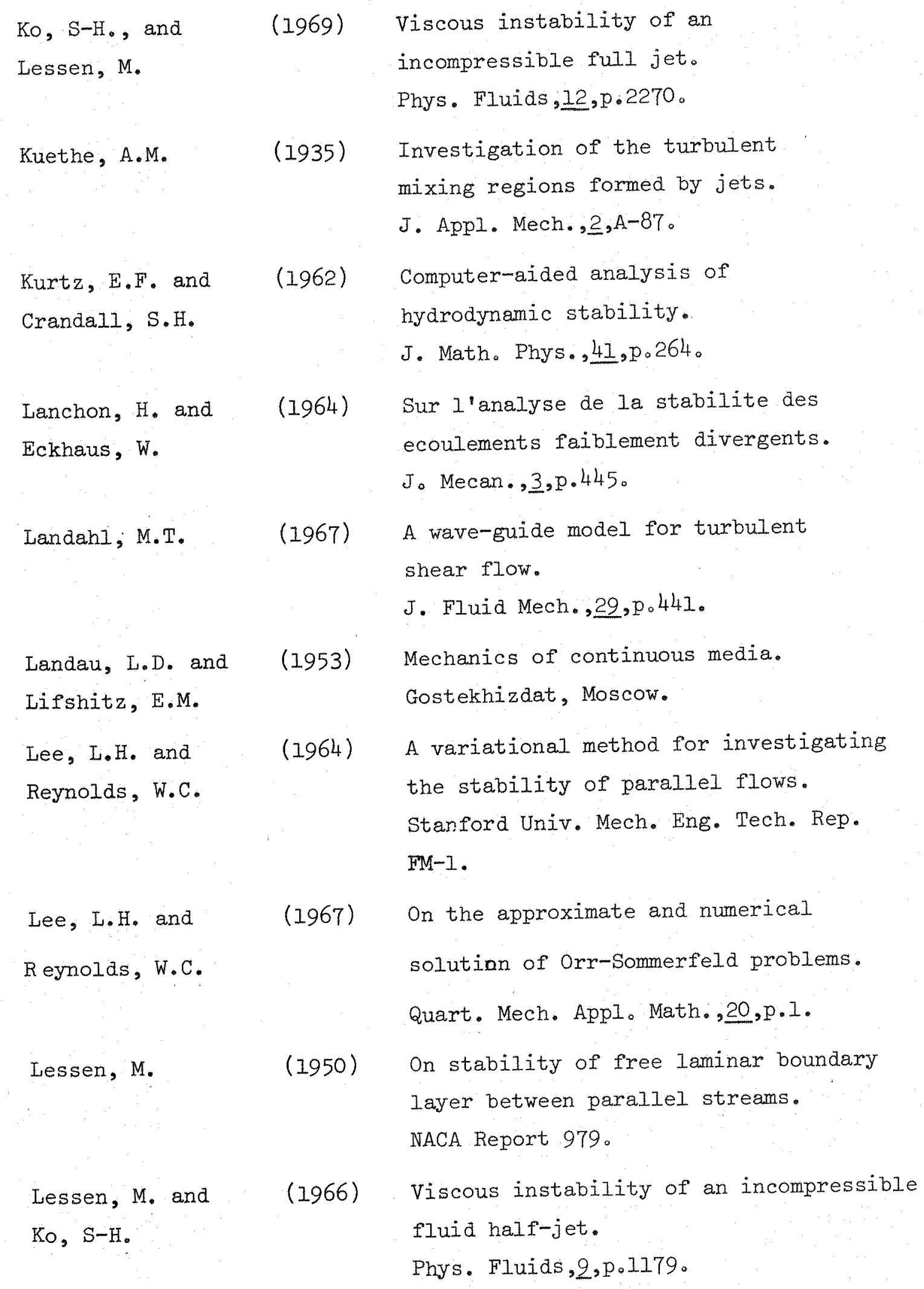




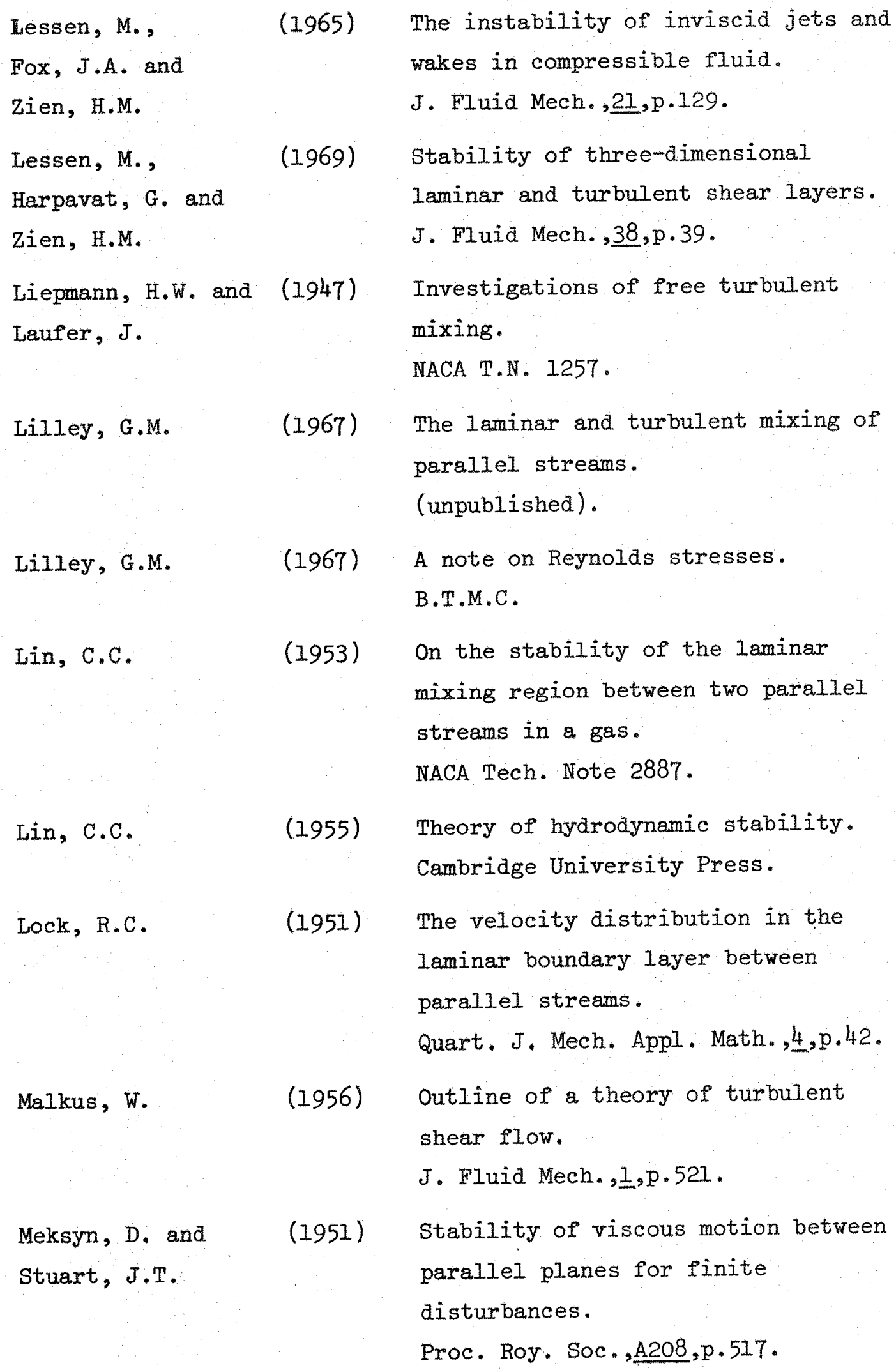

(1965) The instability of inviscid jets and wakes in compressible fluid.

J. Fluid Mech.,2l,p.129.

(1969) Stability of three-dimensional

laminar and turbulent shear layers.

J. Fluid Mech., 38, p.39.

(1947) Investigations of free turbulent

mixing.

NACA T.N. 1257.

(1967) The laminar and turbulent mixing of parallel streams.

(unpublished).

(1967) A note on Reynolds stresses.

B.T.M.C.

(1953) On the stability of the laminar mixing region between two parallel streams in a gas.

NACA Tech. Note 2887.

(1955) Theory of hydrodynamic stability. Cambridge University Press.

(1951) The velocity distribution in the laminar boundary layer between parallel streams. Quart. J. Mech. Appl. Math., 4, p.42. 


\begin{tabular}{|c|c|c|}
\hline Michalke, A. & (1964) & $\begin{array}{l}\text { On the inviscid instability of the } \\
\text { hyperbolic tangent velocity profile. } \\
\text { J. Fluid Mech.,19,p.543. }\end{array}$ \\
\hline Michalke, A. & (1965) & $\begin{array}{l}\text { On spatially growing disturbances in } \\
\text { an inviscid shear layer. } \\
\text { J. Fluid Mech., } 23, p .521 \text {. }\end{array}$ \\
\hline Michalke, A. & (1969) & $\begin{array}{l}\text { A note on spatially growing three- } \\
\text { dimensional disturbances in a free } \\
\text { shear layer. } \\
\text { J. Fluid Mech. } 38, \text { p. } 765 \text {. }\end{array}$ \\
\hline Michalke, A. & (1969) & $\begin{array}{l}\text { The influence of the vorticity } \\
\text { distribution on the inviscid } \\
\text { instability of a free shear layer. } \\
\text { 'Fluid Dynamics Transactions',4, } \\
\text { Polish Acad. Sci., Warsaw, p.751. }\end{array}$ \\
\hline Miles, J.W. & (1957) & $\begin{array}{l}\text { On the generation of surface waves } \\
\text { by shear flow. } \\
\text { J. Fluid Mech., } 3, p .185 \text {. }\end{array}$ \\
\hline Mills, R.D. & (1968) & $\begin{array}{l}\text { Numerical and experimental } \\
\text { investigations of the shear layer } \\
\text { between two parallel streams. } \\
\text { J. Fluid Mech.,33,p.59l. }\end{array}$ \\
\hline Moffat, H.K. & (1965) & $\begin{array}{l}\text { The interaction of turbulence with } \\
\text { rapid uniform shear. } \\
\text { Stanford Univ. Res. Rep., } \\
\text { Sudaer } 242 .\end{array}$ \\
\hline Newman, B.G. & (1967) & $\begin{array}{l}\text { Turbulent jets and wakes in a } \\
\text { pressure gradient. } \\
\text { 'Fluid Mechanics of Internal Flow', } \\
\text { ed. Sovran, Elsevier. }\end{array}$ \\
\hline Orr, W.McF. & (1907) & $\begin{array}{l}\text { The stability or instability of the } \\
\text { study of motions of a liquid. } \\
\text { Proc. Roy. Irish Acad.,A27, p.9. }\end{array}$ \\
\hline
\end{tabular}




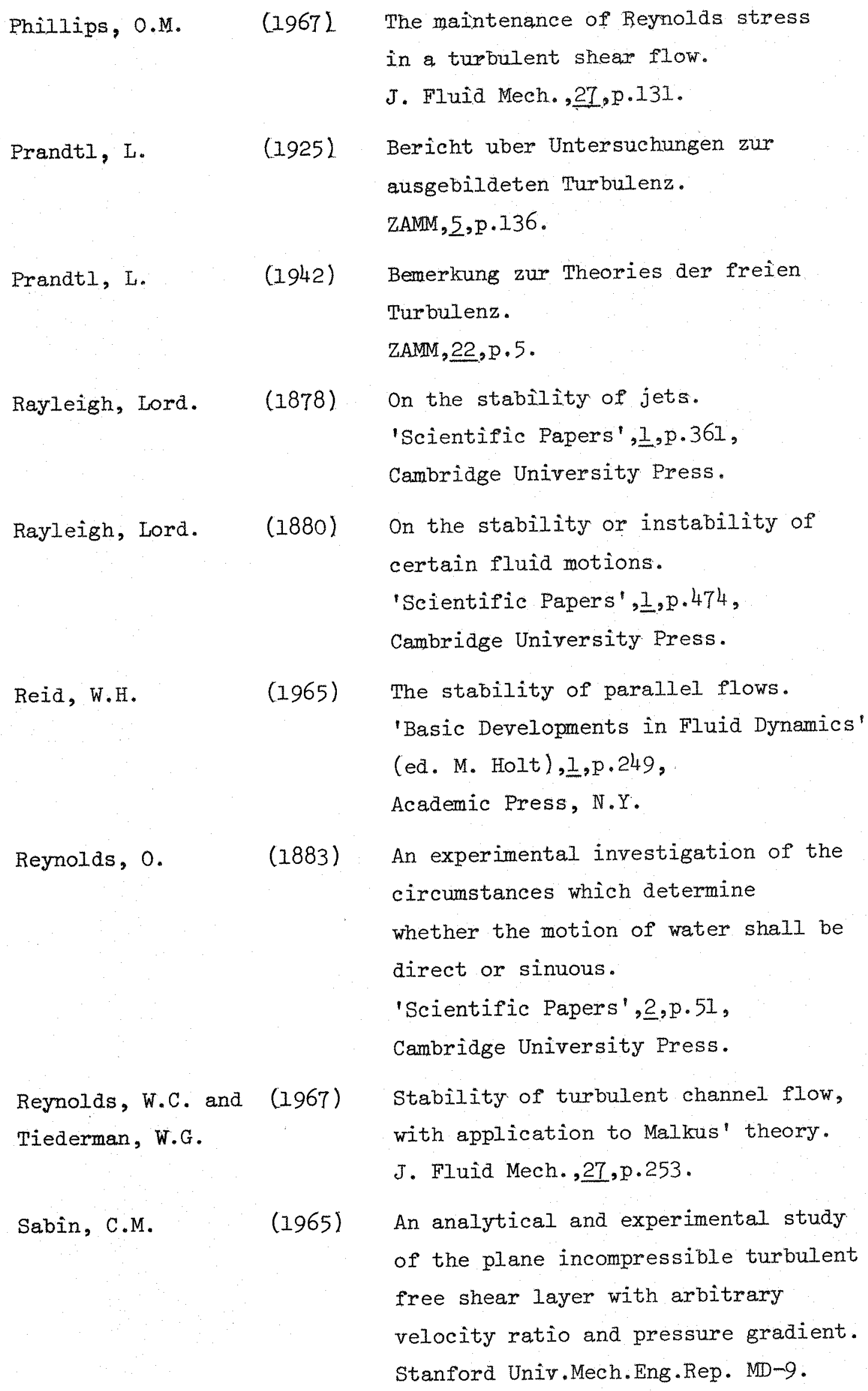




\begin{tabular}{|c|c|c|}
\hline Sato, H. & (1956) & $\begin{array}{l}\text { Experimental investigation on the } \\
\text { transition of the laminar separated } \\
\text { layer. } \\
\text { J. Phys. Soc. Japan,11,p.702. }\end{array}$ \\
\hline Sato, H. & (1959) & $\begin{array}{l}\text { Further investigation on the } \\
\text { transition of two-dimensional } \\
\text { separated layer at subsonic speeds. } \\
\text { J. Phys. Soc. Japan,14,p.1797. }\end{array}$ \\
\hline $\begin{array}{l}\text { Schade, H. and } \\
\text { Michalke, A. }\end{array}$ & $(1962)$ & $\begin{array}{l}\text { Formation of vortices in a free } \\
\text { boundary layer. } \\
\text { Z. Flugwiss., } 10, p \cdot 147\end{array}$ \\
\hline $\begin{array}{l}\text { Schubauer, G.B.and } \\
\text { Skramstad, H.K. }\end{array}$ & (1943) & $\begin{array}{l}\text { Laminar boundary layer oscillations } \\
\text { and transition on a flat plate. } \\
\text { NACA Tech. Rept. } 909 .\end{array}$ \\
\hline Sharma, R. & (1968) & $\begin{array}{l}\text { The structure of turbulent shear } \\
\text { flow. } \\
\text { Ph.D. Thesis, Univ. of Southampton. }\end{array}$ \\
\hline Sommerfeld, A. & $(1908)$ & $\begin{array}{l}\text { Ein Beitrag zur hydrodynamischen } \\
\text { Erklarung der turbulenten } \\
\text { Flussigkeitsbewegung. } \\
\text { Proc. 4th. Int. Congr. Maths, Rome, } \\
\text { p.116. }\end{array}$ \\
\hline Squire, H.B. & (1933) & $\begin{array}{l}\text { On the stability of three- } \\
\text { dimensional disturbances of viscous } \\
\text { flow between parallel walls. } \\
\text { Proc. Roy. Soc., A22l, p.189. }\end{array}$ \\
\hline Stuart, J.T. & (1958) & $\begin{array}{l}\text { On the non-linear mechanics of } \\
\text { hydrodynamic stability. } \\
\text { J. Fluid Mech., } 4, p .1 \text {. }\end{array}$ \\
\hline Stuart, J.T. & $(1960)$ & $\begin{array}{l}\text { On the non-linear mechanics of wave } \\
\text { disturbances in stable and unstable } \\
\text { parallel flows. I. The basic } \\
\text { behaviour in Poiseuille flow. } \\
\text { J. Fluid Mech., 2, p. } 353 \text {. }\end{array}$ \\
\hline
\end{tabular}


Stuart, J.T.

Tatsumi, T. and

Gotoh, K.

Taylor, G.I.

Thomas, L.H.

Ting, Lu.

Tollyien, W.

Tollmien, W.

Townsend, A.A.

Townsend, A.A.

Tsien, H.S.

Tu, B.J. and

Willmarth, W.W.
(1965) The production of intense shear layers by vortex stretching and convection. N.P.L. Aero. Rept. 1147.

(1960) The stability of free boundary layers between two uniform streams.

J. Fluid Mech., I,p.433.

(1935) Statistical theory of turbulence.

Proc. Roy. Soc., Al 51,p.42I.

(1953) The stability of plane Poiseuille flow. Phys. Rev.,91, p.780.

(1959) On the mixing of two parallel streams. J. of Math. Phys., 38,p.153.

(1926) Calculation of turbulent expansion processes.

ZAMM, 6, p. 468. also

NACA T.M. 1085 (1945).

(1929) Uber die Entstehung der Turbulenz. Gessellschaft der Wissenschaften, Gottingen, 21.

(1956) The structure of turbulent shear flow. Cambridge University Press.

(1962) Remarks on the Malkus theory of turbulent flow. Mechanique de la Turbulence, Paris: CNRS,p.167.

(1956) The Poincare-Lighthill-Kuo method. Advances in Appl. Mech., 4, p. 56.

(1966) An experimental study of the structure of turbulence near the wall. Aerospace Eng. Aero. Lab. Rept. 02920-3-T. Univ of Michigan. 


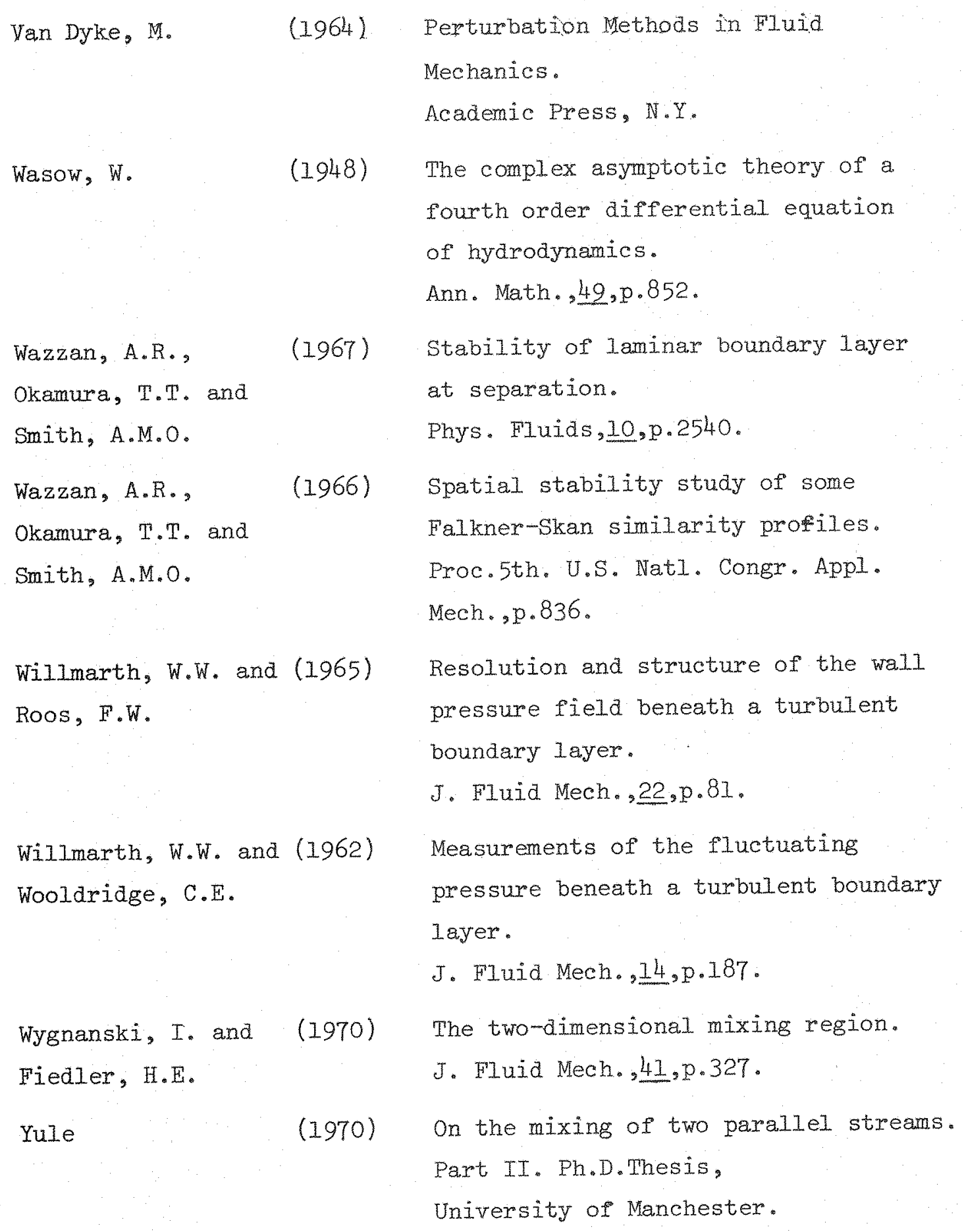




\begin{tabular}{|c|c|c|}
\hline Velocity Ratio $\frac{\mathrm{U}_{2}}{\mathrm{U}_{1}}$ & 0.0 & 0.61 \\
\hline$n=J / \frac{v x}{U_{s}}$ & $P^{\prime \prime}(n)$ & $f^{\prime}(n)$ \\
\hline-8 & -.9998 & -1.0 \\
\hline-7.2 & -.9972 & -.9999 \\
\hline-6.4 & -.9919 & -.9999 \\
\hline-5.6 & -.9815 & -.9994 \\
\hline-4.8 & -.9607 & -.9968 \\
\hline-4 & -.9196 & -.9854 \\
\hline-3.6 & -.8863 & -.9712 \\
\hline-3.2 & -.8404 & -.9462 \\
\hline-2.8 & -.7777 & -.9049 \\
\hline-2.4 & -.6934 & -.8407 \\
\hline-2 & -.5824 & -.7469 \\
\hline-1.6 & -.4406 & -.6185 \\
\hline-1.2 & -.2663 & -.4540 \\
\hline-0.8 & -.0628 & -.2574 \\
\hline-0.4 & .1594 & -.0389 \\
\hline 0.0 & .3825 & .1860 \\
\hline 0.4 & .5852 & .3995 \\
\hline 0.8 & .7490 & .5856 \\
\hline 1.2 & .8649 & .7341 \\
\hline 1.6 & .9360 & .8421 \\
\hline 20 & .9735 & .9136 \\
\hline 2.4 & .9905 & .9566 \\
\hline 2.8 & .9970 & .9800 \\
\hline 3.2 & .9992 & .9916 \\
\hline 3.6 & .9998 & .9968 \\
\hline 4 & .9999 & .9989 \\
\hline 4.8 & 1.0 & .9999 \\
\hline 5.6 & 1.0 & 3.0 \\
\hline
\end{tabular}

TABIE I

Laminar Velocity Distributions. 


\begin{tabular}{|c|c|c|}
\hline Velocity Ratio & 0.0 & 0.61 \\
\hline $\mathrm{l}=\mathrm{y} / \mathrm{L}(\mathrm{x})$ & $f^{\prime}(n)$ & $f^{\prime}(n)$ \\
\hline-2.0 & -.9999 & -.9999 \\
\hline-1.8 & -.9992 & -.9997 \\
\hline-1.6 & -.9920 & -.9938 \\
\hline-1.4 & -.9656 & -.9642 \\
\hline-1.2 & -.9039 & -.8933 \\
\hline-1.0 & -.7965 & -.7733 \\
\hline-0.8 & -.6393 & -.6158 \\
\hline-0.6 & -.4341 & -.4342 \\
\hline-0.4 & -.1874 & -.2389 \\
\hline-0.2 & .0883 & -.0369 \\
\hline O. & .3718 & .1671 \\
\hline 0.2 & .6317 & .3688 \\
\hline 0.4 & .8315 & .5611 \\
\hline 0.6 & .9479 & .7329 \\
\hline 0.8 & .9915 & .8687 \\
\hline 1.0 & .9995 & .9547 \\
\hline 1.2 & .9999 & .9916 \\
\hline 1.4 & 1.0 & .9994 \\
\hline 1.6 & 1.0 & 1.0 \\
\hline
\end{tabular}

\section{TABLE II}

Turbulent Velocity Distributions。 


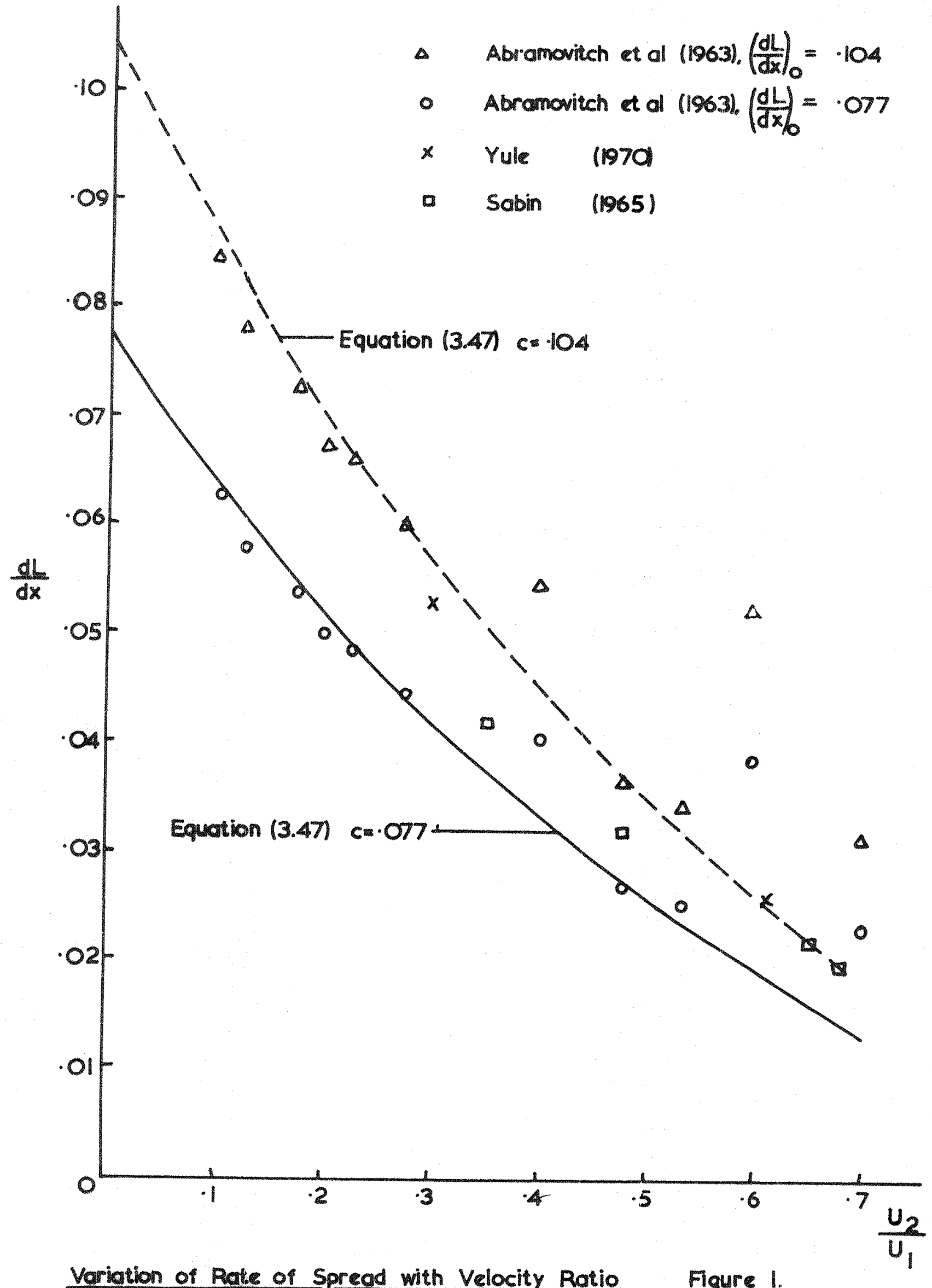




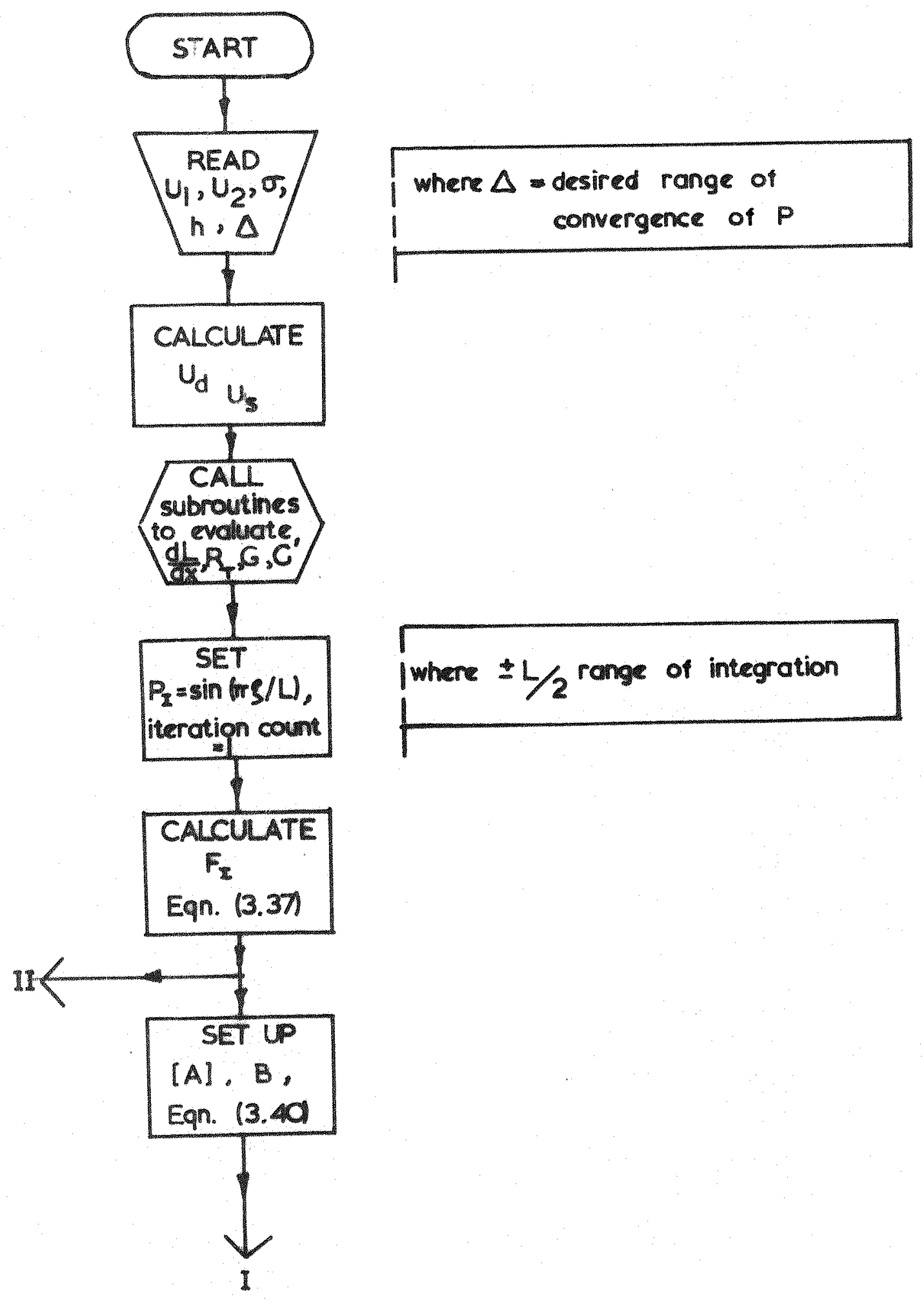

Flow Diggram of Program to Colculate Mean Velocity

Figure 2 Part I 


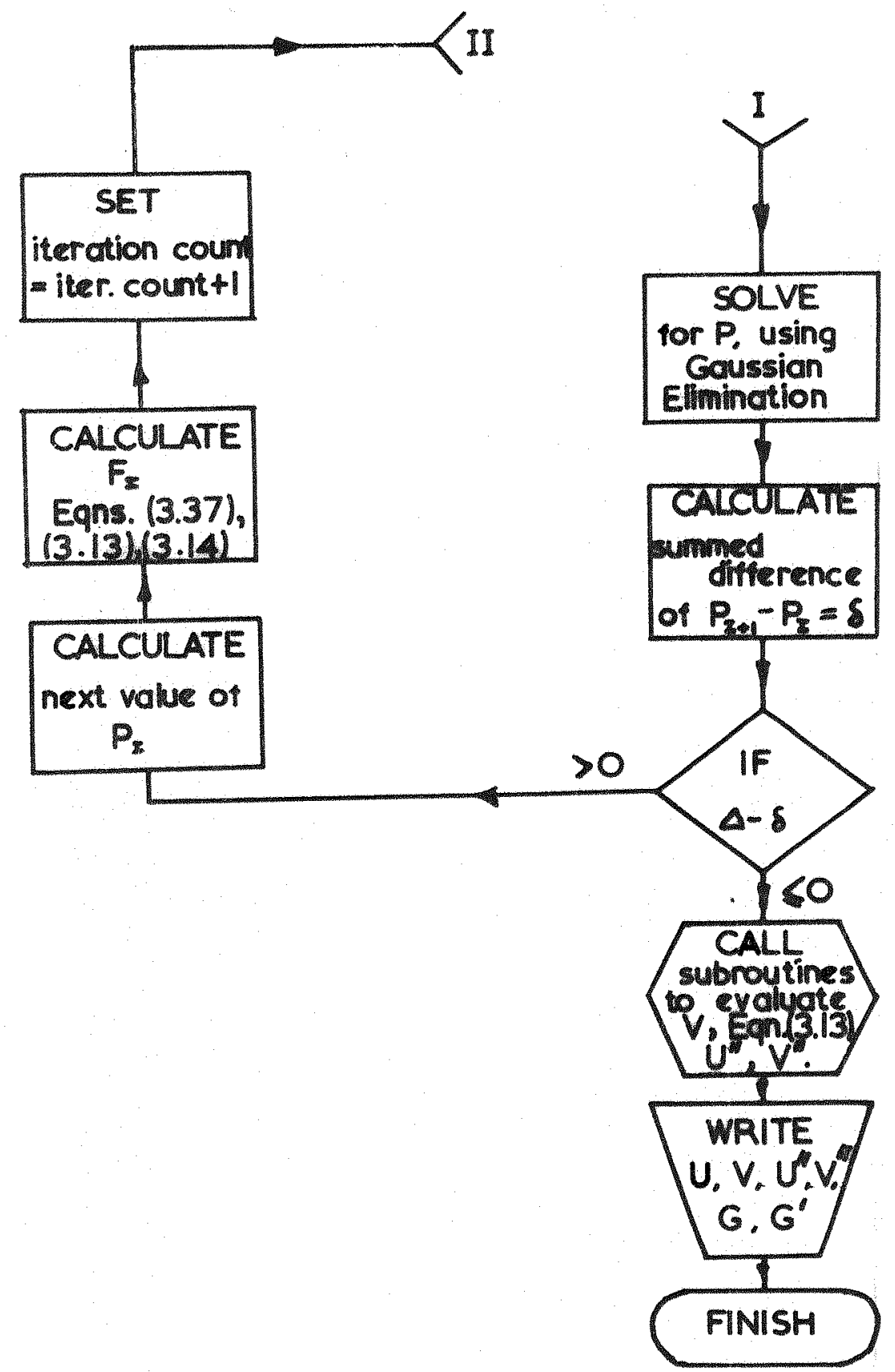

Flow Diagrum of Progran to Calculate Mean Velocity_ Figure 2 Part II 


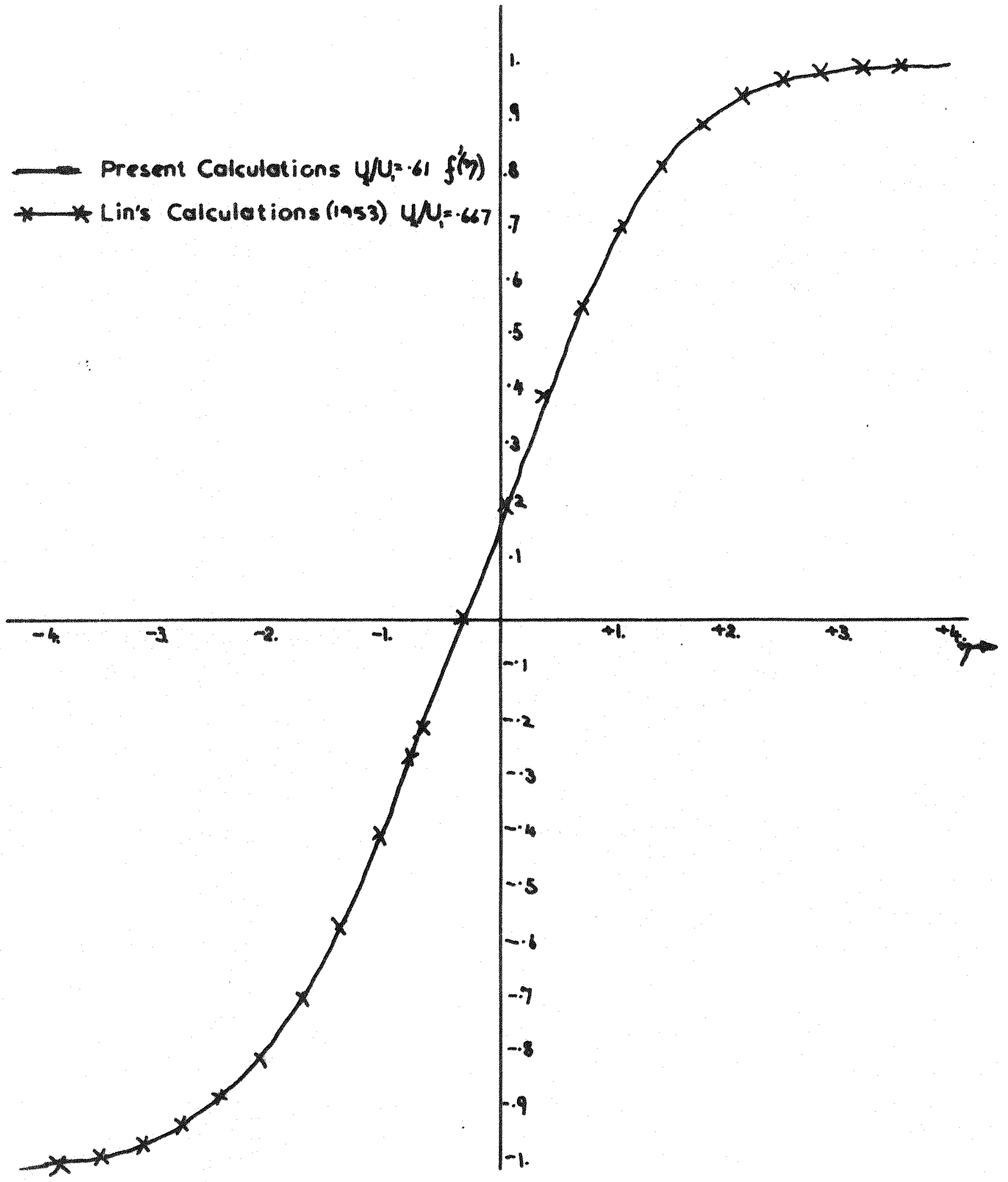

Nondimensional Laminar Velocity Prafile Fiqure 3. 


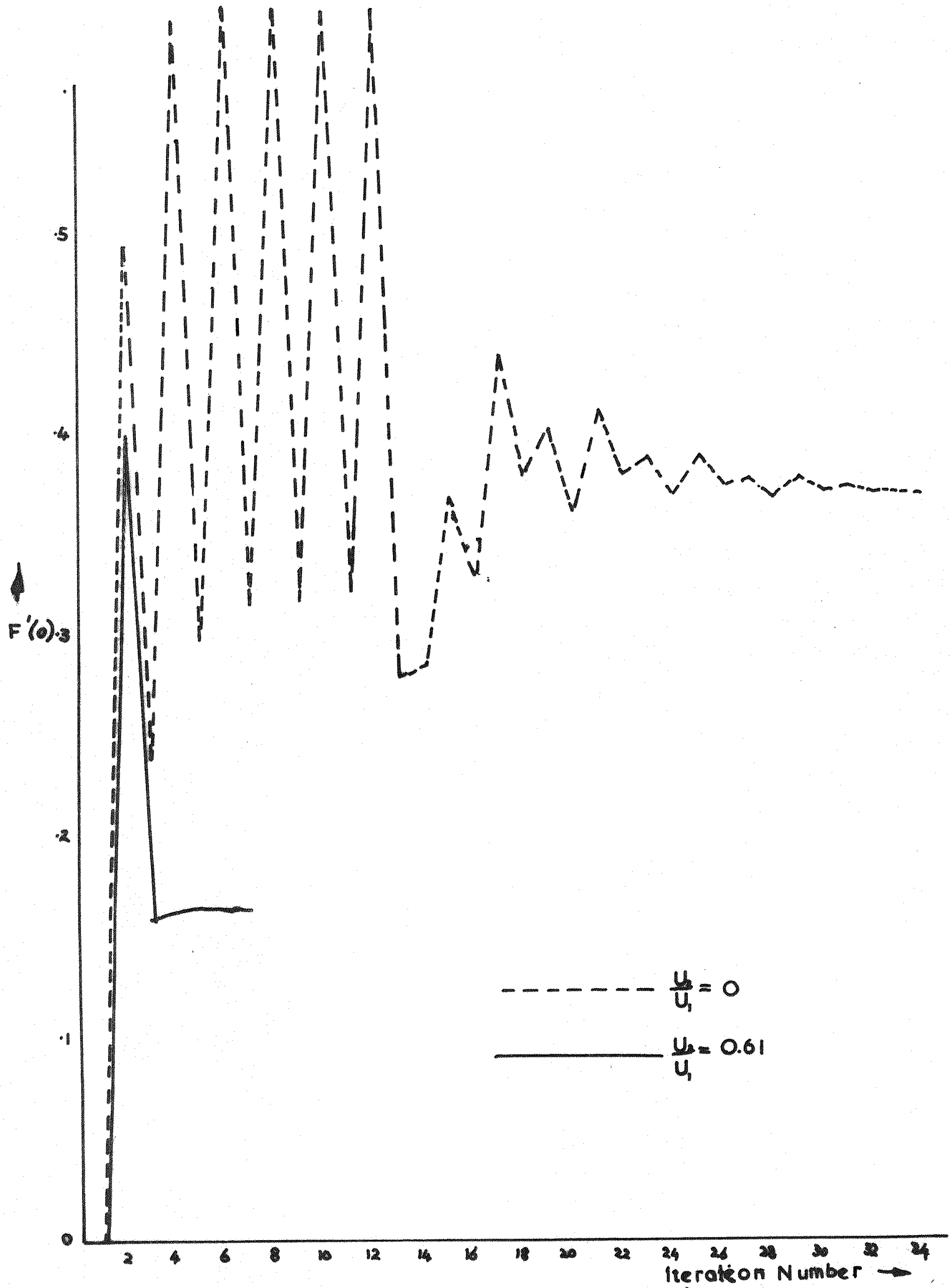

Convergence of Fil) for Difterent Velocity Ratios Fifure 4. 


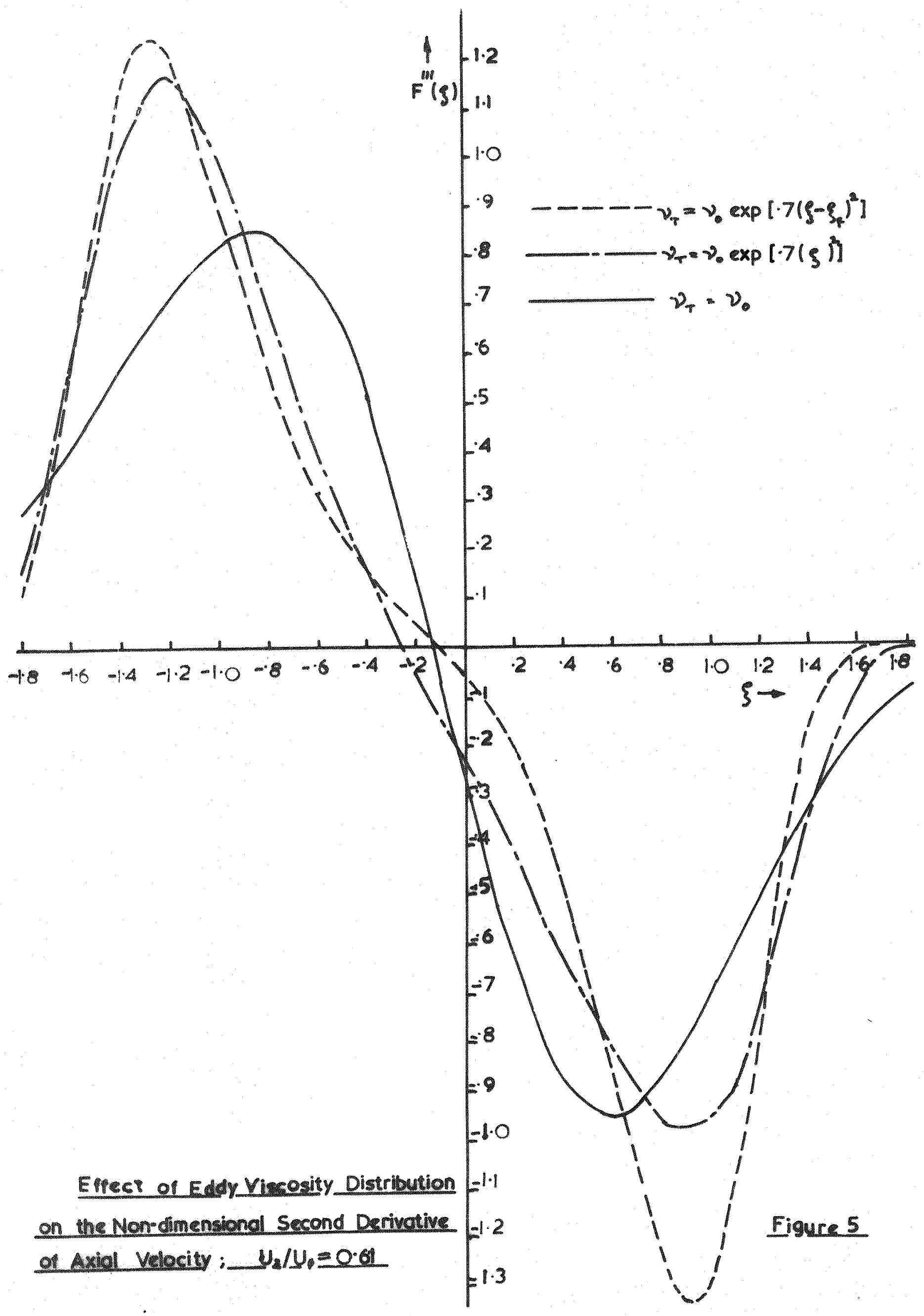




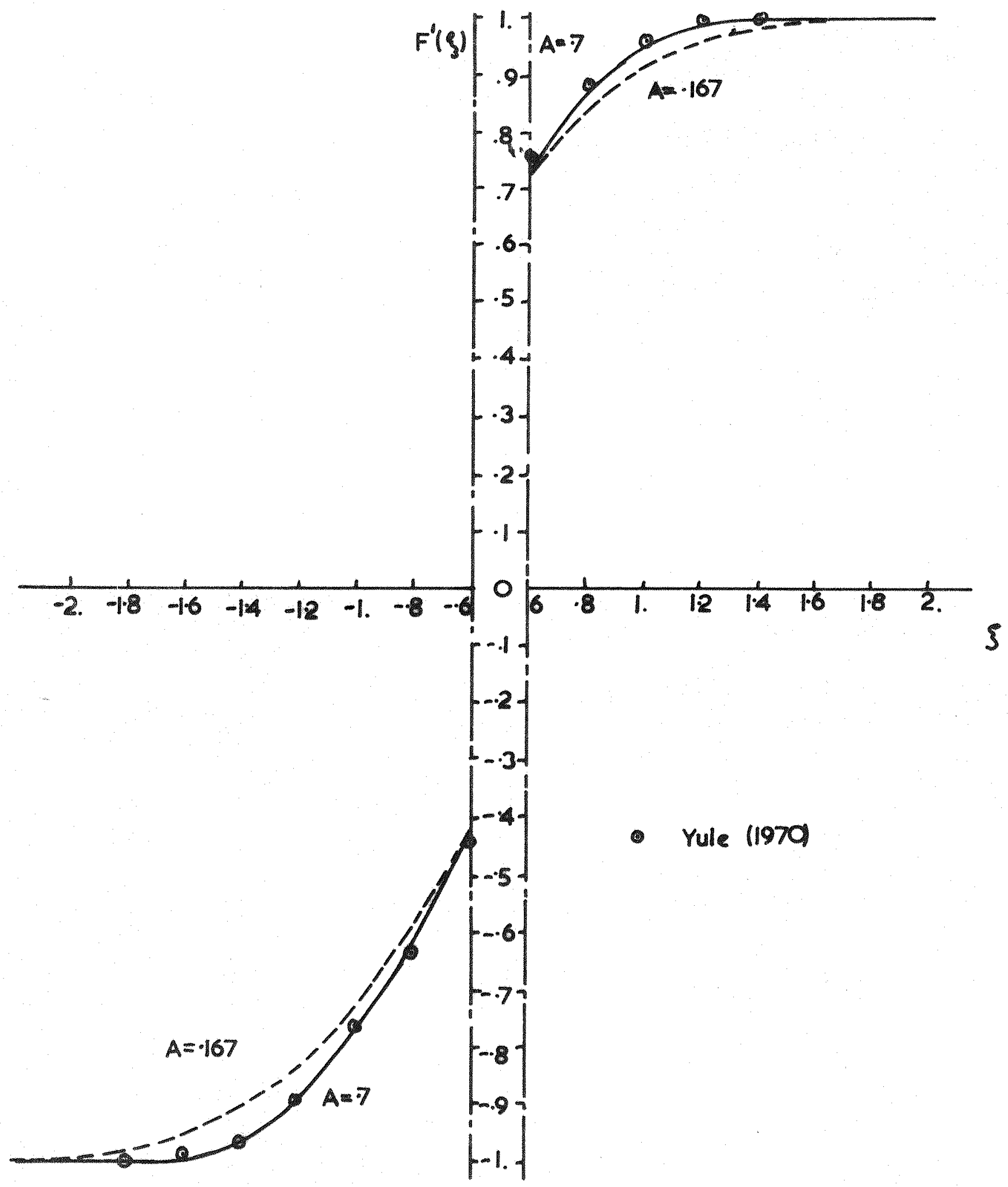

Effect of Choice of Constant A on Numerical Solution $\underline{U}_{2} / \underline{U}_{1}=0.61$

Eigure 6 


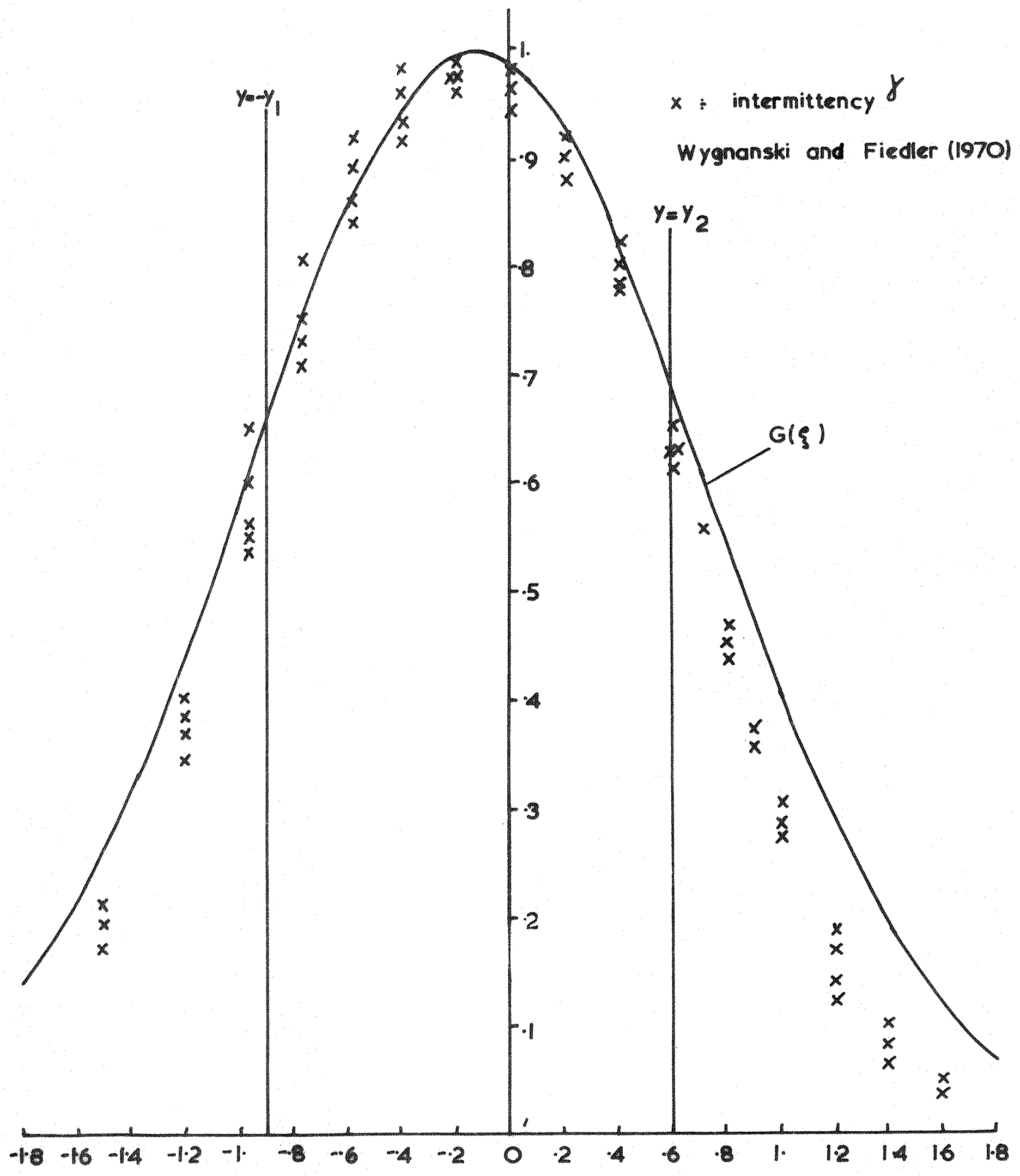

Comporison of the Function GISL with Experimental Measurements of

Wygaanaki and Fiedler

Eigure 7. 
- Experimental results Yule - - Exponential distribution of eddy viscosity, $A=07, j=13125, \sigma=.0033$.

- Constant eddy viscosity, $\sigma=0033$
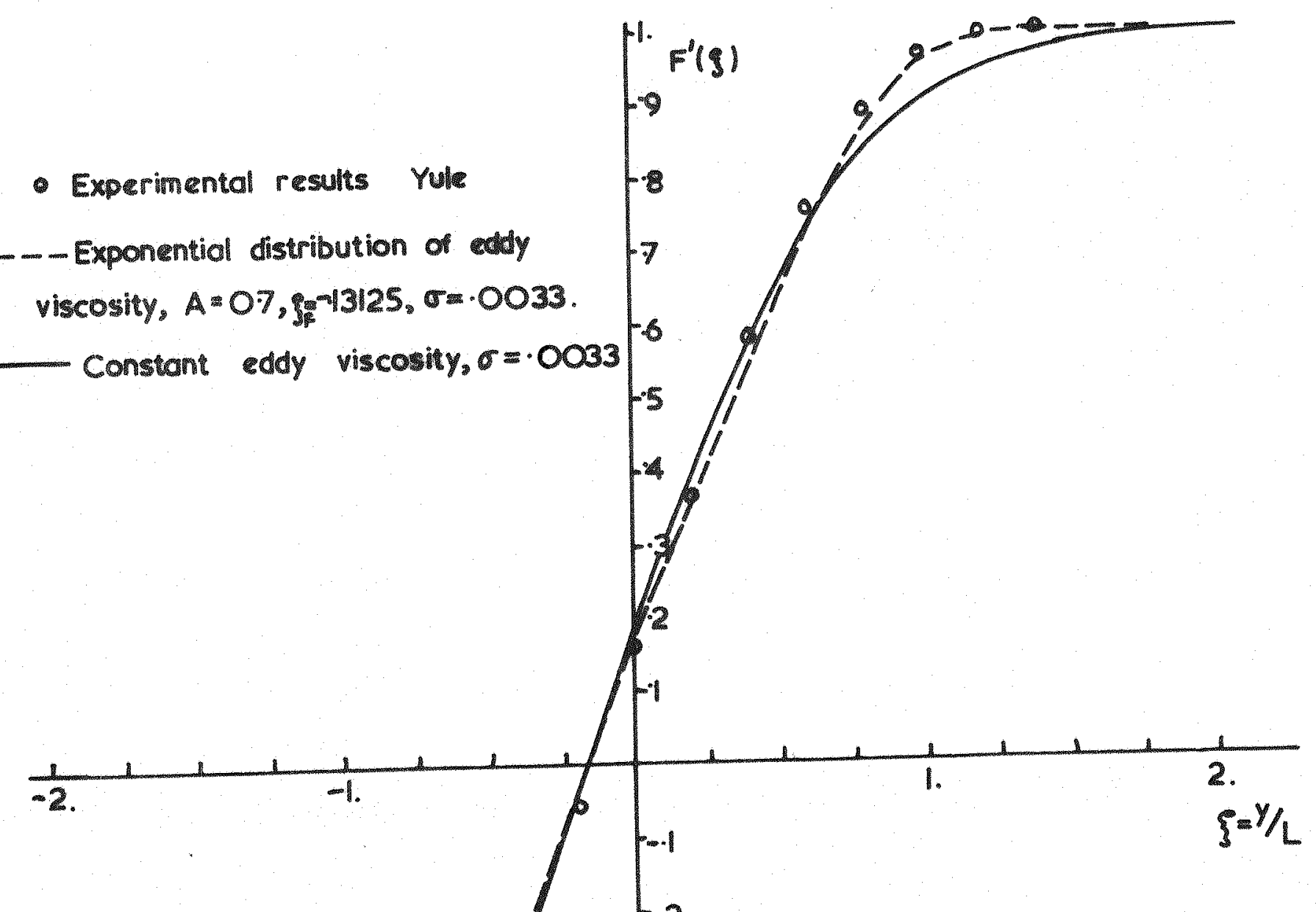

Non-dimensional Turbulent Velocity Profile $U_{2} / U_{1} \equiv .61$

Eigure 8 


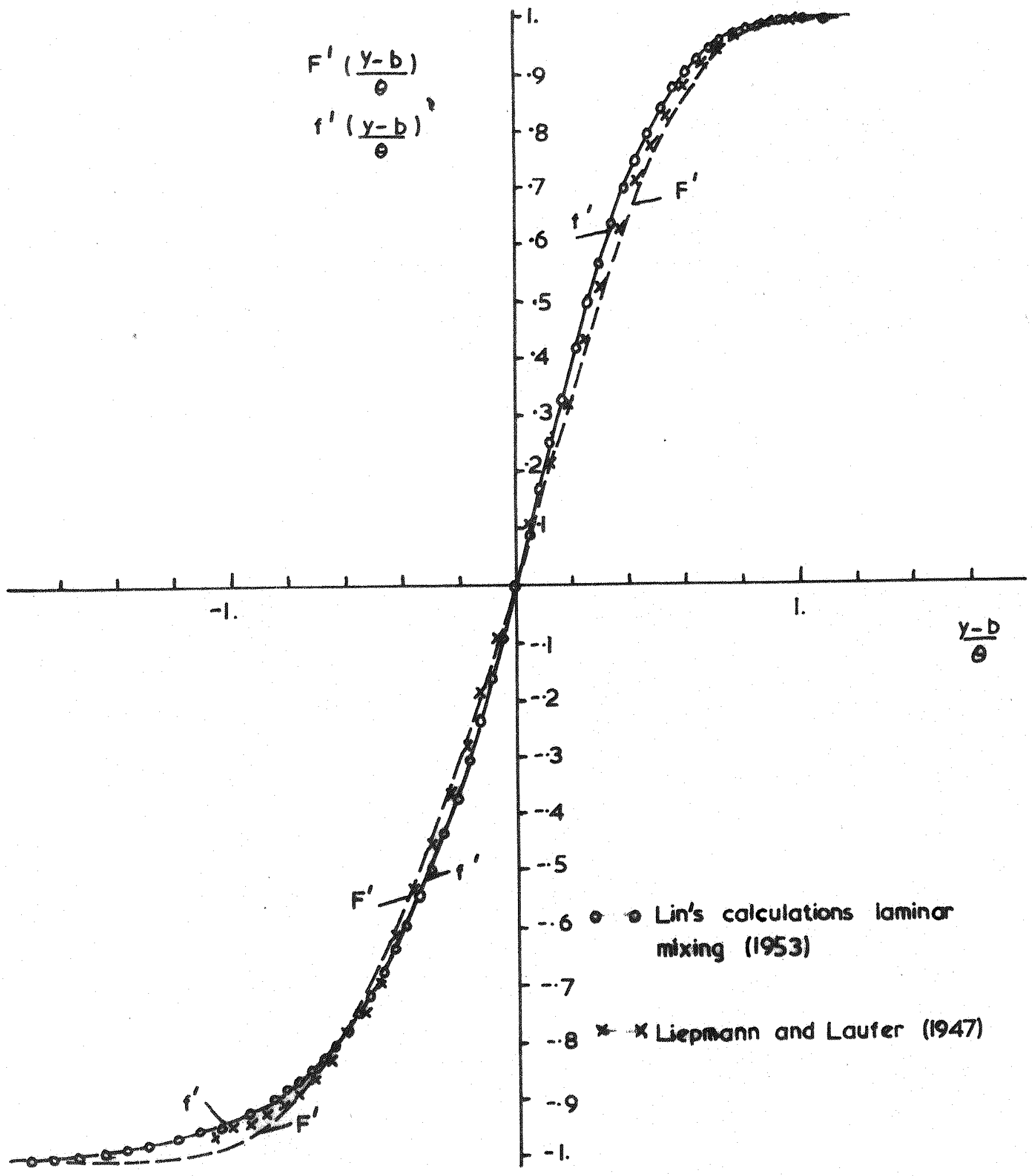

Non-dimensional Turbulent and Laminar Velocity Proflles, $\mathrm{U}_{2}=\mathrm{O}_{2}$ as Functions of $(y-b) / 0$. Figure 9 


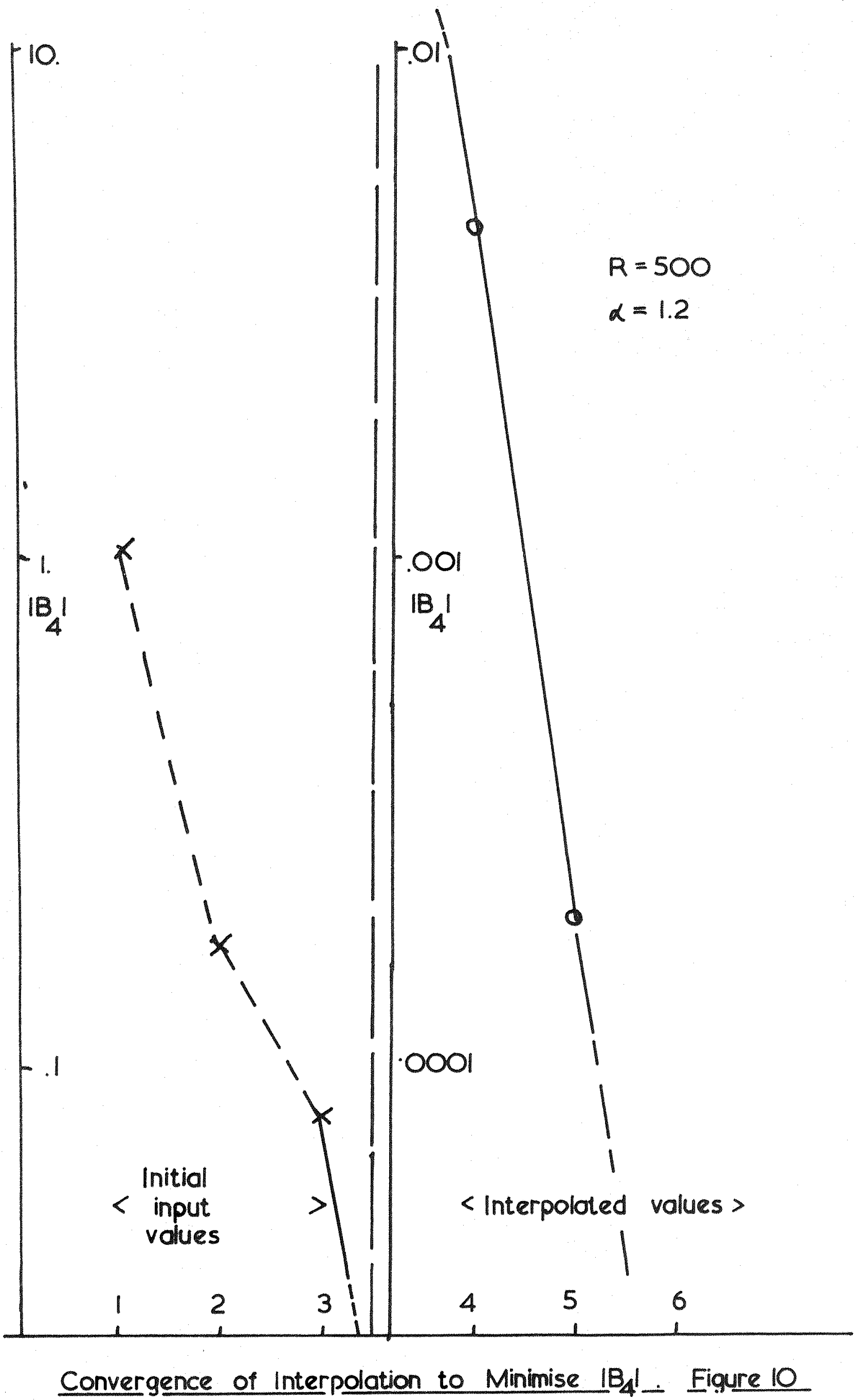




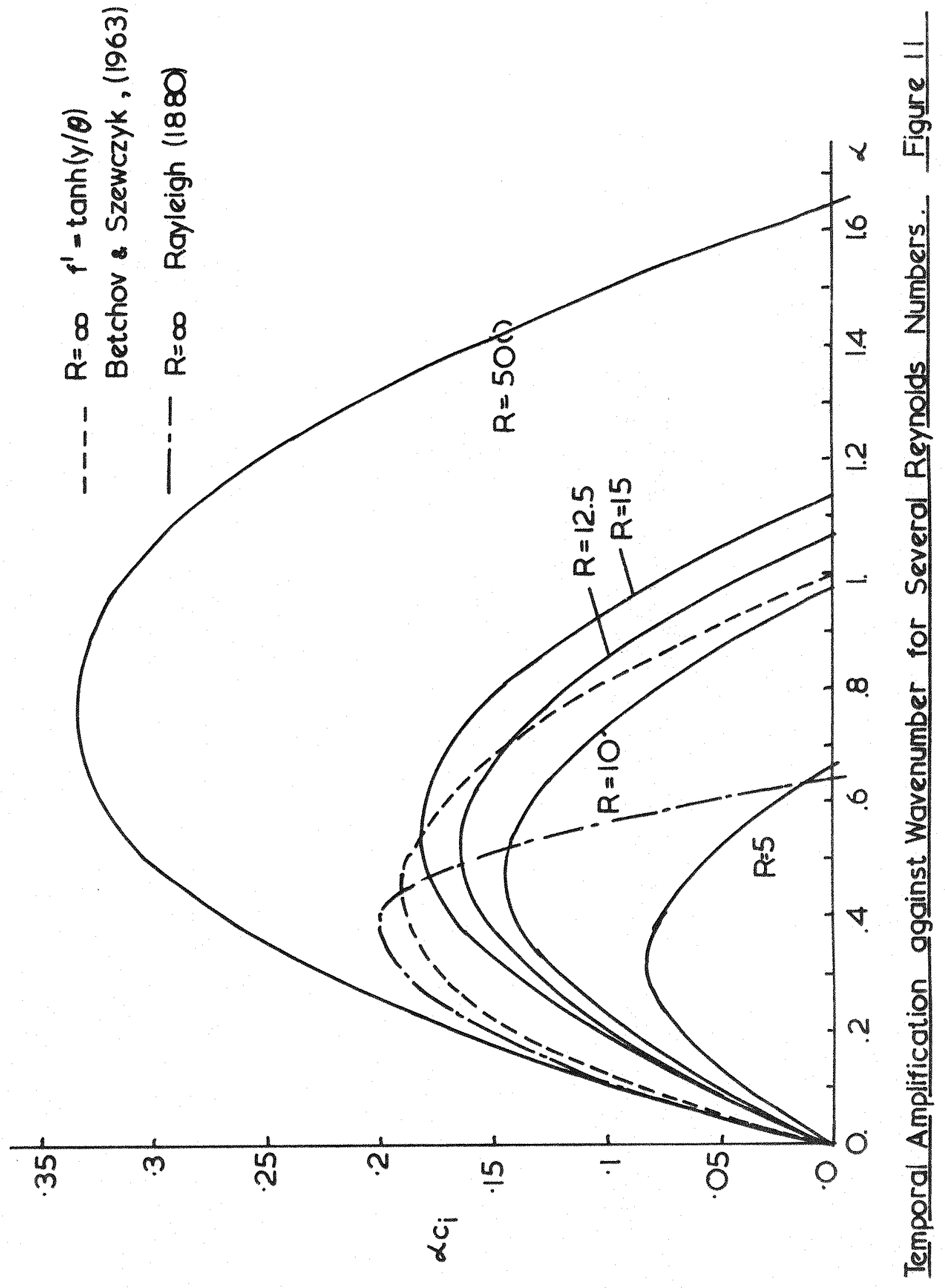




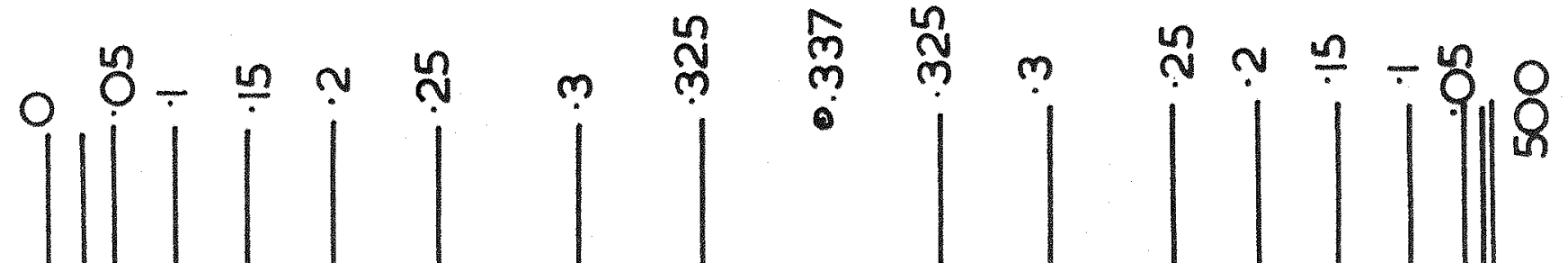

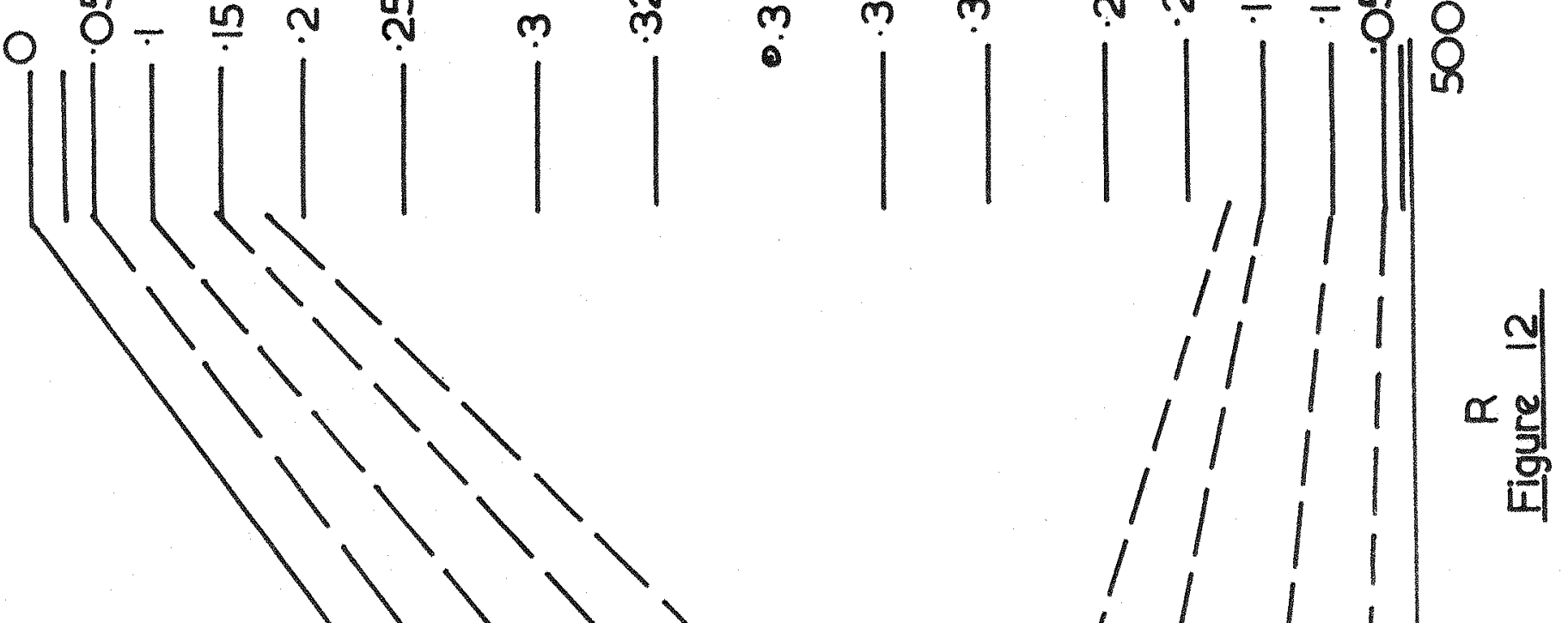




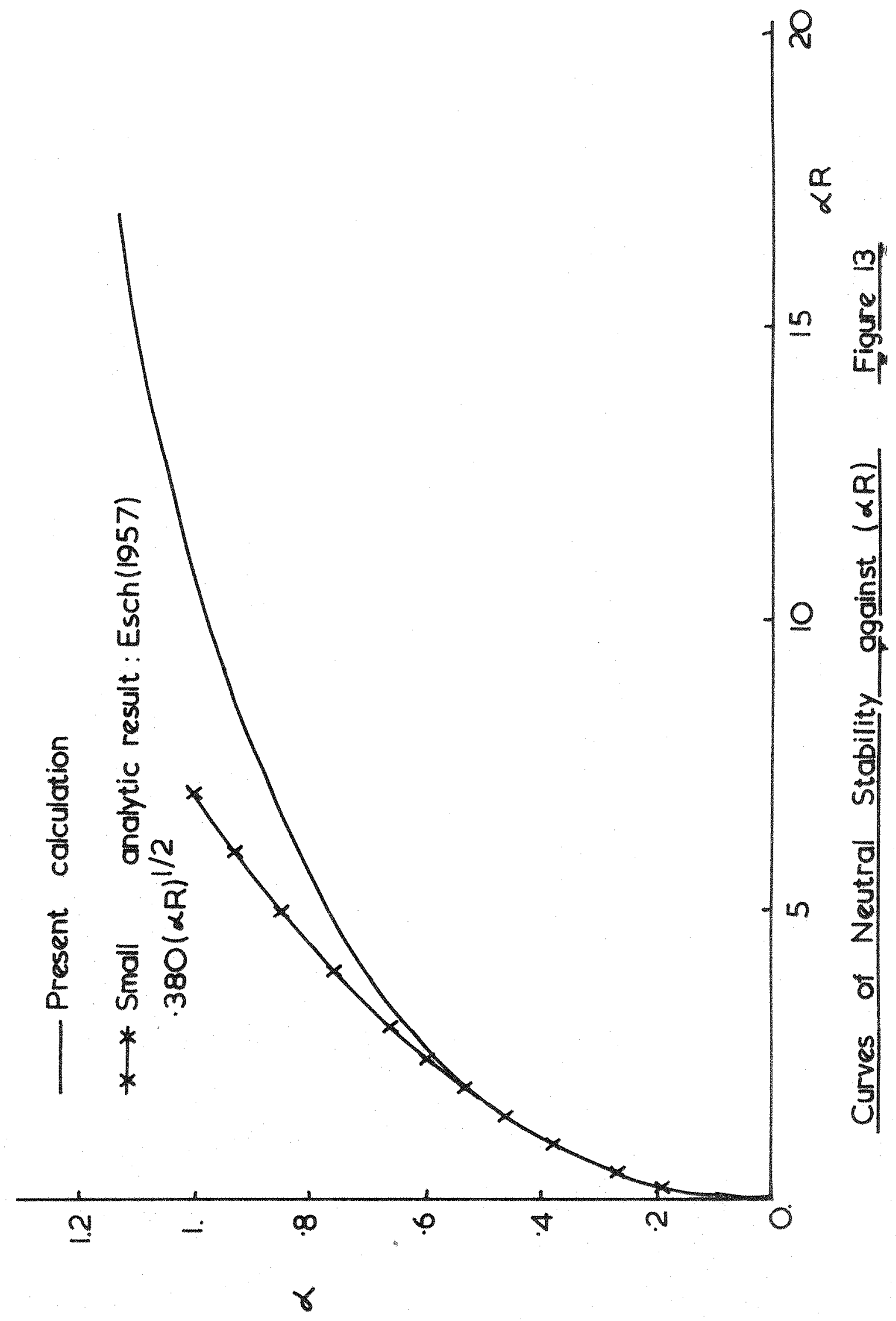




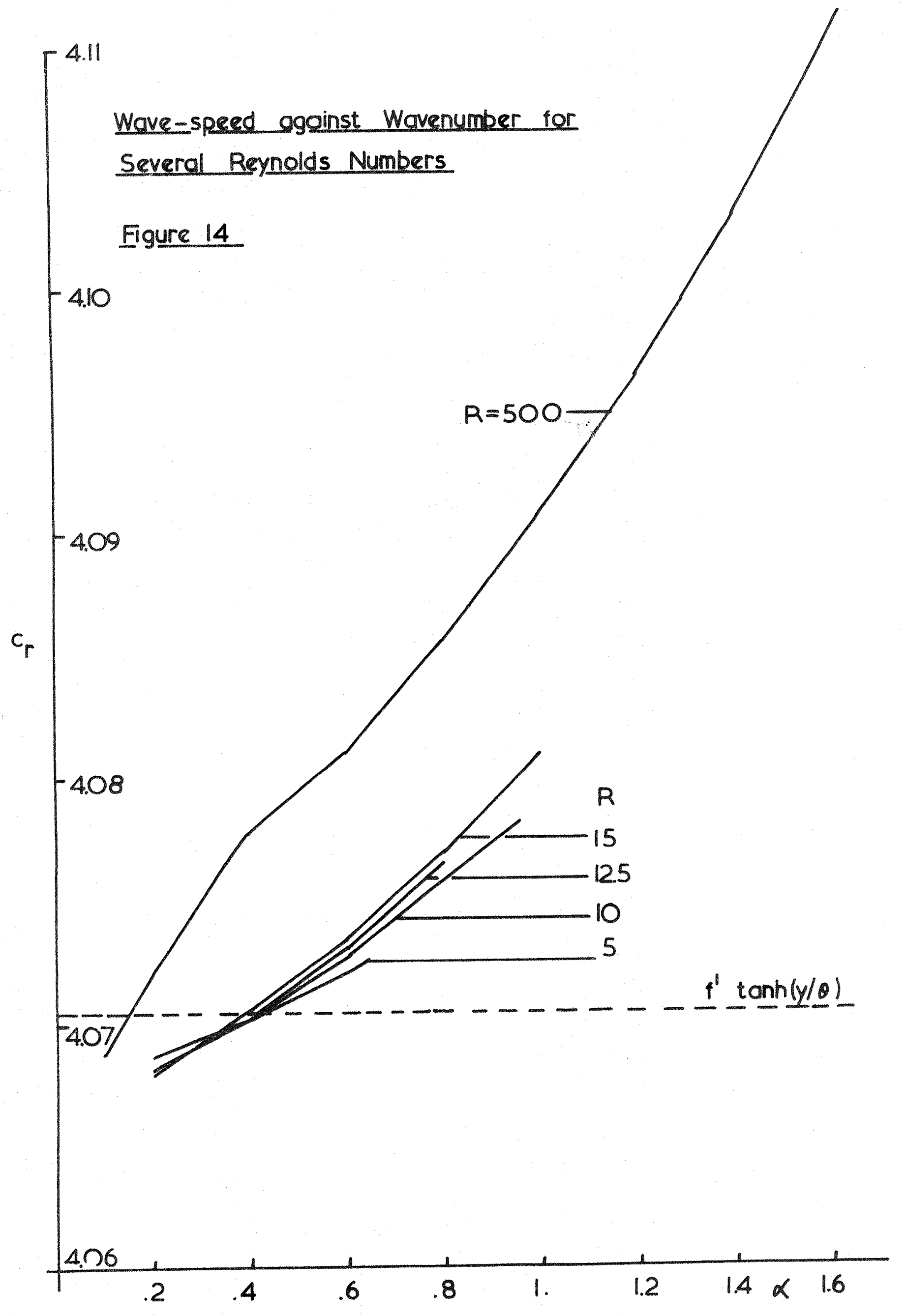




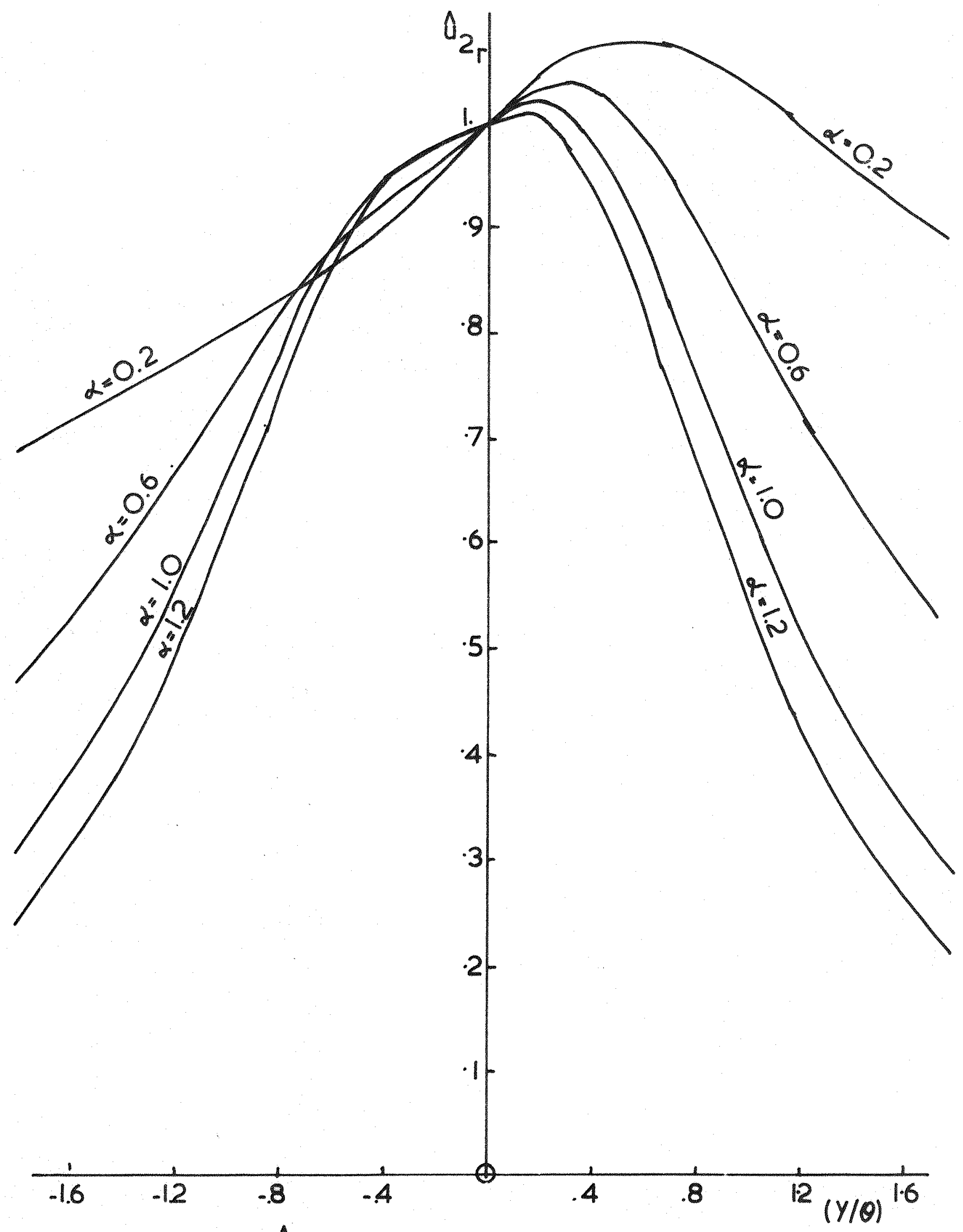

Eigenfunctions $\hat{u}_{2 r}(y / \theta)$ for Temporal Case and Several 


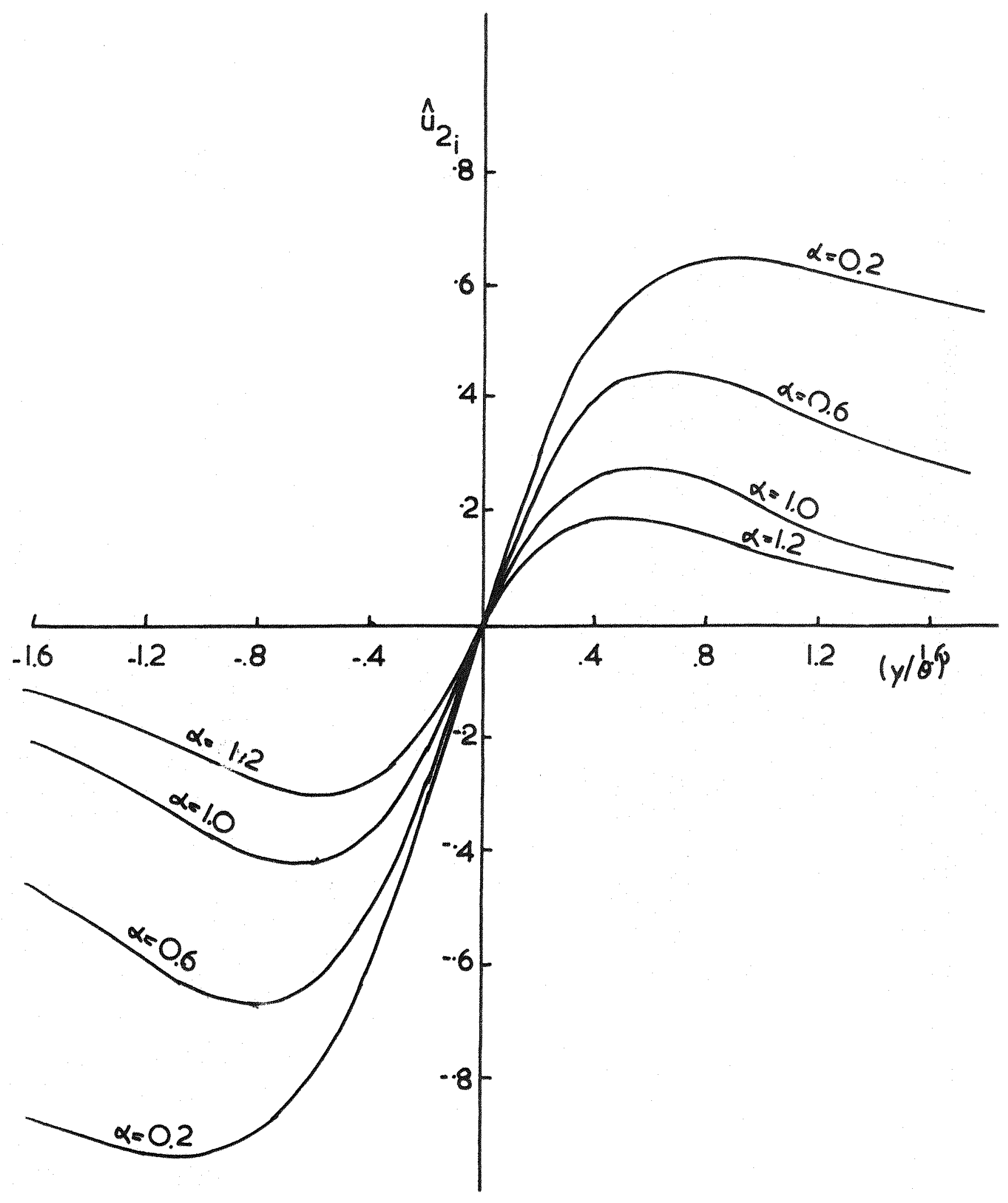

Eigenfunctions $\hat{U}_{2 i}(y / \theta)$ for Temporal Case and Several Wavenumbers Figure $15(b)$ 


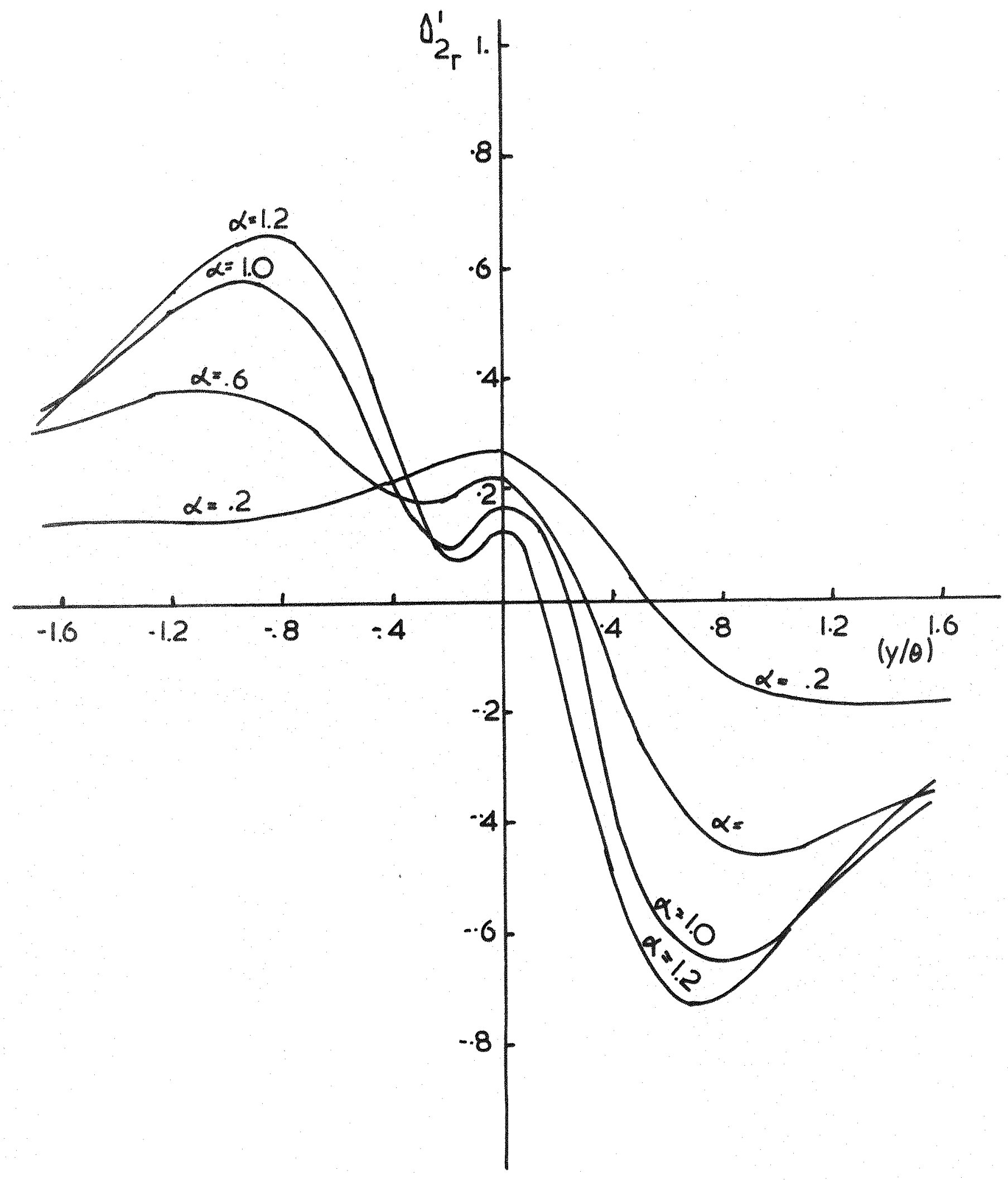

Derivatives $\hat{u}_{2 r}^{\prime}(y / \theta)$ of Eigenfunctions for Temporal Case and Several Wavenumbers Figure 15(c) 


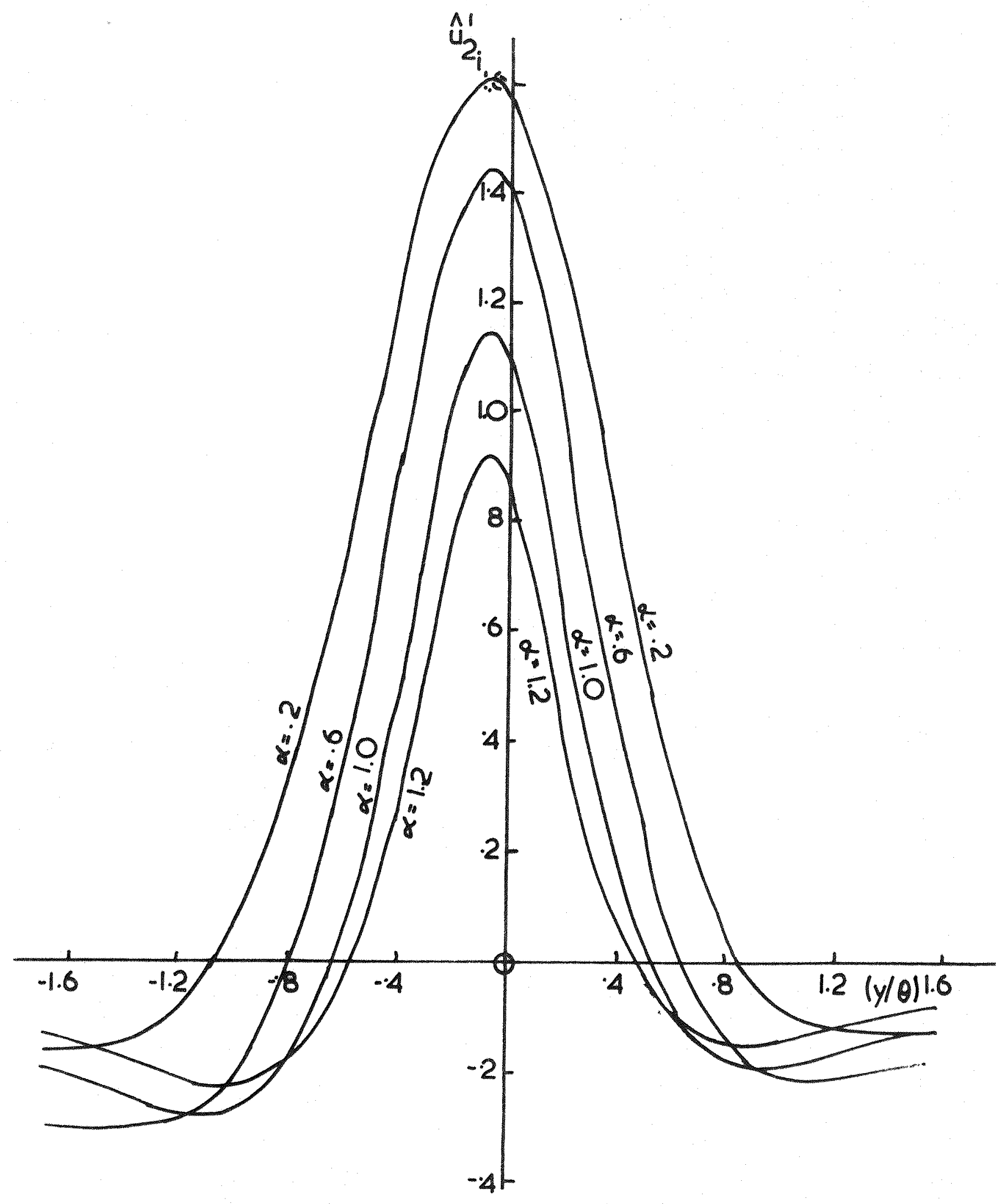

Derivatives $\hat{u}_{2_{i}}^{\prime}(y / \theta)$ of Eigenfunction for Temporal Case and Several Wavenumbers

Figure 15(d) 


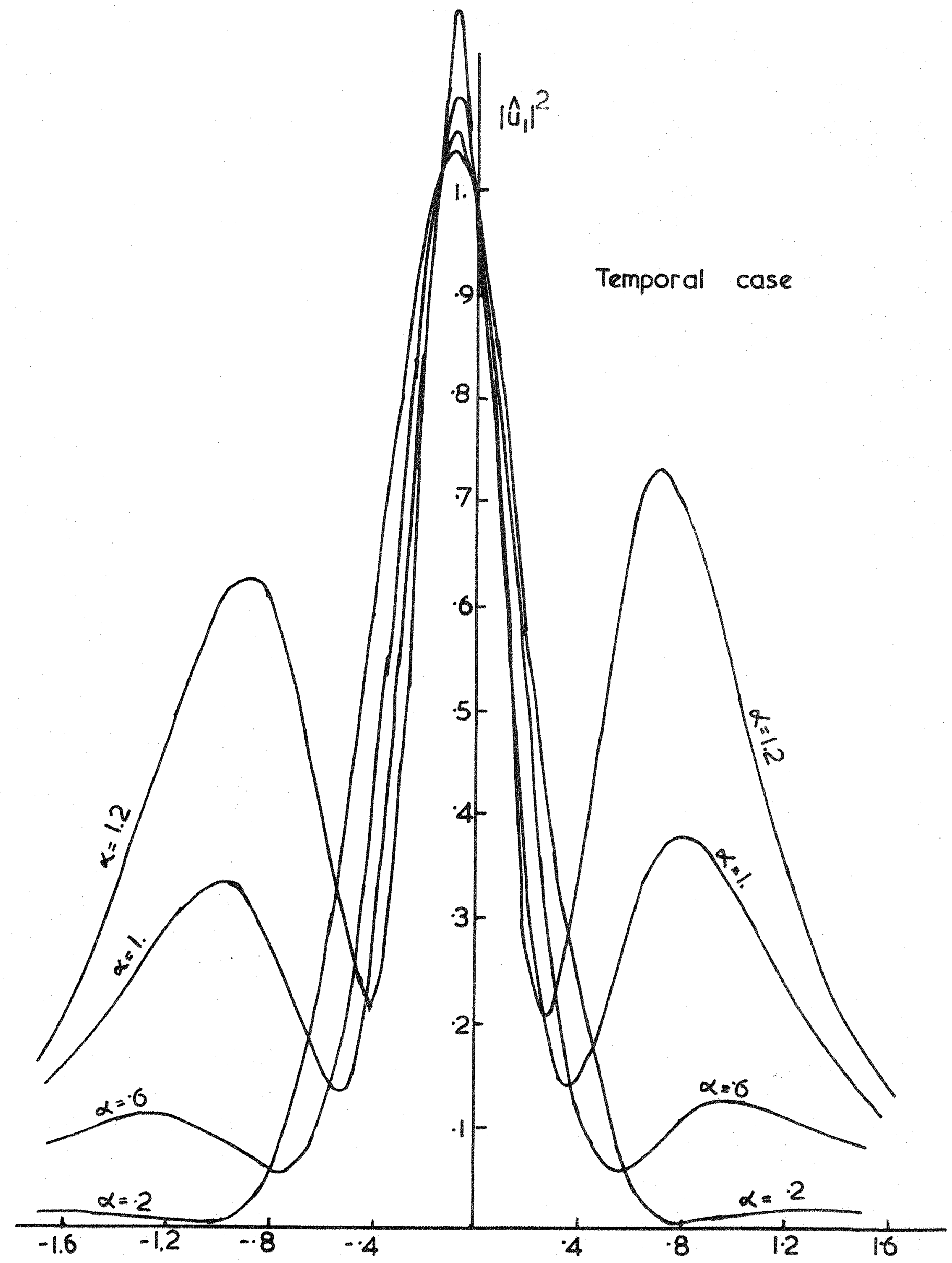

Distribution of $\left.\int \hat{u}_{1}\right|^{2}$ for Several Wavenumbers Figure 16 


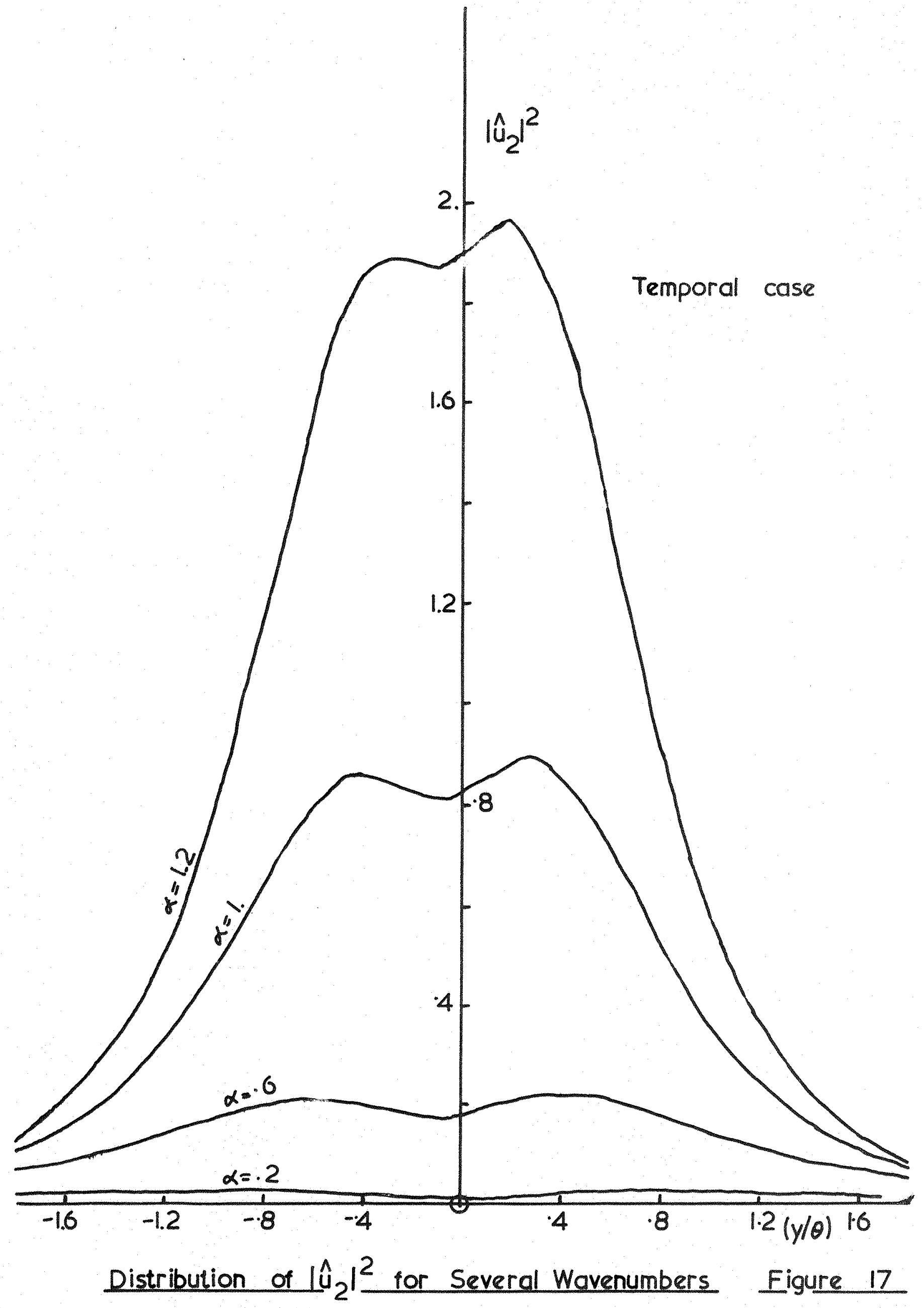




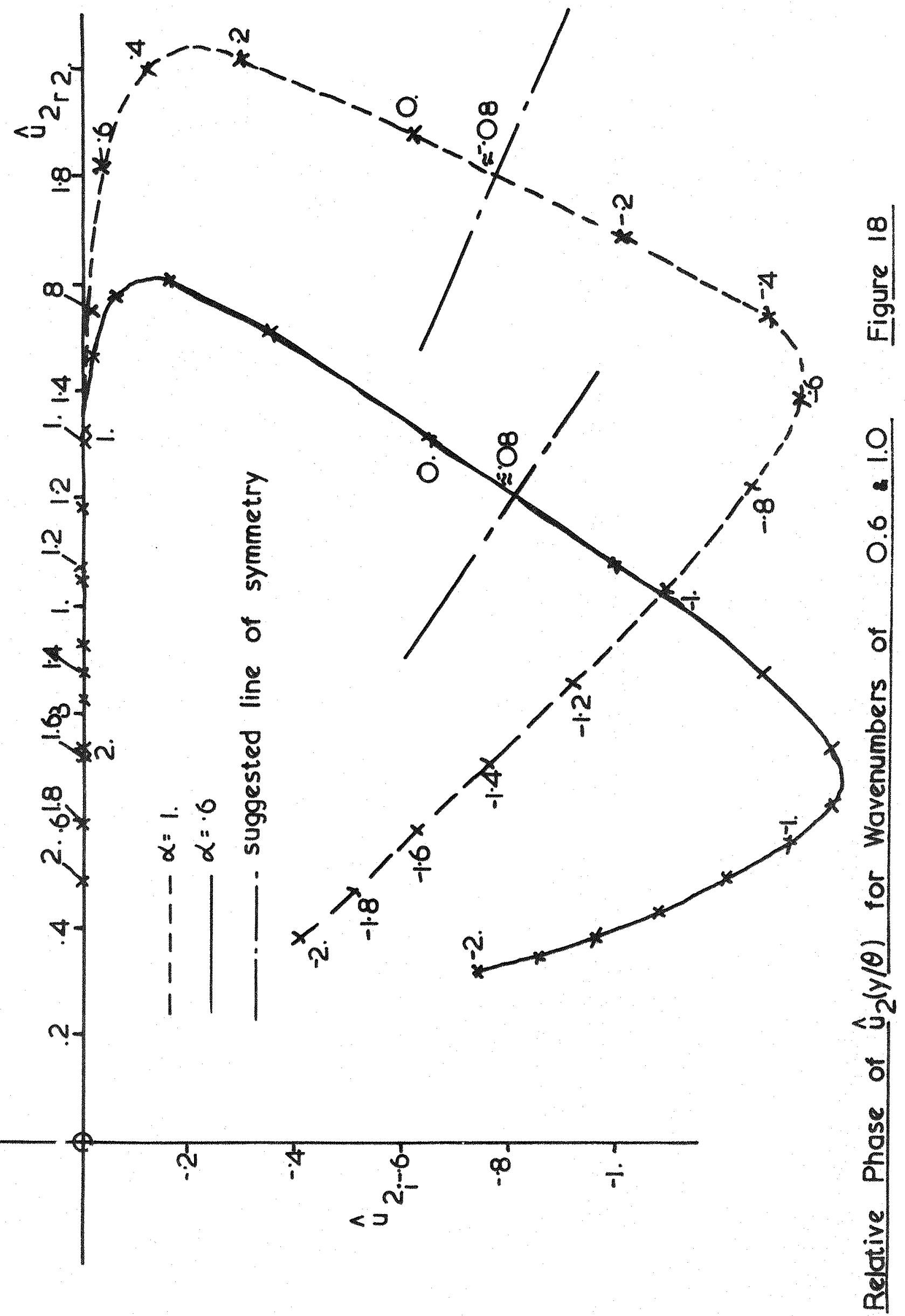




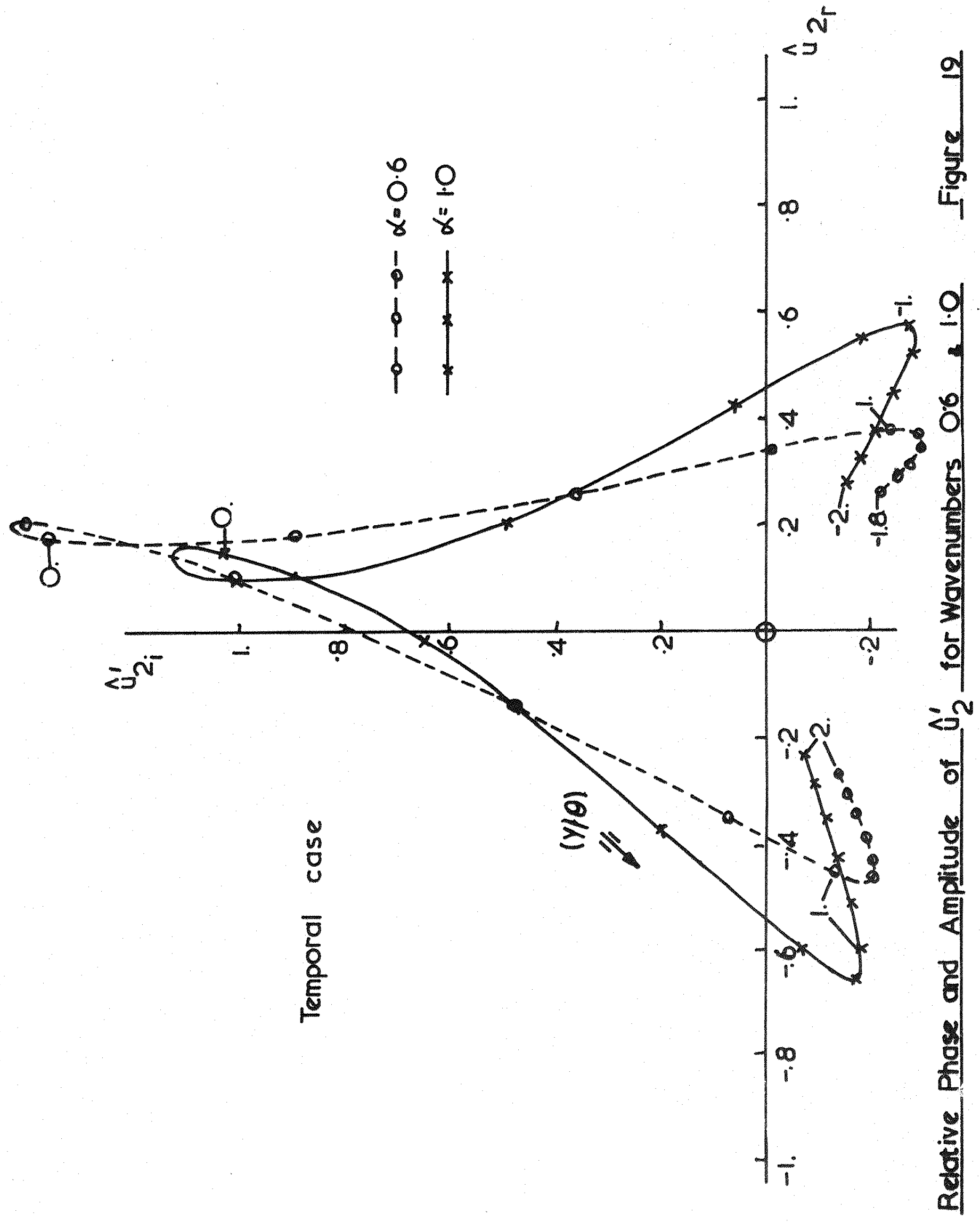




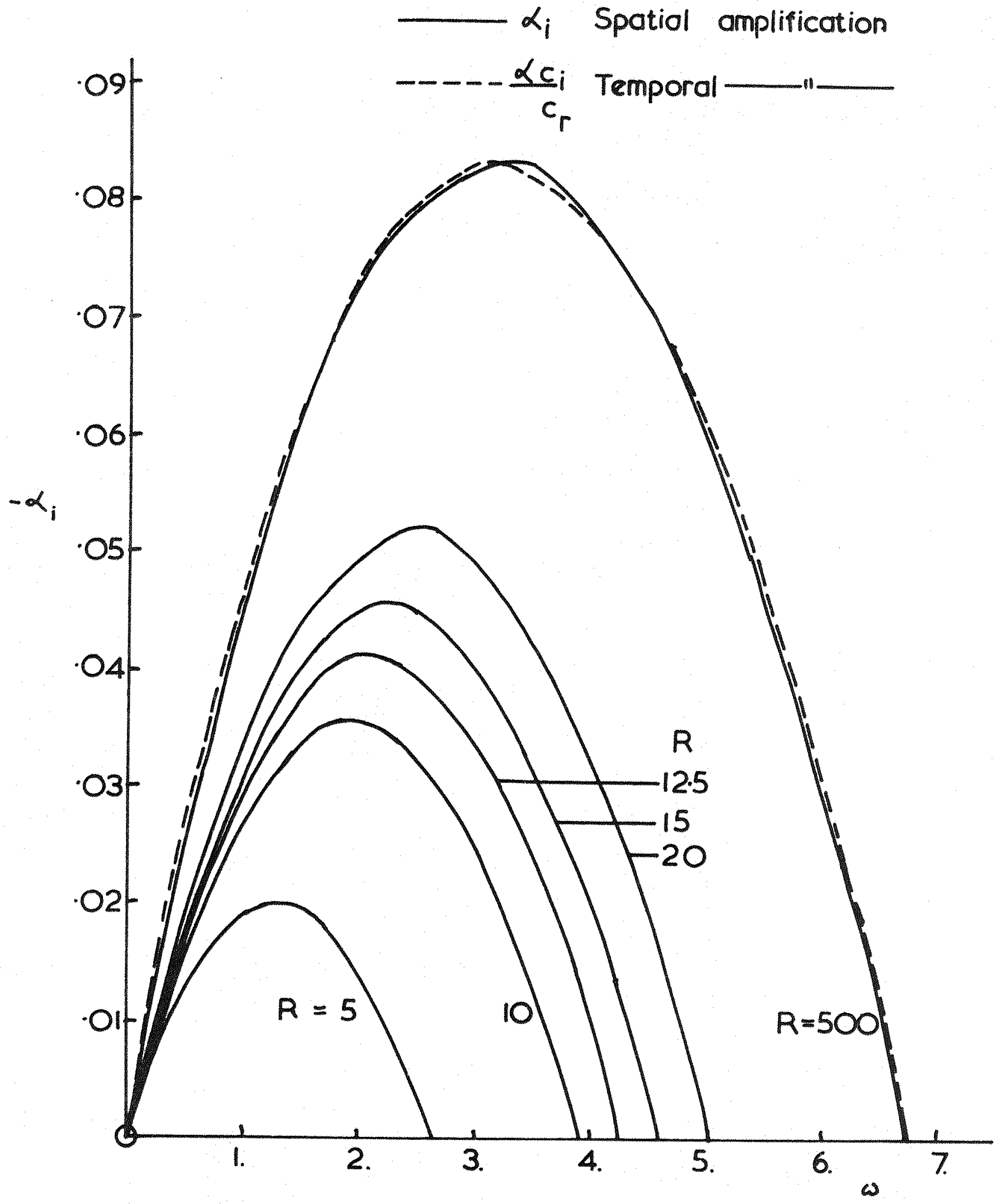

Spotial Amplification ggoinst Frequency for Several Reynolds Numbers Figure 20 


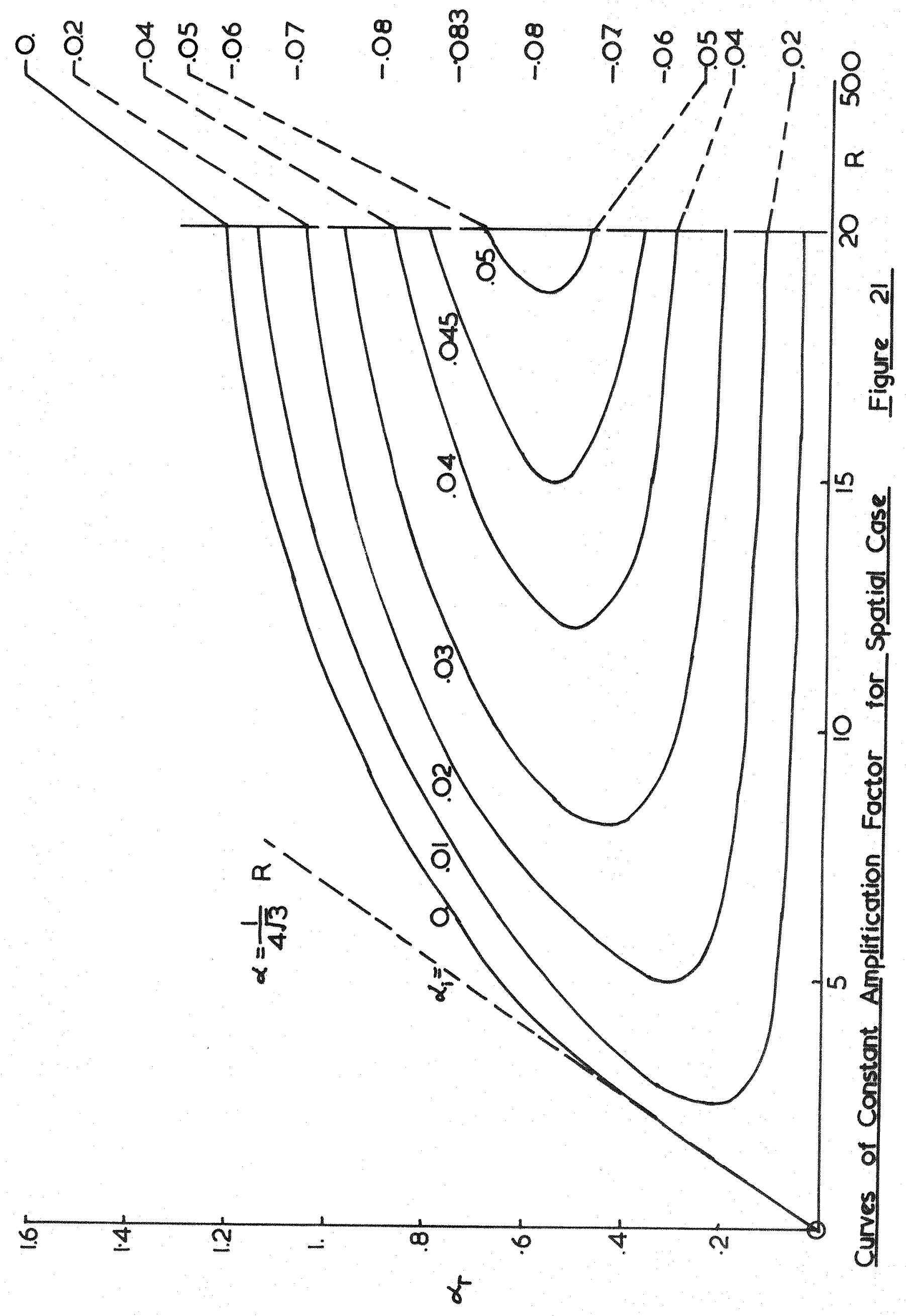




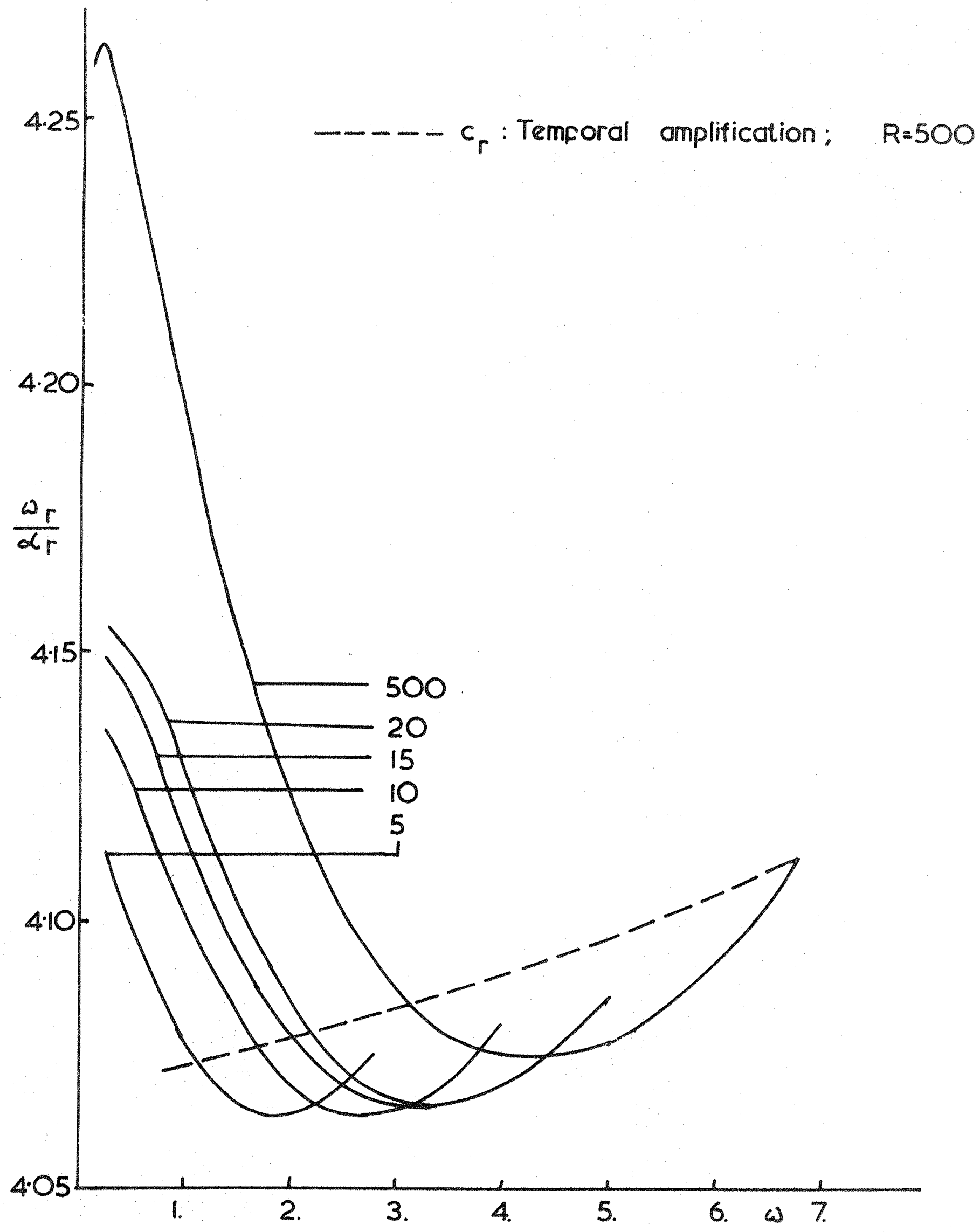

Phase Velocity against Frequency for Several

Reynolds Numbers Figure 22 


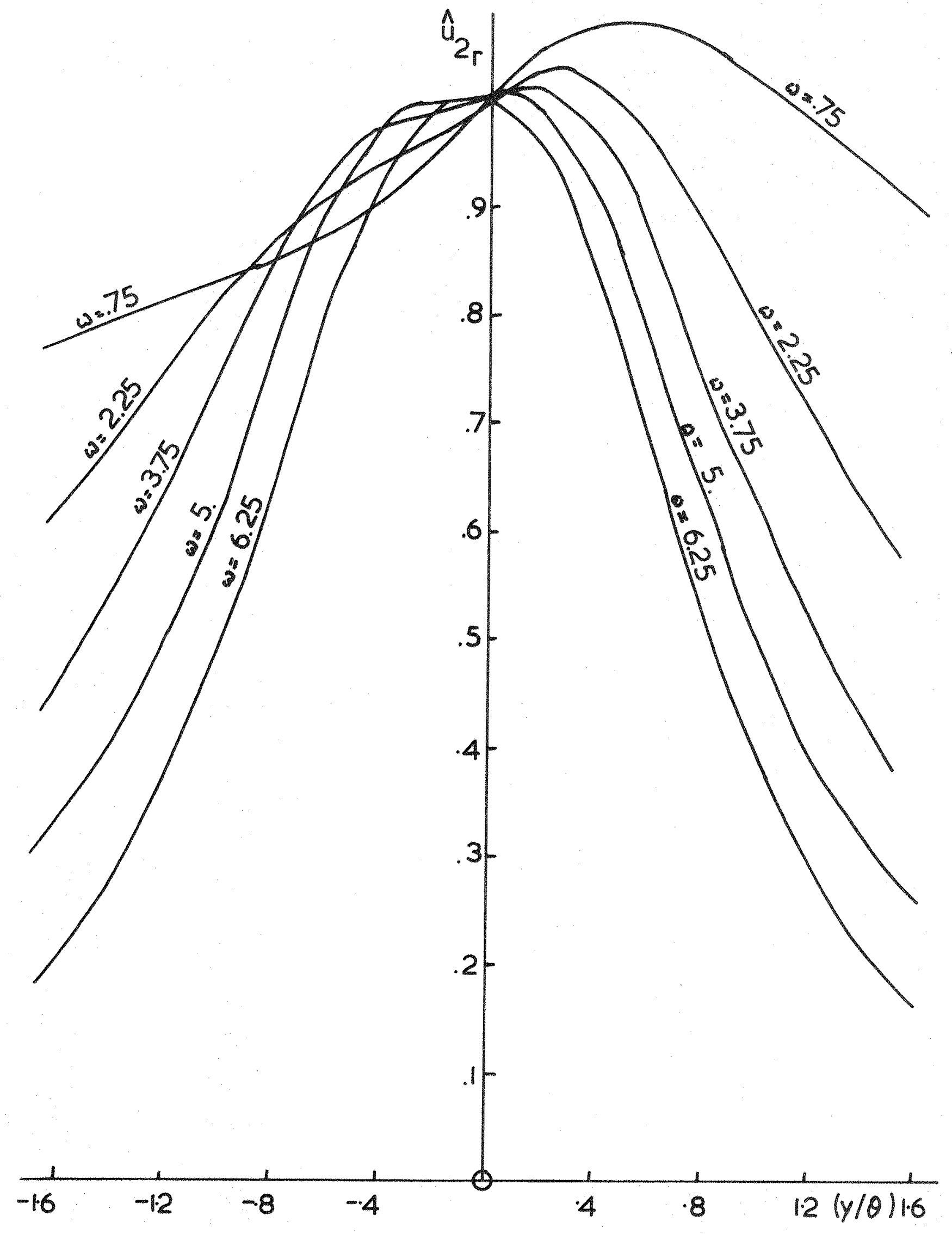

Eigenfunctions $\hat{u}_{2 r}(y / \theta)$ tor Spatial Case and Several

Frequencies

Figure 23(a) 


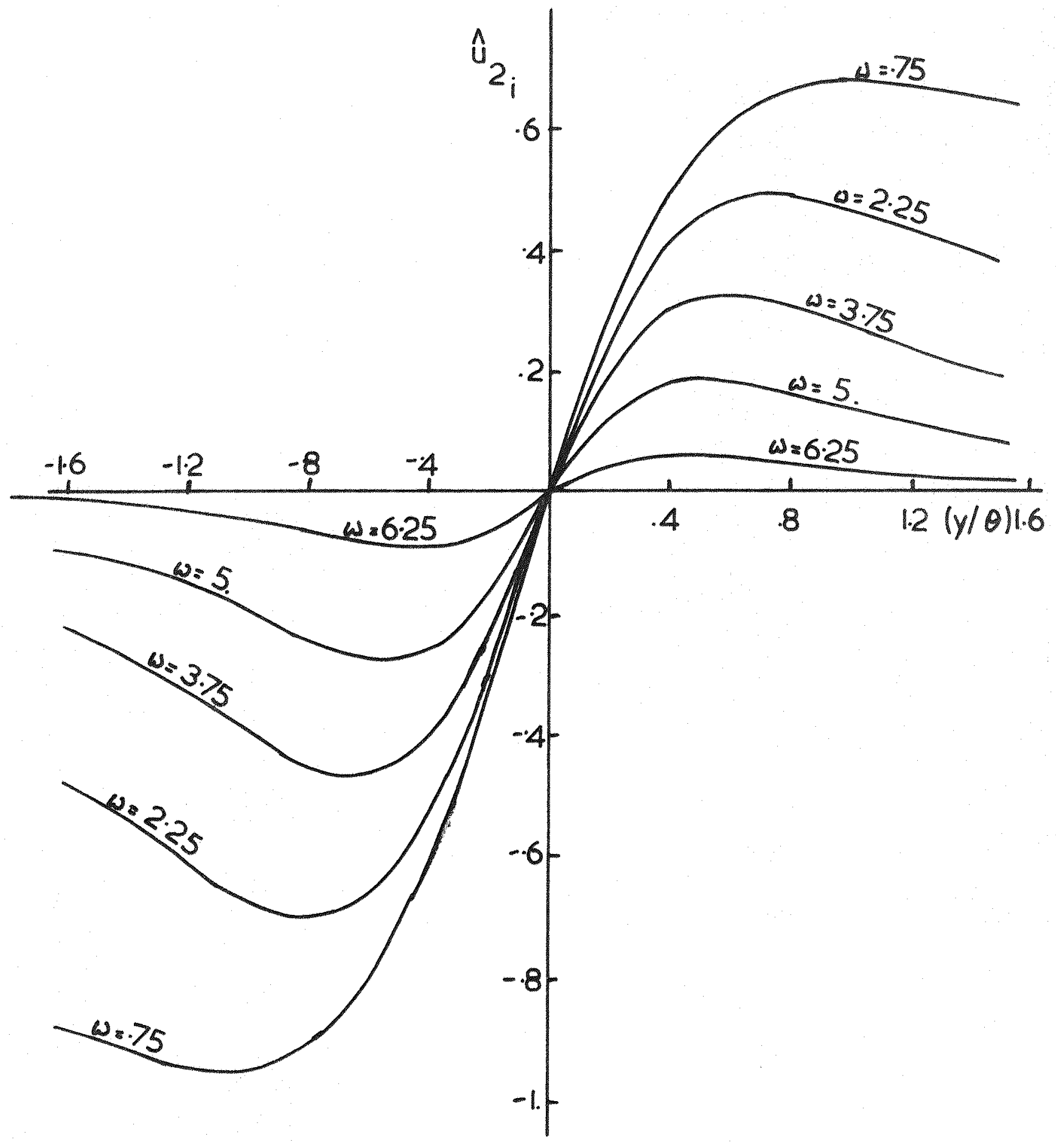

Eigenfunctions $\hat{u}_{2}(y / \theta)$ for Spatial Case and Several

Frequencies

Figure $23(b)$ 


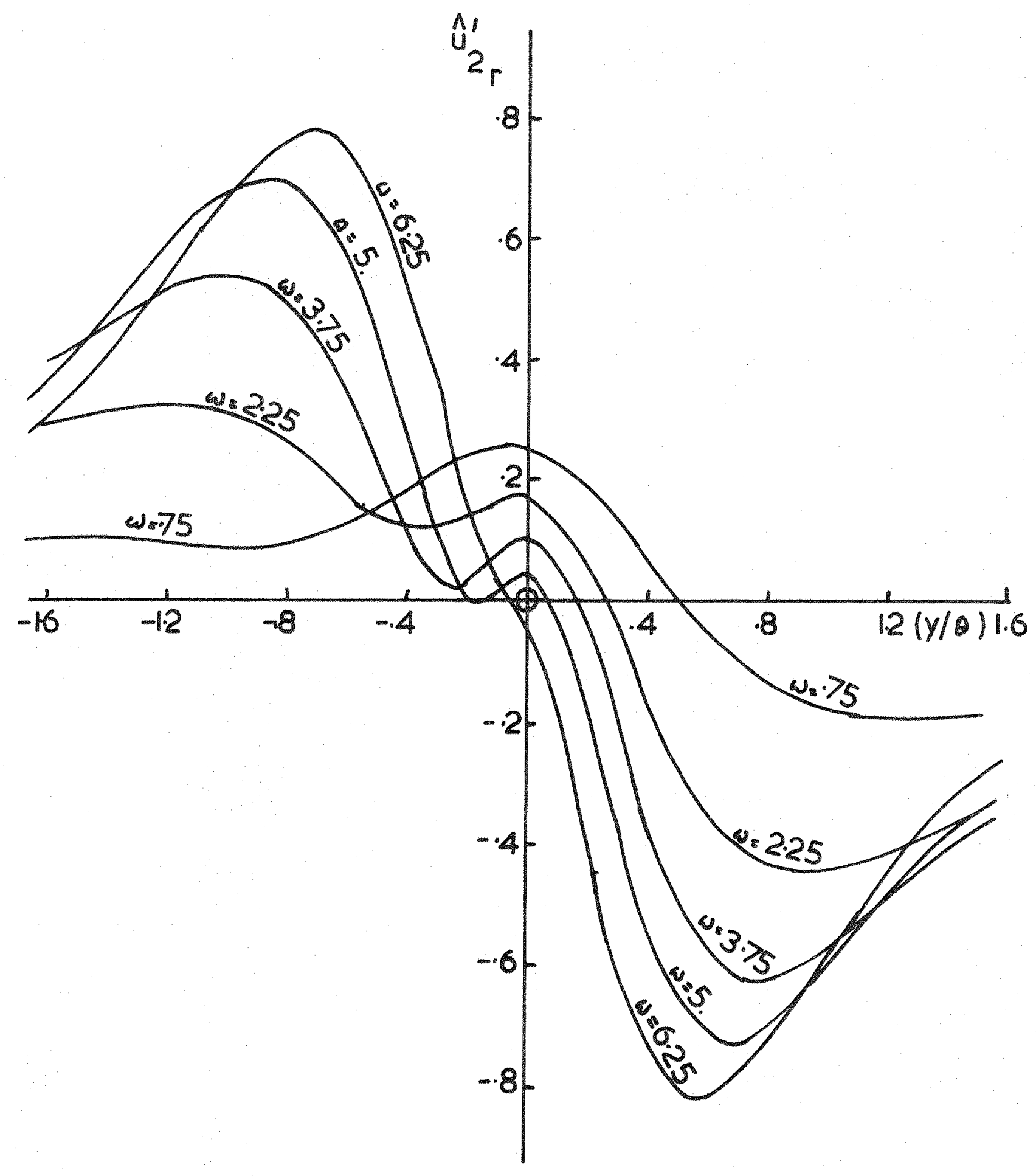

Derivatives $\hat{U}_{2}^{\prime}(y / \theta)$ for Spatial case and Several

Frequencies

Figure 23(c) 


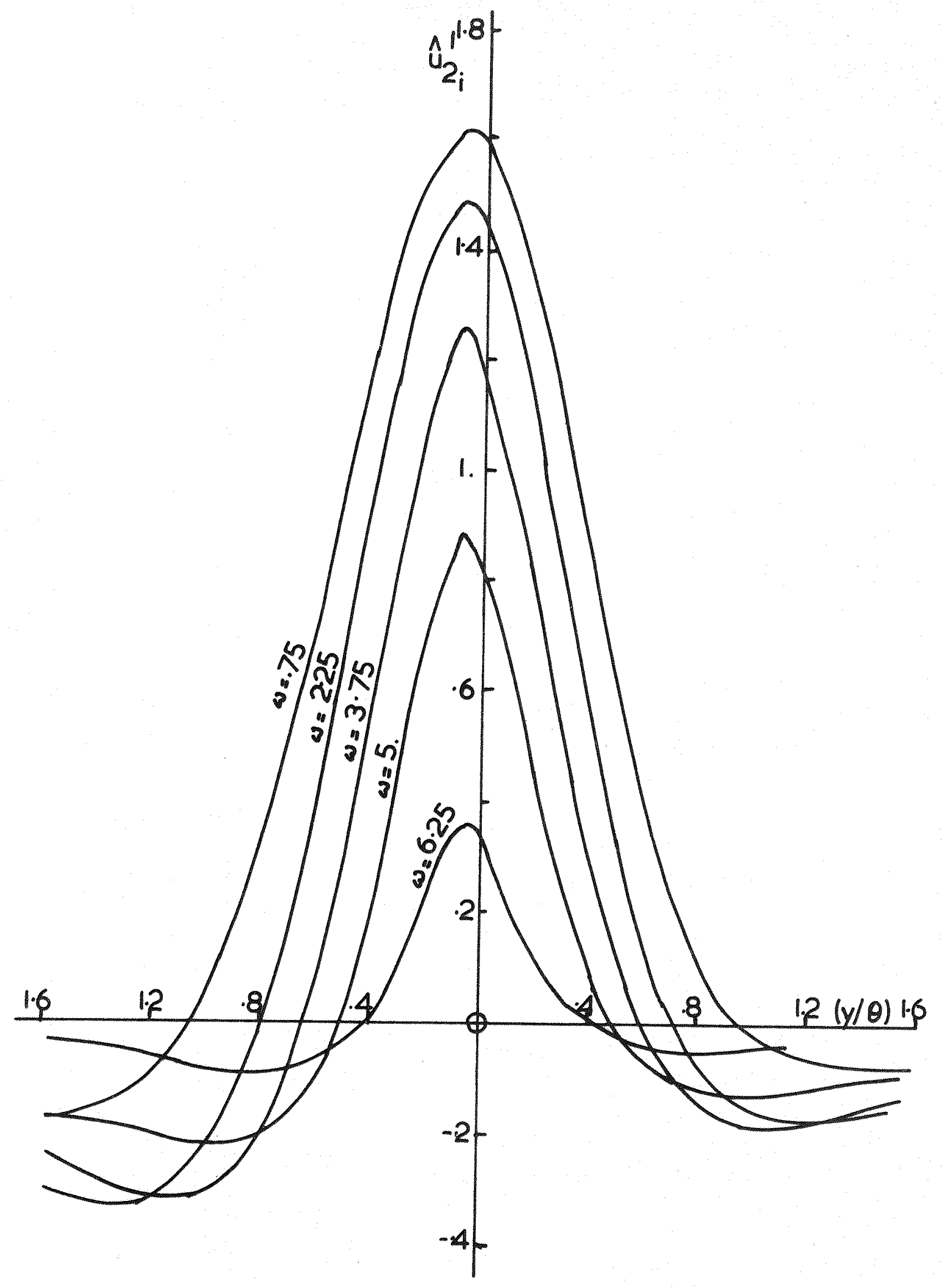

Derivatives $\hat{u}_{2}^{\prime}(y / \theta)$ for Spatial Case and Several

Frequencies

Figure 23(d) 


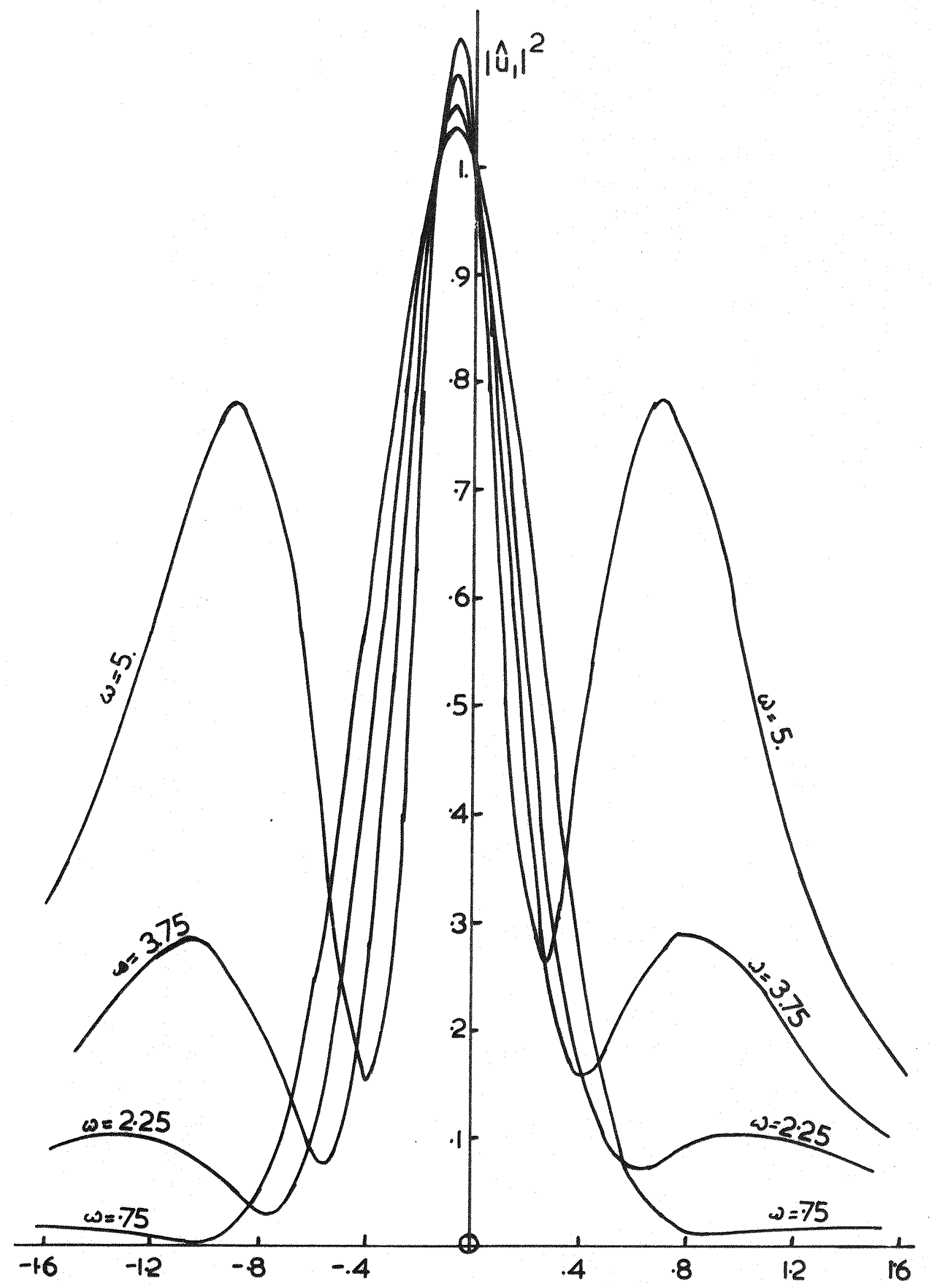

Distribution of $\left|\hat{u}_{1}\right|^{2}$ for Several Frequencies Figure 24 


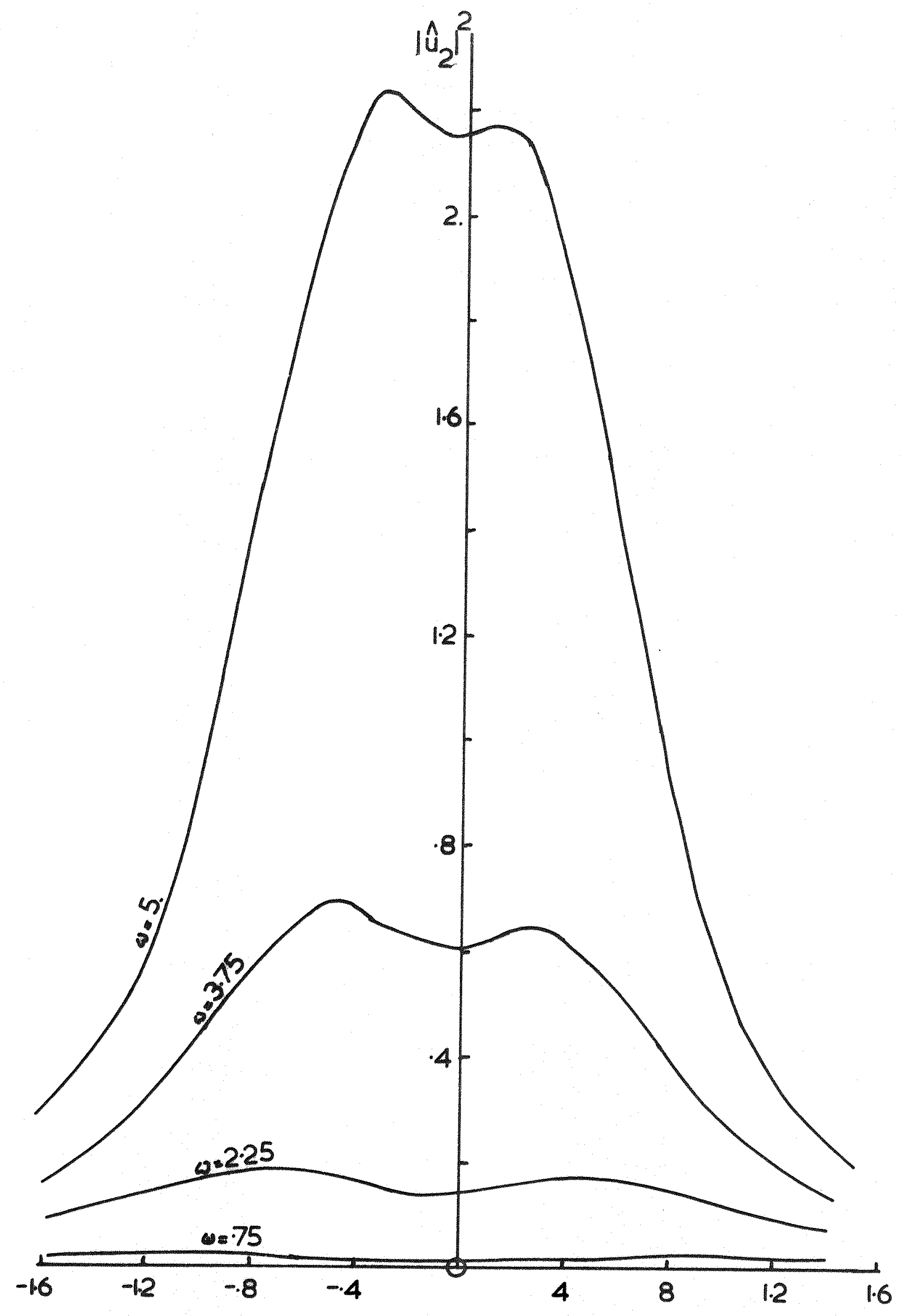

Distribution of $\left|\hat{u}_{2}\right|^{2}$ for Several Frequencies Figure 25 


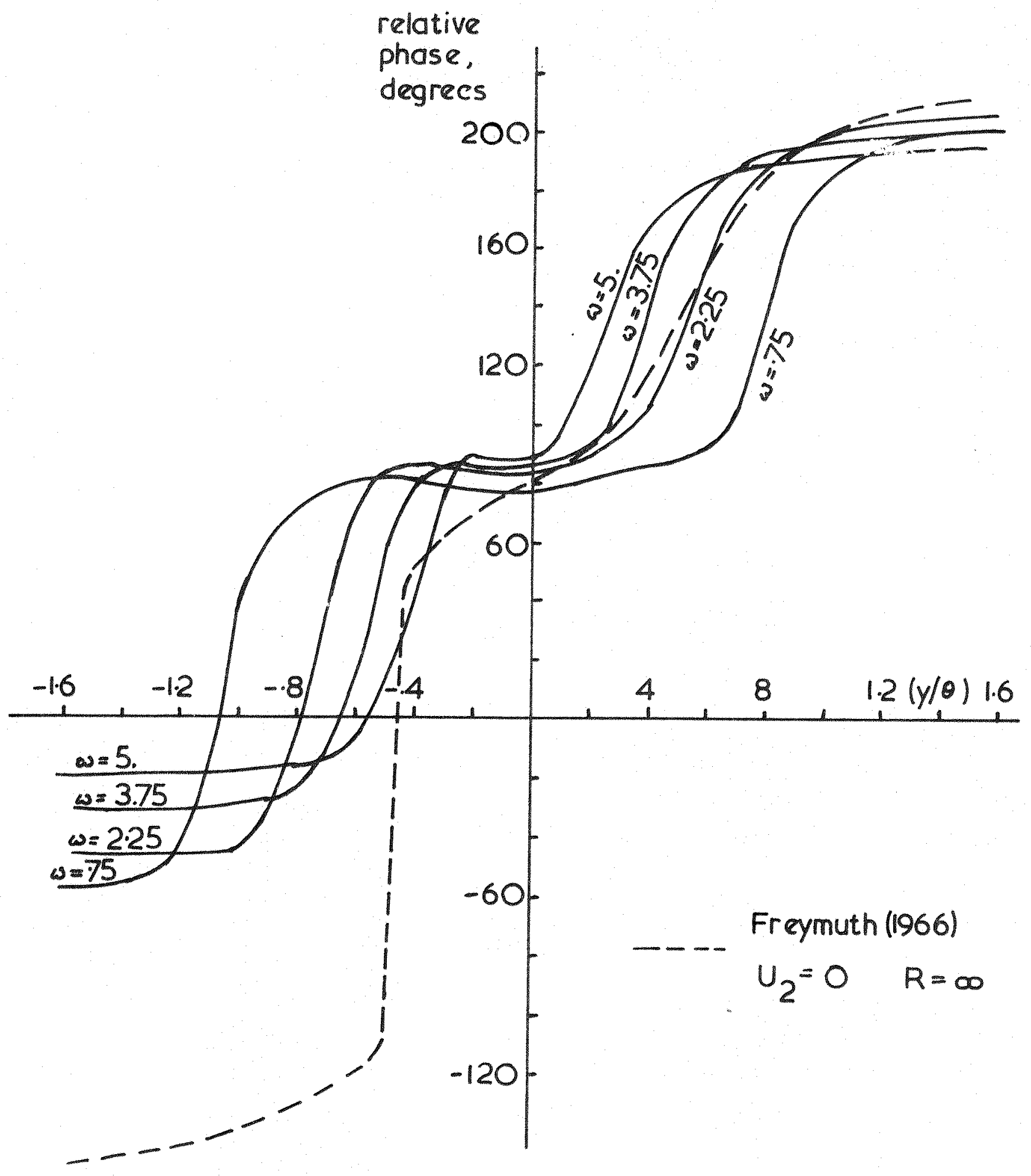

Relative Phase of $\hat{U}_{1}$ for Several Frequencies

Figure 26 


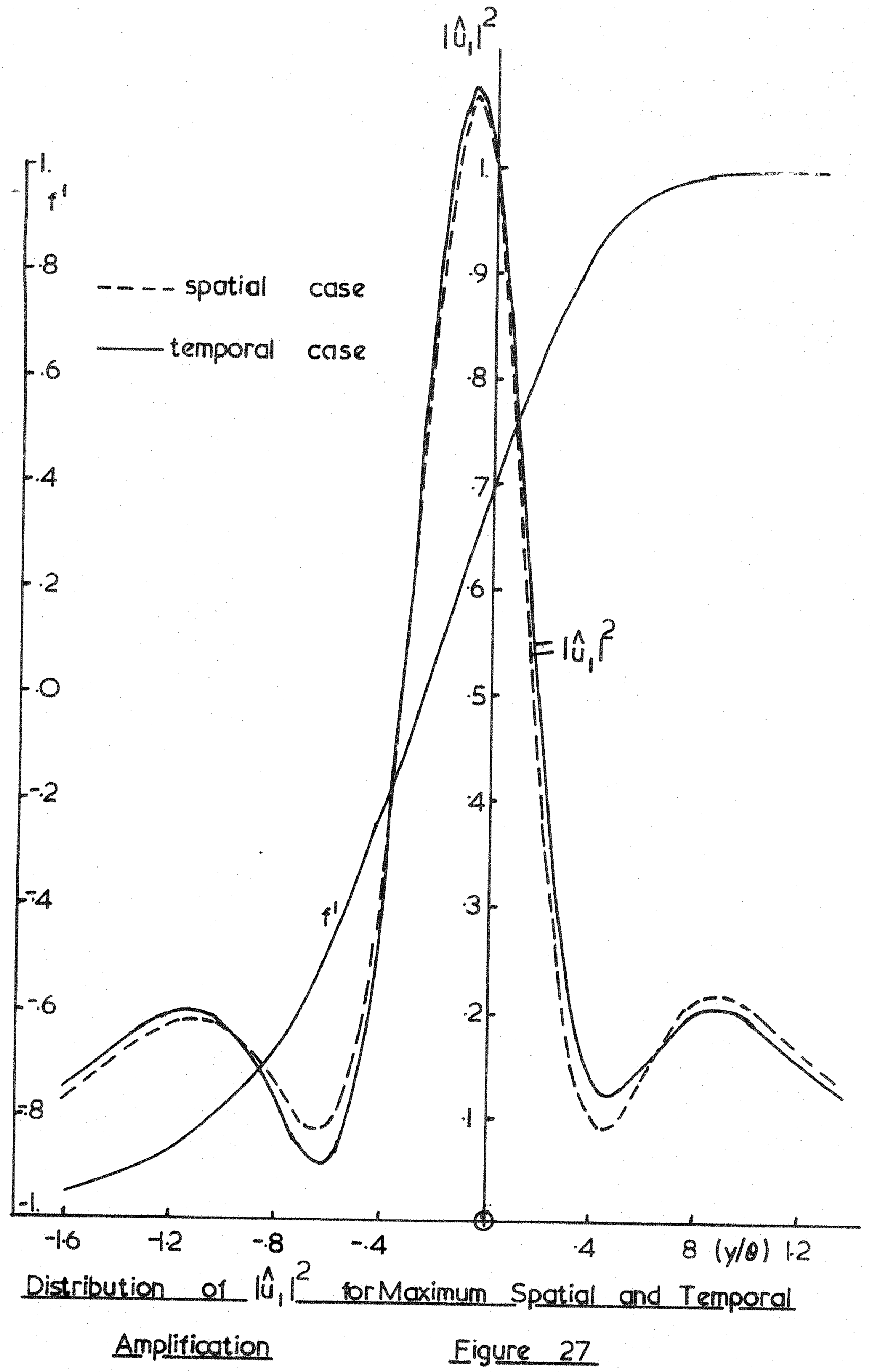




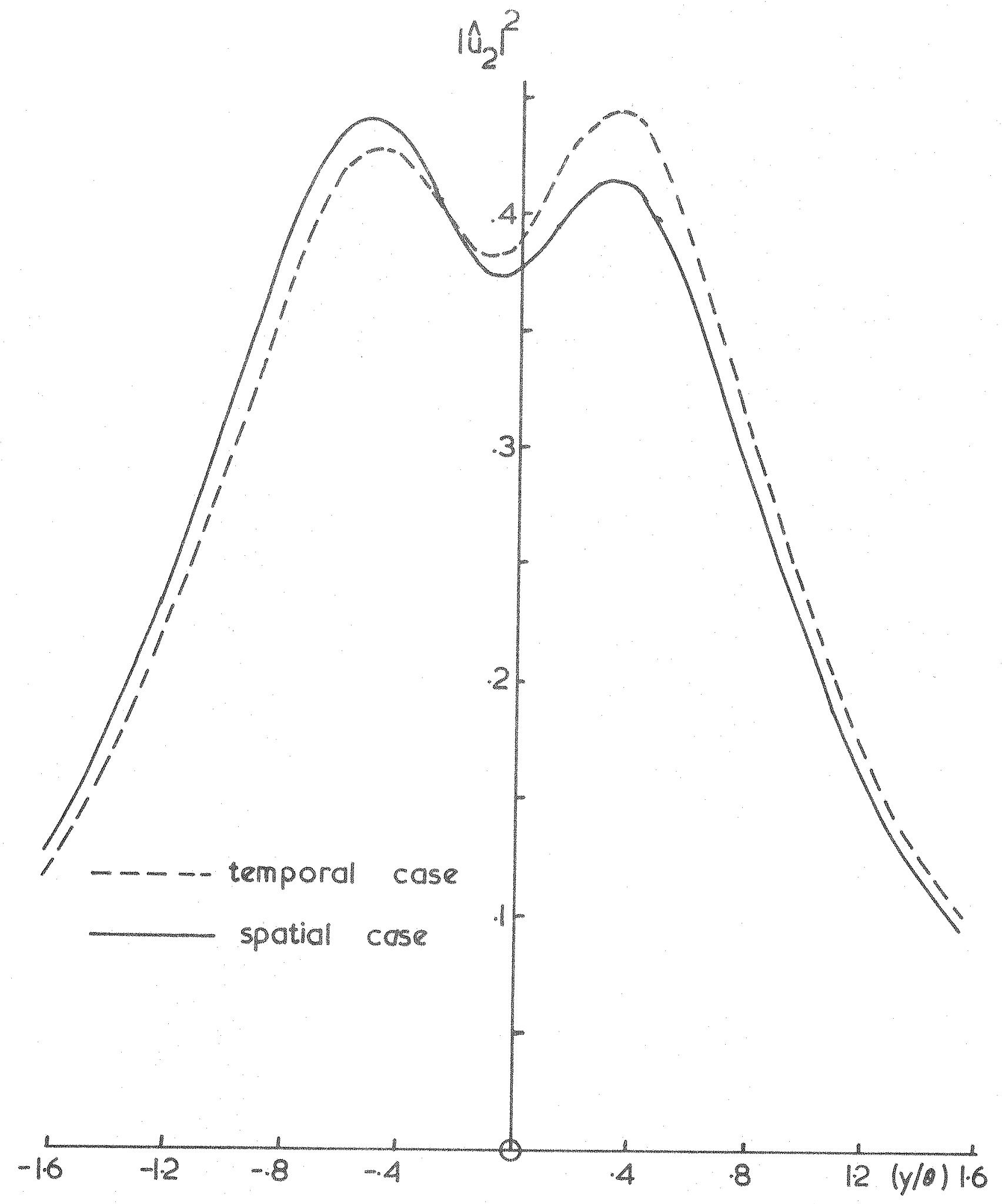

Distribution of $\left|\hat{\omega}_{2}\right|^{2}$ for Maximum Spatial and Temporal Amplification Figure 28 


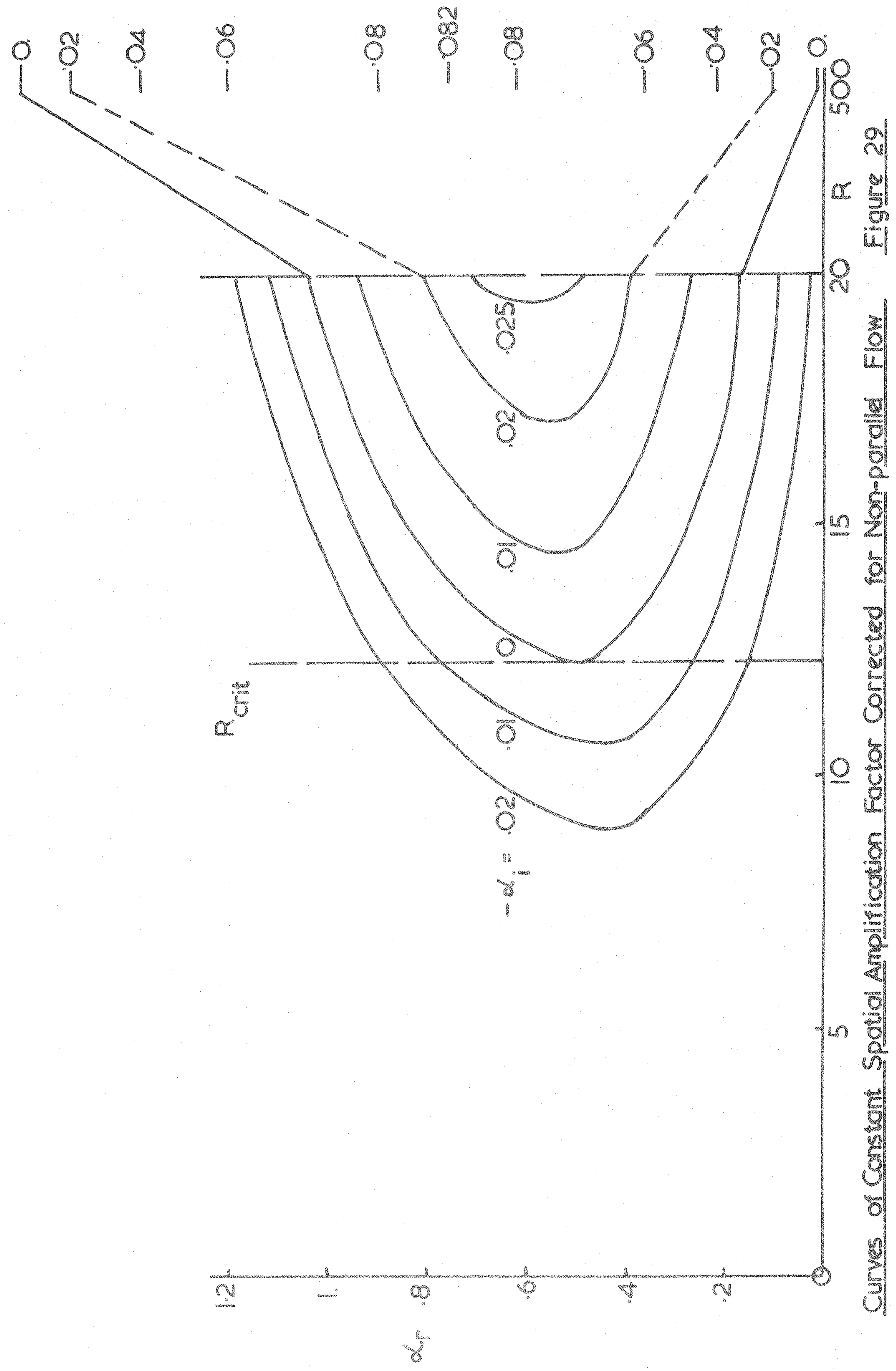




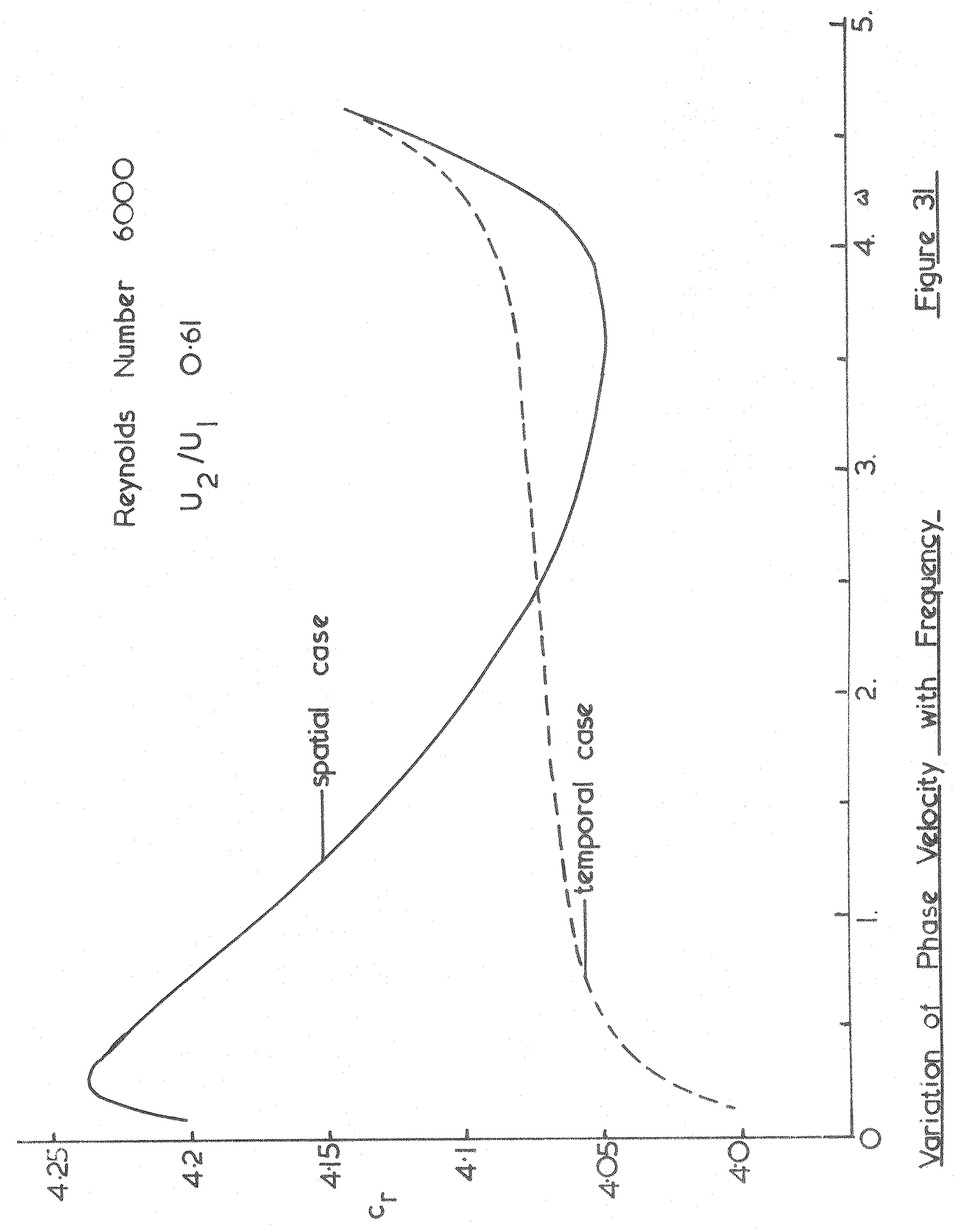




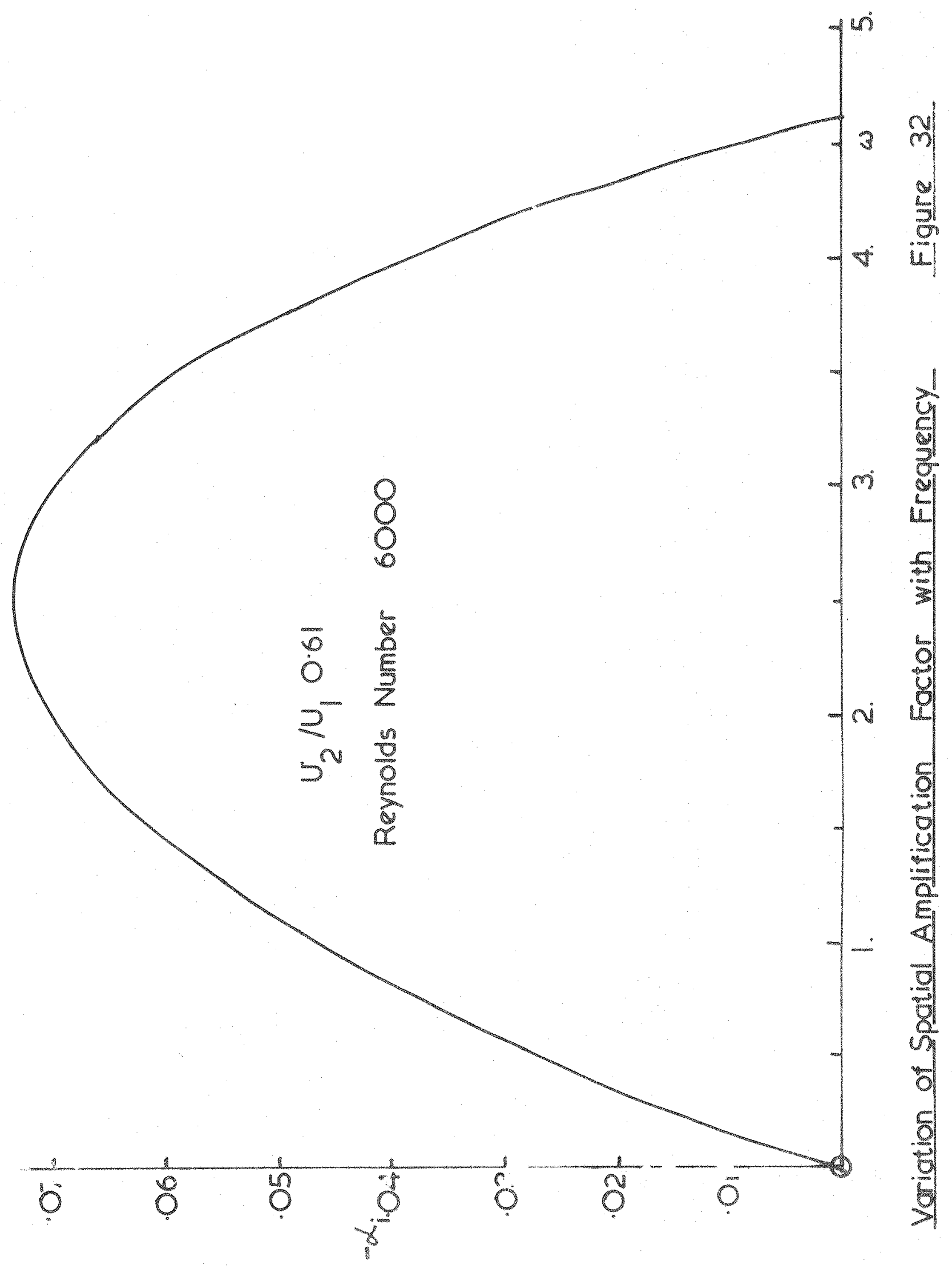




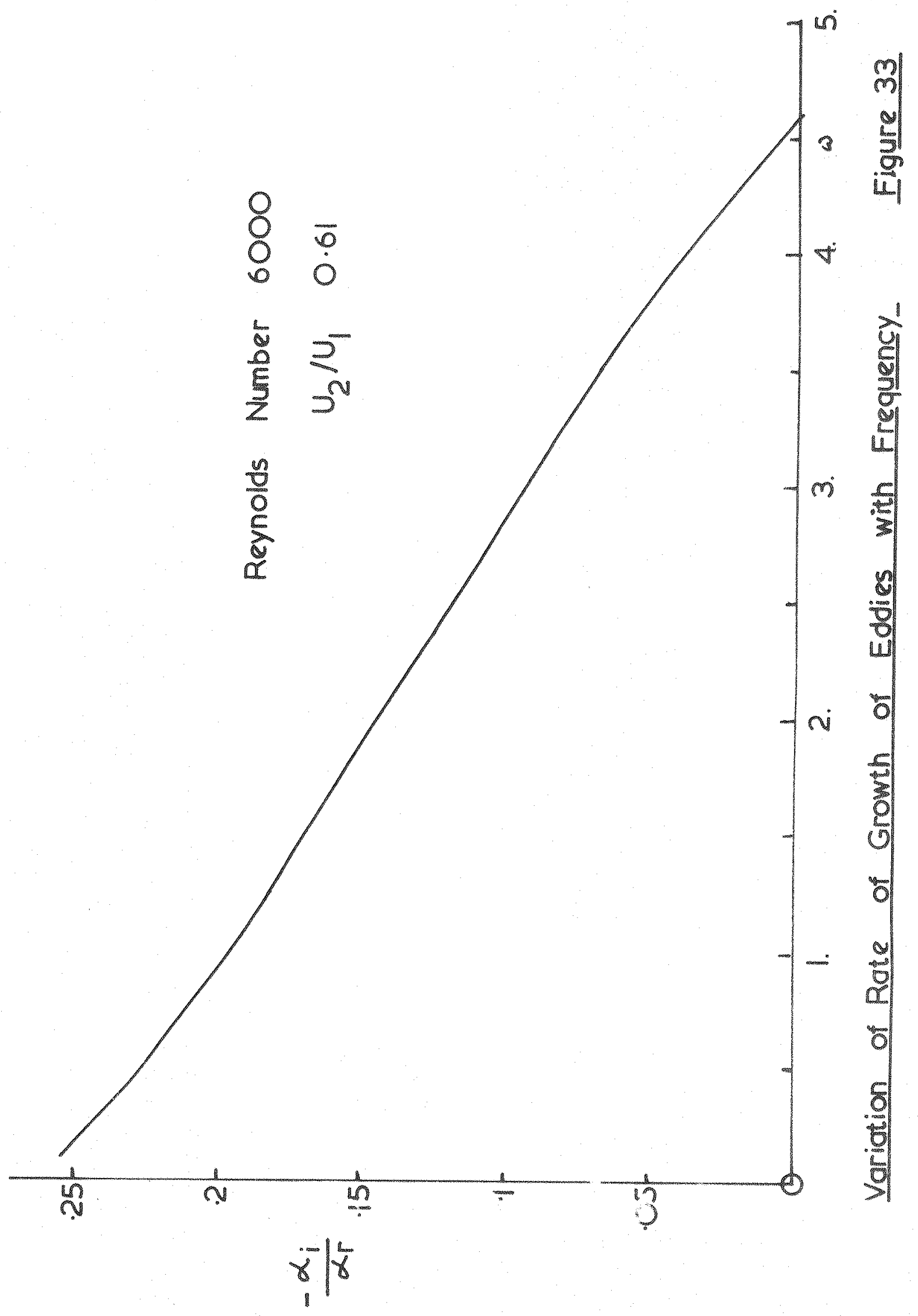




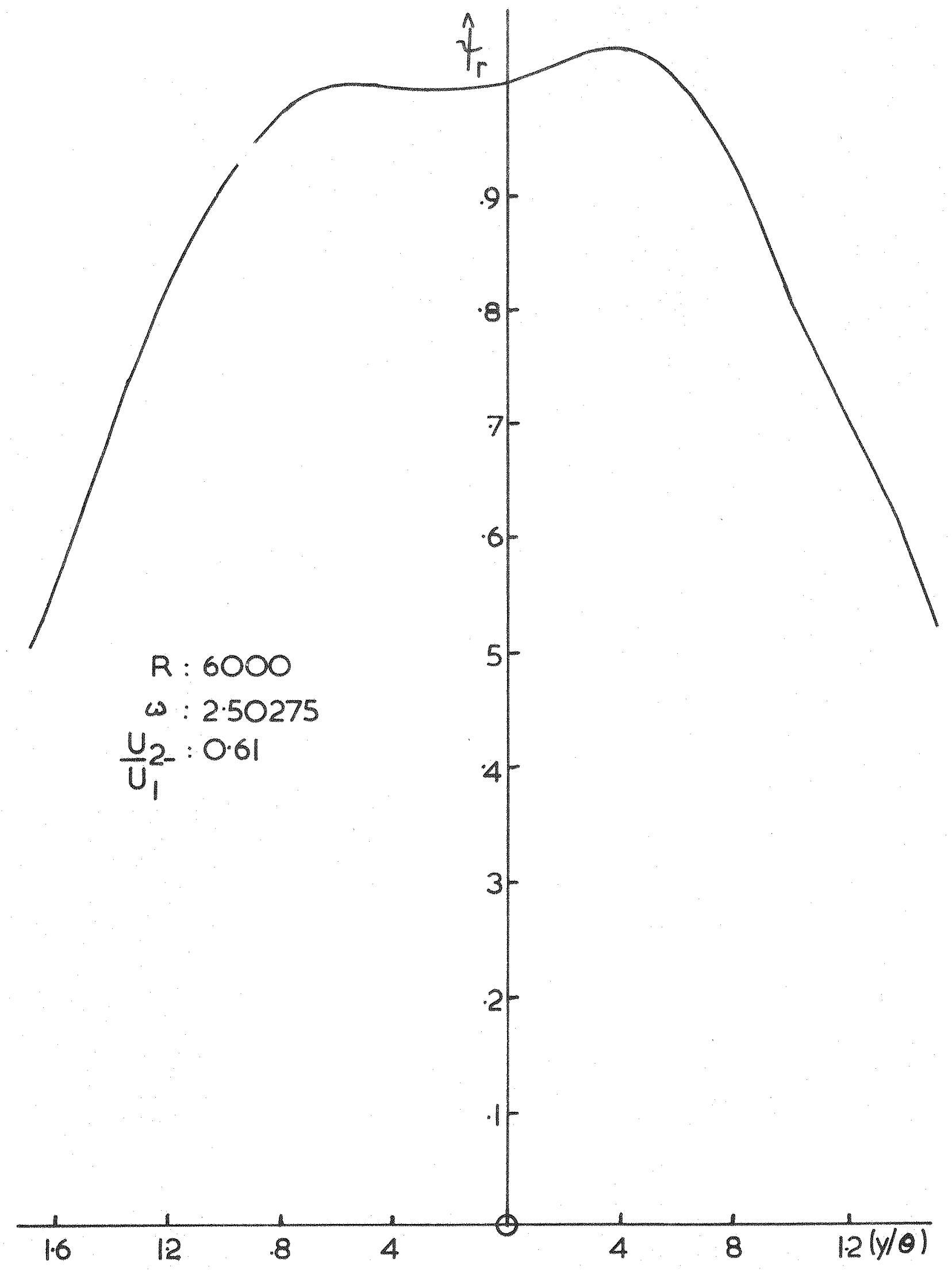

Distribution of $\mathcal{F}_{r}(y / \theta)$ for Maximum Spotial Amplification Figure $34(a)$ 

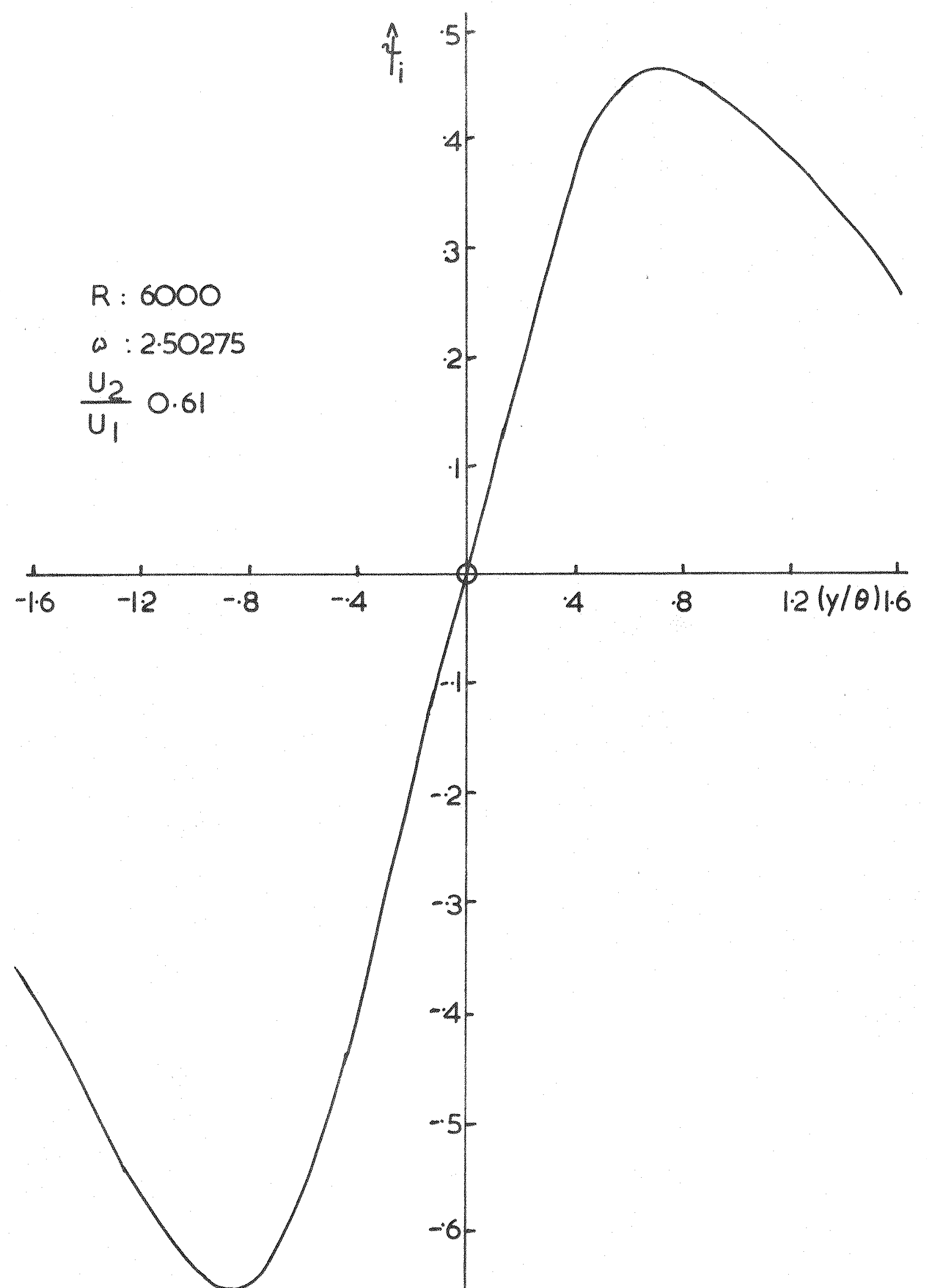

Distribution of $f_{i}(y / \theta)$ for Maximum Spatial Amplification Figure $34(b)$ 


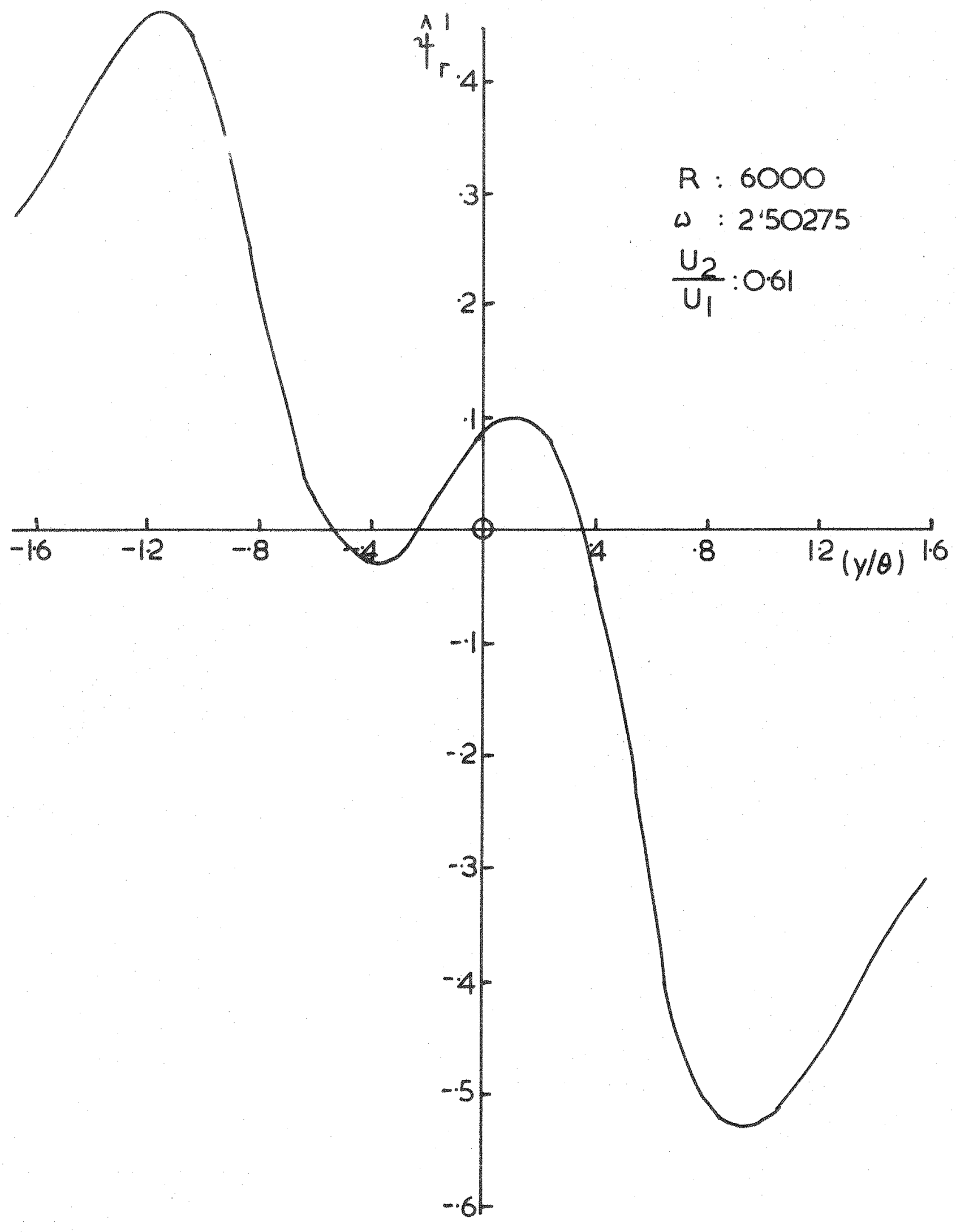

Distribution of $\hat{\psi}_{r}^{\prime}(y / \theta)$ for Maximum Spatial Amplification Figure 35(a) 


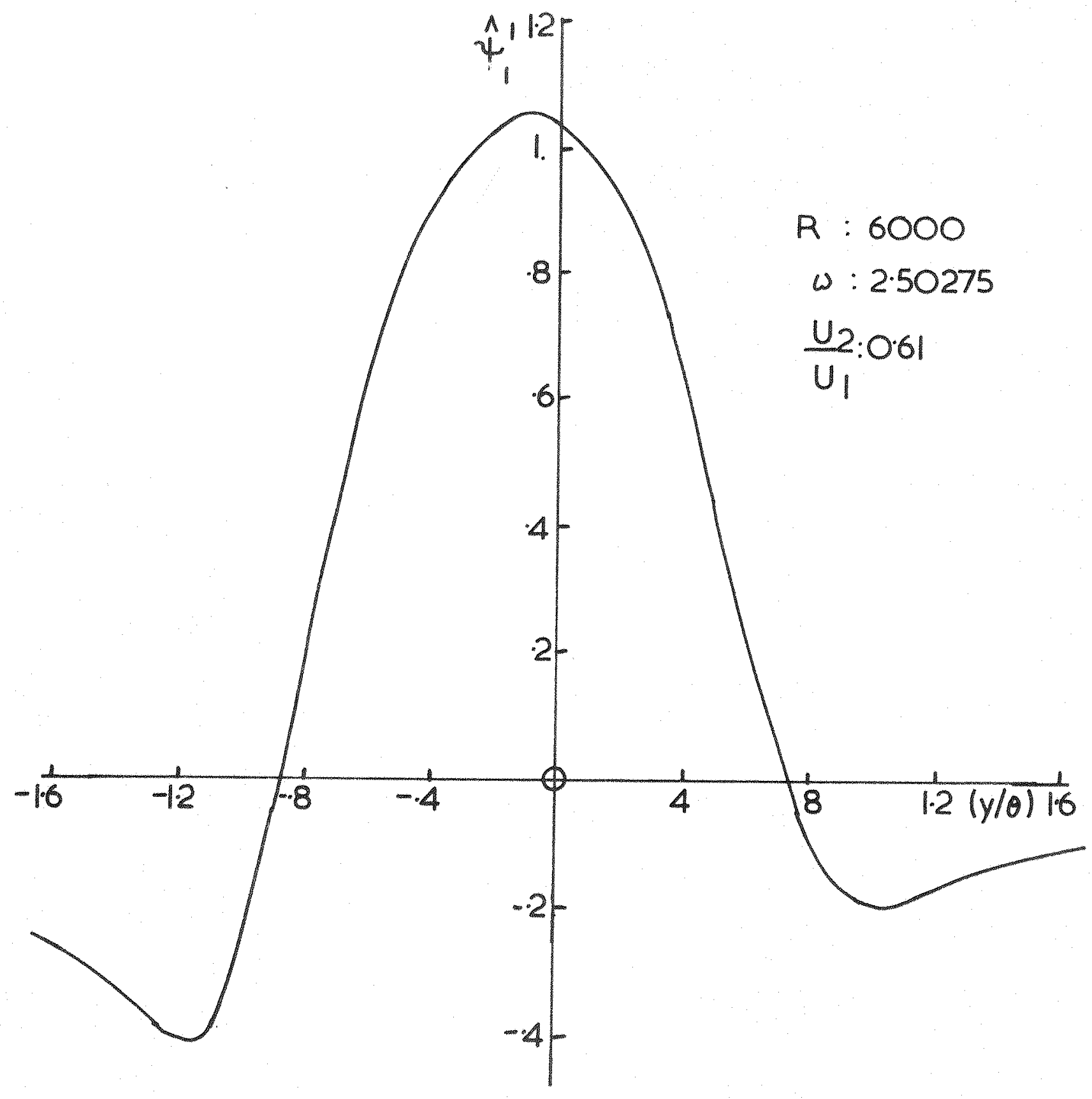

Distribution of $\hat{\psi}_{i}^{\prime}(y / \theta)$ for Maximum Spatial Amplification

Figure $35(b)$ 


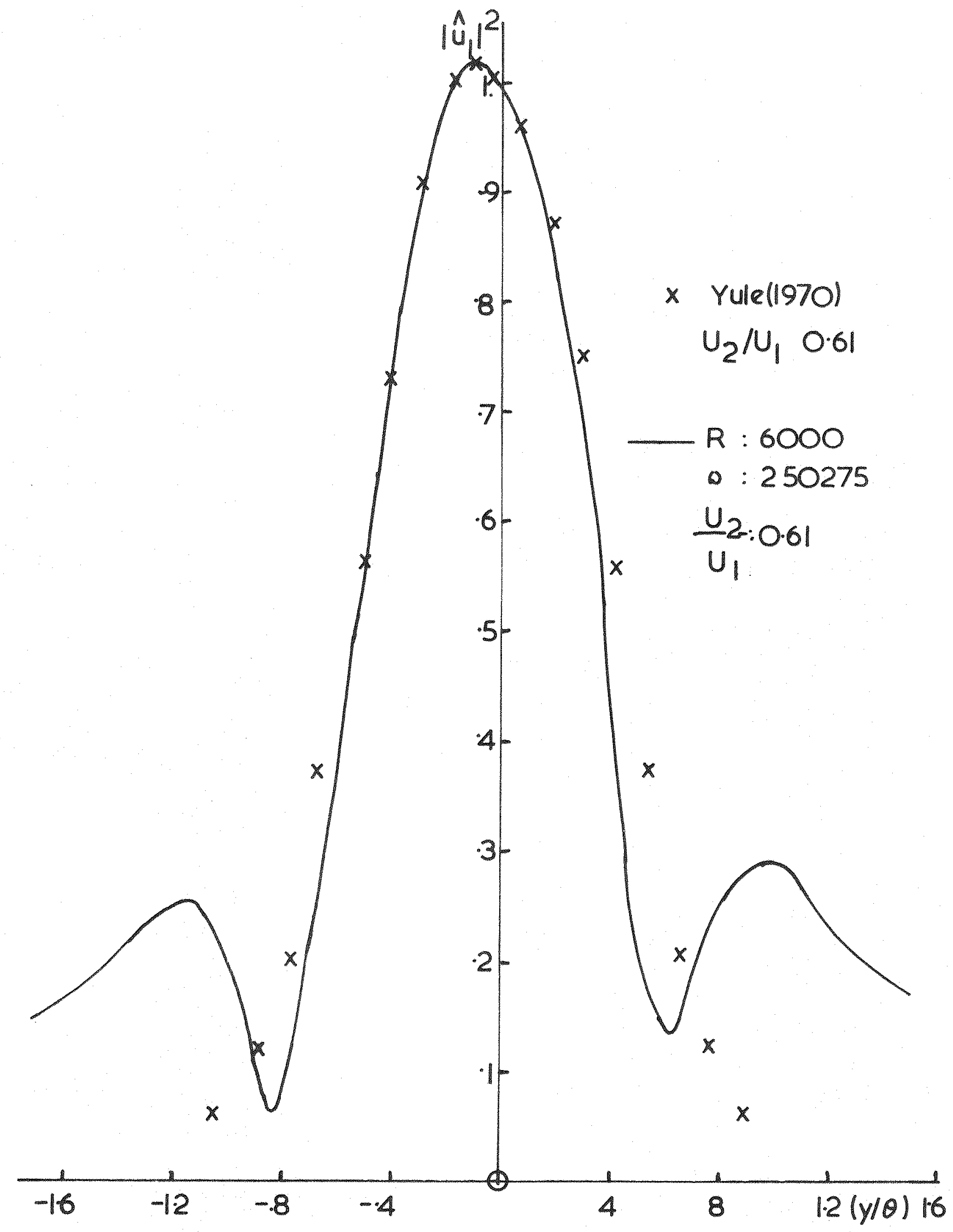

Distribution of $1 \hat{A}_{1} \mathrm{Z}^{2}$ for Maximum Spatial Amplification Figure 36 


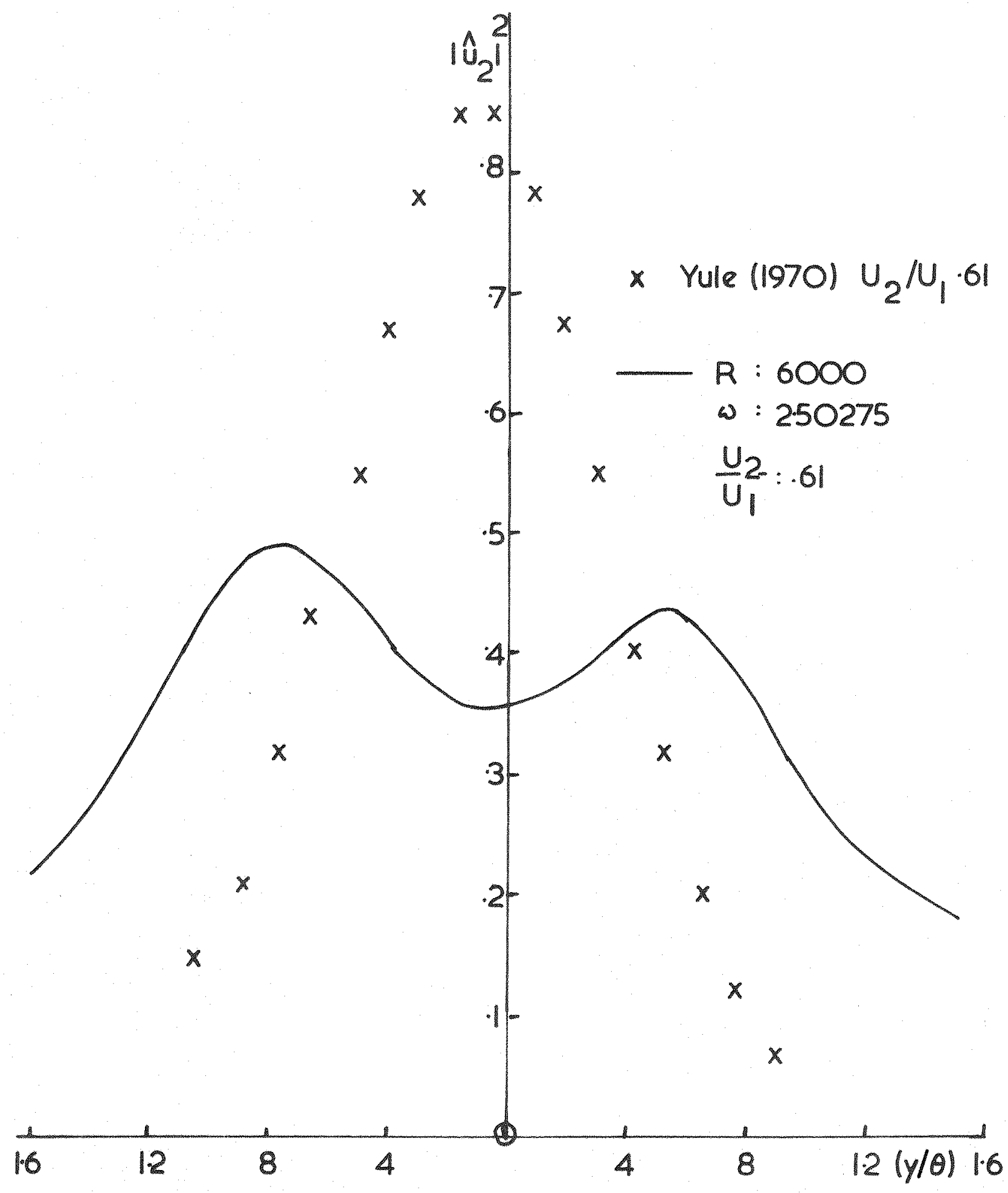

Distribution of $\mathrm{lu}_{2}{ }^{2}$ for Maximum Spatial Amplification Figure $\quad 37$ 


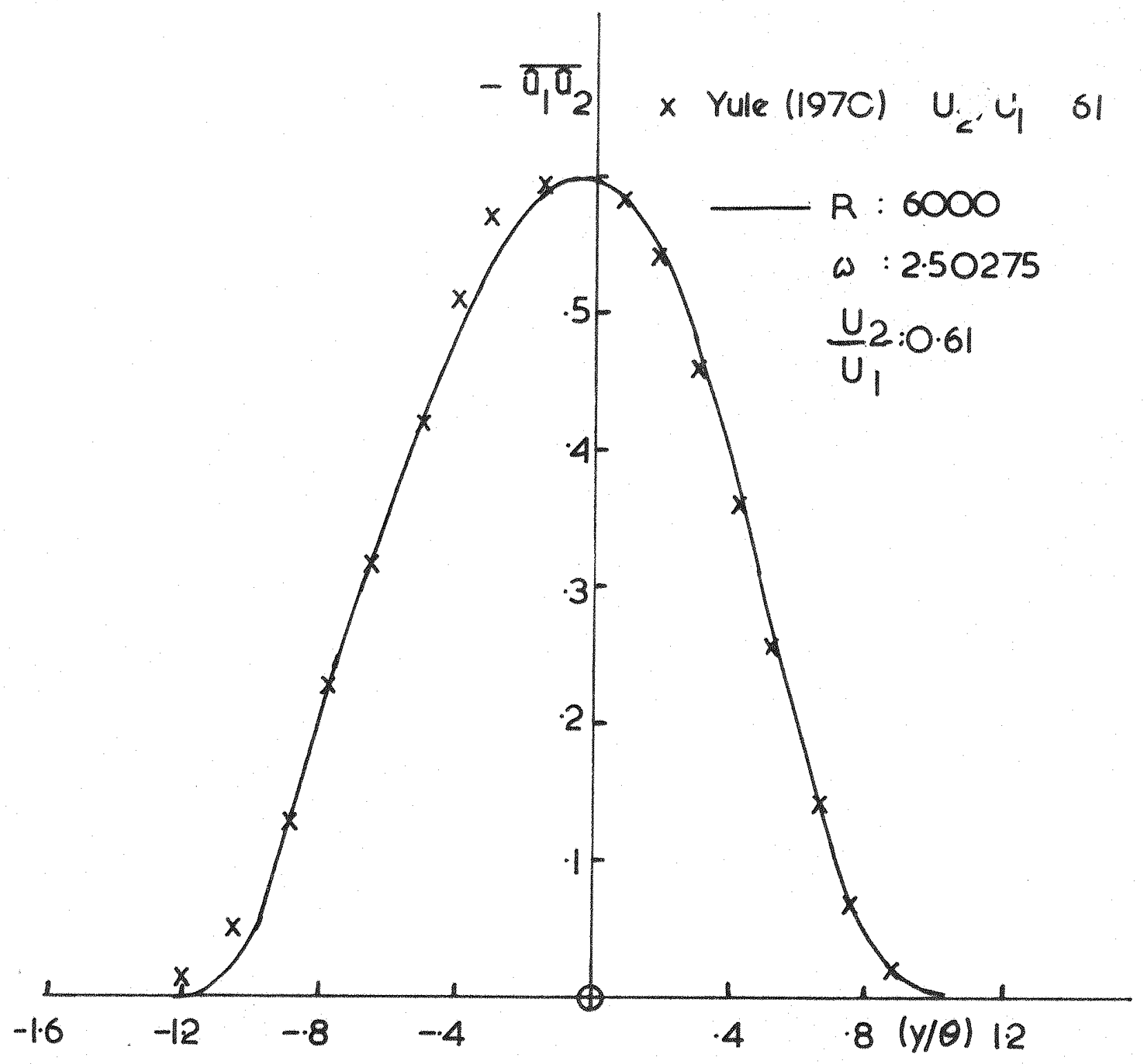

Distribution of $-\overline{0,0}$ for Maximum Spatial Amplification Figure $\quad 38$ 


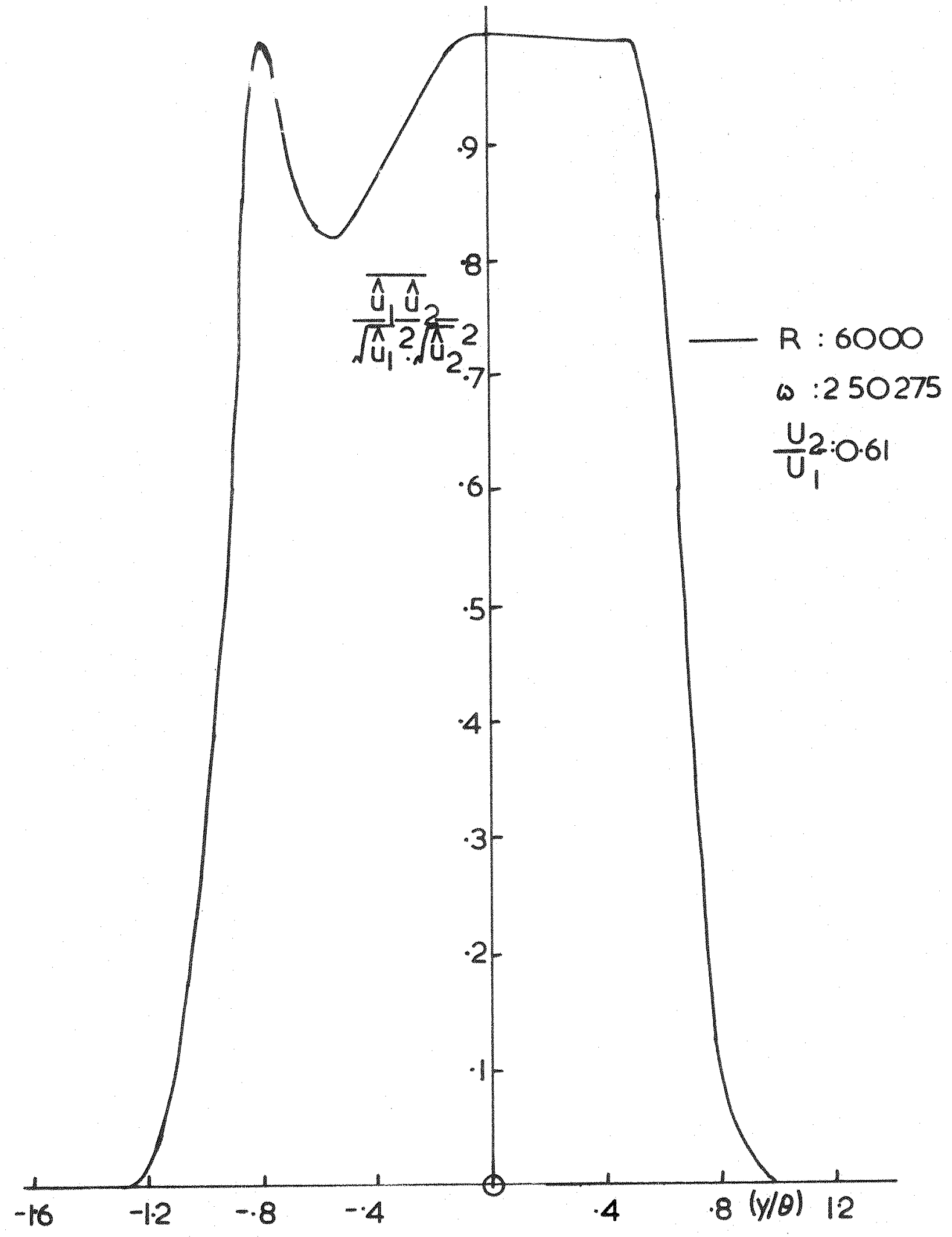

Distribution of Shear Correlation Coefficient for Maximum Spatial Amplification Figure 39 


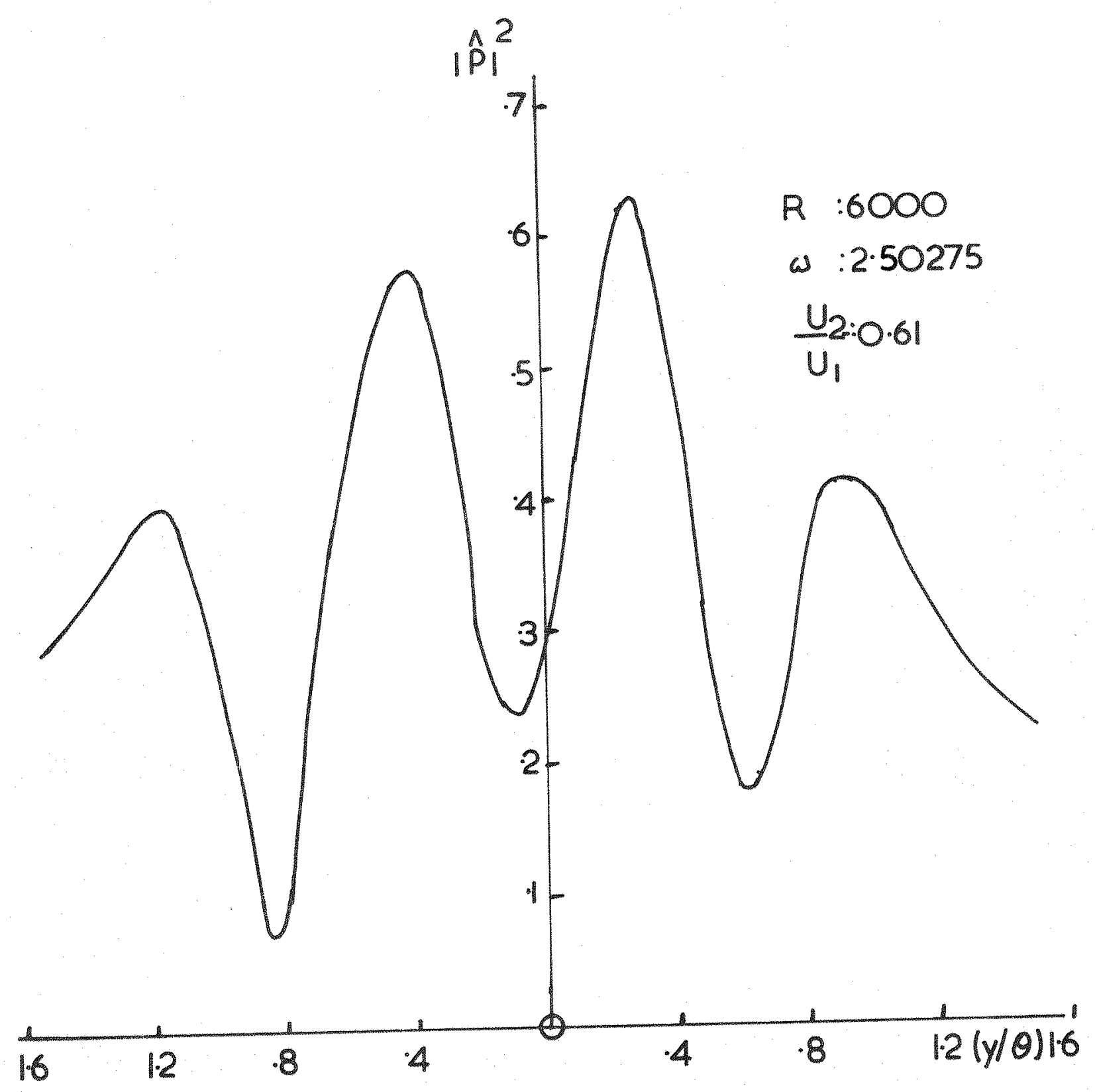

Distribution of $|\hat{p}|^{2}$ for Maximum Spatial Amplification Figure 40 


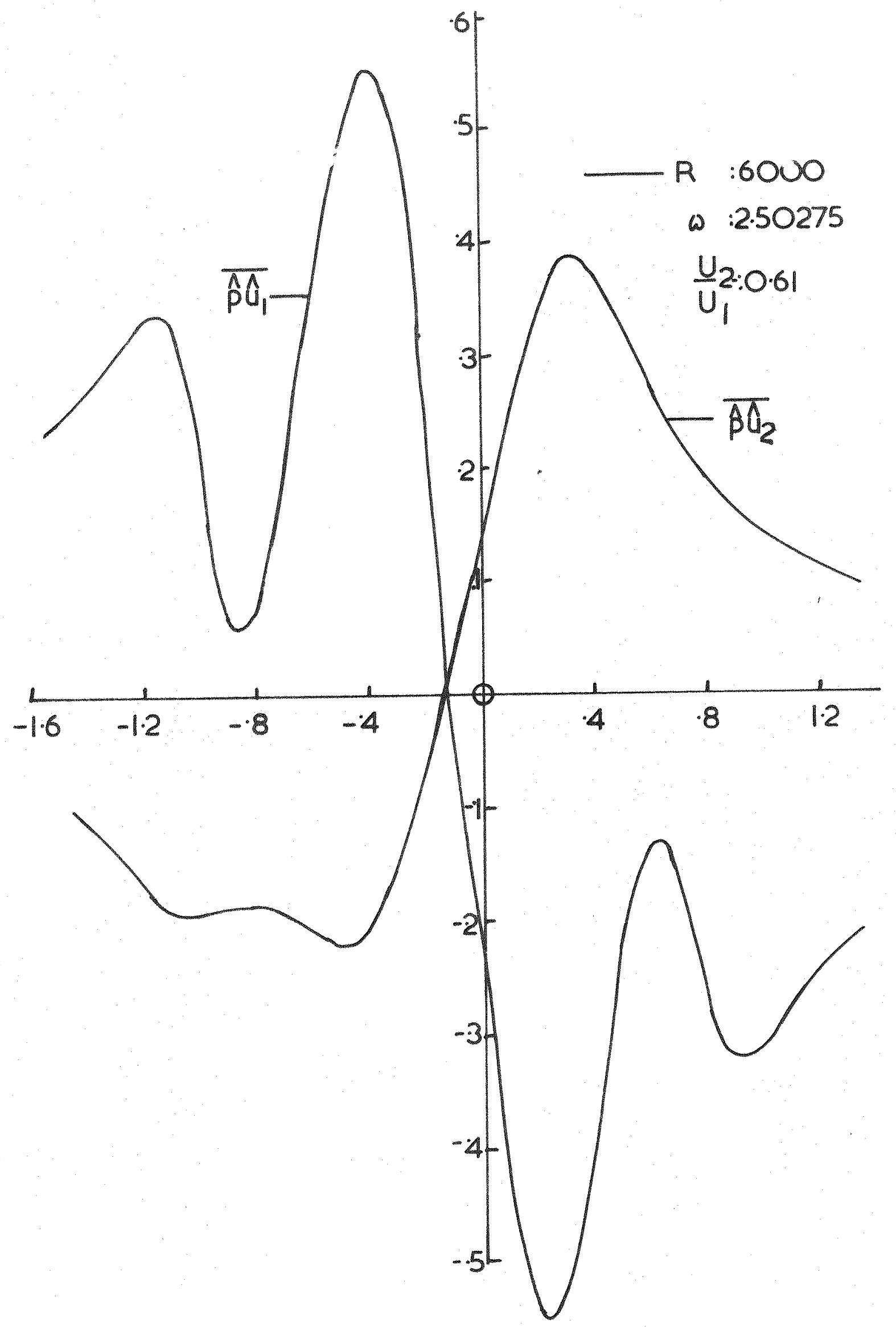

Distributions of $\overline{\mathrm{p}}_{1} \& \overline{\mathrm{pu}}_{2}$ for Maximum Spatial Amplification Figure 41 


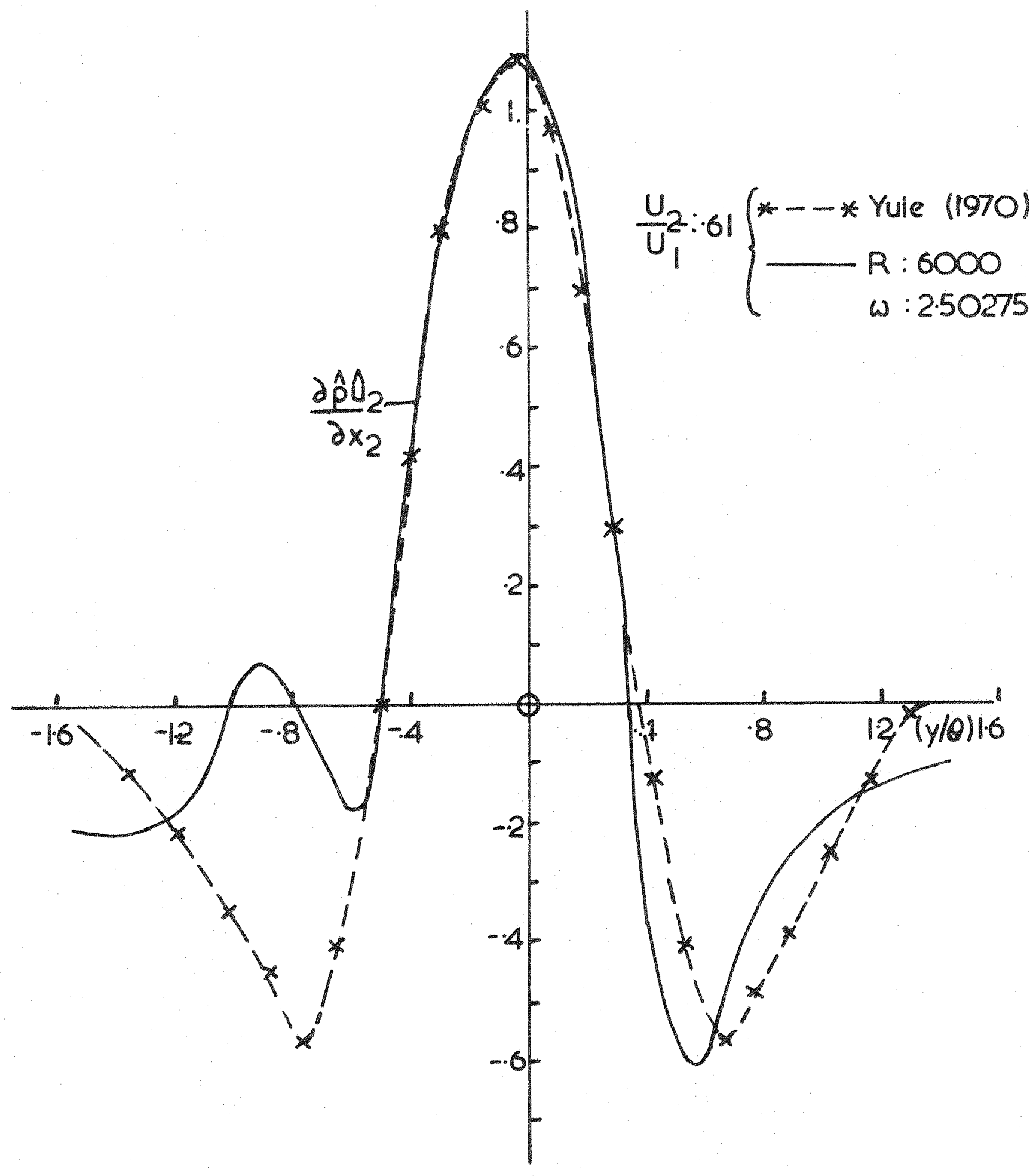

Distribution of $\frac{\partial p u_{2}}{\partial x_{2}}$ for Maximum Spatial Amplification

Figure 42 


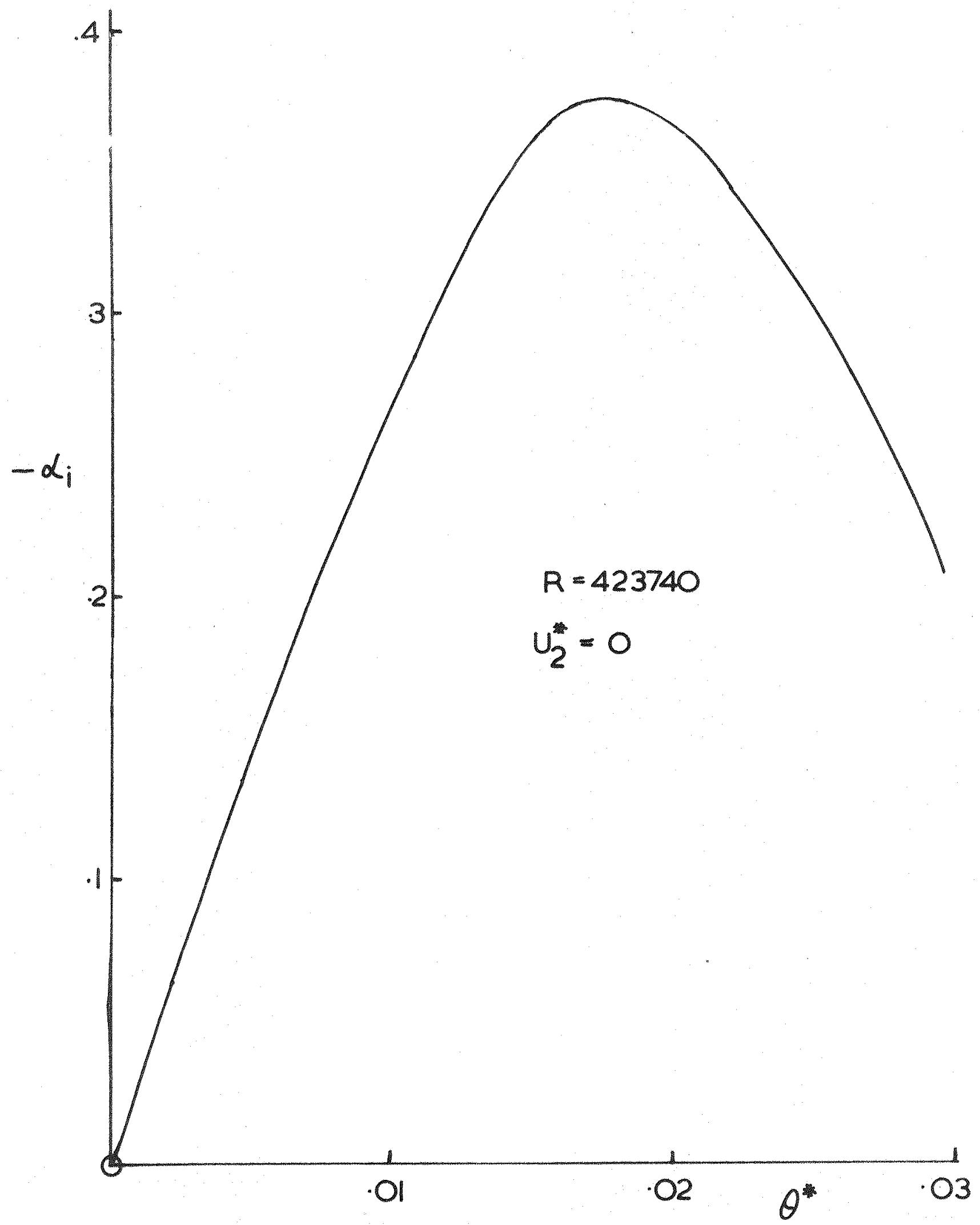

Amplification Factor ogainst $\theta^{*}$ Figure 43 


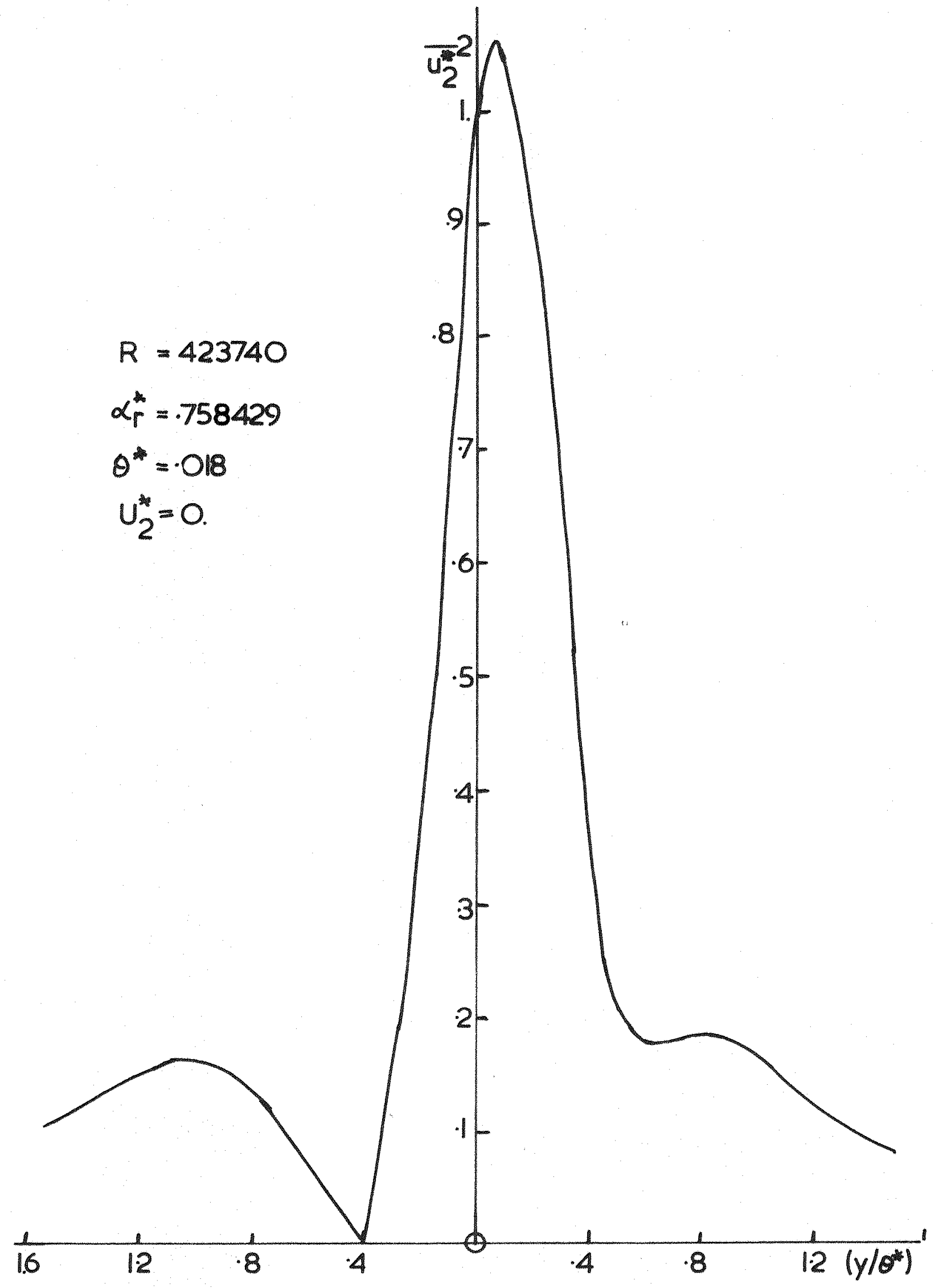

Distribution of ${\overline{u_{2}}}_{2}^{2}$ for $\theta^{*}=018$ Figure 44 


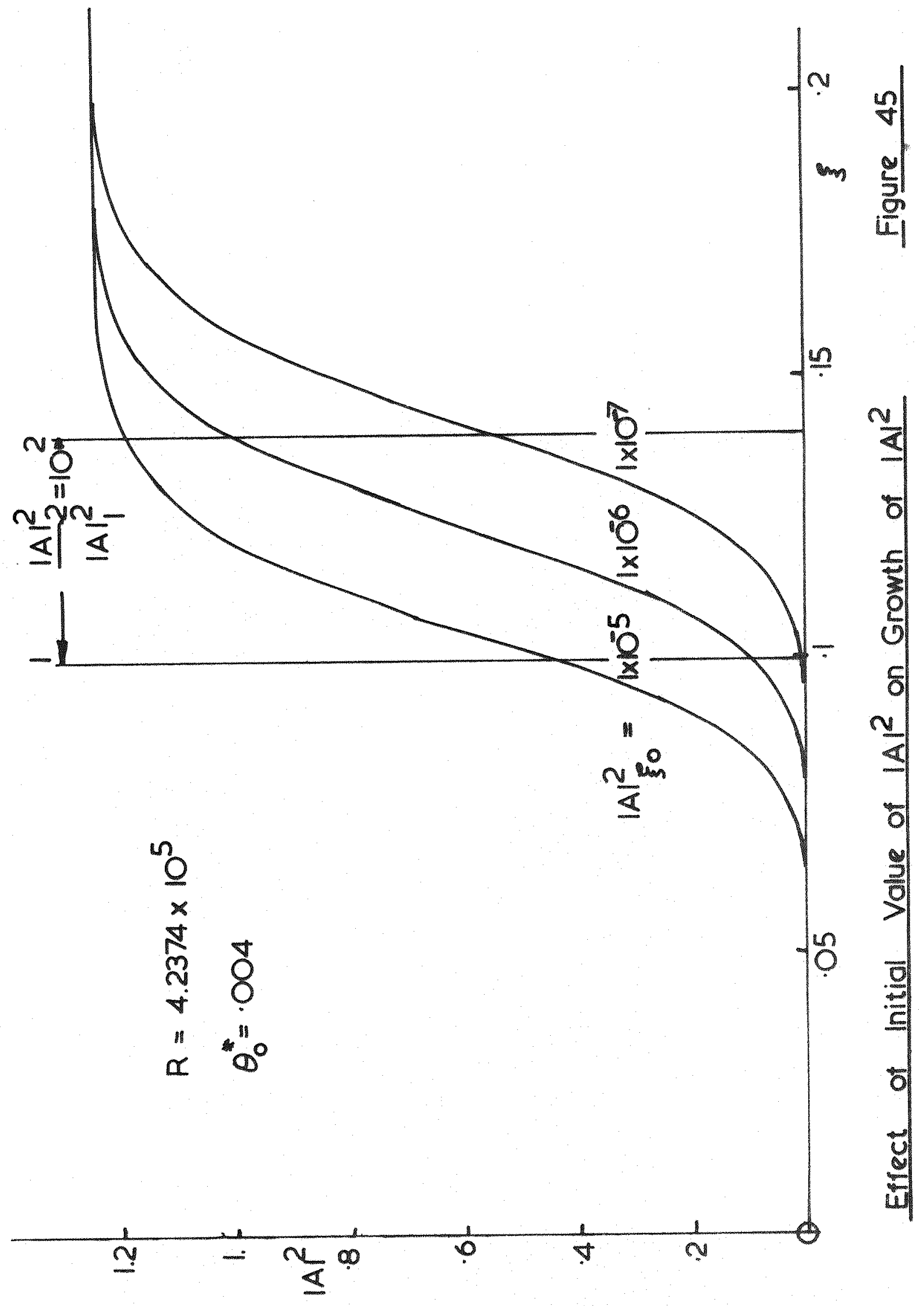




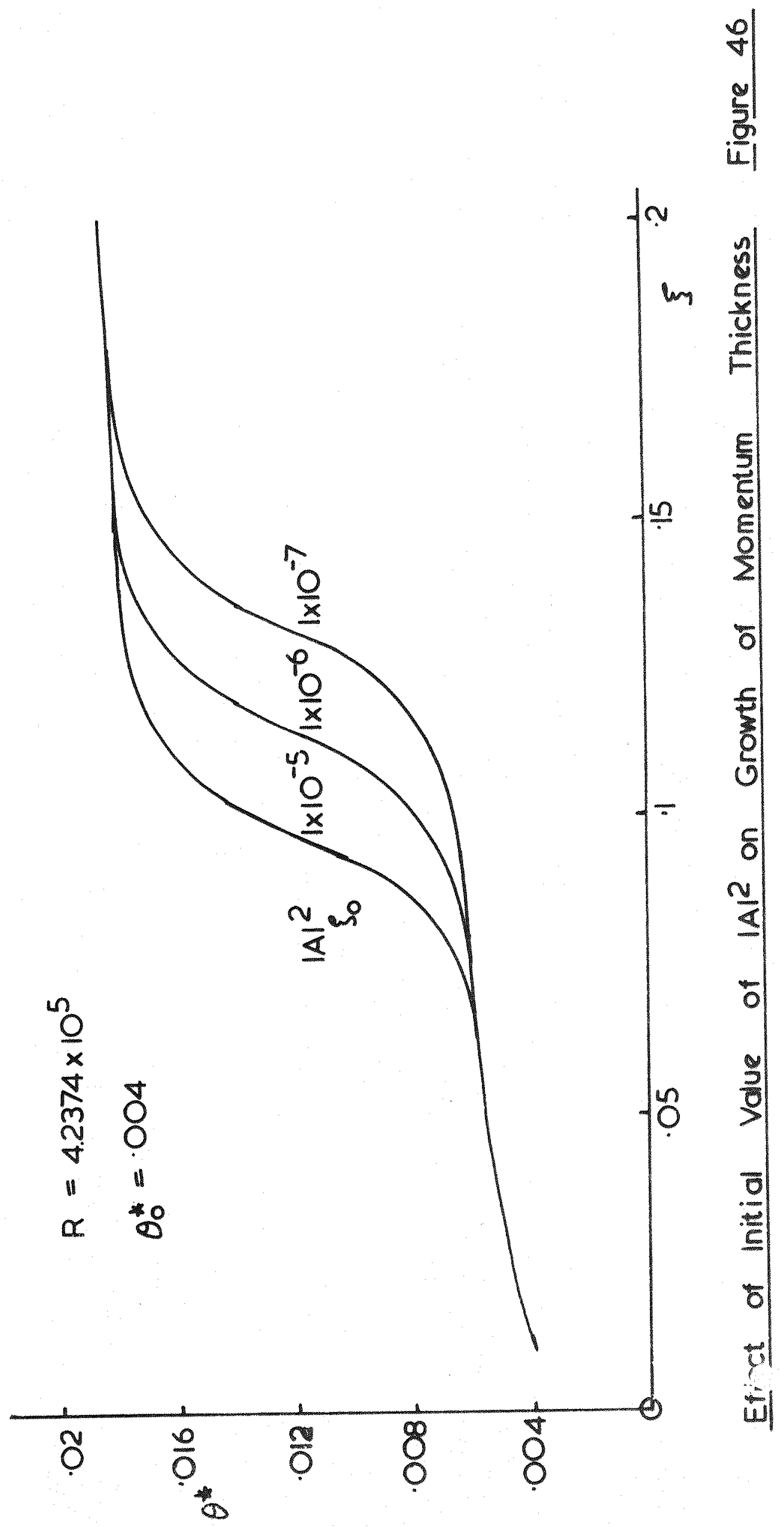




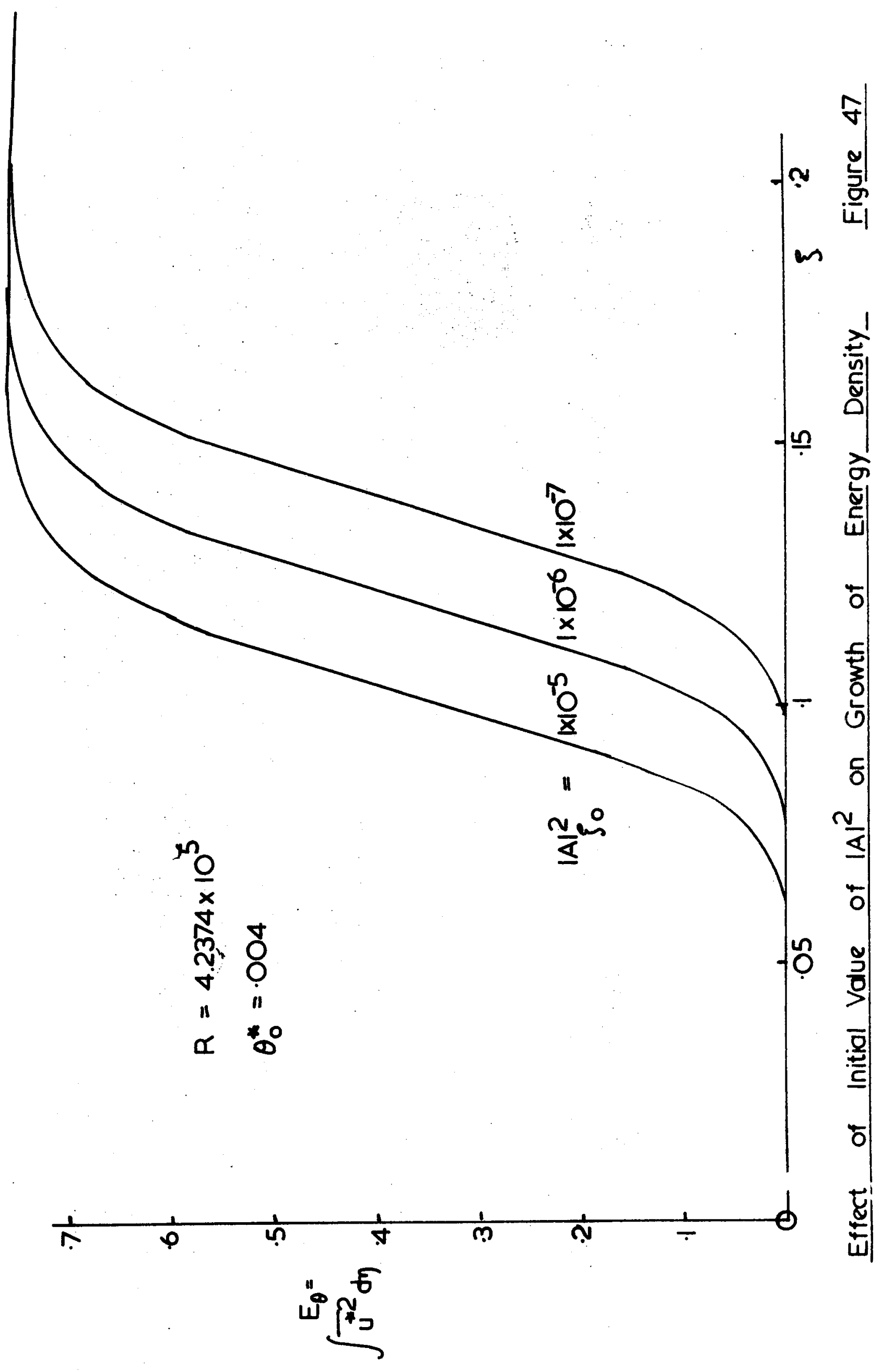




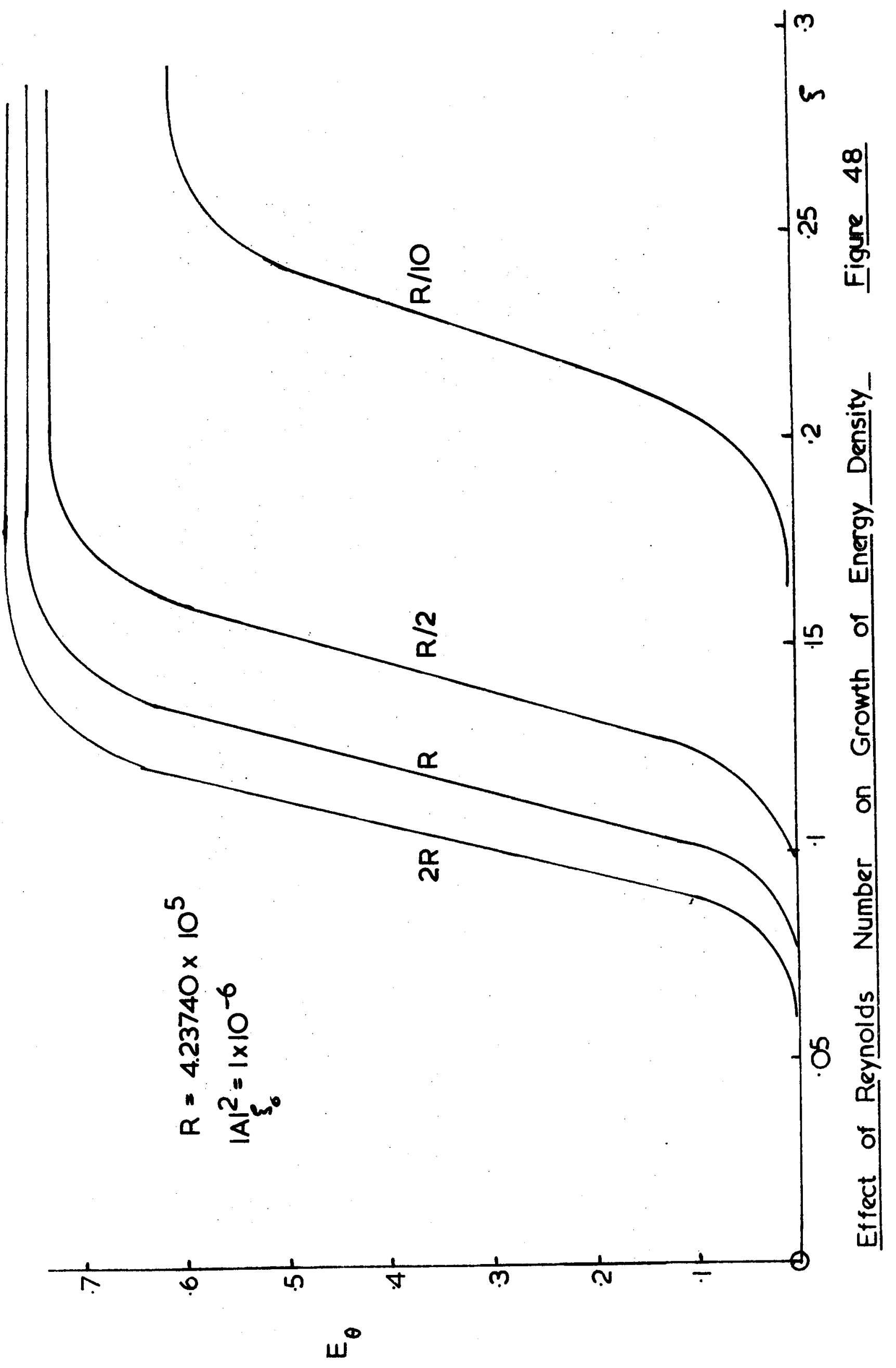




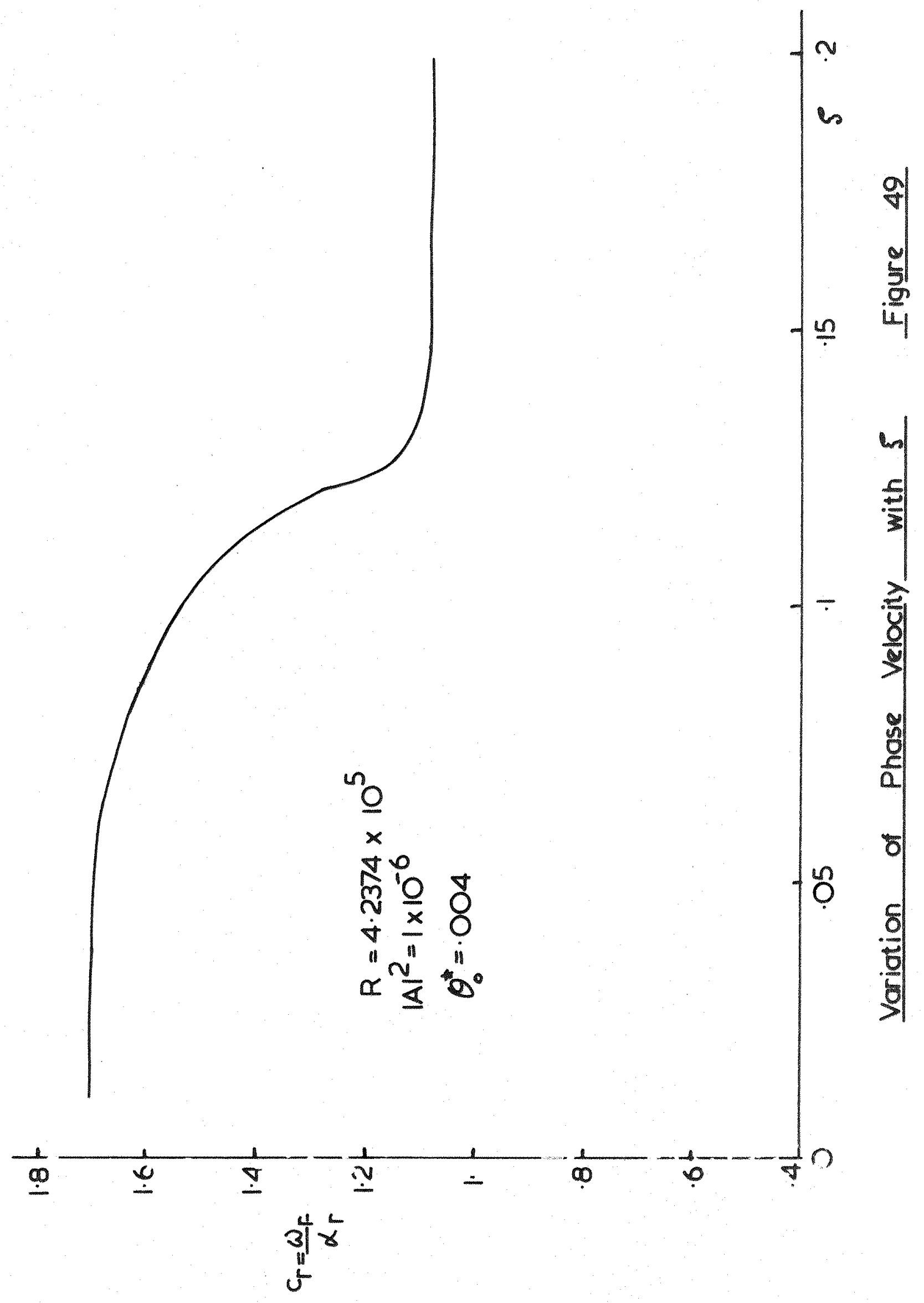




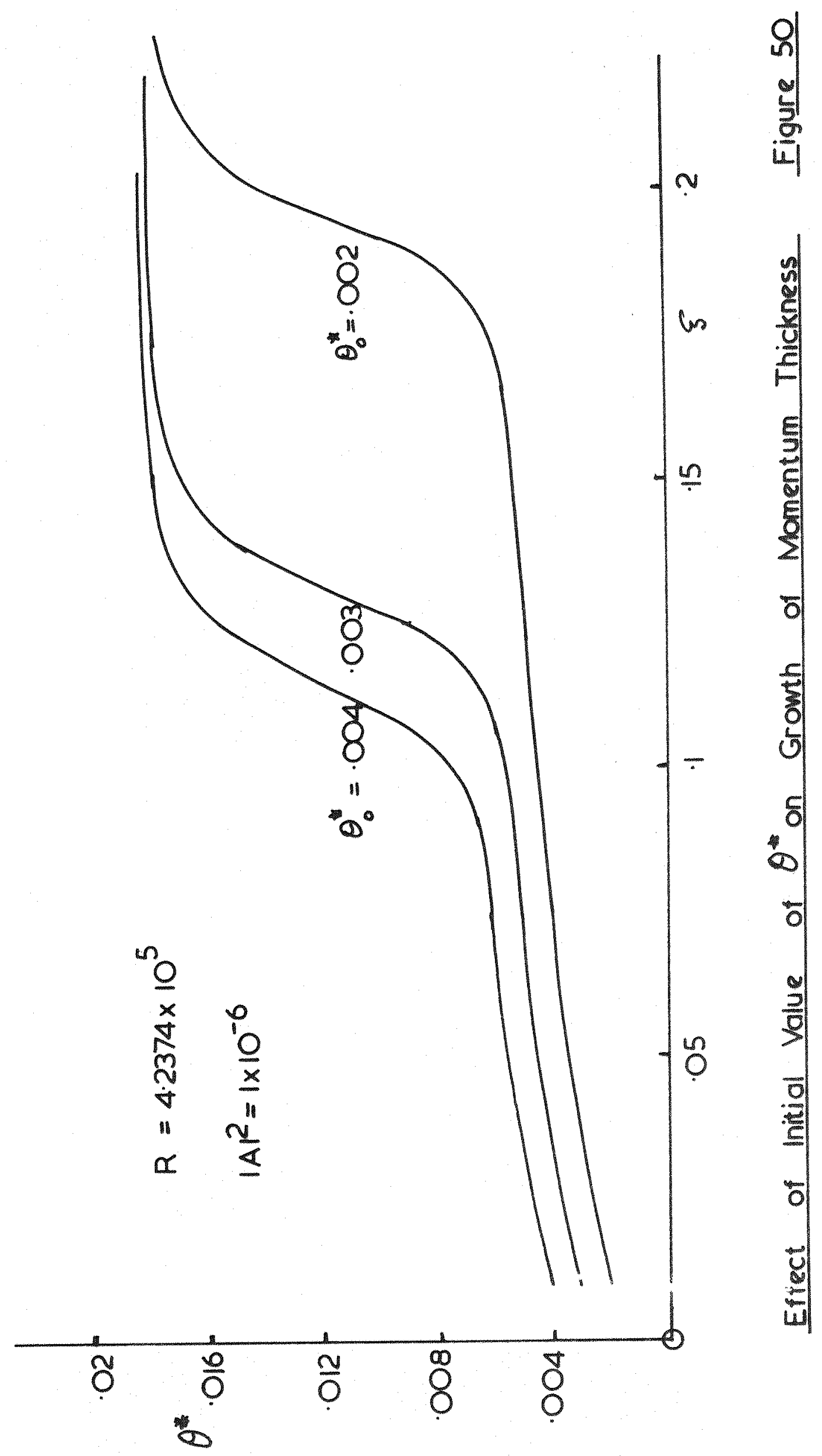

